\title{
Optocoupling in CMOS
}

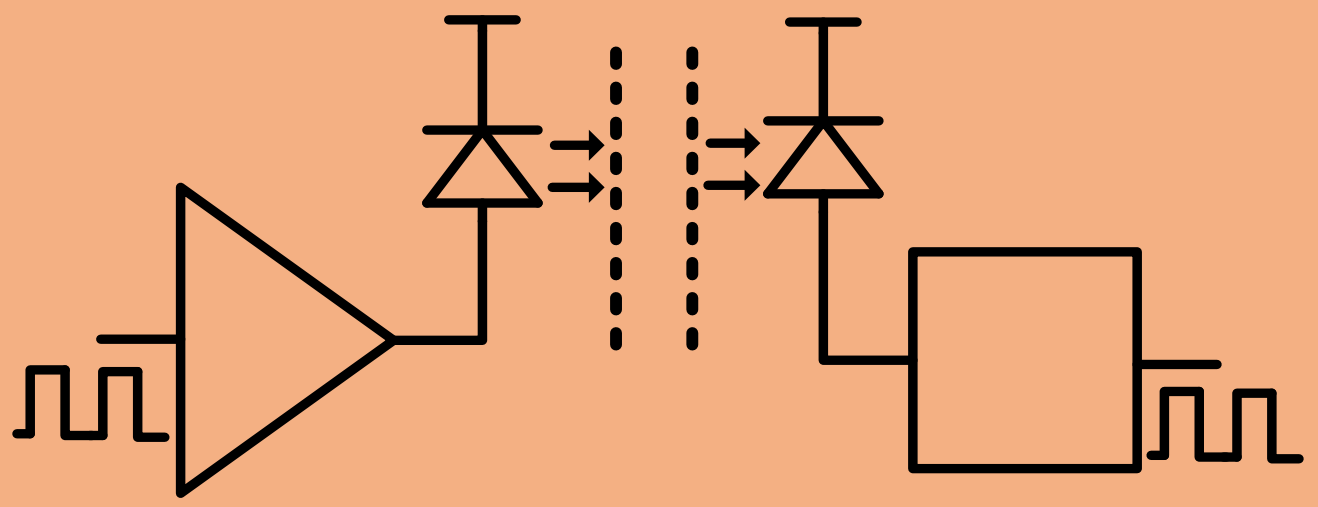

Vishal Agarwal 



\section{OPTOCOUPLING IN CMOS}





\title{
OPTOCOUPLING IN CMOS
}

\author{
DISSERTATION
}

to obtain

the degree of doctor at the University of Twente, on the authority of the rector magnificus, prof. dr. T. T. M. Palstra,

on account of the decision of the Doctorate Board, to be publicly defended

on Wednesday 16th of January 2019 at 16:45 hours

by

Vishal Agarwal

born on the 28th of December, 1988

in Chaibasa, India 
Samenstelling promotiecommissie:

Voorzitter en secretaris:

prof. dr. J.N. Kok

University of Twente, EWI

Promotor:

prof. dr. ir. B. Nauta

University of Twente, EWI

Co-promotor:

dr. ir. A.J. Annema

University of Twente, EWI

Leden:

dr. ir. R.J.E. Hueting

University of Twente, EWI

prof. dr. J. Schmitz

University of Twente, EWI

prof. L. W. Snyman

University of South Africa, Johannesburg

prof. E. Charbon

prof. dr. L.K. Nanver

École Polytechnique Fédérale de Lausanne, Switzerland

Delft University of Technology, The Netherlands

This work is part of the Optocoupling in CMOS project

$\widehat{\mathrm{N} \mathcal{W}} \mid$\begin{tabular}{|l} 
Applied and \\
Engineering Sciences
\end{tabular} (no. 12835) and is supported financially by the Applied and Engineering Science division (TTW) of the Netherlands Organization for Scientific Research (NWO).

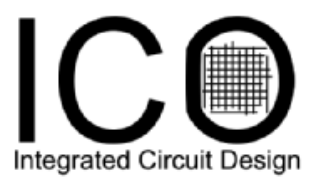

Integrated Circuit Design, University of Twente, $7500 \mathrm{AE}$ Enschede, the Netherlands.

Typeset with LATEX.

Copyright (C) 2019 by Vishal Agarwal, Enschede, The Netherlands. All rights reserved. Back Cover photo: from wikipedia (under free to use license).

ISBN $\quad 978-90-365-4707-9$

DOI $\quad 10.3990 / 1.9789036547079$

http:/ /dx.doi.org/10.3990/1.9789036547079 
This dissertation has been approved by:

Promotor: prof. dr. ir. B. Nauta

Co-promotor: dr. ir. A. J. Annema 



\section{ABStract}

Around 1970, a new class of circuits that monolithically integrated high power devices with control circuits were proposed. These new circuits, referred to as "power integrated circuits" (PICs) enabled low cost implementation of high power systems such as voltage regulators and high-power amplifiers. Since then, PICs have evolved and are currently used in many systems like audio amplifiers, motor controllers and automotive electronics. The revenue generated by such systems has been estimated to be more than 10 billion USD in 2016.

While according to the "Moore's law", continuous advancements in traditional silicon ( $\mathrm{Si}$ ) complementary metal-oxide-semiconductor (CMOS) technologies have been done to implement more processing capabilities at a lower cost, special CMOS technologies have also been developed for PICs to enable some of the "More-than-Moore" applications. These special Bipolar-CMOS-DMOS (BCD) technologies, currently known as the "smart power" integrated circuit technologies, combine the "smartness" of digital circuits with the "power" of power devices.

In many systems in which smart power technologies have been implemented, for safety and/or avoiding interference data communication between different voltages domains with galvanic isolation is required. Communication by means of light across different voltage domains is attractive for such isolated communication links. This is because light eliminates any direct conductive path between the isolated circuits and is robust to external electrical and magnetic interferences.

The principle of data communication with light across isolated voltage domains is used in so-called "optocouplers". In optocouplers, light emitted by an emitter in one voltage domain is detected by a receiver in an another voltage domain. At present, only discrete optocouplers are available; however a discrete implementation increases the cost for PICs. Monolithic implementation of optocouplers without any additional processing (standard CMOS) would be a disruptive technology, enabling several new "smart" PICs at lower cost and area requirements. Research on enabling these integrated optocouplers has been the focus of this research. Most important performance metrics of these integrated optocouplers are the area, energy-per-bit, data rate and "amount" of isolation between two voltage domains.

The main issue with the monolithic implementation of optocouplers 
is the absence of an efficient light source in CMOS technologies. Being an indirect band gap semiconductor, forward biased Si light-emitting diodes (LEDs) emit light at infrared wavelengths with low efficiency, while Si photodetectors (PDs) have a relatively low responsivity at those wavelengths. However, Si avalanche mode LEDs (AMLEDs) have a broad emission spectrum in the visible range which has a significant overlap with the responsivity of Si PDs. Therefore in this thesis, the use of AMLEDs is proposed for the monolithic implementation of optocouplers. Another issue however is that $\mathrm{Si}$ AMLEDs have a relatively low electrical to optical efficiency, also referred to as quantum efficiency. To compensate for such a low quantum efficiency, single-photon avalanche diodes (SPADs) in CMOS technologies are proposed for the light detection side. As the name suggests, SPADs are highly sensitive PDs which can (theoretically) detect even a single photon.

In the first part of this thesis, the physics of avalanche diodes is discussed in detail which is important to understand the performance of AMLEDs and SPADs. It is shown that the avalanche phenomena can be described by random telegraph signal (RTS) phenomena in the diode currents. Further, from the analysis of these RTS phenomena, the current-voltage $(I-V)$ characteristics in avalanche is explained and an accurate definition of experimental breakdown voltage is provided. Currently quenchingand-recharge circuits (QRCs) for SPADs are designed using some rules-ofthumb. The analysis of RTS phenomena can help in designing an accurate design of QRCs. The optimized QRCs can improve the speed of SPADs by a factor of 3 to 6 compared to conventional counterparts. The analysis of RTS phenomena is also important for obtaining high speed AMLEDs. It is demonstrated that diodes with more defects and/or higher leakage current are better for high speed AMLEDs, exactly the opposite requirements for SPADs.

In the second part of thesis, AMLEDs with integrated driver circuits were designed in a $140 \mathrm{~nm}$ SOI CMOS technology. A low power LED driver circuit was demonstrated which is robust to many variations in the properties of the AMLEDs and the driver circuit operating conditions. The demonstrated integrated optical transmitter can be used to achieve a low energy-per-bit for the proposed optical links. Finally, for the first time, this thesis demonstrates a monolithic optical link with very low area requirements $\left(<0.01 \mathrm{~mm}^{2}\right)$ in a standard CMOS technology. The data rates of a few Mbps at the energy consumption of a few $\mathrm{nJ} /$ bit are demonstrated.

Overall this thesis demonstrates the physics and applications of avalanche diodes for optocoupling applications. Various physics related issues of avalanche diodes are also discussed which are important for the design of AMLEDs, SPADs and the associated circuits. The results are promising and the dream of monolithic optocouplers is now closer to reality! 


\section{SAMENVATTING}

Rond 1970 werd een nieuwe klasse elektronische schakelingen gen̈troduceerd waarin vermogenselektronica met stuurschakelingen monolithisch geïntegreerd werden. Deze zogenaamde "Power Integrated Circuits" (PIC) maakten het mogelijk om vermogensystemen zoals spanningsregulatoren en vermogenversterkers veel goedkoper te produceren. In de jaren daarna zijn PICs verbeterd; ze worden tegenwoordig gebruikt voor veel verschillende toepassingen, waaronder in audioversterkers, motoraansturingen en elektronica voor automotive toepassingen. De omzet gegenereerd door dit soort elektronica werd in 2016 geschat op 10 miljard US dollar.

Tegelijkertijd met de door de "Wet van Moore" beschreven voortdurende vooruitgang in traditionele Silicium (Si) Complementaire MetaalOxide-Halfgeleider (CMOS) technologieën, waardoor we steeds meer rekenkracht krijgen voor een steeds lagere prijs, zijn er ook speciale CMOS technologieën ontwikkeld voor PICs om "Meer-dan-Moore" toepassingen mogelijk te maken. In deze (meestal) Bipolaire-CMOS-DMOS (BCD) technologieën, die ook bekend staan als "Smart Power Integrated Circuit" technologieën, wordt "slimheid" van digitale schakelingen gecombineerd met de "power" van vermogenselektronica.

In veel systemen in Smart-Power technologieën is een galvanische scheiding nodig vanwege ofwel veiligheidsredenen ofwel om interferentie te voorkomen bij datacommunicatie tussen verschillende spanningsdomeinen. Communicatie door middel van licht tussen de verschillende spanningsdomeinen is dan aantrekkelijk. De reden hiervoor is dat bij datatransmissie via licht er geen enkel elektrisch geleidend pad tussen verschillende (delen van) schakelingen nodig is en omdat een dergelijke verbinding immuun is voor externe elektrische en magnetische signalen.

Het principe van datacommunicatie met licht tussen galvanisch gescheiden delen van een elektronisch systeem wordt gebruikt in de zogenaamde "optocouplers". In optocouplers wordt licht uitgezonden door de lichtbron in het ene spanningsdomein en wordt dat gedetecteerd door een ontvanger in een ander spanningsdomein. Tegenwoordig zijn alleen discrete optocouplers beschikbaar; deze de discrete componenten verhogen de kosten voor PICs echter behoorlijk. Een monolithische implementatie van optocouplers - zonder extra productiestappen, dus in standaard CMOS zou een grote stap voorwaarts zijn waarmee verscheidene nieuwe "smart" PICs tegen lagere kosten en met een kleiner oppervlakte mogelijk worden. 
Het onderzoek naar deze geïntegreerde optocouplers is de focus van het onderzoek beschreven in dit proefschrif. De belangrijkste eisen van deze geïntegreerde optocouplers zijn: klein oppervlakte, lage energie-per-bit, hoge datasnelheid en de "hoeveelheid" isolatie tussen twee spanningsdomeinen.

Het belangrijkste probleem voor de monolithische implementatie van optocouplers is de afwezigheid van een efficiënte lichtbron in CMOS technologieën. Aangezien Si een halfgeleidermateriaal is met een indirecte bandgap van ongeveer $1,2 \mathrm{eV}$, genereert een licht-uitzendende-diode (LED) in silicium infrarood licht met een lage efficiëntie. Hieraan gerelateerd heeft een Si fotodetector (PD) een lage responsiviteit bij dergelijke golflengtes. Si LEDs in avalanche (AMLEDs) genereren daarentegen een relatief breed spectrum in het zichtbare gebied, met een grote overlap met de responsiviteit van Si PDs. In dit proefschrift wordt daarom gebruikgemaakt van AMLEDs als lichtbron voor monolithische optocouplers. Een ander probleem van Si AMLEDs is de relatief lage efficientie waarmee elektrische energie naar optische energie wordt omgezet (kwantum efficiëntie). Om de lage kwantum efficiëntie te compenseren, wordt een "single photon avalanche diode" (SPADs) in CMOS gebruikt voor de lichtdetectie. Zoals de naam suggereert, zijn SPADs heel gevoelige PDs, die (theoretisch) zelfs individuele fotonen kunnen detecteren.

In het eerste deel van dit proefschrift wordt in detail de fysica van avalanche-diodes besproken, wat belangrijk is om de prestaties van AMLEDs en SPADs te begrijpen. Er zal worden aangetoond dat avalanche kan worden beschreven door zogenaamd random-telegraph-ruis (RTS) in de diodestroom. Gebruikmakend van de gepresenteerde analyse van RTS kan de stroom-spannigskarakteristiek (I-V) worden verklaard en kan een eenduidige definitie worden gegeven van de zogenaamde breakdown-spanning. De meeste huidige Quenching-and-Recharge (QRCs) voor SPADs worden ontworpen met behulp van vuistregels. De analyse van RTS kan gebruikt worden om de QRCs beter te ontwerpen waardoor de snelheid van SPADs verhoogd kan worden met een factor 3 tot 6 in vergelijking met conventionele ontwerpstrategiën. De RTS-analyse is ook belangrijk voor het ontwerp van snelle AMLEDs: er volgt dat diodes met meer defecten en/of een hogere lekstroom resulteren in een sneller gedrag; dit is precies het tegenovergestelde als voor SPADs.

Het tweede gedeelte van dit proefschrift introduceert AMLEDs met geïntegreerde aanstuurelektronica in een $140 \mathrm{~nm}$ SOI CMOS technologie. Deze zuinige LED-aanstuurschakeling is inherent robuust tegen spreiding in zowel AMLED-eigenschappen als in werkomstandigheden van de AMLEDs. De gepresenteerde geïntegreerde lichtbron met aanstuurelektronica zorgt ervoor dat een lage energie-per-bit bereikt wordt in optische links. Als laatste demonstreert het werk in dit proefschrift voor het eerst een monolithische geïntegreerde optische link, met een klein oppervlakte (< $0.01 \mathrm{~mm}^{2}$ ) in een standaard CMOS technologie, met een datasnelheid van enkele Mbps bij een energieverbruik van enkele $\mathrm{nJ} / \mathrm{bit}$. 
Alles bij elkaar beschrijft dit proefschrift de fysica en toepassingen van avalanchediodes voor optocoupling-toepassingen. Meerdere fysicagerelateerde problemen voor avalanchediodes zijn besproken, die belangrijk zijn voor het ontwerp van AMLEDs, SPADs en de hun aanstuur- en uitleesschakelingen. De resultaten van het werk in dit proefschrift zijn veelbelovend en zorgen ervoor dat de droom van monolitische optocouplers nu dichterbij realisatie is dan ooit tevoren. 



\section{NOTE TO THE READER}

The chapters numbered two to six are based on peer-reviewed publications. They have been ordered in this thesis in a way that suits the flow for the general reader; from device physics to design, optimization, and finally to the application. This order does not necessarily match the chronological order in which they were published. In addition, because of being an independent publication, each of these chapters contains its own introduction. The reader is thus not bound to read all the chapters preceding the one he/she is particularly interested in. However, for a reader who is not properly acquainted with the topic, it is recommended to read the chapters in order.

Vishal Agarwal 



\title{
CONTENTS
}

\author{
ABSTRACT · vii \\ SAMENVATTING · ix \\ 1 INTRODUCTION · 1
}

1.1 Introduction to optical communication . 1

1.2 Applications of optocoupling · 4

1.3 Evolution of applications using light emission from silicon · 6

1.4 Scientific Challenges . 9

1.5 Optocoupling-in-CMOS project results summary · 11

1.6 Thesis outline $\cdot 12$

2 Analysis of Random Telegraph Signal PhenOMENA IN AVALANCHE DIODES $\cdot 15$

$$
\begin{aligned}
2.1 \quad \text { Introduction } & \cdot 16 \\
2.2 \text { Experimental Setup } & \cdot 17 \\
2.3 \text { Time Domain Analysis Procedure } & \cdot 19 \\
2.4 \text { Bias dependent RTS parameters } & \cdot 20 \\
2.5 \quad \text { Applications } & \cdot 24 \\
2.6 \text { Conclusions } & \cdot 26
\end{aligned}
$$$$
2.4 \text { Bias dependent RTS parameters · } 20
$$

3 RTS PHENOMENA IN ULTRA-SHALLOW SILICON AVALANCHE DIODES $\cdot 27$

$$
3.1 \text { Introduction } \cdot 28
$$

3.2 Experimental setup $\cdot 29$

3.3 RTS characterization · 32

3.4 Bias dependent RTS parameters · 35

3.5 Estimation of model parameters . 36

3.6 Conclusions $\cdot 44$

4 DATA TRANSMISSION CAPABILITIES OF SILICON AVALANCHE MODE LEDS · 47

$$
4.1 \text { Introduction · } 48
$$

4.2 Experimental AMLEDs and noise · 48

4.3 Pulse position modulation speed · 51

4.4 Design recommendations · 61 


\subsection{Conclusions · 62}

5 OPTICAL TRANSMITTER USING AVALANCHE LEDS IN SOI CMOS TECHNOLOGY $\cdot 65$

5.1 Introduction $\cdot 66$

5.2 Optoelectronic properties of the AMLED $\cdot 67$

5.3 Optical link transmission efficiency, $\eta_{\mathrm{TE}} \cdot 69$

5.4 AMLED driver circuit for an optocoupler · 72

5.5 Measurement results $\cdot 74$

5.6 Application in opto-couplers $\cdot 80$

5.7 Conclusion $\cdot 82$

6 Optocoupling IN CMOS · 83

6.1 Introduction $\cdot 84$

6.2 Experimental Devices · 85

6.3 Optical Link Performance · 89

6.4 Conclusions . 94

7 CONCLUSIONS AND RECOMMENDATIONS · 97

BIBLIOGRAPHY $\cdot 103$

A Multiplication nOise in ON-STATE $\cdot 113$

B DistribUtion OF PEAK CURRENT $\cdot 115$

C Light EMission PROFILES ·117

LIST OF PUBLICATIONS $\cdot 119$

ACKNOWLEDGEMENTS $\cdot 121$ 


\section{CHAPTER}

\section{INTRODUCTION}

\subsection{Introduction to optical communication}

On the invention of the photophone [1], Alexander Graham Bell said that it is "the greatest invention [I ever] made, greater than the telephone". In the photophone, Bell and his colleagues transmitted sound on a beam of light over a distance of about $200 \mathrm{~m}$. At present, the concept of data communication using light is ubiquitous, e.g. fiber-optic communication employed in telecommunication, the internet and television. The range of applications enabled using optical communication is a testimony to Bell's beliefs about the photophone.

Dedicated research was conducted to enable the use of light for communication since the middle of the 20th century. As a result, optical communication have significantly evolved compared to the photophone. The arrival of semiconductor lasers [2-4], the discovery of low attenuation fibers [5] and continuous advances in the silicon $(\mathrm{Si})$ technology [6-8] enabled a number of applications employing optical communication.

The progress in optical communication has been possible by the virtue of some interesting properties of light: it can propagate at an astounding speed, it is not "bothered" by external interferences, it is difficult to tap, it can be "guided" using fibers, and at some wavelengths it can be transmitted with very low losses through an optical fiber [9].

Light can also be used for data communication between circuits operating in two voltage domains, thus galvanically isolating the two domains from one another. This property has been utilized in so-called "optocouplers" (Fig. 1.1) [10]. At present, only discrete optocouplers are available which employ compound semiconductor devices (e.g. GaAs in IL4208 from Vishay semiconductors [11]) as optical transmitters; optical receivers can be implemented in Si complementary metal-oxide-semiconductor (CMOS) technologies. This hybrid integration makes the processing more complex 


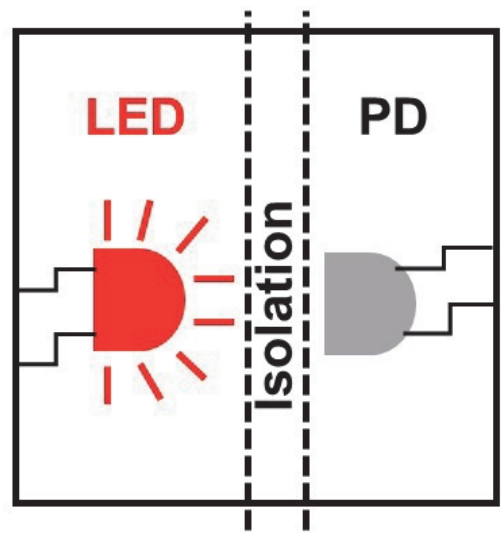

Figure 1.1: Schematic of an optocoupler. Light emitted by a light-emitting diode (LED) is detected by a photodetector (PD) across an electrical isolation barrier.

and thereby more expensive. To enable the implementation of optocouplers in $\mathrm{Si} \mathrm{CMOS} \mathrm{technologies} \mathrm{has} \mathrm{been} \mathrm{the} \mathrm{focus} \mathrm{of} \mathrm{the} \mathrm{research} \mathrm{in} \mathrm{this} \mathrm{thesis.}$

At present, optocouplers have not been implemented in CMOS technologies. The main reason is the mismatch between the emission spectrum of a forward biased Si LED and the responsivity of an Si photodetector (PD). Forward biased Si LEDs emit light in a relatively narrow range in the infrared region (wavelength $900 \mathrm{~nm} \leqslant \lambda \leqslant 1150 \mathrm{~nm}$ ) with low efficiencies due to an indirect band gap [12]; Si PDs have a relatively low responsivity at those wavelengths. This results in a poor optocoupling efficiency $(\eta)$ between a forward biased Si LED and an Si PD [13] (Fig. 1.2(a)).

An interesting property of $\mathrm{Si}$ diodes operating in avalanche breakdown is the visible light emission from these diodes [14]. During avalanche, charge carriers in the depletion region are accelerated due to a very high electric field $\left(10^{5}-10^{6} \mathrm{~V} / \mathrm{cm}\right)$ attaining high energies, higher than the band gap of $\mathrm{Si}(1.1 \mathrm{eV})$. Many of these accelerated carriers cause impact ionization to generate extra carriers which are further accelerated. Some of these high energy carriers recombine and because of these recombination events, high energy photons (with lower wavelengths) are emitted. A broad emission spectrum in the $400-850 \mathrm{~nm}$ range is obtained from these avalanching diodes [13-17]. At those wavelengths, there is a significant overlap with the responsivity of the Si PDs, as illustrated in Fig. 1.2(b). This overlap increases the $\eta$ between an Si avalanche mode LED (AMLED) and an Si PD compared to the $\eta$ between a forward biased Si LED and an Si PD (Fig. 1.2(a)) [13]. A high $\eta$ is essential for the implementation of optocouplers in CMOS technologies [13, 15-17].

The integration of optocouplers in standard CMOS technologies would be cost effective due to a relatively low cost of fabrication and low area requirements [16]. Due to monolithic integration, circuit parasitics can 


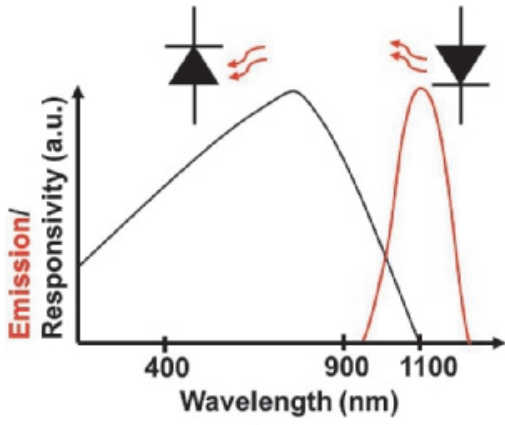

a)

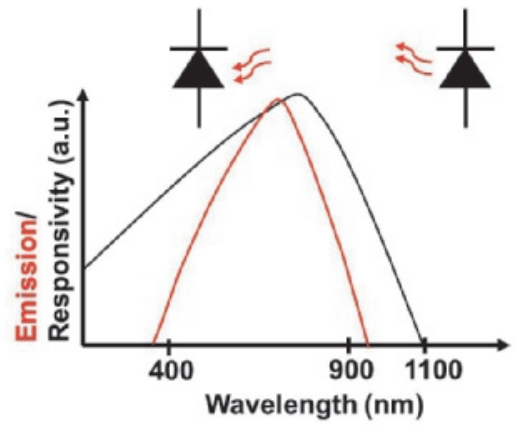

b)

Figure 1.2: Schematic plots to illustrate (a) poor match between the emission spectrum of a forward biased Si LED and responsivity of an Si photodetector, (b) overlap between the emission spectrum of an avalanche mode Si LED and the responsivity of the same Si photodetector.

also be reduced and therefore the propagation delay can be lower, when compared to discrete implementations of optocouplers. Higher levels of system integration are also possible which are advantageous for many applications [16]. Many new "smart" chips are possible; the research on these "smart" chips is referred to as "Silicon CMOS photonics" [18].

Further, the isolation layer (Fig. 1.1) in a CMOS integrated optocoupler can be implemented via oxide layers such as trench isolation layers, which can sustain a relatively high breakdown field $(\sim 800 \mathrm{~V} / \mu \mathrm{m})$ [19]. In comparison, the mold compounds used in traditional discrete optocouplers have a lower breakdown field $(\sim 50 \mathrm{~V} / \mu \mathrm{m})$ [19]. A high breakdown field $\left(E_{\mathrm{B}}\right)$ results in a high breakdown voltage $V_{\mathrm{B}}=-E_{\mathrm{B}} \cdot W$, where $W$ is the width of the corresponding isolation layer. A high $V_{\mathrm{B}}$ is important in many safety applications [20]. For intra-chip optical communication, the isolation can be implemented via traditional oxide layers of a CMOS technology, e.g. the Medium Trench Isolation. Back end oxides can be used as an insulating material for inter-chip optical communication.

Although visible light emission from AMLEDs has been known for about half a century (section 1.3), a commercial application for adopting AMLEDs is still missing. There are some possible reasons for this. Firstly, the efficiency of light emission, also referred to as the internal quantum efficiency (IQE) of Si AMLEDs is relatively low, in the order of $10^{-5}$ [15]. In other words, for every $10^{5}$ electrons flowing through the diode, on average 1 photon is emitted. Secondly, because of the low IQE of the AMLEDs, high quality ultra-sensitive PDs are required as the optical receivers in $\mathrm{Si}$ technologies; such PDs have been available only recently. A reason could be that avalanche has been avoided as an operating regime because of the usually associated high power consumption. Lastly, it is possible that researchers lost the track of this idea after light emission from compound semiconductors were discovered [2]. Compound semiconductors can emit 


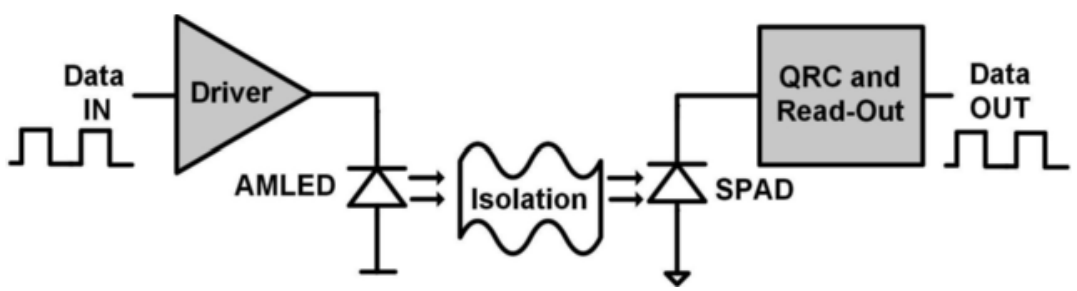

Figure 1.3: Overview of the proposed optocoupler in CMOS technology: optical transmitter (AMLED), optical receiver (SPAD), isolation barrier and the required circuits can be monolithically integrated.

light in the visible and near infrared wavelengths in forward mode at high efficiencies because of their direct bandgap [21].

The progress in the ultra-sensitive Si PD technology has made the dream of monolithic CMOS integration of optocoupling feasible now. Due to the low IQE of the AMLEDs, of special interest are the single photon avalanche diodes (SPADs) in CMOS technology [22]. In principle, SPADs are $\mathrm{p}-\mathrm{n}$ junction diodes biased above the breakdown voltage $\left(V_{\mathrm{BR}}\right)$ of the diode. At those bias conditions, the electric field is very high and theoretically a single incident photon can trigger an avalanche event; the avalanche current then swiftly increases to macroscopic values which can be detected using simple readout circuits [23]. Suitable quenching-and-recharge circuits (QRCs) are employed to quench the avalanche once triggered and reset the SPAD for further photon detection [22].

The idea of using avalanching junctions for single photon detection has been present since the origin of Si technology [24-29], however the progress was limited by the defects in devices caused during fabrication [29]. These defects caused a high dark count rate (DCR) and therefore the devices could not be reliably used for single photon detection. The introduction of the epitaxial layer resulted in high quality junctions with low defect density [29]. SPADs with a timing accuracy of tens of picoseconds (unreported DCR) were demonstrated as early as 1988 [30]. Since then, significant progress has been made; high quality ultra-sensitive SPADs in standard CMOS technologies with DCR of few $\mathrm{Hz}$ are now commercially available and used in several applications [31-33]. The ultra-sensitivity of these SPADs can be used to mitigate the low IQE of the AMLEDs.

Fig. 1.3 represents the schematics of the proposed optocoupler in this thesis.

\subsection{Applications of optocoupling}

An example of application of galvanic isolation in a medical system is shown in Fig. 1.4, where a barrier is required between the medical instruments and the patients. The isolator must protect the patients from 


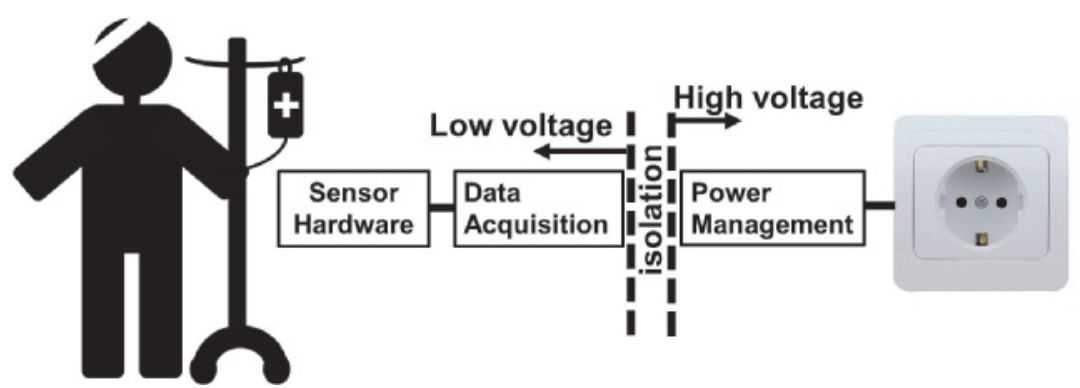

Figure 1.4: An illustrative example of a medical system including required galvanic isolation [20].

hazardous voltages and currents while the sensors gather essential patients data. Optocouplers as isolators are attractive for such applications.

In industrial environments, potential applications are gate driver circuits employed in motor drives and solar inverters (Fig. 1.5) [34]. In these circuits, the gate driver operating at $V_{\mathrm{CC}}$ must be isolated from the load side which operates at $V_{\mathrm{DC}}$ (tens or hundreds of volts). Another potential application is the monitoring of a high-voltage measurement system.

Integrated optocouplers are also attractive for the so-called "smart power" integrated circuit (IC) technologies such as Bipolar-CMOS-DMOS $(\mathrm{BCD})[35,36]$. These smart power technologies combine high voltage transistors with low voltage digital circuits which enables monolithic integration of high power applications such as audio amplifiers and automotive systems. In many of these applications, communication between two voltage domains while maintaining a galvanic isolation is required; this isolated communication link is enabled by an isolator.

The important performance metrics of such an isolator are:

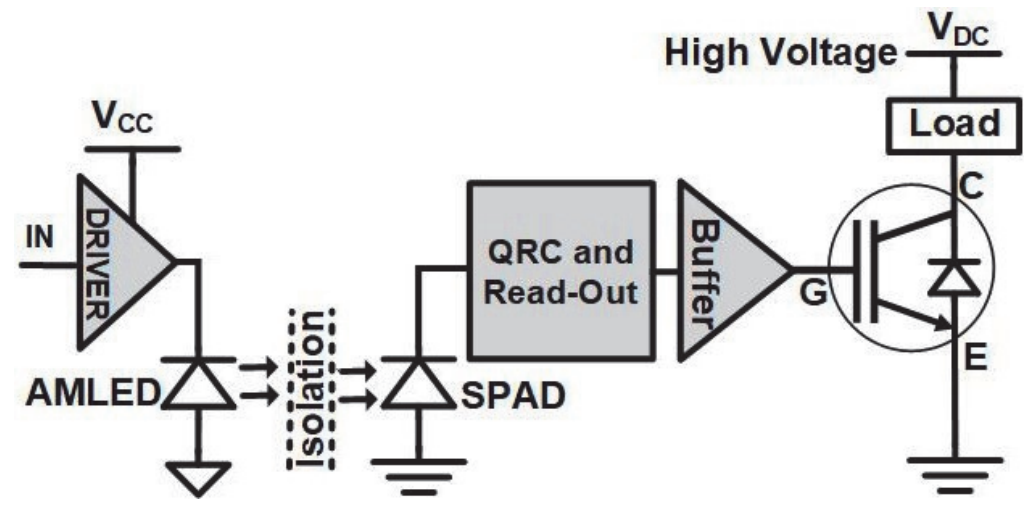

Figure 1.5: Schematic of an optically isolated gate driver for an insulatedgate bipolar transistor (IGBT). Similar driver circuit can be employed for a power MOSFET. 
1. Isolation performance: The maximum continuous voltage difference between two voltage domains that an isolator can tolerate is denoted as the "working isolation voltage" ( $\left.V_{\text {ISO }}\right)$, usually expressed in $\mathrm{kV}$ [37]. Isolation is also measured in terms of common mode transient immunity (CMTI), expressed in $\mathrm{kV} / \mu \mathrm{s}$ [37]. A high-frequency, high amplitude transient at one voltage domain can couple to an another voltage domain capacitively; this coupling can corrupt the data transmission across an isolator. CMTI is a measure of an isolator's capability to "tolerate" such transients.

2. Data rate: For data communication applications, a high data rate with a low bit-error-rate (BER) is required [37].

3. Energy-per-bit: This is the measure of the power consumption of an isolator and an important figure-of-merit in data communication applications [38].

4. Area: The area occupied by an isolator in a system. A small area implementation results in a low cost for the system.

Currently, integrated isolators are also implemented using capacitors or inductors $[39,40]$. Capacitive isolators require capacitors to be implemented in the back end and can be significantly large when isolating large voltage domains (e.g. $3 \mathrm{~mm}^{2}$ [39]). Inductive isolators are also usually large, e.g. $>30 \mathrm{~mm}^{2}$ [40]. Integrated optocouplers can enable such galvanically isolated optical links at a lower area requirements (e.g. $\sim 0.05 \mathrm{~mm}^{2}$ [41]). The performance details of the [39] and [40] are summarized in Table 1.1. Now, the evolution of applications using light emission from silicon is described.

\subsection{Evolution of applications using light emission from silicon}

There has been interest in exploring the use of visible light emission from Si avalanche diodes since the mid 1950s. In this section, the salient features of some of these works are highlighted. The focus is only on the crystalline Si junctions which are compatible with standard CMOS processes.

Visible light from Si p-n junctions operating in avalanche was first reported by Newman in 1955 [14]. He showed the relatively wide spectrum of the emitted light from avalanching Si junctions, when compared to the emission spectrum of a Si junction operating in forward mode. As stated in section 1.1, the wide spectrum was explained to be caused by the radiative recombination of high-energy carriers during avalanche breakdown.

Around the same time, the instability in the current-voltage characteristics during avalanche breakdown was reported [42]. At the onset of avalanche, current flows in the form of unstable pulses of random duration. An extensive research on this instability phenomenon was done over for 
almost a decade [42-52]. It was shown that at crystal defects in p-n junctions, the field is enhanced and the breakdown starts to occur [49]. The breakdown at these local defects is not stable and this causes avalanche current to flow as unstable pulses of random durations. These localized defects were also shown to be the spots from where the light was emitted in the diodes [46]. The term "microplasma" for these defects is originating from their light emitting nature. The light emission from avalanche breakdown served as a great characterization tool in the 1960s for the characterization of defects causing breakdown $[46,47,50,51]$. In recent years, this light emission has also been used in photo emission microscopy (PEM) for reliability analysis of Si-based circuits and devices to investigate the breakdown locations and the destruction mechanisms [53, 54].

In 1965, Haitz reported optical coupling between two different diodes fabricated on a single substrate [55]. The photons emitted from one of the "microplasmas" in one diode increased the pulse rate during instability in the other diode. Currently, this effect is well-known in the context of SPADs as "crosstalk" [56].

Probably the first consumer application idea for making use of light emission from avalanching junctions in Si was conceived by Kabell (1968) [57]. He used these light sources for the purpose of photographic data recording. The potential of avalanche diodes for VLSI interconnects was discussed by D. Kerns et al. in 1989 [58]. In an experiment [58], the light emitted by an AMLED was guided with an optical fiber and coupled to a nearby PD. The light from the AMLED was modulated at a few $\mathrm{kHz}$ and a corresponding output at the receiver was observed. The power consumption, data rate and any BER performance were not reported. However, the importance of AMLEDs in implementing cost effective on-chip interconnects was highlighted. It was argued that although the light output power is small, it could be sufficient for short interconnections. Although the system was not fully integrated, the experimental demonstration of optocoupling between two Si devices kindled the interest of many researchers.

In a partly experimental work in 1992 [17], Drieënhuizen et al. discussed the idea of using AMLEDs for optocoupling applications. Their interests were similar to those of other researchers, i.e. monolithic integration of optocouplers in standard technologies. In an experiment, Drieënhuizen et al. showed optocoupling between an AMLED and a PD on the same substrate. The authors argued that their design was limited by the poor optocoupling efficiency of the waveguide between the AMLED and the photodetector. Circuits for implementation of optocouplers in standard Si technology were proposed. However, no performance data on data communication or power consumption were reported and an experimental realization of the proposed system was not demonstrated.

At this point in the timeline, the extensive research done by Snyman et al. between 1996-present is important to consider [18, 59]. Their research has been focused on improving AMLED structures to emit at submicron 
wavelengths with high efficiencies and to design CMOS compatible waveguides at those wavelengths. This research has resulted in efficient and reliable AMLEDs at submicron wavelengths. The use of dielectric layers in CMOS such as the field oxide, intermetallic oxide and silicon nitride as a waveguide has been demonstrated $[59,60]$. Snyman et al. have also demonstrated a "proof of concept" of optical coupling in systems utilizing solely Si components [60]. In their optical link [60], the authors reported that signals of 60-100 nA could be observed at the PD when the AMLED was driven with 0-20 V pulses. In that work [60], the frequency of operation, BER, power consumption or isolation voltage have not been reported.

Another series of work done on Si AMLEDs is by Chatterjee et al. between 2002 - 2004 [61-63]. In a reliability study [61], the authors stressed AMLEDs with (a) a DC excitation, (b) an AC excitation and (c) a high temperature environment for accelerated aging. The measurements were done for 1 month at each stress condition. (a) The DC excitation at low values of current (less than $25 \mathrm{~mA}$ ) caused a light coalescence phenomenon (merging of light-emitting regions), however the total light emission intensity remained constant. At high values of DC currents, not such coalescence in light emission was observed. The differences in the light emission at low and high values of DC currents were explained using a hydrogen migration model. (b) For the AC excitation, a $10 \mathrm{~V}$ peak-to-peak voltage signal at 10 MHz superposed on a 5V DC was applied at the AMLED, for which the $V_{\mathrm{BR}} \sim 6.5 \mathrm{~V}$. (c) For accelerated aging, the AMLEDs were placed in a $100^{\circ} \mathrm{C}$ environment. It was reported that the $\mathrm{AC}$ and temperature stressing also had negligible effects on the light emission from AMLEDs.

In [62], Chatterjee et al. demonstrated an inter-chip optical link using Si AMLEDs. The light emitted by an AMLED of a size of $75 \mu \mathrm{m} \times 75 \mu \mathrm{m}$ was coupled through a 36" long $\mathrm{SiO}_{2}$ fiber cable of a $50 \mu \mathrm{m}$ diameter to an Si PD. Also, the light emission from the AMLED was modulated at $100 \mathrm{kHz}$ using a sinusoidal input and a corresponding modulation in the output of the PD was observed. The authors stated that the modulation speed could not be improved because of the low bandwidth of the PD. The power consumption of the transceiver circuits were not reported there. In [63], the voltage across an AMLED ( $\left.V_{\text {AMLED }}\right)$ was modulated at frequencies in the $\mathrm{GHz}$ range and the temporal behavior of the light emission was observed using a high speed streak camera (Hamamatsu C4780). In one measurement setting, the $V_{\text {AMLED }}$ was modulated using a sinusoidal signal of frequency $1 \mathrm{GHz}$ and the output of the camera was recorded. In an another setting, the AMLED was driven by a voltage pulse with a pulse width of $2 \mathrm{~ns}$ and an amplitude of $5 \mathrm{~V}$. Parasitics in the circuit and the heavy impedance mismatch generated high-frequency oscillatory signals in the range of $20 \mathrm{GHz}$ with a modulation index of $\sim 50 \%$. From the output of the streak camera, the authors demonstrated the modulation speed of Si AMLEDs in the range of tens of GHz which is very attractive for applications such as optical interconnects. 
In 2011, Huang et al. demonstrated an optocoupler in a standard 0.35 $\mu \mathrm{m}$ CMOS technology [41]. The active area of their design was about 0.05 $\mathrm{mm}^{2}$ which highlights the low area implementation of integrated optocouplers. The salient feature is the monolithic integration of all components on a single substrate, while no technology modification or post-processing was done. Although no data communication was shown, that work highlights the successful integration of metallic waveguides and is yet another "proof of concept" for optical coupling in a standard CMOS technology.

In 2013, a monolithically integrated optical link utilizing both avalanche AMLEDs and SPADs was demonstrated for joystick applications where the operating frequency is about $100 \mathrm{~Hz}$ [64]. The area of the implemented chip in a $140 \mathrm{~nm}$ technology was about $2.5 \mathrm{~mm}^{2}$ and the power consumption was reported to be $\sim 5 \mathrm{~mW}$. The attractive idea of that work is the successful demonstration of incorporating SPADs for compensating the low internal quantum efficiency of AMLEDs (Section 1.1).

Table 1.1 summarizes the state-of-the-art of integrated optocouplers and digital isolators. Note that for the majority of these works, only a limited set of performance metrics are reported or could be estimated.

\subsection{Scientific Challenges}

The integration of AMLEDs and SPADs with the standard CMOS circuits goes along with various challenges.

Avalanche operation of these devices requires high voltage electronics. While using AMLEDs, the optical transmitter can be power hungry because of the avalanche operation. Therefore, one big challenge is that the circuits should be power efficient and achieve a low energy-per-bit while ensuring a sufficiently low BER. The avalanche process is dependent on the process, voltage and temperature [65], therefore the AMLED driver circuit should be robust to these variations while providing a sufficiently high signal to the receiver (SPADs) (Fig. 1.3).

Since SPADs operate in the Geiger mode, the LED driver circuit should be able to completely switch the AMLED between on and off conditions for proper functionality of SPADs. Although small-signal modulation speed of AMLEDs in the range of tens of GHz has been reported [63], the large signal switching speed was found to be low, in the range of $\mathrm{kHz}$ to $\mathrm{MHz}$ depending on the AMLED design (chapter 4). In this respect, a proper design of AMLEDs for achieving high speed is required.

At the receiver side, the SPADs should be efficient in terms of photon detection efficiency and should have a low DCR for a low BER. A low DCR is possible by a proper choice of layers for the active area and the guard ring in the SPAD design [66]. Moreover, the operation of SPADs is also affected by their quenching-and-recharge circuits (QRCs). Currently, these $\mathrm{QRCs}$ are designed using a few rules-of-thumb. More research on the physics of SPADs was required to understand and improve the operation 


\begin{tabular}{|c|c|c|c|c|c|c|c|}
\hline & {$[58]^{*}$} & {$[62]^{*}$} & {$[41]^{* *}$} & {$[60]^{* *}$} & {$[64]^{* *}$} & {$[39]^{+}$} & {$[40]^{++}$} \\
\hline Type & opto & opto & opto & opto & opto & Cap. & Ind. \\
\hline Technology $(\mu \mathrm{m})$ & - & 1.5 & 0.35 & 1.2 & 0.18 & 0.5 & 0.18 \\
\hline $\begin{array}{l}\text { AMLED Electrical } \\
\text { power }(\mathrm{mW})^{\#}\end{array}$ & - & - & 1274 & - & - & NA & NA \\
\hline $\begin{array}{l}\text { Optical power } \\
(\mathrm{nW})^{\#}\end{array}$ & - & - & 31.2 & $\sim 10$ & - & NA & NA \\
\hline $\mathrm{IQE}^{\#}$ & - & - & $10^{-7}$ & - & - & NA & NA \\
\hline $\begin{array}{l}\text { Coupling effi- } \\
\text { ciency \# }\end{array}$ & - & - & - & - & - & NA & NA \\
\hline Modulation type ${ }^{\#}$ & Pulse & Sine & - & Pulse & - & OOK & $\mathrm{OOK}$ \\
\hline $\begin{array}{l}\text { Modulation speed } \\
\#\end{array}$ & $\begin{array}{l}\text { few } \\
\mathrm{kHz}\end{array}$ & $\begin{array}{l}100 \\
\mathrm{kHz}\end{array}$ & - & - & $\begin{array}{l}100 \\
\mathrm{~Hz}\end{array}$ & $\begin{array}{l}640 \\
\text { Mbps }\end{array}$ & $\begin{array}{l}500 \\
\text { Mbps }\end{array}$ \\
\hline $\begin{array}{l}\text { Total Power con- } \\
\text { sumption }(\mathrm{mW})\end{array}$ & - & - & - & - & 5 & 180 & 82.5 \\
\hline $\begin{array}{l}\text { Energy-per-bit } \\
\text { (/bit) }\end{array}$ & - & - & - & - & $50 \mu \mathrm{J}$ & $\begin{array}{l}281 \\
\text { pJ }\end{array}$ & $\begin{array}{l}175 \\
\mathrm{pJ}\end{array}$ \\
\hline Bit-error-rate & - & - & - & - & - & - & - \\
\hline$V_{\text {ISO }}(\mathrm{kV})$ & - & - & - & - & - & 2.5 & 7.5 \\
\hline CMTI $(\mathrm{kV} / \mu \mathrm{s})$ & - & - & - & - & - & - & 50 \\
\hline Active area $\left(\mathrm{mm}^{2}\right)$ & - & - & 0.05 & - & 2.5 & $\sim 3$ & $>30$ \\
\hline
\end{tabular}

Table 1.1: Summary of state-of-the-art of integrated isolators.

\# AMLED electrical/optical power, IQE, coupling efficiency and modulation characteristics are relevant for the integrated optocouplers energy-perbit and speed (section 1.2, chapters 4,5).

* AMLED and PD were not monolithically integrated, ** AMLEDs and PDs were integrated. [64] used SPADs as PDs.

${ }^{+}$[39] implemented a capacitive isolator whereas ${ }^{++}$[40] reports an inductive isolator. NA implies "Not Applicable".

of SPADs. A proper design of QRCs also improves the dynamic range of SPADs and the SPADs can then be used for higher speed.

In other aspects of the same project (Optocoupling-in-CMOS), there were challenges related to the device physics and modeling of AMLEDs. For instance, the breakdown voltage of AMLEDs should be minimized without deteriorating IQE to reduce energy-per-bit. These issues have been addressed in [67]. It has been demonstrated that the breakdown voltage preferably should be about $6 \mathrm{~V}$ for maximum efficiency in terms of AMLED optical output power in response to input electrical power.

On top of everything, the major design constraint for the work in this thesis was that no modification to the CMOS process was allowed. In other words, all devices and circuits were required to be compatible with a specific CMOS technology. 


\subsection{Optocoupling-in-CMOS project results summary}

This research work has been accomplished within the framework of the Optocoupling-in-CMOS (OiC) project, sponsored by the Dutch scientific foundation (NWO-TTW). The aim of OiC was to enable technologies for the monolithic integration of optocouplers in standard CMOS technologies. This interdisciplinary research was carried out by two PhD students with the expertise from two groups: the Integrated Circuit Design (ICD) group focusing on the circuit design aspects of the project and the Integrated Devices and Systems (IDS) group focusing on the device physics, both in the department of Electrical Engineering at the University of Twente.

A number of results were obtained during the course of this interdisciplinary project. The main findings of the OiC project are briefly listed:

- An optoelectronic model for the light emission from Si AMLEDs was developed [67]. Using a physics-based model and experimental results, it was shown that a $V_{\mathrm{BR}}$ of about $\sim 6 \mathrm{~V}$ is the optimum for maximum efficiency from the AMLEDs in any technology. Below this optimum point, the efficiency of an AMLED decreases because of non-local avalanche phenomena. This result is important because this puts a constraint to the reduction of $V_{\mathrm{BR}}$ of AMLEDs.

- A new type of AMLED, referred to as the superjunction AMLED was designed in a standard $140 \mathrm{~nm}$ SOI CMOS technology [67]. For breakdown voltages of $\sim 25 \mathrm{~V}$ and higher, this superjunction AMLED shows a two fold increase in the IQE.

- Random Telegraph Signal (RTS) phenomena in the avalanche current were observed for diodes designed in a standard $140 \mathrm{~nm}$ SOI CMOS and a pure boron technology [68,69]. It was shown that RTS phenomena can describe the steep $I-V$ characteristics in avalanche breakdown. It also provides a well defined value of the experimental breakdown voltage. The importance of the RTS analysis in the accurate design of QRCs for SPADs was discussed. The time domain analysis of the RTS phenomena can be used to estimate the dimensions of the defects causing various RTS phenomena in avalanche diodes.

- The large signal modulation speed of AMLEDs was studied for diodes designed in a pure boron technology and a standard 140 nm SOI CMOS technology [70]. It was shown that the AMLED design is important to improve their speed. The RTS phenomena help to understand the device properties related to the speed of AMLEDs.

- A monolithic optical link in a standard 140 nm SOI CMOS technology was demonstrated [13]. It was shown that the optocoupling efficiency of a link employing AMLEDs is higher than that of a link employing forward mode LEDs. The thermal effects were found to be significant at low data communication rates in the range up to a few tens of $\mathrm{Hz}$. 
The thermal effects can be minimized by the integration of on-chip heat sinks and/or by adopting high frequency switching.

- A low power LED driver circuit was demonstrated [71]. This charge based driver circuit was integrated and designed in a standard 140 nm SOI CMOS technology. It was shown that the designed driver circuit is robust to many variations in the properties of the AMLEDs.

- Finally, according to our best knowledge, for the first time, a complete optical link for data communication in a standard CMOS technology has been achieved using SPADs [72]. The active area of the designed system is $<0.01 \mathrm{~mm}^{2}$, the data rate in the range of a few Mbps and the energy-per-bit of a few nJ/bit. The isolation is expected to be similar to capacitive isolators and transformers due to the use of similar technology layers for isolation.

\subsection{Thesis outline}

- Chapter 2: Random telegraph signal phenomena in avalanche diodes: Application to SPADs: This chapter, based on the work presented at European Solid - State Device Research Conference (ESSDERC 2016) [68] shows the Random Telegraph Signal (RTS) phenomena in avalanche diodes fabricated in a standard $140 \mathrm{~nm}$ SOI CMOS technology. Using a time domain analysis of these RTSs, the steep $I-V$ characteristics in avalanche is explained, an experimental definition of the breakdown voltage is provided and the application of the RTS analysis in the accurate design of QRCs for SPADs is discussed.

- Chapter 3: Random telegraph signal phenomena in ultra-shallow $p^{+} n$ diodes: In this chapter, published in IEEE Journal of Electron Devices Society [69], the RTS phenomena in ultra-shallow junctions, fabricated using a pure boron technology were described in detail and the geometry dependence of the RTS phenomena have been analysed. Using a time domain analysis of these RTSs, the dimensions of the defects causing RTSs have been estimated.

- Chapter 4: Data transmission capabilities of avalanche mode light-emitting diodes: In this chapter (published in IEEE Transactions of Electron Devices) [70], the data transmission capabilities of AMLEDs fabricated in both pure boron and a standard $140 \mathrm{~nm}$ SOI CMOS technology has been investigated and results are explained using physically measurable AMLED parameters. Recommendations for the design of high speed AMLEDs are discussed as well.

- Chapter 5: Low power wide spectrum monolithically integrated optical transmitter in SOI CMOS technology: This chapter, based on our publication in Optics Express [71], demonstrates a low power optical 
transmitter in a $140 \mathrm{~nm}$ SOI CMOS technology. The demonstrated charge based LED driver circuit is robust to process, voltage, temperature and design variations. The driver circuit consumes a fixed energy-per-bit and emits a fixed number of photons per data bit.

- Chapter 6: Optocoupling in CMOS: In this chapter (presented at the IEEE Electron Devices Meeting 2018 [72]), monolithically integrated optocouplers in a standard $140 \mathrm{~nm}$ SOI CMOS technology are demonstrated. A low voltage AMLED with a breakdown voltage of $\sim 5$ $\mathrm{V}$ was designed to reduce power consumption of the link. The advantages of AMLEDs over forward biased Si LEDs are shown. The performance of the designed optical links was measured in terms of data rate and energy-per-bit.

- Chapter 7: Conclusions and recommendations for future work: In this chapter, the results of this thesis are summarized and recommendations for future work in this field are discussed. 



\title{
ANALYSIS OF RANDOM TElegraph Signal PhenOMENA IN AVALANCHE DIODES
}

\begin{abstract}
The current-voltage $(I-V)$ characteristics of diodes close to the breakdown voltage is shown to be governed by Random Telegraph Signal (RTS) phenomena. A technology independent time domain analysis method is used to accurately characterize the bias dependent statistical properties of these RTS phenomena and these are shown to explain the steep $I-V$ dependency in avalanche. Further, the statistical analysis of these RTSs is used for determining the self-sustaining avalanche current or latching current that is an important parameter in designing quenching and recharge circuits (QRCs) for single-photon avalanche diodes (SPADs). Accurate design of QRCs can improve the performance of SPADs in terms of count rates and afterpulsing. Based on these results, circuit simulations have been performed to show the advantages of applying the proposed method of adopting RTS-dependent latching current in the design of QRCs.
\end{abstract}

A major part of this chapter was presented at the European Solid-State Device Research Conference 2016 [68]. Simulation results have been added in section 2.5.1 for completeness sake. 


\subsection{Introduction}

The triggering phenomenon of avalanche in diodes has been described in $[25,73,74]$ (and references therein). Although most applications treat avalanche as the limiting region for using diodes, some applications explicitly make use of the avalanche region as the operating region. Major applications include optical detectors using avalanche photodiodes (APDs) [9] and single photon avalanche diodes (SPADs) [75]. Moreover, during avalanche, silicon ( $\mathrm{Si}$ ) diodes emit light at visible wavelengths, which is attractive for monolithic integration of optical links in CMOS technologies because of strong overlap of their emission spectrum with the responsivity of standard Si detectors [14, 16, 41, 76].

A relatively high electric field in the depletion region of $p-n$ junctions is used in APDs and SPADs. As the electric field is very high, an incoming free carrier, e.g. generated by a photon or otherwise, can trigger an avalanche event. The avalanche current then increases rapidly to large values $[12,75]$. APDs are typically operated in weak avalanche region, while SPADs are utilized in Geiger mode at a reverse bias $\left(V_{R}\right)$ beyond the breakdown voltage $\left(V_{\mathrm{BR}}\right)$. A high $V_{\mathrm{R}}$ in SPADs increases their photon detection efficiency and then they can be used to detect very faint optical signals. SPADs require a quenching and recharge circuit $(\mathrm{QRC})$ to be able to quench the avalanche once triggered and reset themselves for subsequent photon detection [22]. A low build up time of avalanche along with a high sensitivity results in SPAD sensors with excellent time resolution, which can be integrated with simple read out circuitry [22, 23, 77, 78]. SPADs have been used in many applications such as time-of-flight imaging and single-photon-emission computed tomography [23, 66, 79]. In this chapter, the focus is on the statistical behavior of the avalanche process and applications to SPADs.

In literature, for the quenching of avalanche, it is reported that the avalanche is self sustaining for diode currents $\left(I_{R}\right)$ higher than $100 \mu \mathrm{A}$, also sometimes denoted as the latching current $\left(I_{\text {lat }}\right)[22,52,66,80]$. Below this $I_{\text {lat }}$, it is reported that there is a high probability of quenching of avalanche [66]. In passive QRCs, as a rule-of-thumb, for determining the quenching resistance $\left(R_{\mathrm{Q}}\right)$, typically $50 \mathrm{k} \Omega$ per volt of excess bias $V_{\mathrm{EX}}=V_{\mathrm{R}}-V_{\mathrm{BR}}$ is used [22]. However, experimentally $V_{\mathrm{BR}}$ is not well defined and consequently $V_{\mathrm{EX}}$ is ill defined; therefore unique definitions for voltages that limit the steep $I-V$ part in avalanche are introduced in this chapter. It is shown that these generally accepted rules-of-thumb to estimate e.g. $R_{\mathrm{Q}}$ can result in an overestimation for high counting rate applications such as optical links and optical interconnects [38].

This chapter is outlined as follows. An experimental setup is described in section 2.2 that enables us to achieve very low external quenching, limited by the $50 \Omega$ input resistance of the measurement setup. This setup enables measuring currents with $160 \mathrm{nA}$ current resolution and 100 
ps time resolution which is sufficient to accurately measure and model avalanche Random Telegraph Signal (RTS) processes. Analyses show that $I_{R}$ near breakdown can be characterized as an RTS. A time domain method to analyze the RTSs is described in section 2.3. Section 2.4 presents the experimental results, the analysis of the RTSs and discusses the underlying statistics. These analyses allow to extract bias dependent statistical RTS properties such as expected values for the RTS amplitude and RTS On-time fraction as a function of $V_{\mathrm{R}}$. Combined, these are shown to fully describe the steep $I-V$ dependency in avalanche. Using the results, a parametric self-sustaining avalanche current level $\left(I_{\text {lat, }}\right)$ is defined in section 2.5 which enables accurate design of e.g. active or passive quench-and-recharge circuits. The advantages of the proposed method are also shown using circuit simulations. Finally, in section 2.6, the main findings of this chapter are summarized.

\subsection{Experimental Setup}

Fig. 2.1(a) shows a schematic cross-section of the diode in an industrial $140 \mathrm{~nm}$ SOI CMOS technology [36]. The SOI technology offers isolated voltage domains which is important for the implementation of optocouplers (chapter 1). A micrograph of the diode is shown in Fig. 2.1(b). A schematic layout of the experimental setup used to characterize diodes is also shown in Fig. 2.1(c). The avalanche multiplication region of the diode is beneath the $\mathrm{p}^{+}$region. In this setup, there are only non optical sources of free carriers to trigger avalanche in the diode: either from thermal generation, diffusion or defects in the multiplication region. However, optical sources (photons) can also trigger avalanche. Once triggered, the avalanche contribution $I_{\mathrm{A}}$ in the total diode current $I_{\mathrm{R}}$ flows until (actively, passively or self) quenched and only after that the diode reverts to its original non-avalanching state.

In this chapter, the main focus is on the characterization and modeling of self-sustaining properties of $I_{\mathrm{A}}$. For that reason, the total $R_{\mathrm{Q}}$ was minimized, here to only $50 \Omega$ of the measurement setup. This was accomplished by lowohmically biasing the cathode using a bias tee and by shunting the anode by the $50 \Omega$ input resistance of a high performance oscilloscope (Agilent DSO54854A). At low RTS current magnitude levels an additional highbandwidth low-noise amplifier (LNA) was used in front of the oscilloscope input; also this amplifier has $50 \Omega$ input resistance. A high data acquisition rate of $5 \mathrm{GS} / \mathrm{s}$ ensures that very narrow pulses could also be detected. This setup allows measuring currents with $160 \mathrm{nA}$ resolution with a noise floor of $0.4 \mathrm{nA} / \sqrt{\mathrm{Hz}}$. Measurements were done in a Faraday's cage in complete dark conditions at a temperature of $298 \mathrm{~K}$ with wafer probing. The data were acquired for a total duration of $1 \mathrm{~ms}$ at each bias condition.

Fig. 2.2 shows the DC $I-V$ characteristics as measured by a Source Measure unit (SMU) of a Keithley B2901A, using a $1 \mathrm{~s}$ integration time. 
a)

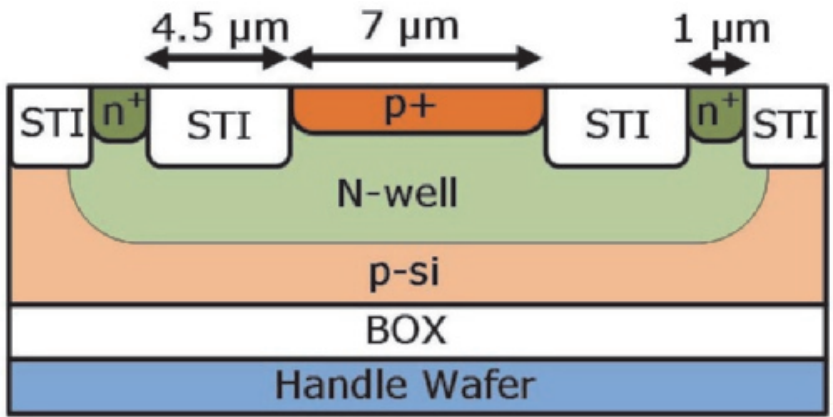

b)
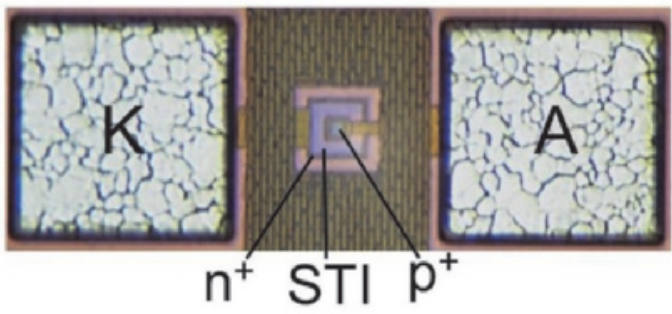

c)

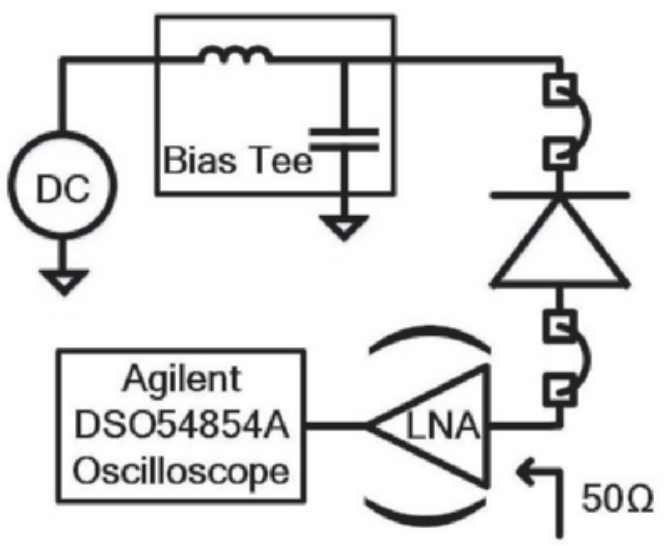

Figure 2.1: (a) Schematic cross-section of a square shaped $\mathrm{p}^{+} \mathrm{n}$ diode in a $140 \mathrm{~nm}$ SOI CMOS technology. (b) Micrograph of the designed diode. (c) Schematic layout of the experimental setup to measure the RTSs in avalanche diode.

In section 2.4 it is shown that the steep part of the DC $I-V$ curve is fully described by bias dependent statistical properties of the RTS underlying the avalanche process; also an exact determination of the experimental $V_{\mathrm{BR}}$ is given in section 2.4. The measurements indicate that the avalanche process starts around $14.7 \mathrm{~V}$ with $I_{\mathrm{R}}$ rising sharply between $14.8 \mathrm{~V}$ and 14.9 $\mathrm{V}$; this chapter is focused on that voltage range. 


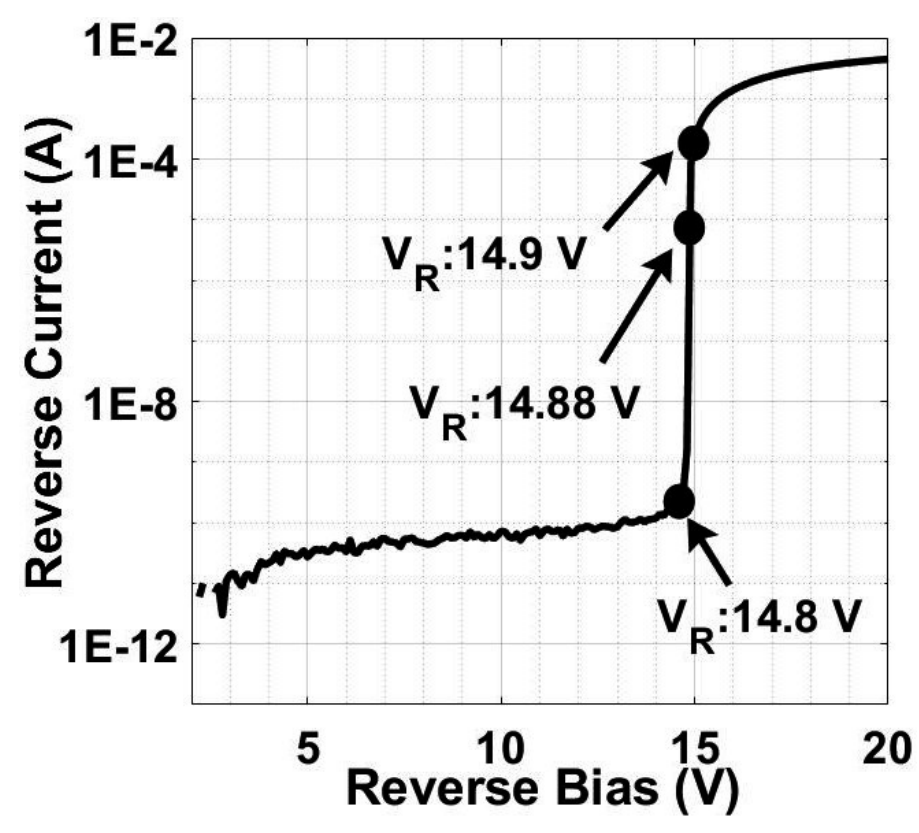

Figure 2.2: The DC characterized $I-V$ characteristics.

\subsection{Time Domain Analysis Procedure}

An example of measured $I_{R}$ for various $V_{R}$ is shown in Fig. 2.3. It can be observed that $I_{R}$ comprises current pulses with more or less two levels and a random duration. Further, the amplitude and duration of these pulses increase with $V_{\mathrm{R}}$. These current pulses were consistently observed among all samples and a similar increasing trend in amplitude and duration was recorded.

This type of RTS phenomena has been reported to be caused by the unstable microplasma behavior near $V_{\mathrm{BR}}[45,49,52,74]$. These microplasmas are formed at crystal imperfections where the electric field is increased above its average value [80]. However, a thorough time domain analysis (TDA) of the statistical parameters and their relation to the DC $I-V$ characteristics was not shown there.

Fig. 2.3 also shows that the avalanche process is not self-sustaining and switches between the on-state ("ON") and the off-state ("OFF"). This ON-OFF behavior can be described by RTS phenomena and can be characterized by a few parameters:

1. the expected on-state lifetime, $E\left(T_{\mathrm{ON}}\right)$,

2. the expected off-state lifetime, $E\left(T_{\mathrm{OFF}}\right)$, and

3. the amplitude difference between the states, $A$.

The power spectral density (PSD) of a two level RTS has a Lorentzian shape [81, 82]. Earlier, some of the RTS parameters were approximated 
a)
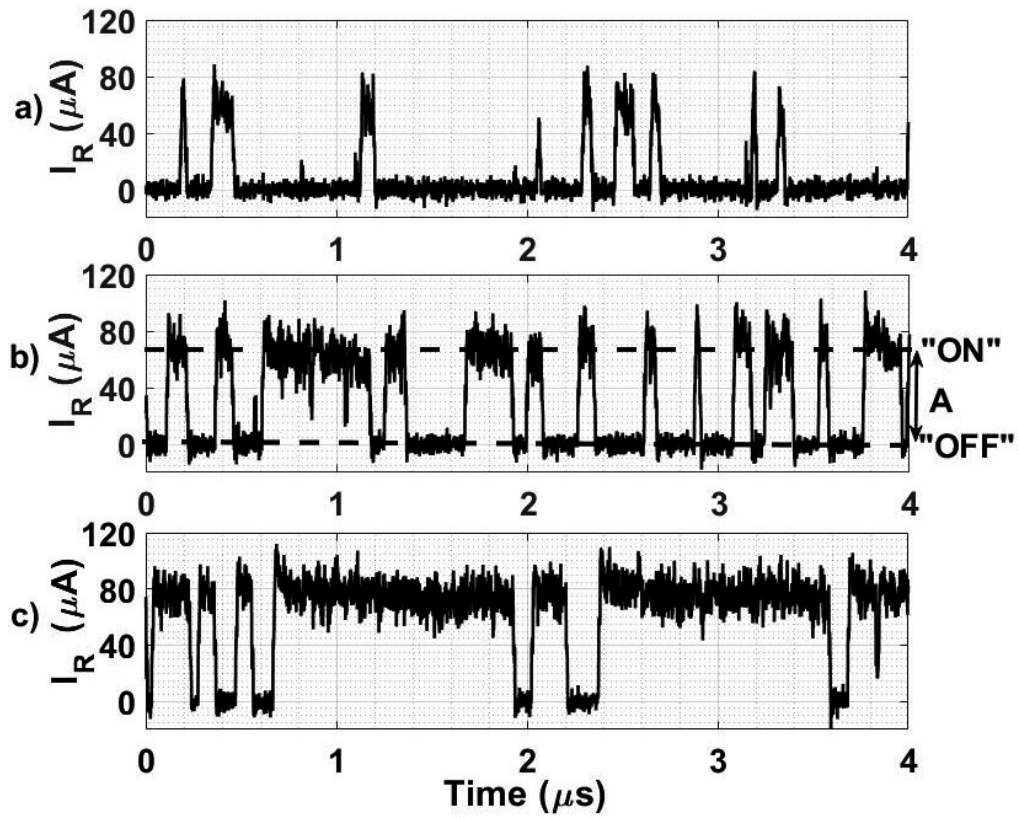

Figure 2.3: An example of the $I_{\mathrm{R}}$ at $V_{\mathrm{R}}$ : (a) $14.86 \mathrm{~V}$ (b) $14.88 \mathrm{~V}$ (c) $14.90 \mathrm{~V}$. $I_{\mathrm{R}}$ comprises pulses with almost a fixed amplitude and random duration. The pulse amplitude and duration increase for higher $V_{\mathrm{R}}$.

from the PSD [45]. In this work, the TDA is utilized to extract the RTS properties more easily.

The time domain current $I_{\mathrm{R}}$ is displayed as a histogram [83, 84]. Fig. 2.4 shows the histogram of $I_{R}$ at different $V_{R}$. The amplitude and the amount of time in the on-state increases with $V_{\mathrm{R}}$. Following parameters of the RTS can be estimated using a Gaussian fit (Fig. 2.4(b)): the mean values of the OFF-level $\left(b_{0}\right)$ and the ON-level $\left(b_{1}\right)$, the standard deviation of the OFF-level $\left(\sigma_{0}\right)$ and the ON-level $\left(\sigma_{1}\right)$. The RTS amplitude $A$ is estimated as $A=b_{1}-b_{0}$. In our measurement setup, $\sigma_{0}$ is mainly caused by the oscilloscope, while $\sigma_{1}$ also includes the multiplication noise [85].

To extract the time domain statistical parameters, $I_{R}$ is quantized into a two-level RTS using a simple level-crossing algorithm, similar to algorithms in e.g. data recovery in digital communication channels $[84,86]$. The measured $I_{R}$ is quantized into $I_{Q, R T S}$ as:

$$
I_{\mathrm{Q}, \mathrm{RTS}}= \begin{cases}A, & \text { if } I_{\mathrm{R}} \geqslant I_{\mathrm{TH}} \\ 0, & \text { otherwise. }\end{cases}
$$

\subsection{Bias dependent RTS parameters}

Using the procedure described in section 2.3, statistical properties of the RTSs were obtained for various $V_{R}$. In the context of avalanche processes 
a)

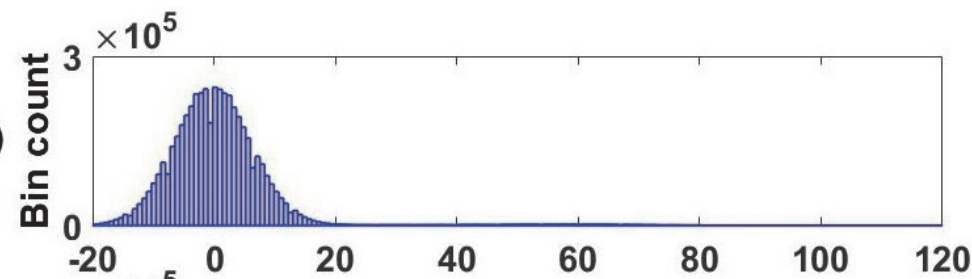

b)

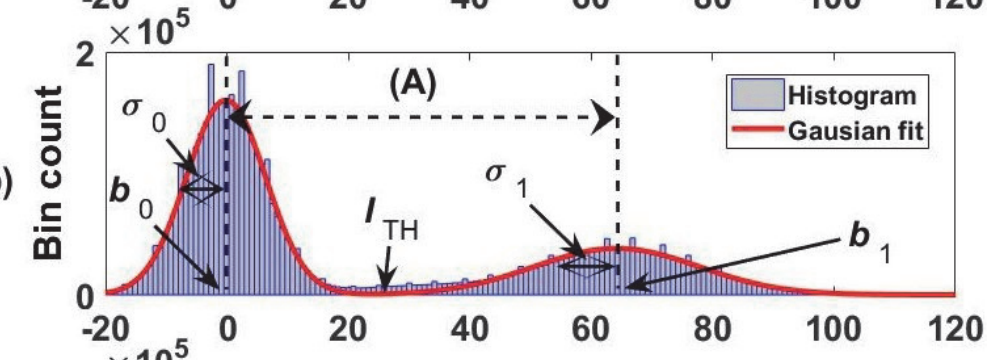

c)

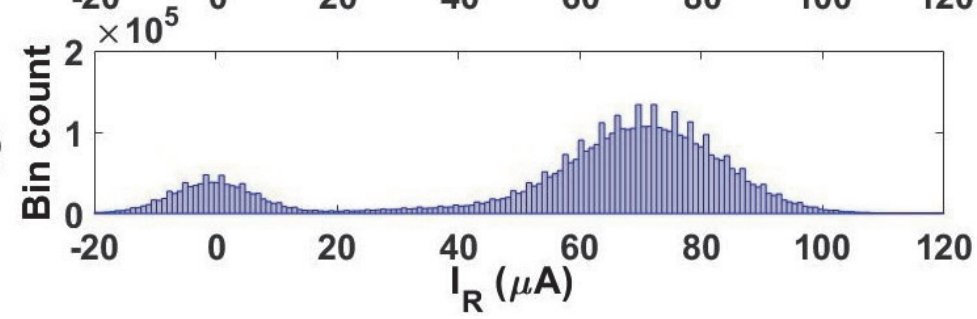

Figure 2.4: Histogram of $I_{\mathrm{R}}$ at $V_{\mathrm{R}}$ : (a) $14.86 \mathrm{~V}$ (b) $14.88 \mathrm{~V}$ (c) $14.90 \mathrm{~V}$. The increasing amplitude of the RTS can be observed. In (b), a Gaussian fit of $I_{\mathrm{R}}$ is shown with the OFF-level average $b_{0}$, standard deviation $\sigma_{0}$; the ON-level average $b_{1}$ and standard deviation $\sigma_{1} . I_{\mathrm{TH}}$ is used to quantize the observed RTS pulses into a two level RTS.

and SPADs, the inter-arrival times (IATs) between the RTS pulses, the pulse width of RTS pulses and the RTS amplitude are the most relevant. Also the standard deviation of these give information, but is dealt with in chapter 3 .

\subsubsection{Inter-arrival times}

From $I_{Q, R T S}$ IATs for the ON and OFF state pulses were calculated. An example of a measured probability density function (PDF) of the interarrival time for $V_{\mathrm{R}}=14.88 \mathrm{~V}$ is shown in Fig. 2.5; similar PDFs were obtained for other bias conditions in avalanche. These PDFs show that the IATs for the ON-state and OFF-state states are exponentially distributed which confirms that the observed RTS process is similar to a Poisson distribution [87]. The peak in the PDF at the far left hand side (indicated by an arrow) is because of the "afterpulsing" [23]. The conventionally used IAT for a certain state equals the lifetime for the other state. 

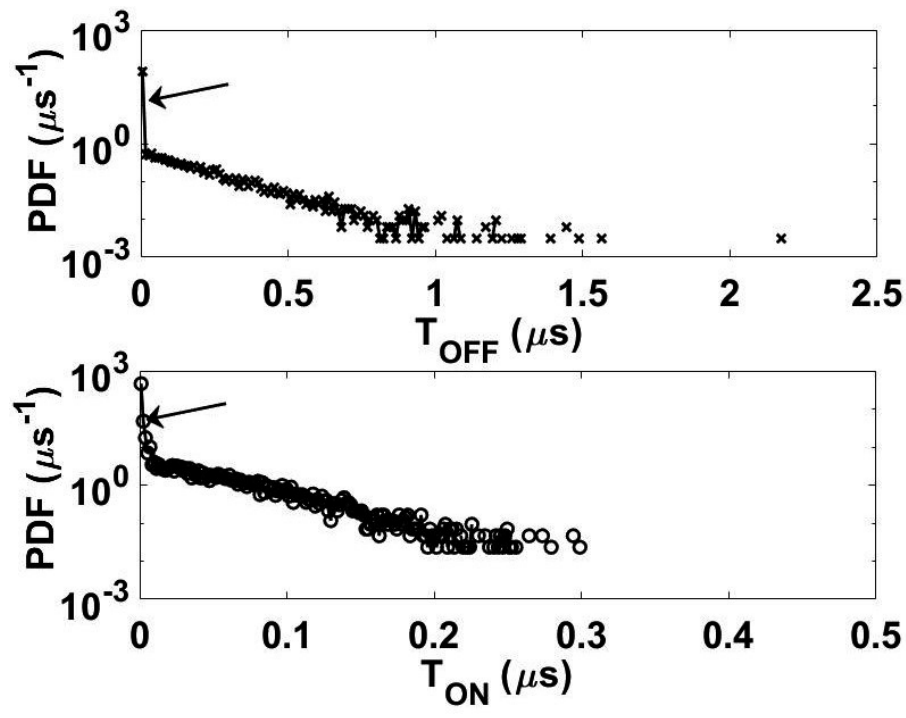

Figure 2.5: The PDF of the inter-arrival times for the ON and OFF states at $14.88 \mathrm{~V}$; the PDFs have different $\mathrm{x}$-axis scales. The arrows indicate where "afterpulsing" plays a role.

\subsubsection{Expected Lifetimes}

Using PDFs as shown in Fig. 2.5, the expected lifetime in each state can now be estimated:

$$
\begin{gathered}
\mathrm{E}\left(T_{\mathrm{ON}}\right)=\sum_{T_{\mathrm{ON}_{\mathrm{i}}}} T_{\mathrm{ON}_{\mathrm{i}}} \cdot \mathrm{P}\left(T_{\mathrm{ON}}=T_{\mathrm{ON}_{\mathrm{i}}}\right) \\
\mathrm{E}\left(T_{\mathrm{OFF}}\right)=\sum_{T_{\mathrm{OFF}_{\mathrm{i}}}} T_{\mathrm{OFF}_{\mathrm{i}}} \cdot \mathrm{P}\left(T_{\mathrm{OFF}}=T_{\mathrm{OFF}_{\mathrm{i}}}\right)
\end{gathered}
$$

where $i$ is the summation index for various values of $T_{\mathrm{ON}}$ and $T_{\mathrm{OFF}}$. Fig. 2.6 shows these obtained $\mathrm{E}\left(T_{\mathrm{ON}}\right)$ and $\mathrm{E}\left(T_{\mathrm{OFF}}\right)$. Note that $\mathrm{E}\left(T_{\mathrm{ON}}\right)+\mathrm{E}\left(T_{\mathrm{OFF}}\right)$ is the expected pulse IAT which is the reciprocal of the expected RTS pulse repetition rate.

\subsubsection{ON-time fraction and Amplitude}

Using the derived $\mathrm{E}\left(\mathrm{T}_{\mathrm{ON}}\right)$ and $\mathrm{E}\left(\mathrm{T}_{\mathrm{OFF}}\right)$, we define the expected ON-time fraction $\mathrm{E}(D)=\mathrm{E}\left(T_{\mathrm{ON}}\right) /\left(\mathrm{E}\left(T_{\mathrm{ON}}\right)+\mathrm{E}\left(T_{\mathrm{OFF}}\right)\right)$. In other words, $\mathrm{E}(D)$ is the fraction of any observation time window where the RTS is in the ON-state. The RTS amplitude $A$ has been defined in section 2.3. Fig. 2.7 shows both $\mathrm{E}(D)$ and $A$ as a function of $V_{\mathrm{R}}$ where $\mathrm{E}(D)$ is shown on a logarithmic scale. 


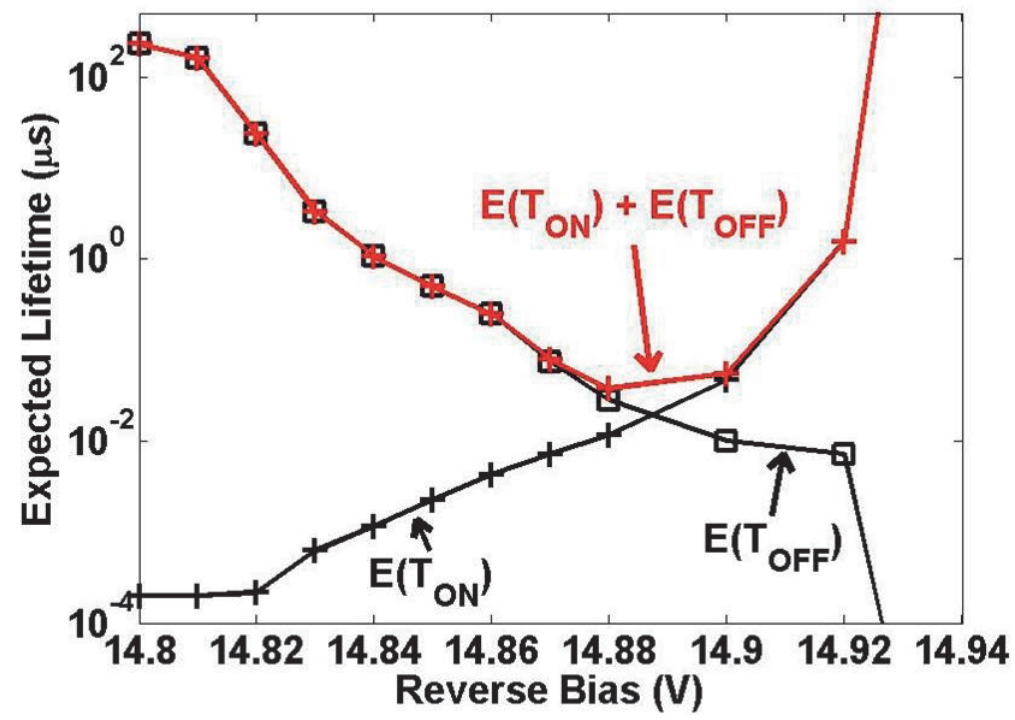

Figure 2.6: Expected lifetimes (in ON and OFF states) and their sum as a function of $V_{\mathrm{R}}$. For $V_{\mathrm{R}}>14.92 \mathrm{~V}$, the avalanche is self-sustaining.

\subsubsection{DC $I-V$ characteristics}

The DC $I-V$ characteristics has been shown in Fig. 2.2. In Fig. 2.3, it was shown that the $I_{R}$ shows RTS behavior in the steep part of the $I-V$ characteristics. The statistical properties of the RTSs were analyzed

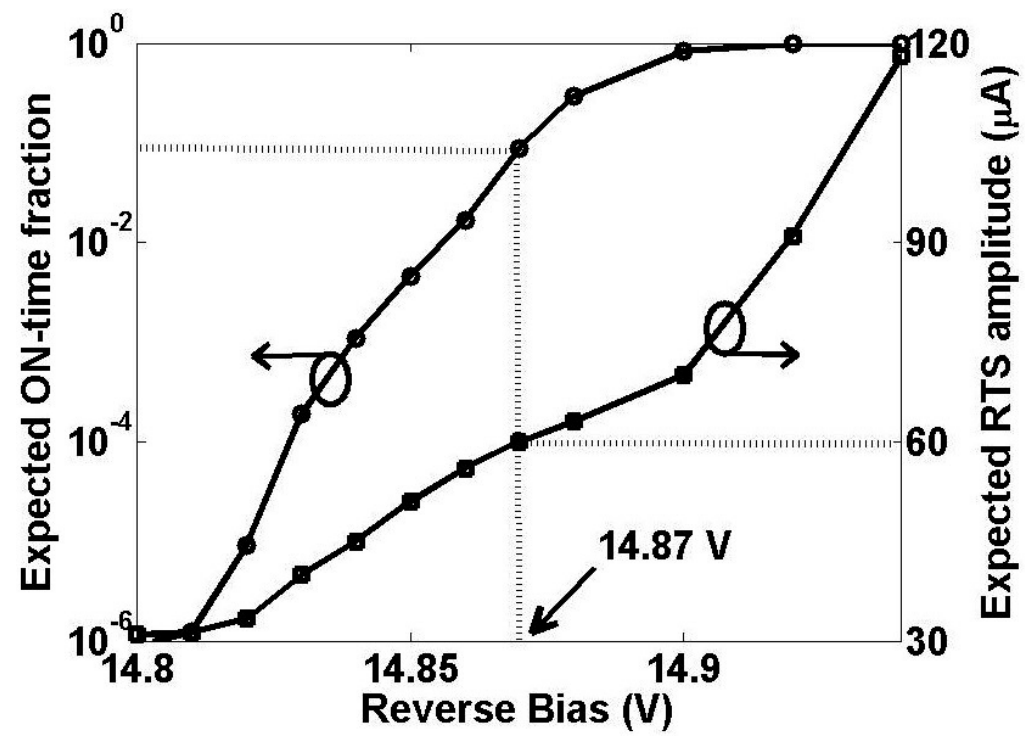

Figure 2.7: Expected $\mathrm{ON}$-time fraction $\mathrm{E}(D)$ and amplitude $A$ as a function of $V_{\mathrm{R}} ; \mathrm{E}(D)$ is on a semi-log scale because of its large dynamic range. The dashed lines explain the design of QRCs in sec. 2.5. 


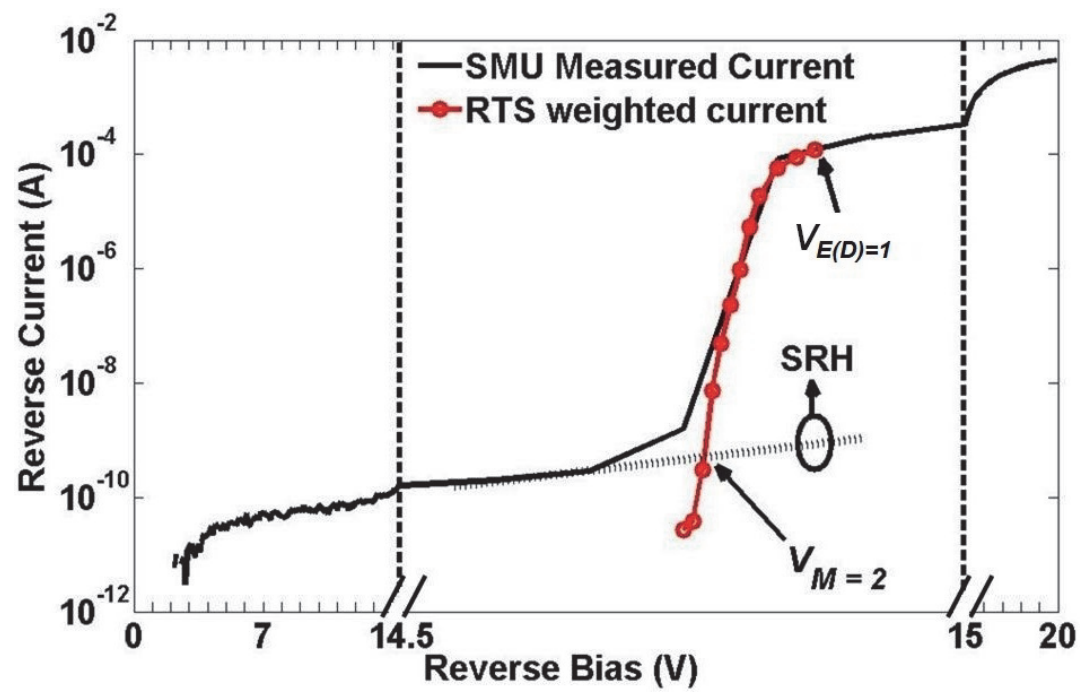

Figure 2.8: DC-measured $I-V$-curve and RTS weighted $\mathrm{E}(D) \cdot A-V$ curve. For clarity, the $\mathrm{x}$-axis has been stretched around the avalanche region. Both $V_{M=2} \approx 14.83 \mathrm{~V}$ and $V_{E(D)=1} \approx 14.92 \mathrm{~V}$ can be used as experimental definitions of $V_{\text {BR }}$.

in sections 2.4.2 and 2.4.3. Using these results, an $\mathrm{E}(D)$-weighted RTS amplitude is derived as $I_{\mathrm{RTS}}\left(V_{\mathrm{R}}\right)=\mathrm{E}(D) \cdot A$. This $I_{\mathrm{RTS}}\left(V_{\mathrm{R}}\right)$ is the DC content of the RTS pulses in $I_{\mathrm{R}}$. Fig. 2.8 shows both DC $I-V$ and $I_{\mathrm{RTS}}\left(V_{\mathrm{R}}\right)$, showing a very good agreement. This implies that the DC $I-V$ characteristics can be explained by the underlying RTS phenomena. Moreover, the steep part of the $I-V$ characteristics is determined by the strong bias dependency of $\mathrm{E}(D)$.

As discussed in section 2.1, experimentally $V_{\mathrm{BR}}$ is not well defined in the literature. We use the statistics of the RTS phenomena to provide a unique definition of two voltages that delimit the steep part of the $I-V$ characteristics. At the beginning of avalanche, the $V_{\mathrm{R}}$ at which the impact ionization contribution equals the Shockley-Read-Hall (SRH) contribution will be denoted as $V_{M=2}$; indicating the voltage at which the multiplication factor $M=2$. The steep part of the $I-V$ characteristics is upper limited at $E(D)=1$ at $V_{\mathrm{R}}=V_{E(D)=1}$. Both voltage levels are indicated in Fig. 2.8. In these devices, the $V_{M=2} \approx 14.83 \mathrm{~V}$ and $V_{E(D)=1} \approx 14.92 \mathrm{~V}$. Both these values can be used for an experimental definition of $V_{\mathrm{BR}}$.

\subsection{Applications}

\subsubsection{Application to SPADs}

A possible application of the RTS analysis could be in the design of passively quenched SPAD QRCs. In those circuits, the quenching time $\left(\tau_{\mathrm{Q}}\right)$ 
is usually much shorter than the recharge time $\left(\tau_{R}\right)$ [88]. The $\tau_{R}=C_{P} R_{Q}$ limits the count rate in SPADs, where $C_{\mathrm{P}}$ is the total capacitance (parasitic capacitance plus the intrinsic capacitance of the SPAD) at the quenching terminal of the SPAD and $R_{\mathrm{Q}}$ is the external quenching resistance. An ill-defined value of $R_{\mathrm{Q}}$ can result in a lower maximum count rate from SPADs. In addition, a higher $R_{\mathrm{Q}}$ results in a larger area and a larger $C_{\mathrm{P}}$. The latter results in a larger amount of charge per avalanche event $\left(Q_{\mathrm{AVAL}}\right)$ and hence in higher afterpulsing [88].

$R_{\mathrm{Q}}$ can be accurately calculated for SPADs in any technology using the statistical properties of the RTSs. For example, in this $140 \mathrm{~nm}$ technology, for an $5 \% \mathrm{E}(D)$ (or $95 \%$ probability of quenching), at these quench conditions, $V_{\mathrm{R}}=14.87 \mathrm{~V}$ and $\mathrm{A} \approx 60 \mu \mathrm{A}$ (Fig. 2.7). If the final operating voltage $\left(V_{\mathrm{f}}\right)$ in a passive $\mathrm{QRC}$ is set to $14.87 \mathrm{~V}, R_{\mathrm{Q}}$ can be calculated using [22]:

$$
R_{\mathrm{Q}}=\frac{V_{\mathrm{R}}-V_{\mathrm{f}}}{60 \mu \mathrm{A}}
$$

Therefore, $R_{\mathrm{Q}}$ should be $\approx 16 \mathrm{k} \Omega$ per extra volt of $V_{\mathrm{R}}-V_{\mathrm{f}}$ (assuming $R_{\mathrm{Q}}>>$ resistance of the diode). $V_{\mathrm{R}}-V_{\mathrm{f}}$ can be interpreted as the ill-defined $V_{\mathrm{EX}}$ in the literature $[22,66,88]$. The traditional rule-of-thumb would suggest to use an $R_{\mathrm{Q}}=50 \mathrm{k} \Omega$ [22], while using the conventional DC $I-V$ characteristics, $R_{\mathrm{Q}} \geqslant 100 \mathrm{k} \Omega$ per volt of $V_{\mathrm{R}}-V_{\mathrm{f}}$. By optimizing the $R_{\mathrm{Q}}$, the count rate can be increased by a factor of 3 to 6 compared to traditional approaches. A parametric self-sustaining avalanche current $\left(I_{\text {lat }, p}\right)$ can be defined with $\mathrm{E}(D)$ (or probability of quenching) as the main parameter.

To explicitly show the advantage of the proposed method in reducing $Q_{\text {AVAL }}$ in integrated QRCs, a circuit simulation was done in a $140 \mathrm{~nm}$ SOI CMOS technology [36] to estimate $Q_{\mathrm{AVAL}}$. The simulation setup is shown in Fig. 2.9 (inset). A verilog-A simulation model for the SPAD was used [89]. The turnoff probability was taken from experimental data (Fig. 2.7) instead of using the rule-of-thumb of $I_{\text {lat }}=100 \mu \mathrm{A}$ [22]. The $Q_{\mathrm{AVAL}}=$ $\int_{0}^{T_{\text {AVAL }}} I_{\mathrm{AVAL}}(\mathrm{t}) \mathrm{dt}$, where $I_{\mathrm{AVAL}}(\mathrm{t})$ is the current during the avalanche event and $T_{\mathrm{AVAL}}$ is the duration of the avalanche event. Fig. 2.9 shows that at higher $V_{\mathrm{R}}-V_{\mathrm{f}}, Q_{\mathrm{AVAL}}$ can be significantly reduced using the proposed method for estimating $R_{\mathrm{Q}}$ because of the lower $C_{\mathrm{P}}$. A minimal $Q_{\mathrm{AVAL}}$ reduces the undesired afterpulsing phenomena in addition to reducing the energy consumption.

\subsubsection{Application to Avalanche Mode LEDs}

As mentioned earlier, Si avalanche mode LEDs (AMLEDs) are attractive for monolithic integration of optical links in CMOS technologies [14]-[76].

For data communication using large signal modulation schemes (e.g. On-Off Keying) with AMLEDs [71], the randomness of the avalanche current should be considered. It is then important to have AMLEDs with a relatively high number of defects to reduce jitter and the bit-error-rate in 


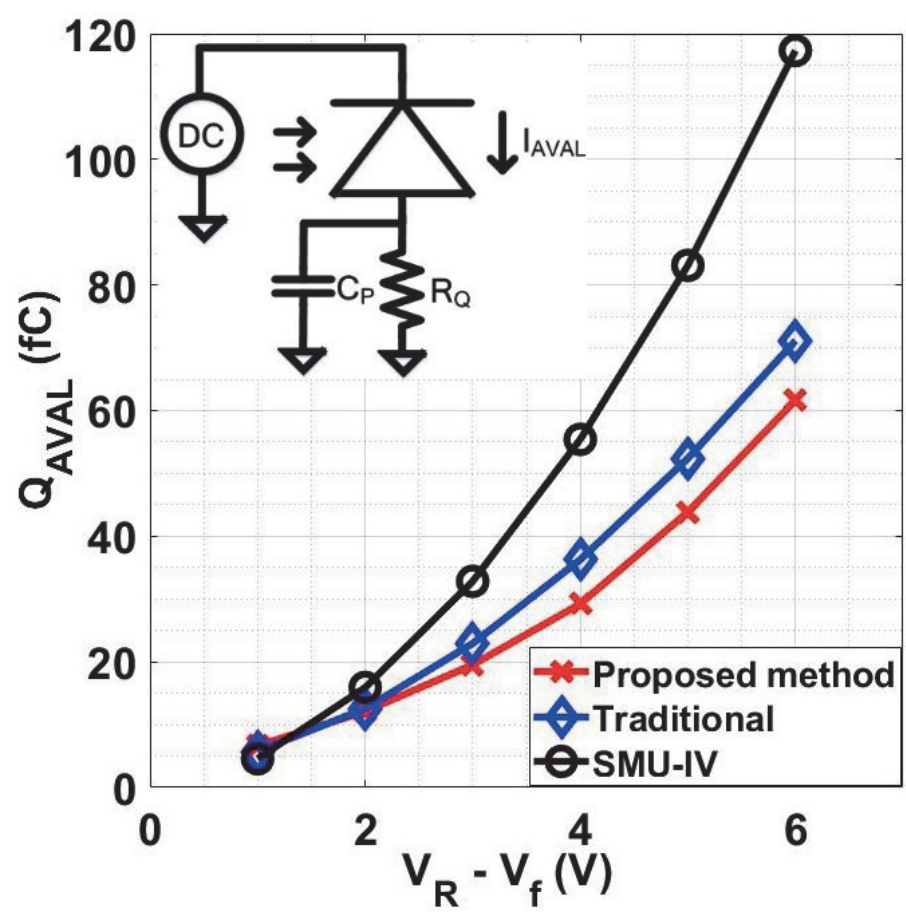

Figure 2.9: Simulated avalanche charge per event $\left(Q_{\mathrm{AVAL}}\right)$ as a function of $V_{\mathrm{R}}-V_{\mathrm{f}}$ using all three methods of estimating $R_{\mathrm{Q}}$ (proposed $16 \mathrm{k} \Omega$ per $1 \mathrm{~V}$ of $V_{\mathrm{R}}-V_{\mathrm{f}}$, traditional rule-of-thumb of $50 \mathrm{k} \Omega$ per $1 \mathrm{~V}$ of $V_{\mathrm{R}}-V_{\mathrm{f}}$ and SMU $I-V$ based $100 \mathrm{k} \Omega$ per $1 \mathrm{~V}$ of $V_{\mathrm{R}}-V_{\mathrm{f}}$ ). Inset shows the simulation setup.

the communication link, which is actually exactly the opposite requirement for SPADs. This is discussed in Chapter 4.

\subsection{Conclusions}

RTS phenomena in diode currents were shown to fully determine the steep current-voltage $(I-V)$ dependency in avalanche. Using a time domain analysis, we determined the statistical properties of these RTS phenomena. The statistical properties were shown to fully describe the steep part of the $I-V$ characteristics. An accurate experimental definition of breakdown voltage $\left(V_{\mathrm{BR}}\right)$ was proposed based on the RTS analysis. Using the RTS analysis, the value of the self-sustaining avalanche current in diodes was parametrically determined, that can be used in an accurate design of quenching and recharge circuits (QRCs) for SPADs. It is expected that the proposed approach can significantly increase count rates while reducing afterpulsing. The advantages of the accurate design of $\mathrm{QRC}$ s were discussed with circuit simulations. 


\title{
RTS PHENOMENA IN ULTRA-SHALLOW SILICON AVALANCHE DIODES
}

\begin{abstract}
An extensive time domain analysis of the Random Telegraph Signal (RTS) phenomena in ultra-shallow silicon avalanche diodes is presented. Experiments show two distinct types of RTSs classified herein, on the basis of the temporal behavior of the amplitude, as the "decaying" and the "constant" type. These RTSs are analyzed using a model for defects reported earlier, from which their ohmic series resistance and geometrical parameters have been estimated. The results indicate that breakdown of a relatively small area defect results in a "decaying" amplitude type of RTS, and breakdown of a relatively large area defect results in a "constant" amplitude type of RTS. These two types can be explained by the differences in the thermal resistance, which is higher for the former.
\end{abstract}




\subsection{Introduction}

Deterministic and statistical carrier multiplication theories have been reported in literature to describe the triggering of avalanche in silicon (Si) diodes in [25, 73, 74, 90] (and in references therein). In applications like optical detectors based on avalanche photodiodes (APDs) [9] or single-photon avalanche diodes (SPADs) [75], the avalanche phenomenon is utilized to detect weak optical signals. Moreover, during avalanche, Si diodes emit light at visible wavelengths, which is attractive for monolithic integration of optical links in CMOS technologies because of significant overlap of their emission spectrum with the responsivity of standard Si photodetectors $[14,16,41,76]$.

Random Telegraph Signal (RTS) phenomena in the avalanche current at the onset of breakdown were reported earlier [42, 43]. Initially, the RTS phenomena were referred to as the "microplasma instability" because during breakdown, it was shown that these unstable localized defects emitted visible light [46]. Many interesting theories were reported to provide a phenomenological description of these current fluctuations $[45,48,49,52]$. It was established that these fluctuations arise from crystal defects such as dislocations in the diodes [49]. The concept of RTS phenomena to model these fluctuations was discussed in [45]. Recently, the modeling has been revisited [74] and an elaborate overview of the evolution of this topic has also been presented in the same paper [74].

In chapter 2 , it is discussed that the avalanche process and its currentvoltage $(I-V)$ characteristics can be described by RTS phenomena. Using the RTS analysis results, the $I-V$ characteristics could be modeled. The impact of the RTS analysis on the accurate design of quenching and recharge circuits for SPADs is also discussed in chapter 2. That work can be used to increase the count rates and to decrease the afterpulsing in SPADs. Further, the non-monotonic behavior of the noise spectral density in reverse biased diodes is caused by RTS phenomena [91]. As discussed in [74, 92], studying RTS phenomena is also useful for determining the material quality and for reliability analyses of e.g. power devices, because RTS phenomena are caused by crystal defects.

Time domain analysis (TDA) of the RTS phenomena can be used to estimate the properties of defects causing these RTSs. The TDA methods and the experimental setup to characterize RTSs were developed after 1960s. Possibly because of experimental limitations, an extensive TDA of RTS phenomena in avalanche diodes is missing in the literature. The purpose of this chapter is to address this issue. The main findings of this chapter are:

- From the temporal behavior of the RTS amplitude, it is shown that two types of defects with different local thermal impedances exist in diodes. Defects with a high thermal impedance cause a "decaying" 
a)

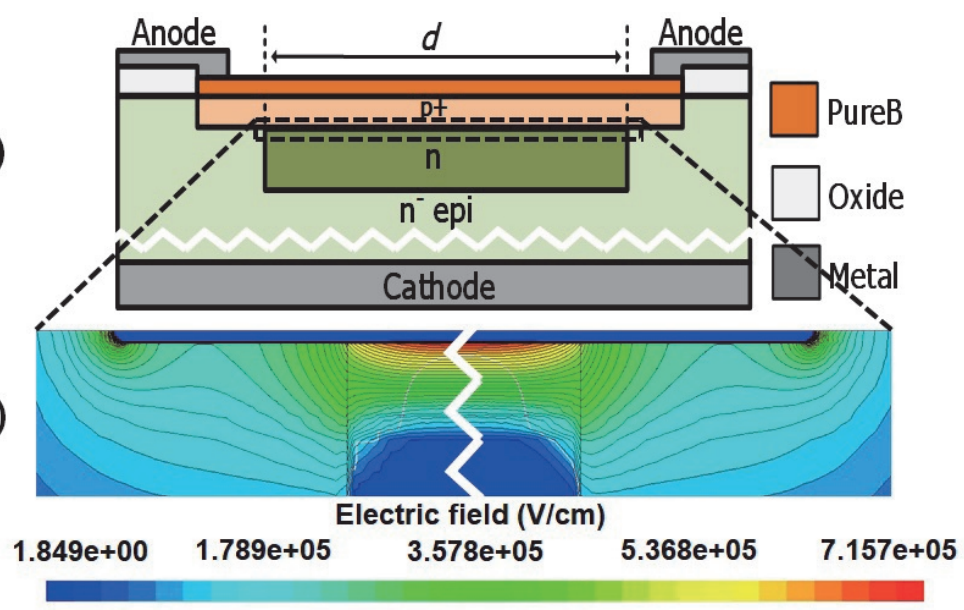

Figure 3.1: (a) Schematic cross-section of a circular $\mathrm{p}^{+} \mathrm{n}$ diode in PureB technology. The diameter is defined by the dimensions of the $n$ layer. (b) TCAD simulated electric field of the highlighted region above breakdown $(15 \mathrm{~V})$ showing a lateral uniform field in the depletion region.

type of amplitude in RTSs and defects with a low thermal impedance cause a "constant" type of amplitude in RTSs.

- From the TDA, it is shown that the bumpy behavior in the currentvoltage characteristics is caused by the two types of defects and not necessarily by the relatively high thermal impedance of the diode packaging as reported earlier [49].

- An existing model for these defects is improved to take into account an explicit thermal model. Using the model, both the ohmic series resistance and the dimensions of these defects are estimated.

The chapter is outlined as follows: the experimental diodes and measurement setup to measure the RTS phenomena are described in section 3.2. The analysis method for the two types of RTSs is discussed in section 3.3. In section 3.4, the geometry dependency of various RTS parameters is shown. A model for the defects causing these RTS phenomena is used for estimating some of the electrical and geometrical parameters of the defects in section 3.5. Finally, in section 3.6 the main findings of this chapter are summarized.

\subsection{Experimental setup}

\subsubsection{Experimental diodes}

In this chapter, the aim is to study RTS phenomena in the avalanche processes in devices used for optical generation and detection, requiring semiconductor-only junctions. Therefore, for the experiments, diodes were 


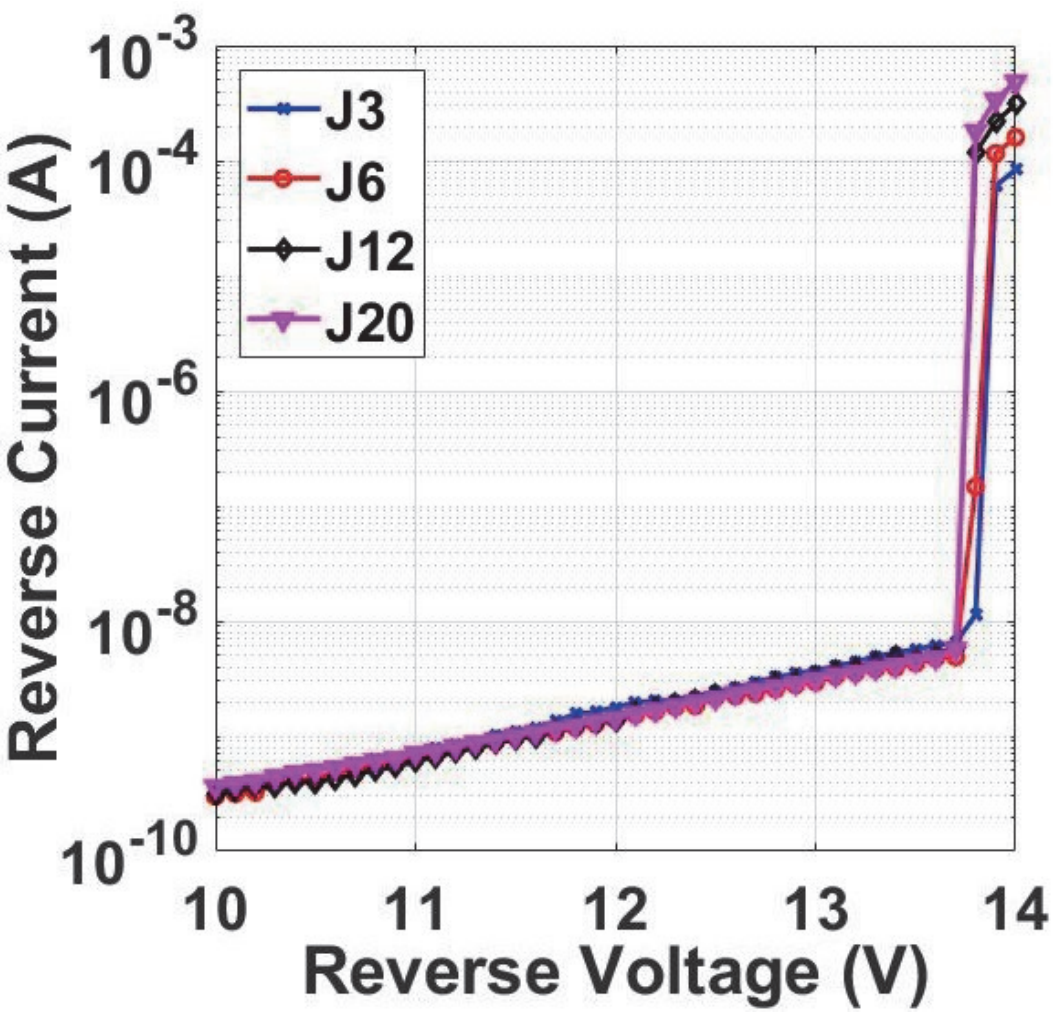

Figure 3.2: Measured reverse $I-V$ characteristics of all diodes at $25^{\circ} \mathrm{C}$.

designed in a pure boron (PureB) technology [93]. In this technology, a thin layer of PureB is deposited by chemical vapor deposition on a clean n-Si surface. P-type $S i$ is obtained from this thin PureB layer and high quality ultra-shallow $\mathrm{p}^{+}$-n, hence abrupt asymmetric, junctions are obtained [94]. These ultra-shallow junctions are suitable for various optical detection or emission applications [93-95]. Four circular diodes, on the same die, were selected with diameters of $3 \mu \mathrm{m}, 6 \mu \mathrm{m}, 12 \mu \mathrm{m}$ and $20 \mu \mathrm{m}$; these diodes are labeled as J3, J6, J12 and J20 respectively, where the diode name indicates its diameter. A TCAD simulated electric field of a representative device at breakdown showing a uniform lateral field in the depletion region is shown in Fig. 3.1(b). Due to the circular geometry, the lateral electric field in the depletion region should be uniform at breakdown. However, the electric field could be distorted at crystal defects, forming the preferred location of breakdown [49].

Fig. 3.2 shows DC $I-V$ characteristics of all diodes at $25^{\circ} \mathrm{C}$ in dark conditions measured by an Agilent B2901A Source/Measure Unit (SMU) using $1 \mathrm{~s}$ integration time. In these diodes, the avalanche starts at around 13.7 V; the reverse current $\left(I_{R}\right)$ rises sharply between the reverse voltage $\left(V_{\mathrm{R}}\right)$ of 13.7 and $13.9 \mathrm{~V}$. This chapter focuses on this voltage range. 

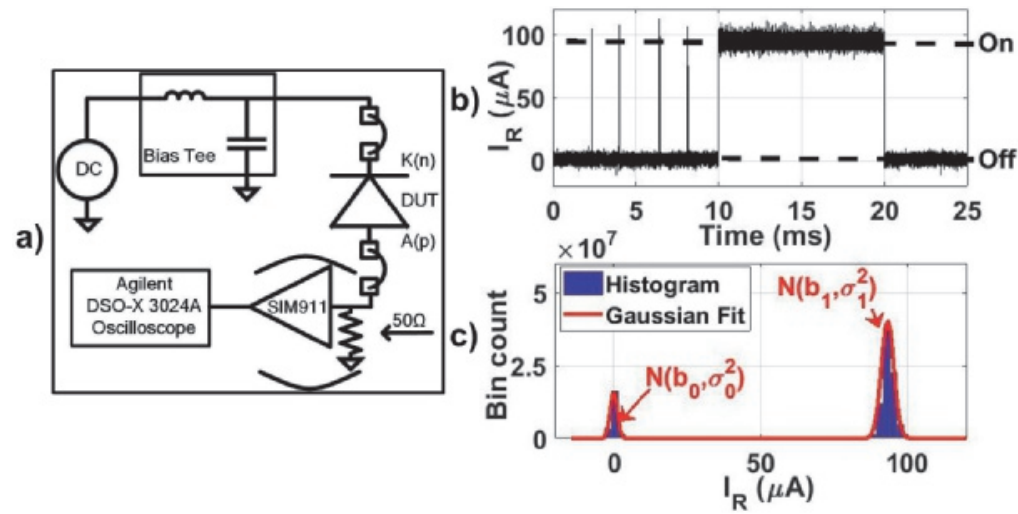

Figure 3.3: (a) RTS measurement setup. (b) Example of measured RTSs in $I_{R}$. (c) Histogram of the measured RTSs.

\subsubsection{Measurement setup and analysis method}

The measurement setup and the TDA method have been described extensively in chapter 2 . The relevant details are briefly summarized here.

Fig. 3.3(a) shows the experimental setup to measure the RTS phenomena. The cathode is biased at a constant voltage and the RTSs were measured across a $50 \Omega$ resistance, thus providing a low impedance load and a low quenching. A low noise SIM 911 preamplifier was used to drive the oscilloscope input to improve the signal-to-noise ratio at the oscilloscope input. The transient data were acquired for a total duration of $1 \mathrm{~s}$ at each reverse bias voltage for all diodes, at a data acquisition rate of 100 MS/s. The measurements were done in a Faraday cage in dark condition using wafer probing.

Fig. 3.3(b) shows an example of the measured $I_{\mathrm{R}}$ in the steep part of the $I-V$ characteristics, showing the RTS phenomena in $I_{\mathrm{R}}$ in these diodes. This $I_{\mathrm{R}}$ can be represented in a histogram as shown in Fig. 3.3(c). A sum of two Gaussians, $\mathrm{N}\left(b_{0}, \sigma_{0}^{2}\right)$ and $\mathrm{N}\left(b_{1}, \sigma_{1}^{2}\right)$, is fitted on this histogram and from the fit parameters, the mean value of the off-state $\left(b_{0}\right)$ and the mean value of the on-state $\left(b_{1}\right)$ are estimated. The RTS amplitude $(A)$ is obtained from these fit parameters. Also, from the mean on-time $\left(\mathrm{E}\left(T_{\mathrm{ON}}\right)\right)$ and mean off-time $\left(\mathrm{E}\left(T_{\mathrm{OFF}}\right)\right)$, the mean on-time fraction $(\mathrm{E}(D))$ can be estimated, the detailed procedure has been described in chapter 2. The mathematical equations for the estimation of relevant parameters are summarized in Eq. (3.1):

$$
\begin{gathered}
A=b_{1}-b_{0} \\
\mathrm{E}(D)=\frac{\mathrm{E}\left(T_{\mathrm{ON}}\right)}{\mathrm{E}\left(T_{\mathrm{ON}}\right)+\mathrm{E}\left(T_{\mathrm{OFF}}\right)} .
\end{gathered}
$$

$E(D)$ represents the fraction of time, in an observed time window, where the RTS is in the on-state (Fig. 3.3(b)). Note that two types of RTSs are ob- 

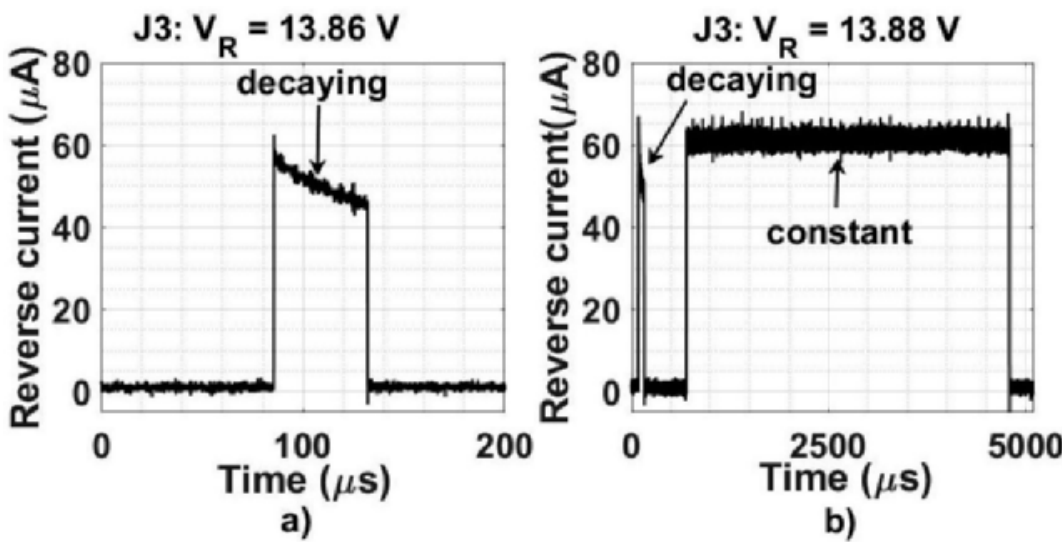

Figure 3.4: (a) A "decaying" type of RTS observed at a lower $V_{\mathrm{R}}$. (b) Mixture of a "constant" and a "decaying" RTS at a higher $V_{\mathrm{R}}$ in the same diode. The $\mathrm{x}$-axis scales are different for clarity.

served in these diodes (see section 3.3). In the definitions of $\mathrm{E}(D)$ and $A$, no distinction between these two types of RTSs has been made. Multiplication noise of the diodes $\left(=\sqrt{\sigma_{1}^{2}-\sigma_{0}^{2}}\right)$ is disussed in appendix A.

\subsection{RTS characterization}

As mentioned above, two types of RTSs have been observed in these diodes. For example, for J3, at a relatively low $V_{\mathrm{R}}$ (at the onset of breakdown), the RTSs were decaying in amplitude during RTS events as shown in Fig. 3.4(a). These are denoted as the "decaying" amplitude type of RTSs. At a higher $V_{\mathrm{R}}$ (slightly above the breakdown voltage), another type of RTS with a constant amplitude, denoted as the "constant" amplitude type was also observed, as shown in Fig. 3.4(b). To our best knowledge, a mixture of "decaying" and "constant" types of RTSs at a given bias in a single diode has not been reported before.

A possible explanation for the two types of the RTSs was provided in [49]. It was reported that the "decaying" RTSs will be observed in diodes which do not have a good thermal contact with the outside world. For such diodes, the energy dissipated during the avalanche process would increase the temperature of the diode. This decreases the effective impact ionization coefficient $(\alpha)$ and consequently decreases the avalanche current [80]. Interestingly, it was assumed that the local thermal impedance of a defect inside the diode is negligible [49]. The above explanation implies that a mixture of defects with different local thermal impedances in a single device is not possible, contrary to the observations. Assuming thermal effects as the root cause for the different RTSs would imply that there are at least two different defects in the same diode: one with a relatively low and the other with a relatively high thermal impedance with the outside world. 


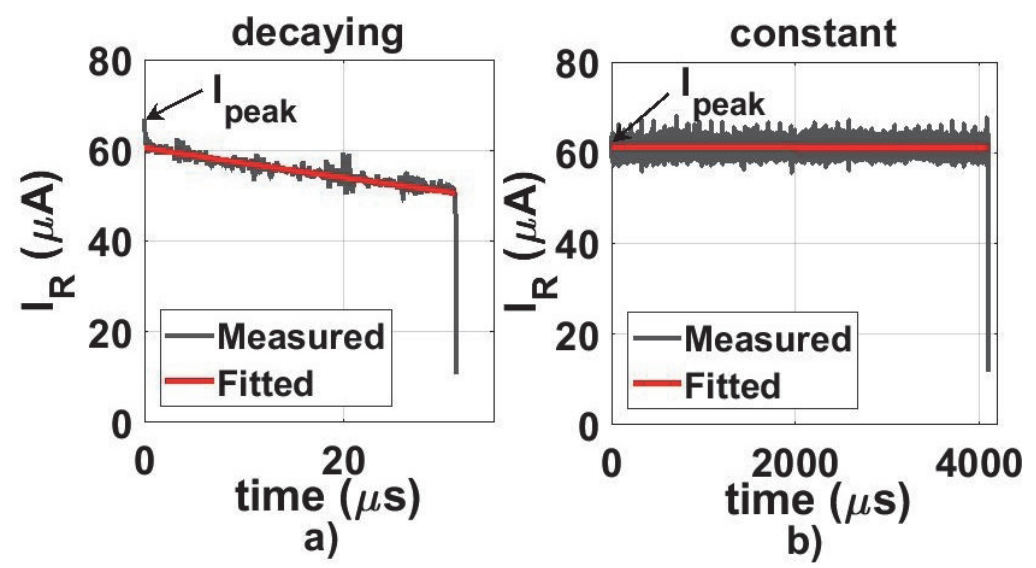

Figure 3.5: Classifying the RTS: (a) A "decaying" RTS : $P W=31.47 \mu \mathrm{s}, \tau=$ $183.9 \mu \mathrm{s}$, and a decay of $\sim 16 \%$. (b) A "constant" RTS : PW $=4.09 \times 10^{3} \mu \mathrm{s}$, $\tau=3.3 \times 10^{6} \mu \mathrm{s}$. The peak current $\left(I_{\text {peak }}\right)$ was separately extracted.

\subsubsection{RTS classification}

The measured transient data were analyzed in Matlab. From the transient data acquired for $1 \mathrm{~s}$, all individual RTSs were identified and many RTS properties were extracted. These properties are the peak current $\left(I_{\text {peak }}\right)$, pulse width $(P W)$, the decay time constant for the "decaying" RTSs $(\tau$, see section 3.5) and the shape of the RTS.

To distinguish between the "decaying" and the "constant" types of RTS, the decay in the RTS amplitude during each pulse was estimated. Firstly, the $P W$ of each RTS was extracted from its rising and falling edges. For estimating the time constant $(\tau)$, the RTS current $I_{\mathrm{R}}(t)$ was fitted onto an exponentially decaying transient current model (Fig. 3.5):

$$
I_{R}(t)=I_{0} \cdot e^{-t / \tau},
$$

where $\tau$ is a fitting parameter. Based on the ratio of $P W / \tau$, the decay in the RTS amplitude was calculated. For a decay of less than $1 \%$ during the $P W$, the shape of the RTS was labeled as a "constant" amplitude type.

$$
\text { RTS type }= \begin{cases}\text { constant amplitude, } & \text { if } \frac{P W}{\tau} \leqslant 0.01 \\ \text { decaying amplitude } & \text { otherwise }\end{cases}
$$

Fig. 3.5 shows an example of a measured and its fitted pulse for both types of RTSs.

The peak current ( $I_{\text {peak }}$, see Fig. 3.5) of each RTS was also separately extracted. The $I_{\text {peak }}$ is used in section 3.5 to estimate the parameters of the defects. 


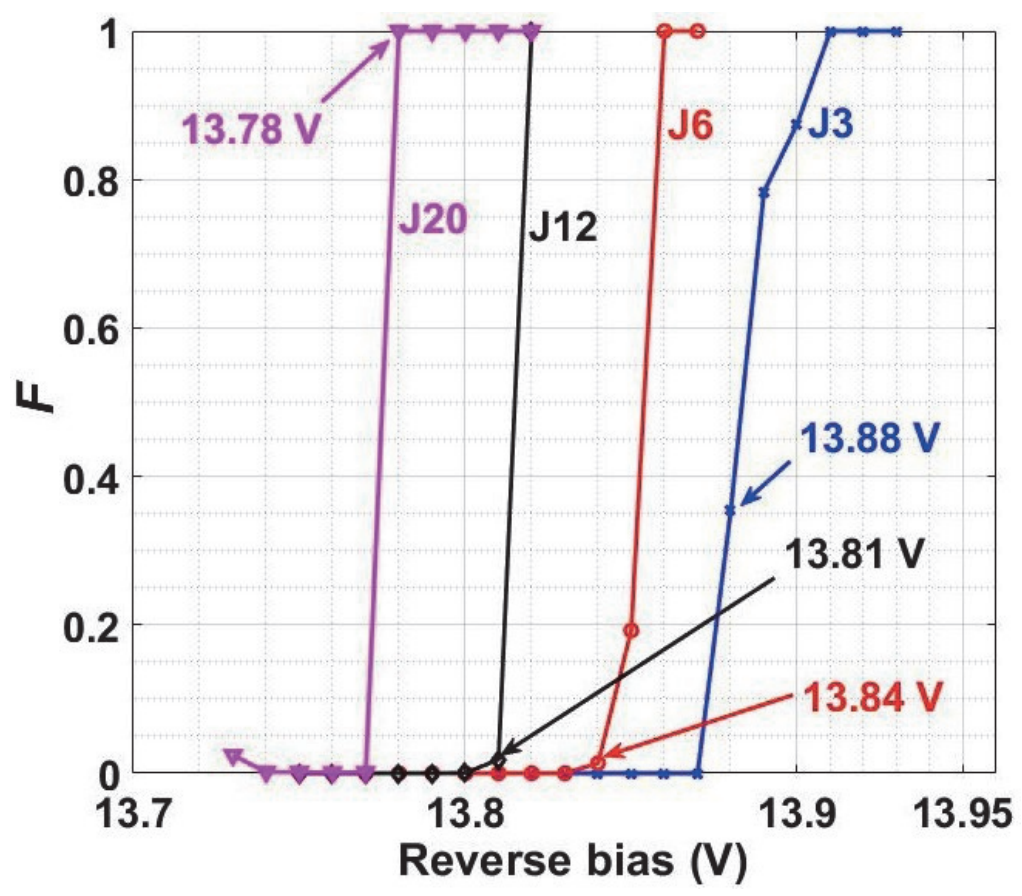

Figure 3.6: Fraction of "constant" amplitude RTSs $(F)$ as a function of $V_{\mathrm{R}}$.

\subsubsection{Fraction of "constant" RTSs}

Fig. 3.6 shows the fraction of "constant" type of RTSs $\left(F\left(V_{\mathrm{R}}\right)\right)$ as a function of $V_{\mathrm{R}}$ for all diodes:

$$
F\left(V_{\mathrm{R}}\right)=\frac{N_{\text {constant }}\left(V_{\mathrm{R}}\right)}{N_{\text {constant }}\left(V_{\mathrm{R}}\right)+N_{\text {decaying }}\left(V_{\mathrm{R}}\right)},
$$

where $N_{\text {constant }}\left(V_{\mathrm{R}}\right)$ and $N_{\text {decaying }}\left(V_{\mathrm{R}}\right)$ are the number of "constant" and "decaying" types of RTSs per unit time respectively, observed at each $V_{\mathrm{R}}$. From the $F-V$ curve, the $V_{\mathrm{R}}$ at which the "constant" type of RTSs start appearing can be estimated, i.e. $F>0$. At a few bias points, $0<F<1$, both types of RTSs were observed.

Fig. 3.6 shows that the "constant" type of RTSs appear for higher $V_{\mathrm{R}}$ than the "decaying" type of RTSs. As discussed in section 3.4, the $V_{\mathrm{R}}$ at which $F$ starts to sharply increase is the $V_{\mathrm{R}}$ at which a bump in the RTS parameters, $A$ and $\mathrm{E}(D)$, were observed.

\subsubsection{Mean Pulse width}

Fig. 3.7 shows the mean pulse width $\left(P W_{\text {MEAN }}\right)$ of the "constant" and the "decaying" RTSs as a function of $V_{R}$. The mean values were obtained from all observed RTSs for each type at each bias condition. The "decaying" RTSs have a lower $P W_{\text {MEAN }}$ compared to the "constant" RTSs. This can be explained by the local heating providing a negative feedback for the 


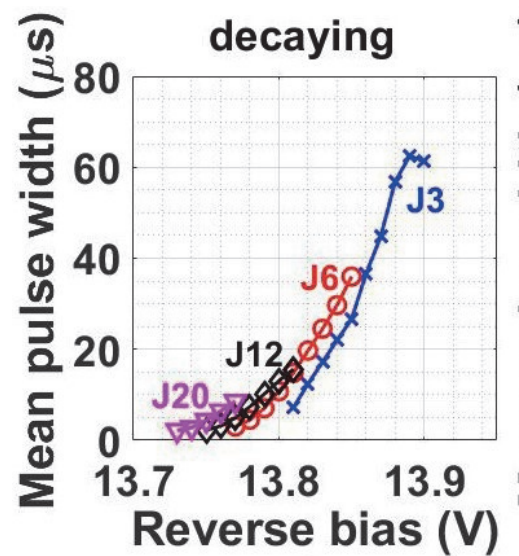

a)

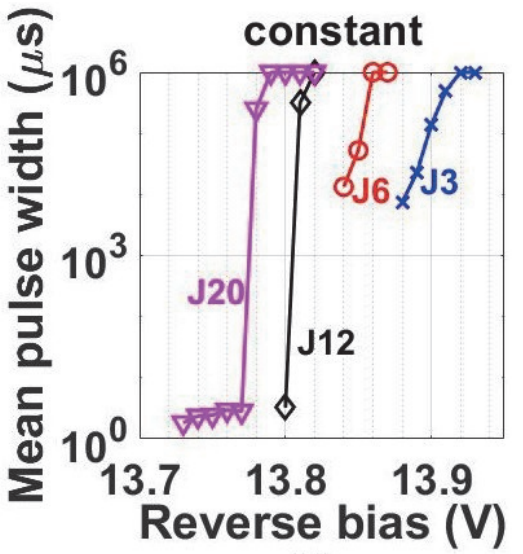

b)

Figure 3.7: Mean pulse width $\left(P W_{\text {MEAN }}\right)$ as a function of $V_{\mathrm{R}}$ for (a) "decaying" (b) "constant" amplitude RTSs. Y-axis are different for clarity. A $P W_{\text {MEAN }}$ of $1 \mathrm{~s}$ implies that no RTSs were observed at that bias setting and the diode was in a continuous On-state.

avalanche process by reducing the excess bias across the diode (section 3.5), thereby reducing $\alpha$. The avalanche at such a "hotspot" quenches relatively quickly because of a decreasing $\alpha$ [80]. Further, the $P W_{\text {MEAN }}$ increases with $V_{\mathrm{R}}$ because of the increasing electric field, which helps to sustain the avalanche for a longer time. The "constant" amplitude RTSs have much larger $P W_{\text {MEAN }}$, possibly due to the absence of significant thermal effects. For "constant" RTSs, the accuracy of the estimated $P W_{\text {MEAN }}$ reduces for higher $V_{\mathrm{R}}$ because of fewer events in $1 \mathrm{~s}$ measurement time. However, the measured accuracy is sufficient for the purpose of modeling and estimation of the defect parameters.

\subsection{Bias dependent RTS parameters}

The statistical properties of the RTSs, namely $A$ and $\mathrm{E}(D)$ were obtained as a function of $V_{\mathrm{R}}$ for all diodes. The arrival times between the RTS pulses, also referred to as inter-arrival times have been shown to be exponentially distributed $[49,68,96]$, which confirms that the observed RTS process has a Poisson distribution.

\subsubsection{RTS Amplitude}

Fig. 3.8 shows that $A$ increases with $V_{\mathrm{R}}$. The bump in the $A-V$ characteristics at the indicated $V_{\mathrm{R}}$ (Fig. 3.8) is also important to note. Such a bump was believed to be caused by the relatively high thermal impedance of the diode with the outside world [49]. It was thought that if the diode was not in a good thermal contact with its heat sink, such $A-V$ characteris- 


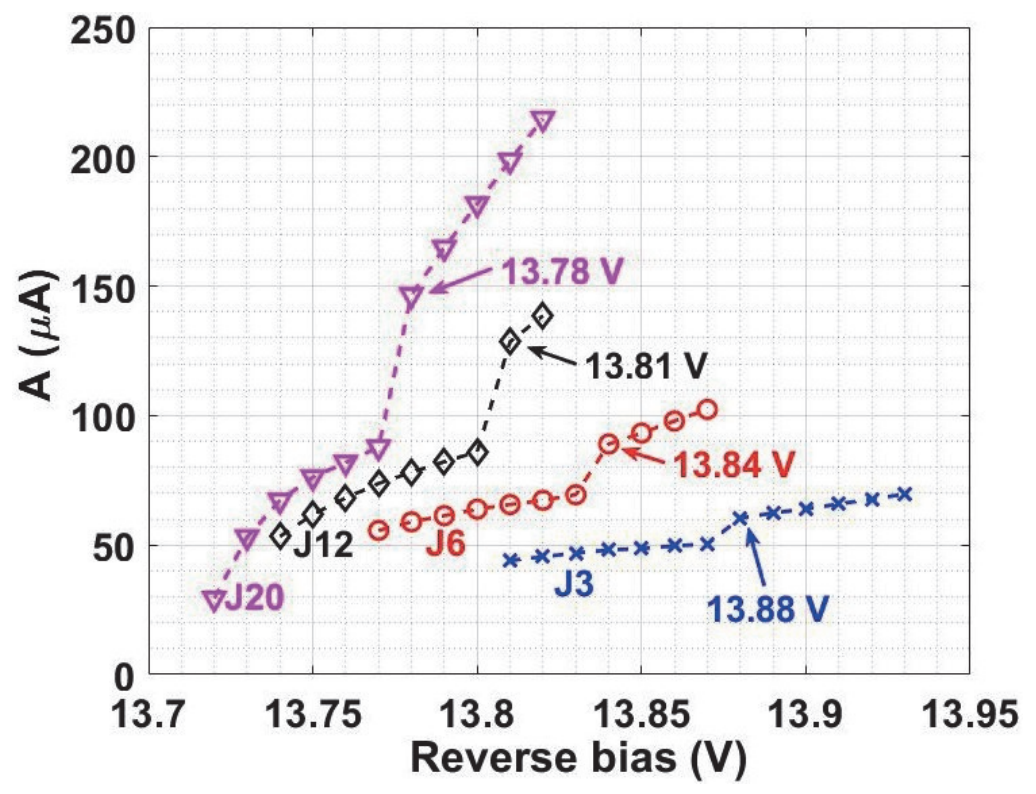

Figure 3.8: RTS amplitude $(A)$ as a function of $V_{\mathrm{R}}$. At the indicated $V_{\mathrm{R}}$, "constant" amplitude RTSs are triggered (Fig. 3.6).

tics would be obtained. However, as shown in section 3.3, at and above these $V_{\mathrm{R}}$, defects causing the "constant" amplitude RTSs are triggered; the bumpy behavior is a result of those RTSs.

\subsubsection{Mean On-time fraction}

Fig. 3.9 shows $E(D)\left(E q\right.$. (3.1b)) as a function of $V_{\mathrm{R}}$. The $E(D)$ sharply increases as a function of $V_{\mathrm{R}}$ close to breakdown. As explained in chapter 2 , the steep $I-V$ dependency in avalanche is mainly because of this steep dependency of $\mathrm{E}(D)$ on $V_{\mathrm{R}}$. The bump at a higher $V_{\mathrm{R}}$ is due to the onset of the "constant" amplitude type of RTSs at higher $V_{\mathrm{R}}$ which have higher $P W_{\text {MEAN }}$, as discussed in section 3.3. As the size increases (from J3 towards $\mathrm{J} 20$ ), the $V_{\mathrm{R}}$ range over which the RTS phenomena are observed (the range in which $0<\mathrm{E}(D)<1$ ) decreases. This can be attributed to an increasing number of crystal defects with increasing size of the diode [97].

The $A-V$ and $\mathrm{E}(D)-V$ characteristics are used to estimate a few properties of defects in section 3.5 .

\subsection{Estimation of model parameters}

In this section, the electrical and geometrical parameters of the defects causing the RTS phenomena are estimated, based on the model from [49]. First, the model and its relevant characteristics are discussed. Then, the electrical parameters of the defects are estimated using TDA results. From 


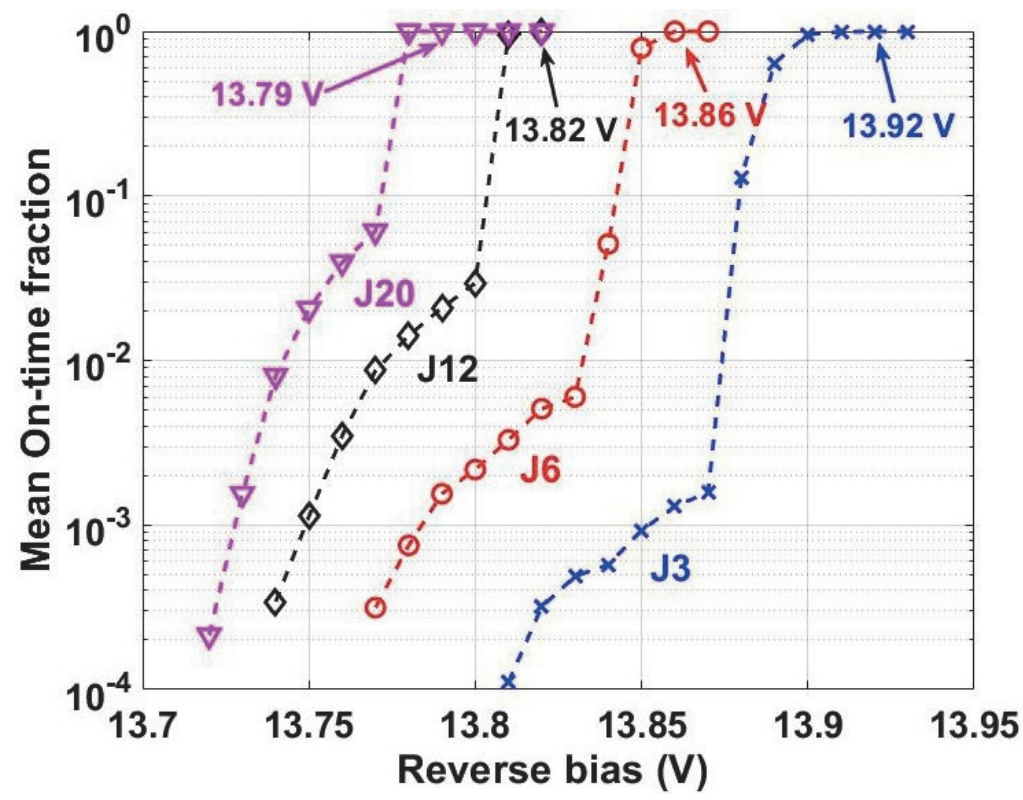

Figure 3.9: Mean $\mathrm{ON}$-time fraction $(\mathrm{E}(D))$ as a function of $V_{\mathrm{R}}$. At and above the indicated $V_{\mathrm{R}}, \mathrm{E}(D)=1$; the diodes were in a continuously On-state above these $V_{R}$.

the electrial parameters, geometrical details of the defects causing the two types of RTSs are estimated.

Fig. 3.10(a) shows the schematic cross-section of a defect causing these RTS phenomena. For simplicity, a defect is modeled as a cylinder of height $w$ and diameter $d$ and avalanche is assumed to be confined in this cylinder [49]. As reported earlier, $w$ is the depletion layer width [49, 74].

Fig. 3.10(b) shows the circuit model of the defect. The bistable switch models the on-off switching characteristics of the defect causing the RTS phenomenon. The charge fluctuations in the defect region control this switch [74]. The $V_{\mathrm{a}}(\mathrm{t})$ in the model corresponds to the voltage at which the multiplication factor $(M)$ is unity [49]. Once the avalanche is triggered, the free carriers partially neutralize the space charge and thereby reduce the electric field. Then, the applied $V_{\mathrm{R}}$ should be increased to keep $I_{\mathrm{R}}$ constant; the resistance produced by this partial neutralization of space charge because of high injection in the defect region is denoted as the space charge resistance $\left(R_{\mathrm{S}}\right)$ (section 3.5.3) [12, 48, 49, 80, 98].

Once the avalanche is triggered, the temperature of the defect region $T(t)$ starts to increase because of the energy dissipated during the avalanche process. This rise in temperature is modeled by a thermal model incorporating a thermal resistance and thermal capacitance, $R_{\mathrm{TH}}$ and $C_{\mathrm{TH}}$ respectively [99], as shown in Fig. 3.10(c). 


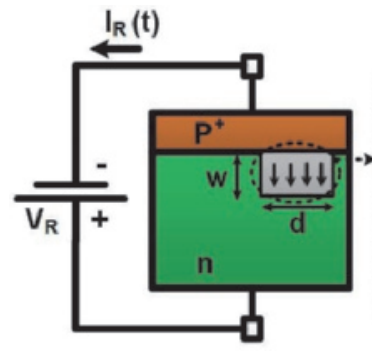

a)

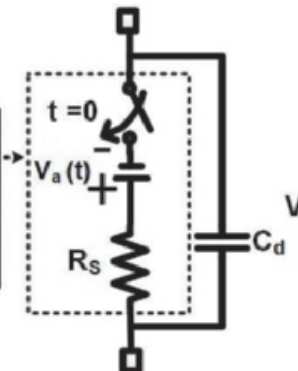

b)

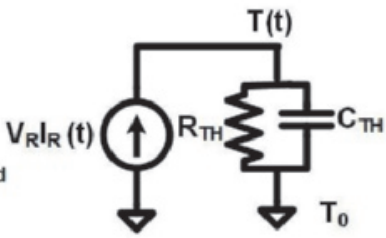

c)

Figure 3.10: (a) Schematic cross-section of a cylindrical defect of height $w$ and diameter $d$ inside a $\mathrm{p}^{+}-\mathrm{n}$ junction. (b) Circuit model for the defect comprising of avalanche sustaining voltage $V_{\mathrm{a}}$, series resistance $R_{\mathrm{S}}$, bistable switch and diode capacitance $C_{\mathrm{d}}$. (c) A thermal model to estimate the temperature of the defect region.

With Fig. 3.10(b), the RTS current $I_{R}(t)$ is:

$$
I_{\mathrm{R}}(t)=\frac{V_{\mathrm{R}}-V_{\mathrm{a}}(t)}{R_{\mathrm{S}}} .
$$

An elevated $T(t)$ results in an increased $V_{\mathrm{a}}(t)$ [49], thereby providing a negative feedback for the avalanche process. In these devices, the doping $N_{\mathrm{D}} \sim 10^{17}\left[\mathrm{~cm}^{-3}\right]$, and for such $N_{\mathrm{D}}$, the $V_{\mathrm{a}}$ can be approximated with a linear dependence on $T(t)[100,101]$ :

$$
V_{\mathrm{a}}(t)=V_{\mathrm{a} 0}+\beta \cdot\left(T(t)-T_{0}\right),
$$

where $\beta(\sim 5 \mathrm{mV} / \mathrm{K})$ is the thermal coefficient for the breakdown voltage [101], $T_{0}$ is the initial temperature at $t=0$ and $V_{\mathrm{a} 0}$ is $V_{\mathrm{a}}$ at $T=T_{0}$. The $T(t)$ due to self heating is:

$$
\begin{gathered}
T(t)=T_{\mathrm{f}}-\left(T_{\mathrm{f}}-T_{0}\right) \cdot e^{-t / \tau}, \\
T_{\mathrm{f}} \approx T_{0}+\frac{R_{\mathrm{TH}} V_{\mathrm{R}}\left(V_{\mathrm{R}}+\beta T_{0}-V_{\mathrm{a} 0}\right)}{R_{\mathrm{S}}+\beta R_{\mathrm{TH}} V_{\mathrm{R}}}, \\
\tau=\frac{R_{\mathrm{TH}} R_{\mathrm{S}} C_{\mathrm{TH}}}{R_{\mathrm{S}}+\beta R_{\mathrm{TH}} V_{\mathrm{R}}} .
\end{gathered}
$$

The physical variables in the above equations are:

- $T_{\mathrm{f}}$ is the temperature in thermal equilibrium at $t \rightarrow \infty$ assuming that the avalanche is not quenched.

- $R_{\mathrm{TH}}([\mathrm{K} / \mathrm{W}])$ is the thermal resistance of the avalanche region.

- $C_{\mathrm{TH}}([\mathrm{Ws} / \mathrm{K}])$ is the thermal capacitance of the avalanche region. 
An expression for $R_{\mathrm{TH}}=\left(T_{\mathrm{f}}-T_{0}\right) /\left(V_{\mathrm{R}} I_{\mathrm{R}}\right)$ has been derived in [49]:

$$
R_{\mathrm{TH}}=\frac{1}{\pi k d\left(\frac{w}{d}\right)}\left(\frac{w}{4 d} \sqrt{1+\left(\frac{w}{d}\right)^{2}}+\frac{1}{8} \ln \left(\frac{\sqrt{1+\left(\frac{w}{d}\right)^{2}}+\frac{w}{d}}{\sqrt{1+\left(\frac{w}{d}\right)^{2}}-\frac{w}{d}}\right)\right) .
$$

where $k(\sim 2 \mathrm{~W} / \mathrm{cm}-\mathrm{K})$ is the thermal conductivity of $\mathrm{Si}$ [12]. In these devices, for typical values of $w$ and $d$ (section 3.5.3), $\beta R_{\mathrm{TH}} V_{\mathrm{R}}$ is much smaller than $R_{\mathrm{S}}$, therefore $\tau \approx R_{\mathrm{TH}} C_{\mathrm{TH}}$ (Eq. (3.7c)).

Using Eqs. (3.5)-(3.7), $I_{R}(t)$ can be written as:

$$
I_{\mathrm{R}}(\mathrm{t})=\frac{V_{\mathrm{R}}-V_{\mathrm{a} 0}}{R_{\mathrm{S}}}-\frac{\beta \cdot\left(T_{\mathrm{f}}-T_{0}\right)}{R_{\mathrm{S}}} \cdot\left(1-e^{-t / \tau}\right) .
$$

The peak current $\left(I_{\text {peak }}\right)$ of this $I_{R}(t)$ is given by:

$$
I_{\text {peak }}=I_{\mathrm{R}}(\mathrm{t}=0)=\frac{V_{\mathrm{R}}-V_{\mathrm{a} 0}}{R_{\mathrm{S}}}=\frac{V_{\mathrm{EX}}}{R_{\mathrm{S}}} .
$$

It is important to note that $I_{\text {peak }}$ depends only on the initial excess bias $\left(V_{\mathrm{EX}}=V_{\mathrm{R}}-V_{\mathrm{a} 0}\right)$ and $R_{\mathrm{S}}$. From the slope of the measured $I_{\text {peak }}-V_{\mathrm{EX}}$ characteristics, the $R_{\mathrm{S}}$ can be estimated:

$$
\frac{1}{R_{\mathrm{S}}}=\frac{\mathrm{d} I_{\text {peak }}}{\mathrm{d} V_{\mathrm{R}}}=\frac{\mathrm{d} I_{\text {peak }}}{\mathrm{d} V_{\mathrm{EX}}} .
$$

Further, from the estimated $R_{S}$, the effective $d$ of the defects are estimated in section 3.5.3.

Eq. (3.9) indicates that $I_{R}(t)$ decays with a time constant $\tau \approx R_{\mathrm{TH}} C_{\mathrm{TH}}$. The decay time constant of $I_{R}(t)$ has been estimated in section 3.3; the results are shown here for completeness of the model.

In this section, two lumped defects in one diode are modeled: one causing the "decaying" amplitude RTSs and the other causing the "constant" amplitude RTSs. Multiple defects with similar breakdown voltage and resistance cannot be distinguished using TDA.

\subsubsection{Estimating $V_{\mathrm{a} 0}$}

As mentioned before, $V_{\mathrm{a} 0}$ is defined as the voltage at which $M=1$ at $T=T_{0}$ [49]. However, estimating this voltage is by no means straight forward. In chapter 2, an alternative definition of the experimental breakdown voltage $\left(V_{\mathrm{BR}}\right)$ was proposed; $V_{\mathrm{BR}}$ being the voltage at which $M=2$. This is estimated from the SMU measured $I-V$ characteristics and the RTS weighted $\mathrm{E}(D) \cdot A-V$ characteristics. The term $\mathrm{E}(D) \cdot A$ represents the average current of the RTS current pulses. The current during the on-state of the RTSs is due to impact ionization.

Both the SMU measured $I-V$ and the RTS weighted $\mathrm{E}(D) \cdot A-V$ curves were extrapolated (dashed lines in Fig. 3.11(a)). The intersection of these extrapolated curves is the voltage at which the RTS weighted current 
a)

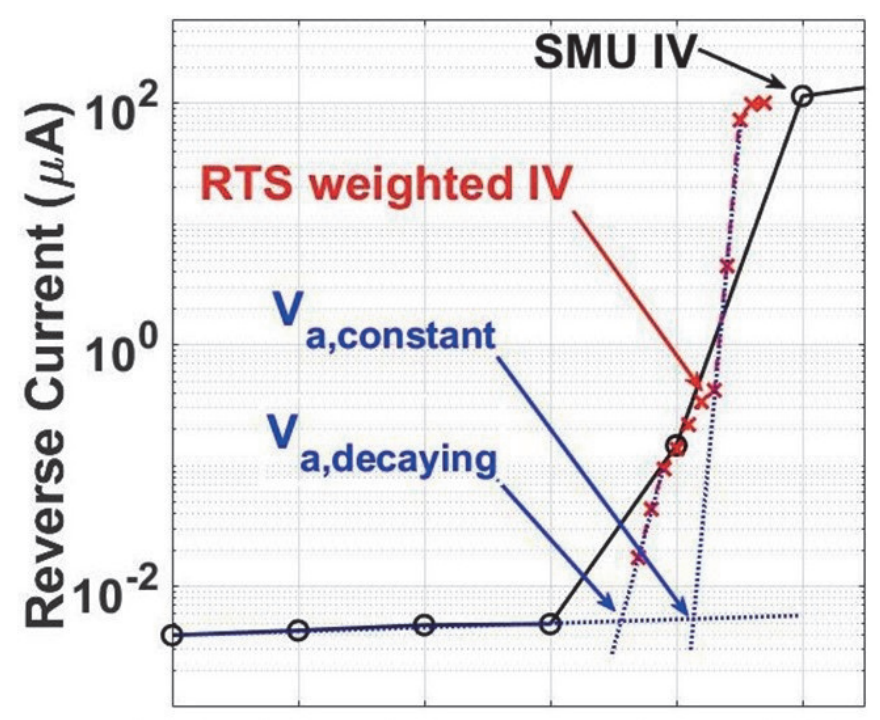

\section{$\begin{array}{llllll}13.4 & 13.5 & 13.6 & 13.7 & 13.8 & 13.9\end{array}$ \\ Reverse Voltage (V)}

\begin{tabular}{|c|c|c|}
\hline Diode & $\boldsymbol{V}_{\mathrm{a}, \text { decaying }}(\mathrm{V})$ & $\boldsymbol{V}_{\mathrm{a}, \text { constant }}(\mathrm{V})$ \\
\hline $\mathrm{J} 3$ & 13.81 & 13.87 \\
\hline $\mathrm{J} 6$ & 13.75 & 13.81 \\
\hline $\mathrm{J} 12$ & 13.73 & 13.80 \\
\hline $\mathrm{J} 20$ & 13.72 & 13.76 \\
\hline
\end{tabular}

Figure 3.11: Estimation of $V_{\mathrm{a} 0}$ for J6. (a) The intersection of SMU $I-V$ and RTS weighted $\mathrm{E}(D) \cdot A-V$ characteristics is the voltage at which $M=2$. From the intersection of two types of curves, the $V_{\mathrm{a} 0}$ for the defects causing "decaying" $\left(V_{\mathrm{a}, \text { decaying }}\right)$ and "constant" amplitude RTSs $\left(V_{\mathrm{a}, \text { constant }}\right)$ are estimated. (b) Summary of $V_{\mathrm{a} \text {,decaying }}$ and $V_{\mathrm{a} \text {,constant }}$ for all diodes.

contribution equals the leakage current component, hence $M=2$. This voltage is denoted as $V_{M=2}$ and will be used as approximation for $V_{\mathrm{a} 0}$. In reality, $V_{\mathrm{a} 0}$ would be somewhat lower than $V_{M=2}$.

There are two different types of defects in these diodes causing two types of RTSs and the $V_{\mathrm{a} 0}$ is different for both defects. Fig. 3.11(a) shows the example procedure for estimating $V_{\mathrm{a} 0}$ for both types of defects in $\mathrm{J} 6$, i.e. $V_{\mathrm{a} \text {,decaying }}$ and $V_{\mathrm{a} \text {,constant }}$; similar procedure was carried out for other diodes and the results are summarized in Fig. 3.11(b). The "constant" amplitude RTSs have a higher $V_{\mathrm{a} \text {,constant. These voltages are used in estimating }}$ $V_{\text {EX }}$ to calculate $R_{S}$. 

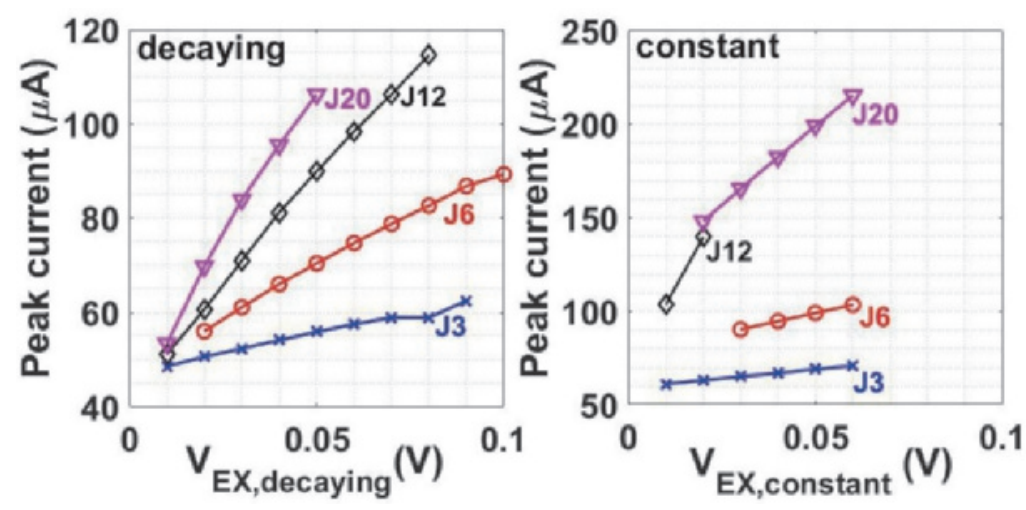

Figure 3.12: Mean peak current $\left(I_{\text {peak }}\right)$ of various diodes as a function of $V_{\mathrm{EX}}$ : (a) for the "decaying" amplitude RTSs. (b) for the "constant" amplitude RTSs. The y-axis scales are different for clarity.

\subsubsection{Peak current}

Fig. 3.12 shows the mean value of measured $I_{\text {peak }}$ (Fig. 3.5) as a function of $V_{\mathrm{EX}}\left(=V_{\mathrm{R}}-V_{\mathrm{a} 0}\right)$ of all diodes and for both types of RTSs. The mean values were estimated using all observed RTSs of each type at all bias conditions. It is observed that $I_{\text {peak }}$ increases with $V_{\mathrm{EX}}$.

The offset in $I_{\text {peak }}$ around $V_{\mathrm{EX}}=0$ is most likely because the actual $V_{\mathrm{a} 0}$ is lower than $V_{M=2}$. In the measurement duration of $1 \mathrm{~s}$ (section 3.2), no RTS phenomenon was observed at $V_{\mathrm{R}}<V_{\mathrm{a} 0}$.

The RTSs are caused by localized defects inside the devices [45, 49, 74]. The observed increase in $I_{\text {peak }}$ for larger diodes is most likely due to the spreading of avalanche to secondary defects, once a single defect is triggered. As the size of the diode increases, the number of defects causing RTS phenomena increases (see also appendix B). This could cause the avalanche triggered at a defect site to trigger secondary defects by two mechanisms [102]: 1) drift and diffusion of free carriers in lateral direction and 2) emission of secondary photons. As reported in [102], this spreading of avalanche occurs in a few tens of picoseconds. Fig. 3.13(a) illustrates this process.

Hence, the measured $I_{\text {peak }}$ could originate from breakdown at multiple defects (Fig. 3.13(b)):

$$
I_{\text {peak }}=\sum_{\mathrm{i}=1}^{\mathrm{n}} \frac{V_{\mathrm{R}}-V_{\mathrm{a}, \mathrm{i}}}{R_{\mathrm{S}, \mathrm{i}}}
$$

where $V_{\mathrm{a}, \mathrm{i}}$ and $R_{\mathrm{S}, \mathrm{i}}$ are the breakdown voltage and the series resistance of the $i^{\text {th }}$ defect.

\subsubsection{Series resistance and geometrical details of the defects}

As stated before, the resistance due to the partial neutralization of space charge in the avalanche region is referred to as the space-charge resistance 


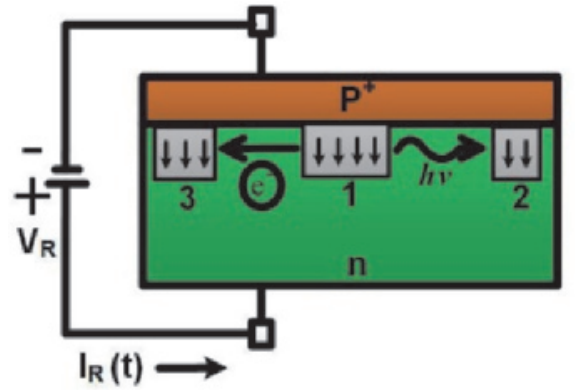

a)

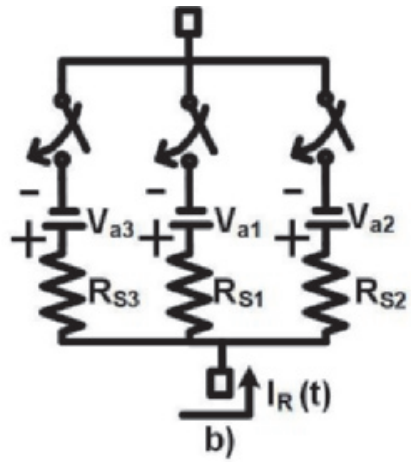

b)

Figure 3.13: a) Illustration of spreading of avalanche where multiple defects in a single diode are involved. First defect 1 is triggered, then defect 2 is triggered by secondary photons emitted by defect 1 and defect 3 by lateral transport of free carriers. b) Equivalent circuit model for modeling the spreading effect for three defects.

$\left(R_{\mathrm{S}}\right)$. Using Eq. (3.11) and Fig. 3.12, $R_{\mathrm{S}}$ was estimated for the two types of defects for all diodes. An example of the procedure for estimating $R_{\mathrm{S}}$ is shown in Fig. 3.14(a). The estimated $R_{\mathrm{S}}$ of defects in all diodes are shown in Fig. 3.14(b). The results indicate that the defects causing "decaying" amplitude RTSs have a higher $R_{\mathrm{S}}$ than the defects causing "constant" amplitude RTSs. The relatively low $R_{\mathrm{S}}$ for larger diodes can also be explained by a simultaneous breakdown of multiple defects (Fig. 3.13):.

$$
\frac{1}{R_{\mathrm{S}}}=\sum_{\mathrm{i}=1}^{\mathrm{n}} \frac{1}{R_{\mathrm{S}, \mathrm{i}}}
$$

Alternatively, each component in $R_{\mathrm{S}}$ can be described by the dimensions of a single defect and the properties of the diode (impurity concentration). For a defect inside a single sided abrupt $\mathrm{p}^{+}-\mathrm{n}$ junction, its $R_{\mathrm{S}, \mathrm{i}}$ is given by [12]:

$$
R_{\mathrm{S}, \mathrm{i}}=\frac{2\left(w-x_{\mathrm{A}}\right)^{2}}{\pi \epsilon_{\mathrm{S}} v_{\mathrm{S}} d_{\mathrm{i}}^{2}}
$$

where $w$ is the depletion region width, $x_{\mathrm{A}}$ is the avalanche region width where most of the multiplication takes place [12], $\epsilon_{\mathrm{s}}=1.04 \times 10^{-12} \mathrm{~F} / \mathrm{cm}^{2}$ is the permittivity of $\mathrm{Si}, v_{\mathrm{s}}=1 \times 10^{7} \mathrm{~cm} / \mathrm{s}$ is the saturation velocity of electrons and holes and $d_{\mathrm{i}}$ is the diameter of the $\mathrm{i}^{\text {th }}$ defect. For simplicity, $v_{\mathrm{S}}$ is assumed the same for electrons and holes $[12,74]$. For single-sided abrupt junctions: $x_{\mathrm{A}} \sim 0.3 w$ [74].

From the estimated $R_{S}$, the value of the effective diameter $d$ is estimated and results are tabulated in Fig. 3.14(c) for both types of defects for all devices. According to Eqs. (3.13) and (3.14), $d$ is an effective value possibly 

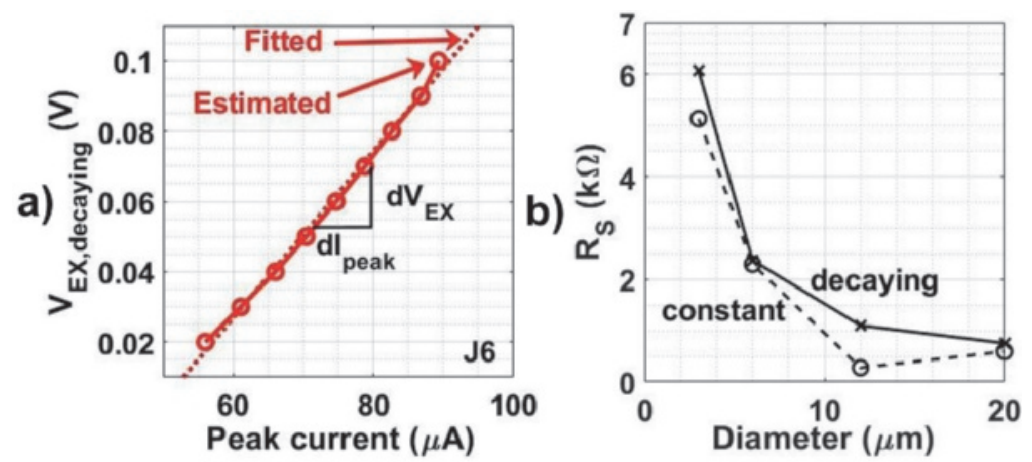

\begin{tabular}{|c|c|c|c|c|}
\hline Device & J3 & J6 & $\mathrm{J} 12$ & J20 \\
\hline decaying $d(\mu \mathrm{m})$ & 0.97 & 1.56 & 2.29 & 2.76 \\
\hline constant $d(\mu \mathrm{m})$ & 1.06 & 1.59 & 4.57 & 3.11 \\
\hline
\end{tabular}

Figure 3.14: a) Example procedure for estimating $R_{\mathrm{S}}=\mathrm{d} V_{\mathrm{EX}} / \mathrm{d} I_{\text {peak }}$ for "decaying" amplitude RTSs in J6. b) Estimated $R_{S}$ of the defects causing "decaying" and "constant" amplitude RTSs. c) Estimated diameter $d$ for both types of defects in all diodes. The $d$ for defect causing "constant" amplitude RTSs in J12 is larger than that in J20. This is also observed from optical measurements (appendix C).

obtained from the breakdown of many defects:

$$
d=\sqrt{\sum_{i=1}^{n} d_{i}^{2}}
$$

where $d_{\mathrm{i}}$ is the diameter of the $\mathrm{i}^{\text {th }}$ defect. The first observation from Fig. 3.14(c) is that the defects causing "decaying" amplitude RTSs have a relatively smaller $d$ than the defects causing "constant" amplitude ones. The second observation is the increasing effective size of the defects with the size of the device as discussed and explained by the spreading of avalanche to multiple defects in large diodes. The estimated $d$ is in the same order of magnitude as the reported values in the literature obtained from optical measurements [49, 52,74]. Also, the optical measurements on these devices confirmed this (appendix C).

\subsubsection{Thermal time constant}

Fig. 3.15 shows the estimated thermal time constant $\left(\tau \approx R_{\mathrm{TH}} C_{\mathrm{TH}}\right)$ of the "decaying" amplitude RTSs (section 3.3). The figure shows that $\tau$ increases with increasing $V_{E X, \text { decaying. }}$.

It was observed using the light emission properties of the defects that the $d$ remains almost constant over the instability region [49]. The $w$ increases with increasing $V_{\mathrm{EX} \text {,decaying }}$ and therefore the $R_{\mathrm{TH}}$ should increase with increasing $w / d$ (Eq. 3.8), also predicted in [49]. Also, $C_{\mathrm{TH}}$ increases 


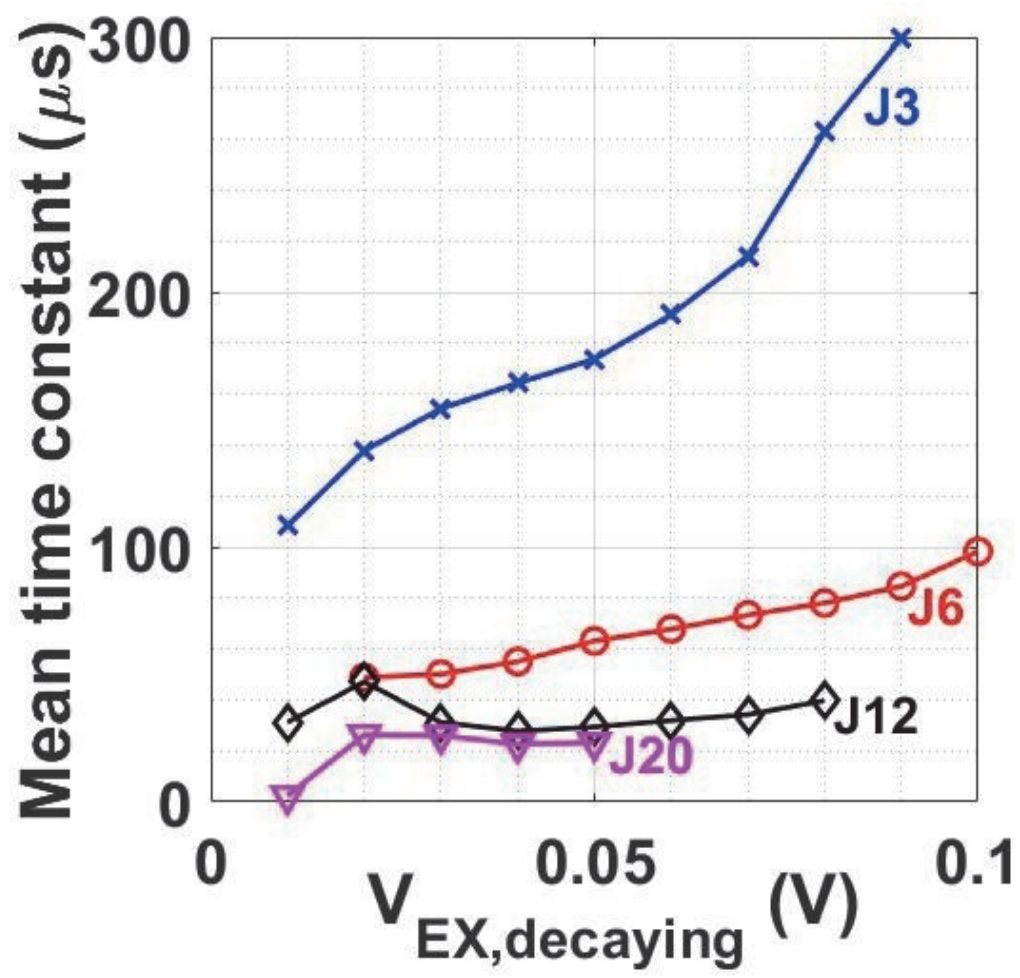

Figure 3.15: Mean thermal time constant $(\tau)$ of "decaying" amplitude RTSs as a function of $V_{\mathrm{EX} \text {,decaying. }}$.

proportionally with the volume of the defect [103]. This complies with

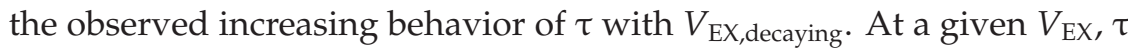
decreases for larger diodes because of larger $d$ (section 3.5.3).

\subsection{Conclusions}

An extensive time domain analysis of RTS phenomena in the diode current in avalanche was presented. Using the analysis results, the dependency of RTS parameters (amplitude and mean on-time fraction) on the size of the diode was shown. The amplitude of the RTSs was shown to increase with the area of the diode which was explained by the spreading of avalanche to more defects in larger diodes. The steep DC measured $I-V$ dependency close to breakdown was shown to be due to the steep dependency of ontime fraction of RTSs on bias voltage. Two different types of RTSs were observed in these diodes: a "decaying" and a "constant" amplitude type and a comprehensive analysis of both RTSs was done. It was proposed that the "decaying" amplitude RTSs are caused by defects having relatively high thermal impedance. A model for the defects causing these RTS phenomena from the literature was adopted, and some of the defect parameters were estimated using the time domain analysis. The ohmic series resistances 
of these defects were estimated to be hundreds of ohms to a few $\mathrm{k} \Omega$. The diameter of the defects was estimated to be in the order of a few $\mu \mathrm{m}$. Further, it was argued that the "decaying" amplitude RTSs are caused by relatively small area defects and the "constant" amplitude RTSs by relatively large area defects. The demonstrated analysis procedure for estimating a defect's series resistance and its dimensions can be readily used in other devices as well. 



\title{
DATA TRANSMISSION CAPABILITIES OF SILICON AVALANCHE MODE LEDS
}

\begin{abstract}
The data transmission capabilities of silicon avalanche mode lightemitting diodes (AMLEDs) were investigated and the results are correlated to the multiplication noise and leakage current. The incoming data were modulated using pulse position modulation and the biterror-rate (BER) and jitter in the transmitted data were measured. The results indicate an intrinsically low speed in terms of BER and jitter. From various size AMLEDs, temperature variations and optical excitations, it is shown that the speed can be improved by using AMLEDs with a (1) relatively high multiplication noise, (2) relatively high leakage current and (3) higher charge-per-bit. Design recommendations for the high speed AMLEDs are discussed.
\end{abstract}




\subsection{Introduction}

The significant overlap of the emission spectrum of silicon (Si) avalanche mode light-emitting diodes (AMLEDs) with the responsivity of Si photodetectors (PDs) makes them attractive for monolithic integration of optocouplers in standard CMOS [16, 17, 104, 105]. In [13], we have demonstrated optocoupling between Si AMLEDs and PDs in standard CMOS and analyzed the effects of the link length on the optocoupling efficiency. For digital data communication, the output of an AMLED needs to be modulated in response to the incoming data. The maximum data rate in such a system is typically limited by how fast the LED can be turned on and off $[9,106]$.

The small signal modulation speed of AMLEDs has been reported in tens of $\mathrm{GHz}[63,107]$, which is determined by the transit time $\tau_{\mathrm{t}} \sim w_{\mathrm{d}} / v_{\text {sat }}$, where $w_{\mathrm{d}}$ is the depletion region width and $v_{\text {sat }} \sim 10^{7} \mathrm{~cm} / \mathrm{s}$ is the saturation velocity of electrons and holes; $\tau_{t}$ is typically a few tens of ps. However, the large signal switching speed depends on the availability of free carriers in the multiplication region and triggering of a new avalanche event. Without any experimental proof, it was claimed that this speed is also in tens of $\mathrm{GHz}$ [108]. We show that triggering of a new avalanche event can be a relatively slow process. If an avalanche would not be triggered during transfer of a data bit, it leads to a bit-error in the communication link. In addition, the delay variation in turning on of avalanche leads to jitter in the transmitted data. Obviously, a high bit-error-rate (BER) and jitter are undesirable. In this work, it is shown that:

- AMLEDs with more defects have more multiplication noise and achieve higher speed.

- AMLEDs with a relatively high leakage current have a high number of free carriers to trigger an avalanche, which improves speed.

- Higher speed can also be obtained by driving AMLEDs at a higher bias, which increases the avalanche triggering probability, at the cost of increased power consumption.

Based on these results, design recommendations for high speed AMLEDs are discussed. The presented results are important for the implementation of low power optical links using AMLEDs [13, 41, 104, 105].

\subsection{Experimental AMLEDs and noise}

For our experiments, the AMLEDs were fabricated in a pure boron (PureB) technology, a schematic cross-section of which is shown in Fig. 4.1. The anode was formed by chemical vapor deposition of PureB in windows to the n-Si surface through an oxide layer. The n-Si substrate had a nominal doping concentration of $10^{15} \mathrm{~cm}^{-3}$ and an n-enhancement layer was formed 


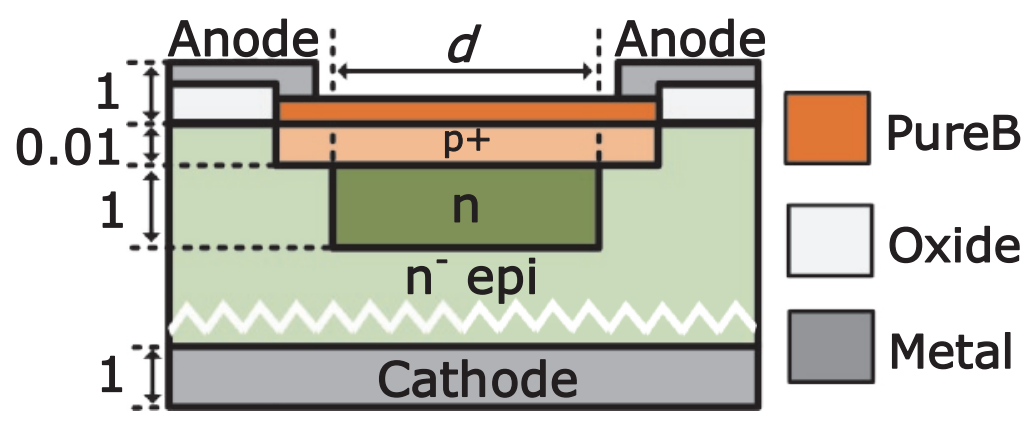

Figure 4.1: Schematic cross-section of a PureB AMLED (not to scale, all dimensions in $\mu \mathrm{m}$ ). The oxide is $\sim 0.3 \mu \mathrm{m}$ thick. Diameter (d) of an AMLED is defined by the dimensions of the n-enhancement layer.

under the anode by implanting phosphorus to achieve a local doping concentration of $10^{17} \mathrm{~cm}^{-3}$. A detailed description of the processing is given in [94] where similar PureB diodes were fabricated with the addition of a buried $\mathrm{n}^{+}$layer. Four circular diodes, on the same die were selected with diameters $(d)$ of $8 \mu \mathrm{m}, 15 \mu \mathrm{m}, 20 \mu \mathrm{m}$ and $30 \mu \mathrm{m}$; we label these diodes as $\mathrm{J} 8, \mathrm{~J} 15, \mathrm{~J} 20$ and $\mathrm{J} 30$ respectively.

Fig. 4.2 shows the DC I-V characteristics of the AMLEDs measured using a Keysight B2901A Source/Measure Unit (SMU) and 1 second integration time. The breakdown voltage $\left(V_{\mathrm{BR}}\right)$ of the AMLEDs, defined as the reverse voltage $\left(V_{R}\right)$ at which the reverse current $\left(I_{R}\right)$ starts to sharply increase, is $\sim 13.7 \mathrm{~V}$. An example of the light-emission from such an AMLED is also shown in the inset.

We have shown that in avalanche diodes, $I_{\mathrm{R}}$ exhibits Random Telegraph Signal (RTS) phenomena in the steep part of the $I-V$ characteristics (chapter 2) [68]. Initially close to $V_{\mathrm{BR}}$, the RTSs are short in duration. As the $V_{\mathrm{R}}$ increases, the RTSs become longer and their amplitude increases [69]. Ultimately, the RTSs disappear and the diode is in a continuously onstate. In the on-state, there is also multiplication noise $\left(\sigma_{M}\right)$ present in the avalanche current $[69,85]$.

The RTS phenomena were measured as done previously (Fig. 4.3) (chapter 3) [69]. We define $\sigma_{M}$ as the difference between the noise in the on-state of the device $\left(\sigma_{1}\right)$ and the measurement setup noise $\left(\sigma_{0}\right): \sigma_{\mathrm{M}}=$ $\sqrt{\sigma_{1}{ }^{2}-\sigma_{0}^{2}}$ (Fig. 4.3c) [85].

Fig. 4.4 shows an example of the measured transient $I_{R}$ for all AMLEDs in a continuous on-state. Fig. 4.5 shows the estimated $\sigma_{M}$ for all AMLEDs as a function of their diameter $(d)$. A higher $\sigma_{M}$ can be observed for larger AMLEDs which can be explained by the higher amount of defects in a larger device [69]; the noise generated by each defect is added up [97]. The linear dependency of $\sigma_{\mathrm{M}}$ on $d$ indicates that the number of defects increases linearly with the area of the diode. 


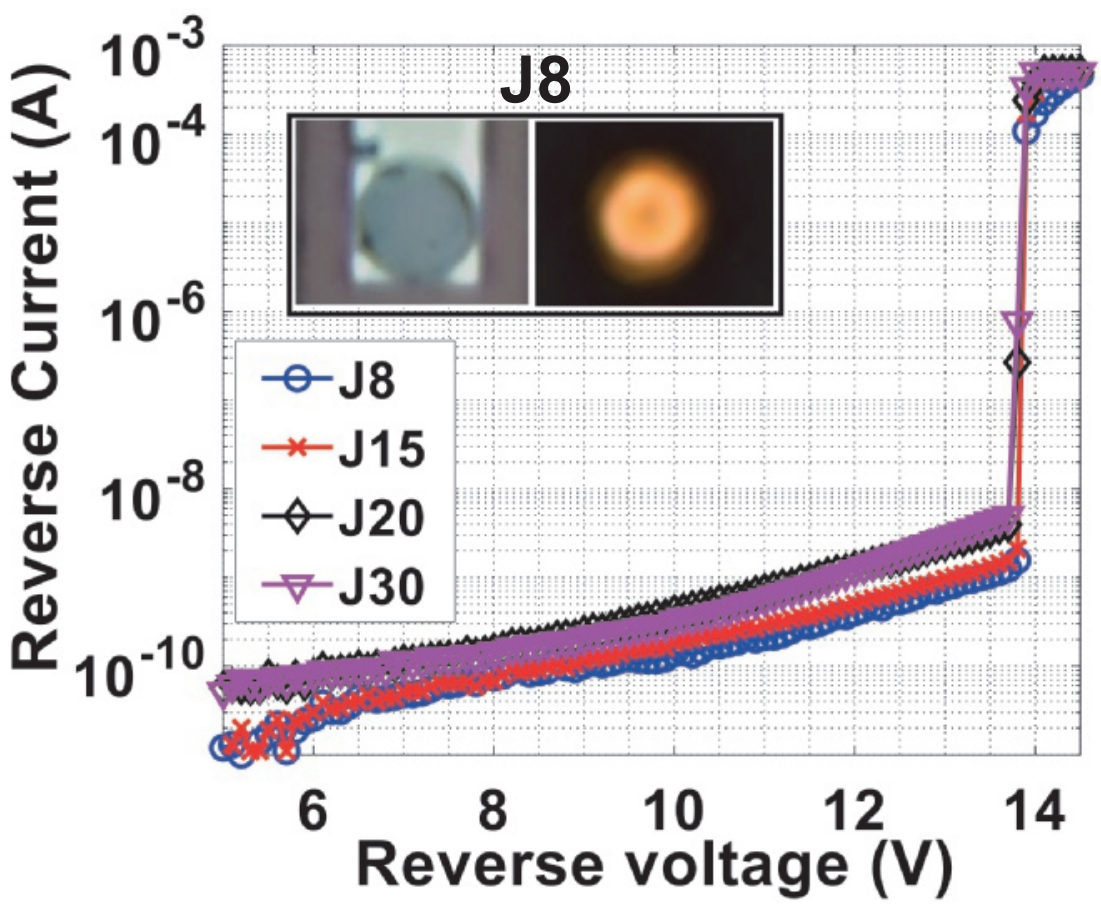

Figure 4.2: Measured reverse $I-V$ characteristics of PureB AMLEDs. Inset shows light emission from J8, biased at a reverse current of $1 \mathrm{~mA}$, captured using a Nikon D3100 camera with an integration time of 30 seconds.

a)

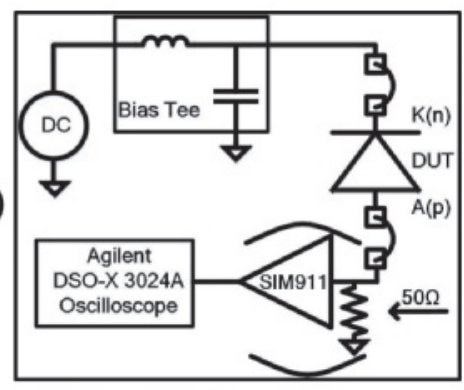

b)

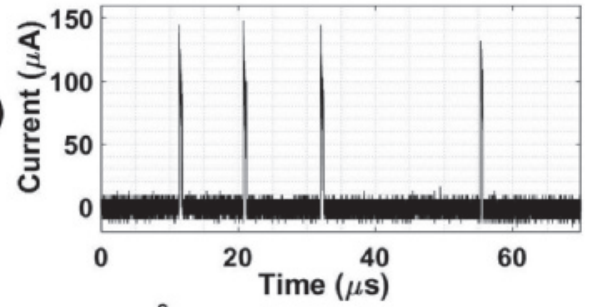

C)

Figure 4.3: (a) Random Telegraph Signal (RTS) measurement setup. (b) Measured RTSs in $I_{R}$. (c) Histogram of the measured RTSs. $\sigma_{0}$ is the noise from the experimental setup whereas $\sigma_{1}$ also includes multiplication noise $\left(\sigma_{M}\right)$. 

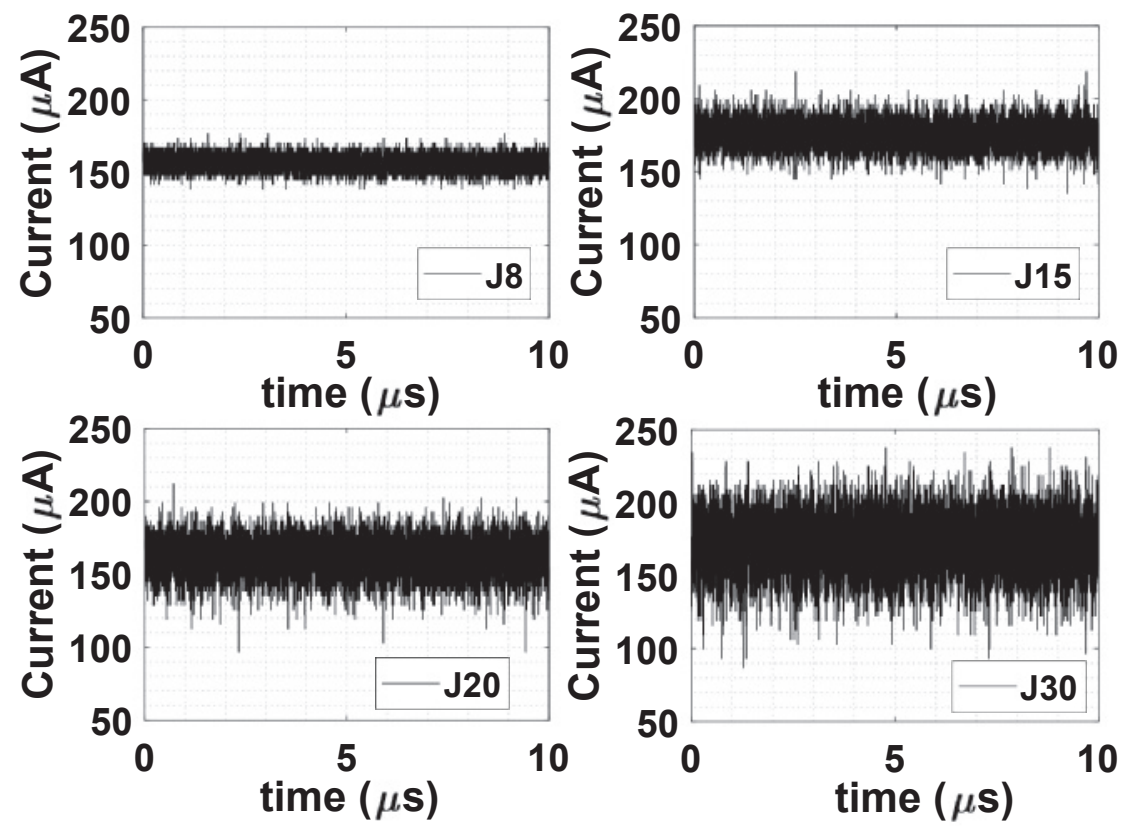

Figure 4.4: Example of measured transient avalanche currents when the AMLEDs are in on-state; these data include the measurement setup noise $\left(\sigma_{0}\right)$.

\subsection{Pulse position modulation speed}

\subsubsection{AMLED driver circuit}

Light is emitted from an AMLED only during avalanche [14]. For low power receivers in digital optical links using e.g. single-photon avalanche diodes, the LED should be switched between completely on and off conditions. Therefore, the voltage across an AMLED ( $V_{\text {AMLED }}$ ) should be modulated between below and above $V_{\mathrm{BR}}$ by driver circuit.

Fig. 4.6 shows the schematics of the driver circuit along with illustrative transient waveforms that we used to modulate $V_{\text {AMLED }}$. The circuit can also limit the charge-per-bit $\left(Q_{b}\right)$ through the AMLED. Limiting $Q_{b}$ limits the energy-per-bit $\left(E_{\mathrm{b}}\right)$, which is an important figure-of-merit in optical links.

We briefly describe the functionality of this circuit. For further details of this circuit, we refer the reader to chapter 5 [71], in which we have demonstrated an integrated implementation of this driver circuit. Initially the AMLED is biased below $V_{\mathrm{BR}}$ and then $V_{\mathrm{AMLED}}=V_{\mathrm{LOW}}=V_{\mathrm{BIAS}}-V_{\mathrm{DD}}$. First the reset switch M1 is opened using the control signal RST. Then the control signal IN is set high and $V_{\text {AMLED }}$ initially increases to:

$$
V_{\mathrm{AMLED}}=V_{\mathrm{BIAS}}-V_{\mathrm{DD}} \cdot\left(\frac{C_{\mathrm{P}}}{C_{\mathrm{P}}+C_{\mathrm{Q}}}\right)
$$




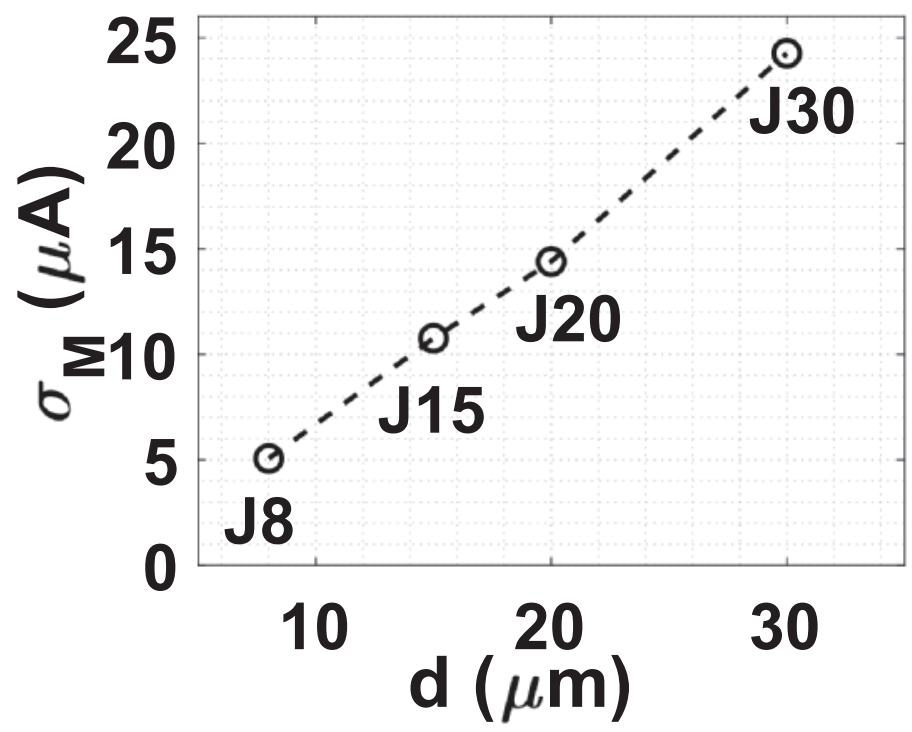

Figure 4.5: Multiplication noise $\left(\sigma_{M}\right)$ as a function of diameter $(d)$ of AMLEDs.

where the total parasitic capacitance $C_{P}=C_{\text {PAR }}+C_{\text {AMLED }}$, with $C_{\text {PAR }}$ the circuit parasitic capacitance and $C_{\text {AMLED }}$ the AMLED capacitance. The initial excess bias $\left(V_{\mathrm{EX}, \mathrm{i}}=V_{\mathrm{AMLED}}-V_{\mathrm{BR}}\right)$ is:

$$
V_{\mathrm{EX}, \mathrm{i}}=V_{\mathrm{BIAS}}-V_{\mathrm{DD}} \cdot\left(\frac{C_{\mathrm{P}}}{C_{\mathrm{P}}+C_{\mathrm{Q}}}\right)-V_{\mathrm{BR}}
$$

The initial $I_{\mathrm{AMLED}} \approx V_{\mathrm{EX}, \mathrm{i}} / R_{\mathrm{AMLED}}$ where $R_{\mathrm{AMLED}}$ is the resistance of the AMLED, and $I_{\mathrm{AMLED}}$ charges $C_{\mathrm{Q}}$, thereby reducing $V_{\mathrm{AMLED}}$ and $I_{\mathrm{AMLED}}$. As $V_{\mathrm{AMLED}}$ reduces to $V_{\mathrm{BR}}$, the avalanche is quenched. The maximum $Q_{\mathrm{b}}$ equals $C_{\mathrm{Q}} V_{\mathrm{EX}, \mathrm{i}}$ :

$$
Q_{\mathrm{b}}=\int_{0}^{T_{\mathrm{ON}}} I_{\mathrm{AMLED}}(\mathrm{t}) \mathrm{dt} \leqslant \mathrm{C}_{\mathrm{Q}} V_{\mathrm{EX}, \mathrm{i}} \leqslant C_{\mathrm{Q}} V_{\mathrm{DD}}
$$

where $T_{\mathrm{ON}}$ is the pulse width of the control signal IN.

Due to the relatively low internal quantum efficiency of AMLEDs $\left(10^{-5}\right)$ [71], high speed optical measurements are not possible using conventional photodetectors. Here, we measure $I_{\text {AMLED }}$ as an indication of the light emission. The relation between $I_{\mathrm{AMLED}}$ and the light emission from AMLEDs is discussed in section 4.3.7.

In this work, the circuit of Fig. 4.6(a) was designed on a printed circuit board (PCB) which, in contrast to the CMOS integrated implementation of [71], resulted in $C_{P} \sim 20 \mathrm{pF}$. 


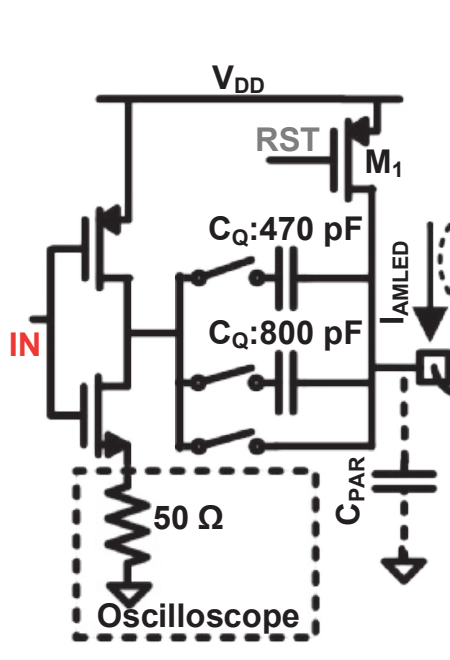

a)

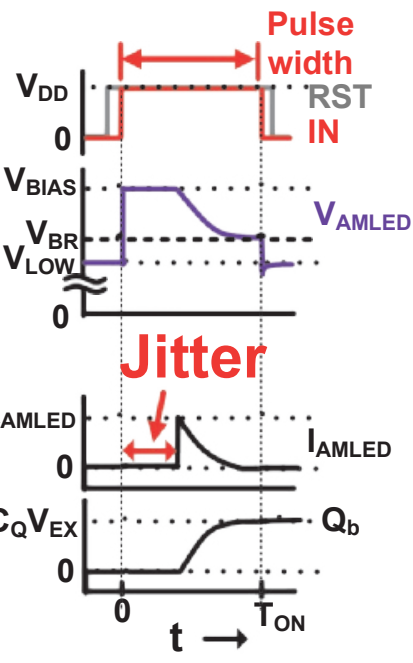

b)

Figure 4.6: (a) Schematic layout of an AMLED driver circuit to measure modulation speed [71]. The $I_{\text {AMLED }}$ was measured directly on the oscilloscope. In our experimental setup, three $C_{Q}$ settings were incorporated: 470 $\mathrm{pF}, 800 \mathrm{pF}$ and short $\left(C_{\mathrm{Q}} \rightarrow \infty\right)$. A simplified model for the AMLED is also shown [71]. (b) Illustrative transient waveforms.

\subsubsection{Pulse position modulation and bit error rate}

AMLEDs have a relatively low efficiency and therefore for fast and low power receivers, they should be integrated with highly sensitive photodetectors such as single-photon avalanche diodes (SPADs) [109]. SPADs are $\mathrm{p}-\mathrm{n}$ junctions biased above breakdown where an incoming photon can trigger an avalanche. The current then swiftly rises to macroscopic levels which can be detected using readout circuits [88].

For data communication applications using SPADs, pulse position modulation (PPM) is a modulation scheme of choice [109]. Fig. 4.7 illustrates the transient waveforms of the control signal IN of our driver circuit for a two level PPM. In the transmitter, if an avalanche is not triggered during a data bit, there is no light emission and consequently a bit error occurs in the transmitted data. Therefore, the BER is defined as the fraction of the number of data bits where no avalanche was detected, as illustrated in Fig. 4.7 (Note that BER $>0.5$ is possible according to this definition).

We varied $V_{\text {LOW }}$ by adjusting $V_{\text {BIAS }}$ and $V_{\text {DD }}$ in the driver circuit. As a result, the leakage current of the AMLED in the off-state is tuned. $V_{\text {BIAS }}$ and $V_{\mathrm{DD}}$ were also adjusted to achieve different $V_{\mathrm{EX}, \mathrm{i}}$ for each $V_{\mathrm{LOW}}$ (Eq. (4.2)). We will show in section 4.3.4 that as $V_{\mathrm{LOW}}$ or $V_{\mathrm{EX}, \mathrm{i}}$ increases, the BER decreases.

Furthermore, to study the effect of varying $Q_{b}$, the $C_{Q}$ was varied between three settings (Fig. 4.6(a)): $470 \mathrm{pF}, 800 \mathrm{pF}$ and $\mathrm{C}_{\mathrm{Q}} \rightarrow \infty$ (implemented as a short yielding no quenching). During an avalanche event, 
a)

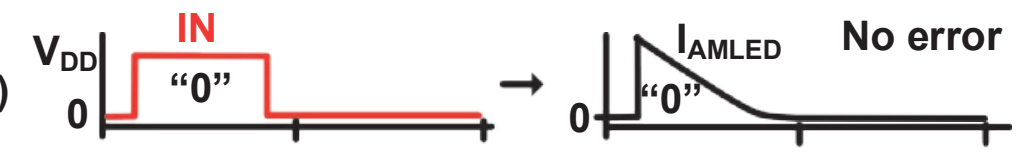

b)

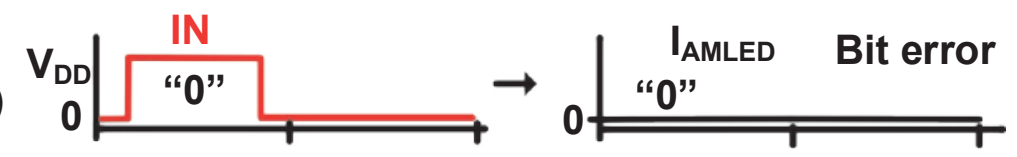

c)

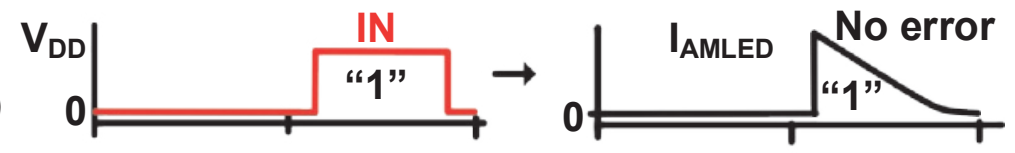

d)

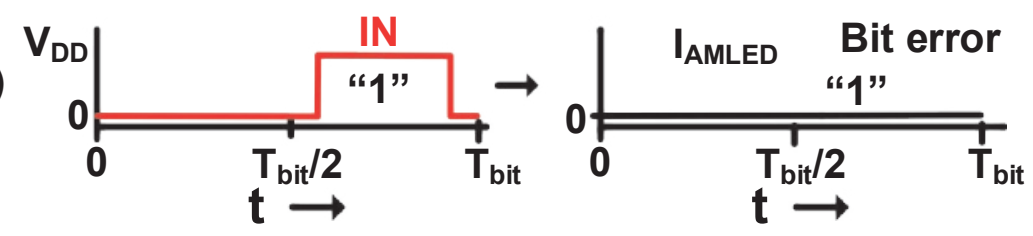

Figure 4.7: (Left) Illustrative transient waveforms of the control signal IN for bit " 0 " in $(a, b)$ and for bit " 1 " in $(c, d)$ when data are modulated using a two-level PPM; $T_{\text {bit }}=1 / f_{\mathrm{s}}$ is the bit duration time. (Right) Illustrative example of BER: For bit " 0 ", avalanche is triggered in (a) and therefore there is no bit error in the transmitted data whereas avalanche is not triggered in (b) causing a bit error. Similarly for bit " 1 ", there is no bit error in (c) whereas (d) causes a bit error. Hence, (b) and (d) contribute to BER.

some of the carriers flowing through the depletion region of the AMLED can get trapped. These trapped carriers are randomly released, causing free carriers that may trigger another avalanche event. This phenomenon is well-known as "afterpulsing" in SPADs [88], and is an undesired phenomenon in SPADs limiting their count rate [88]. However as shown in section 4.3.4, the mechanism yielding afterpulsing in SPADs helps to reduce the BER in AMLEDs.

To test the performance of the AMLEDs, a Pseudo Random Bit Sequence (PRBS) with a length of $2^{10}$ bits was generated. The control signals (IN and RST in Fig. 4.6(b)) for PRBS data were generated using a Keysight 33200A dual channel arbitrary waveform generator at a data rate $\left(f_{\mathrm{s}}\right)$ of $100 \mathrm{kbps}$.

Fig. 4.8 summarizes all biasing settings used in our measurements. The transient data at the oscilloscope were acquired at $2 \mathrm{GS} / \mathrm{s}(0.5 \mathrm{~ns}$ resolution). The performance of the AMLEDs was measured in terms of jitter and BER. The jitter is defined as the time delay between the time at which $V_{\text {AMLED }}>V_{\text {BR }}$ and the turn-on of avalanche, see Fig. 4.6(b). In our measurements, due to the used PRBS, the BER can be reliably measured down to $10^{-3}$ and therefore is lower limited to $10^{-3}$. 


\begin{tabular}{|c|c|c|c|}
\hline $\mathbf{V}_{\mathbf{D D}}(\mathbf{V})$ & $\mathbf{V}_{\text {BIAS }}(\mathbf{V})$ & $\mathbf{V}_{\text {LOW }}(\mathbf{V})$ & $\sim \mathbf{V}_{\text {EX,i }}(\mathbf{V})$ \\
\hline 6 & 17.6 & 11.6 & 3.9 \\
\hline 7 & 18.6 & 11.6 & 4.9 \\
\hline 8 & 19.6 & 11.6 & 5.9 \\
\hline 5 & 17.6 & 12.6 & 3.9 \\
\hline 6 & 18.6 & 12.6 & 4.9 \\
\hline 7 & 19.6 & 12.6 & 5.9 \\
\hline 4 & 17.6 & 13.6 & 3.9 \\
\hline 5 & 18.6 & 13.6 & 4.9 \\
\hline 6 & 19.6 & 13.6 & 5.9 \\
\hline
\end{tabular}

Figure 4.8: Bias settings of the driver circuit. For all three $C_{\mathrm{Q}}$ settings, by tuning $V_{\mathrm{DD}}$ and $V_{\mathrm{BIAS}}$, different values of $V_{\mathrm{LOW}}$ and $V_{\mathrm{EX}, \mathrm{i}}$ are obtained.

\subsubsection{Functionality}

Fig. 4.9 demonstrates an example of the measured $I_{\text {AMLED }}$ for J8 and J30; J8 shows a larger BER $(\sim 0.98)$ than J30 $(\sim 0.2)$ because of a higher multiplication noise in the latter (see section 4.3.4).

An ideal probability density function (PDF) of jitter for a two level PPM would be two dirac delta functions at the time instants of the rising edge of $V_{\text {AMLED }}$ (Fig. 4.6(b) and Fig. 4.7). However, in Fig. 4.9 large jitter in the turn-on time in both AMLEDs can be observed. This is because of a lack of free carriers and a defect count in these devices to trigger an avalanche. The PDFs of jitter are used in sections 4.3.5, 4.3.6 and 4.4.1 to demonstrate the effect of the leakage current and defects on the speed of AMLEDs.

\subsubsection{BER}

The probability of triggering of an avalanche during a pulse when $V_{\text {AMLED }}>$ $V_{\mathrm{BR}}$ depends on the number of free carriers in the depletion region $\left(N_{\mathrm{d}}\right)$ and the probability that a free carrier triggers avalanche $\left(P_{\mathrm{a}}\right)$. For a related analysis, a model of the triggering phenomenon was reported to explain the dark count rate of the SPADs [87]. In that model, the arrival of free carriers in the depletion region was modeled by a Poisson process. The probability that an avalanche is triggered $\left(P_{\mathrm{d}}\right)$ in a pulse of duration $T_{\mathrm{ON}}$ is given by [87]:

$$
\begin{gathered}
P_{\mathrm{d}}=1-\mathrm{e}^{-N_{\mathrm{d}} \cdot P_{\mathrm{a}}}, \\
\text { with } N_{\mathrm{d}}=N_{\mathrm{d} 1}+N_{\mathrm{d} 2}+N_{\mathrm{t} 1}+N_{\mathrm{t} 2}+N_{\text {opt }}
\end{gathered}
$$



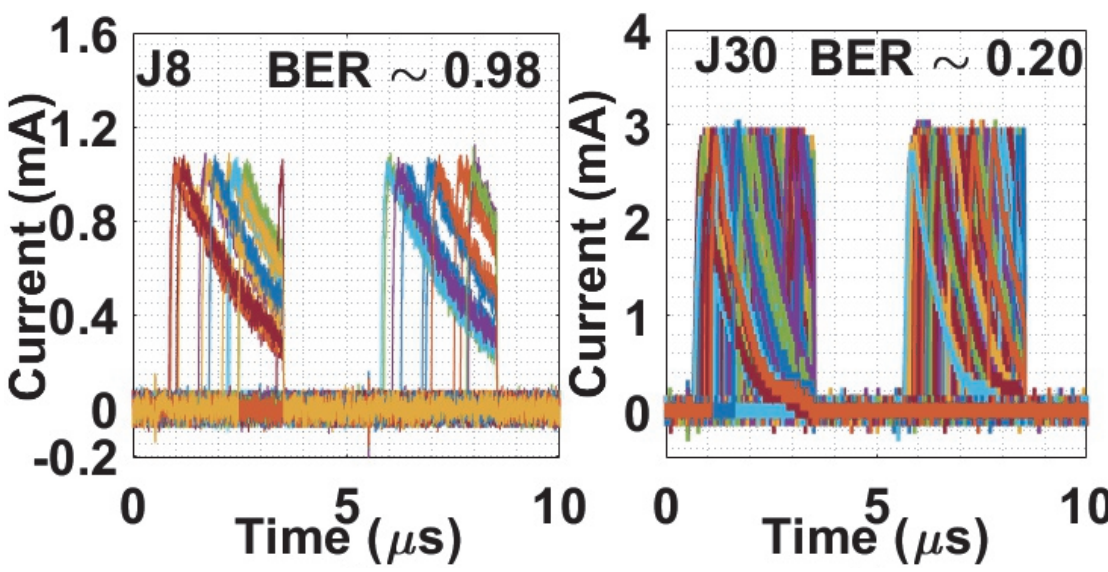

Figure 4.9: Measured transient waveforms of $I_{\text {AMLED }}$ at $C_{\mathrm{Q}}=800 \mathrm{pF}, V_{\text {BIAS }}$ $=17.6 \mathrm{~V}$ and $V_{\mathrm{DD}}=5 \mathrm{~V}$ for $\mathrm{J} 8$ and J30. The data have been superimposed for all $2^{10}$ bits. Y-axis scales are different for clarity.

$$
\text { and } \quad P_{\mathrm{a}}=1-\mathrm{e}^{-\frac{V_{\mathrm{EX}}}{\eta V_{\mathrm{BR}}}} \text {. }
$$

$$
\text { Further, } \quad B E R=1-P_{\mathrm{d}}=\mathrm{e}^{-N_{\mathrm{d}} \cdot P_{\mathrm{a}}} \text {. }
$$

In the above equations, $N_{\mathrm{d} 1}=I_{\mathrm{R}} T_{\mathrm{ON}} / q_{\mathrm{e}}$ is the number of carriers generated during the pulse where $I_{\mathrm{R}}$ is the leakage current and $q_{\mathrm{e}}$ is the elementary charge. Some of the carriers generated before the arrival of the pulse could remain in the depletion region due to the finite bandwidth of the AMLED; $N_{\mathrm{d} 2}$ is the number of those carriers. $N_{\mathrm{t} 1}$ is the number of released carriers from traps during the pulse (causing "afterpulsing" in SPADs). Similar to $N_{\mathrm{d} 2}, N_{\mathrm{t} 2}$ is the number of carriers that are released from traps before the arrival of the pulse. $N_{\text {opt }}$ is the number of optically generated carriers (section 4.3.6). Finally, $\eta$ is a fit parameter that depends on the defect density, temperature and device properties [87].

$N_{\mathrm{d}}$ and $P_{\mathrm{a}}$ are functions of the bias, temperature, device properties and driver circuit operating conditions. Exact solutions of these functions are difficult to obtain. However, the elegance of this model is its ability to explain the observed trends [87].

Fig. 4.10 shows the measured BER for all AMLEDs at different bias conditions (Fig. 4.8) in case of PPM. The BER

1. decreases with increasing $V_{\text {LOW }}$ at a given $V_{\text {BIAS, }}$

2. decreases with increasing $V_{\text {BIAS }}$ at a given $V_{\text {LOW, }}$

3. reduces for a higher $C_{Q}$, and

4. is lower for larger diodes. 

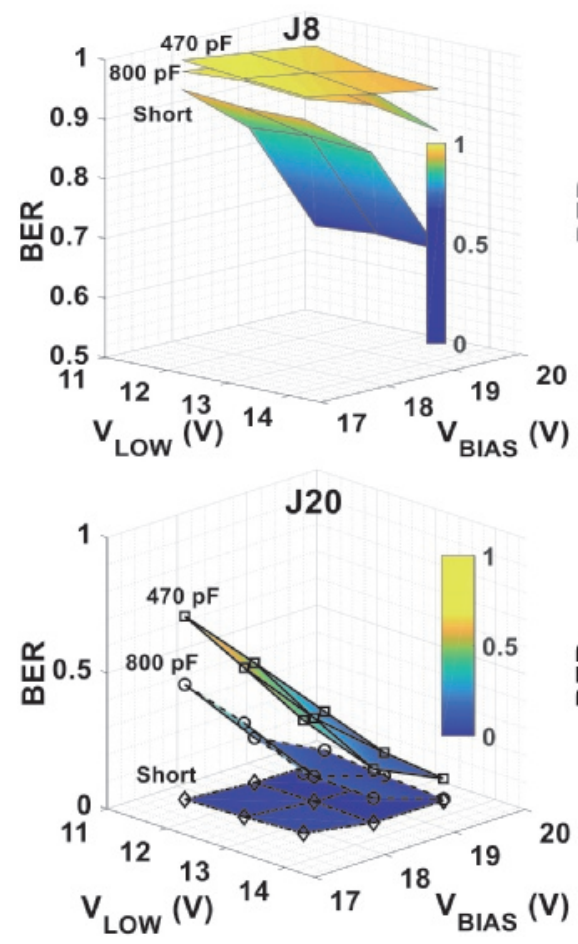

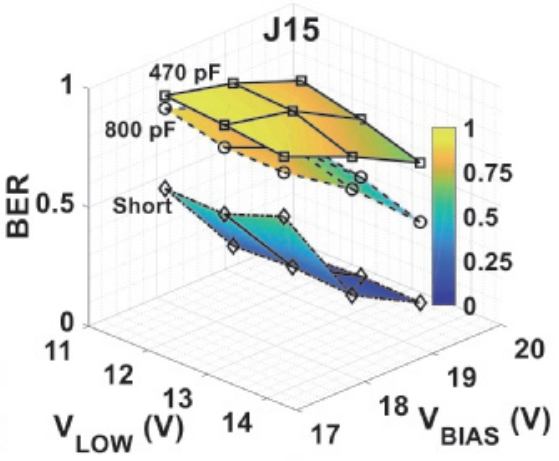

$\mathrm{J} 30$

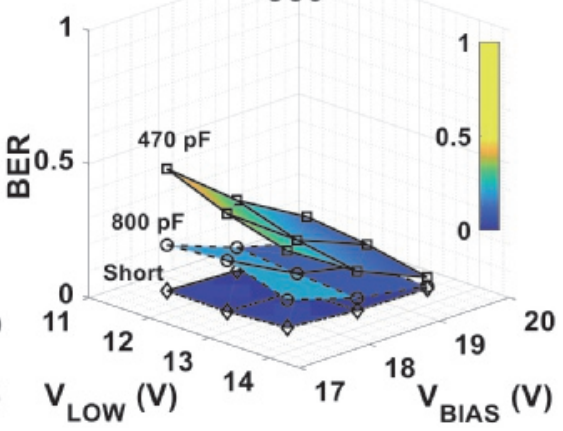

Figure 4.10: Measured BER for all AMLEDs as a function of $V_{\mathrm{LOW}}, V_{\mathrm{BIAS}}$ and $C_{\mathrm{Q}}$. BER is shown on a linear scale. For J8, the axis representing BER has a difference scale to enhance clarity.

The relatively high BER is due to a low defect density, therefore low $P_{\mathrm{a}}$, in these AMLEDs. The defect density is low because of the circular geometry and the presence of the implicit guard ring (Fig. 4.2) [94].

The decreasing BER for a higher $V_{\text {LOW }}$ (at a fixed $V_{\text {BIAS }}$ ) is because of an increase of $N_{\mathrm{d} 2}$. A higher $V_{\text {BIAS }}$ (at a fixed $V_{\text {LOW }}$ ) causes a higher $N_{\mathrm{d} 1}$ and a higher $P_{\mathrm{a}}$ (Eq. (4.4c)), consequently resulting in a lower BER.

A higher $C_{Q}$ causes a higher $Q_{\mathrm{b}}$ (Eq. (4.3)) and therefore more carriers are trapped during an avalanche event. These trapped carriers can be released during the next bit, increasing the probability of another avalanche. This effect results in a lower BER for a higher $Q_{b}$.

As the size of an AMLED increases, the multiplication noise increases (Fig. 4.5) indicating an increasing number of defects for larger diodes. This results in a higher $P_{\mathrm{a}}$ in Eq. 4.4(d) and therefore in a lower BER for larger devices.

When driving AMLEDs with $C_{\mathrm{Q}} \rightarrow \infty$, the BER further reduces. However, a higher amount of energy is then dissipated in the AMLEDs which increases its temperature. An elevated temperature results in a higher $V_{\mathrm{BR}}$; an example of this self-heating can be observed from the measured $I_{\text {AMLED }}(t)$ in Fig. 4.11. A higher $V_{\text {BR }}$ reduces $P_{\mathrm{a}}$ (Eq. 4.4(c)) which tends to increase the BER (Eq. 4.4(d)). Therefore, BER cannot be reduced by 


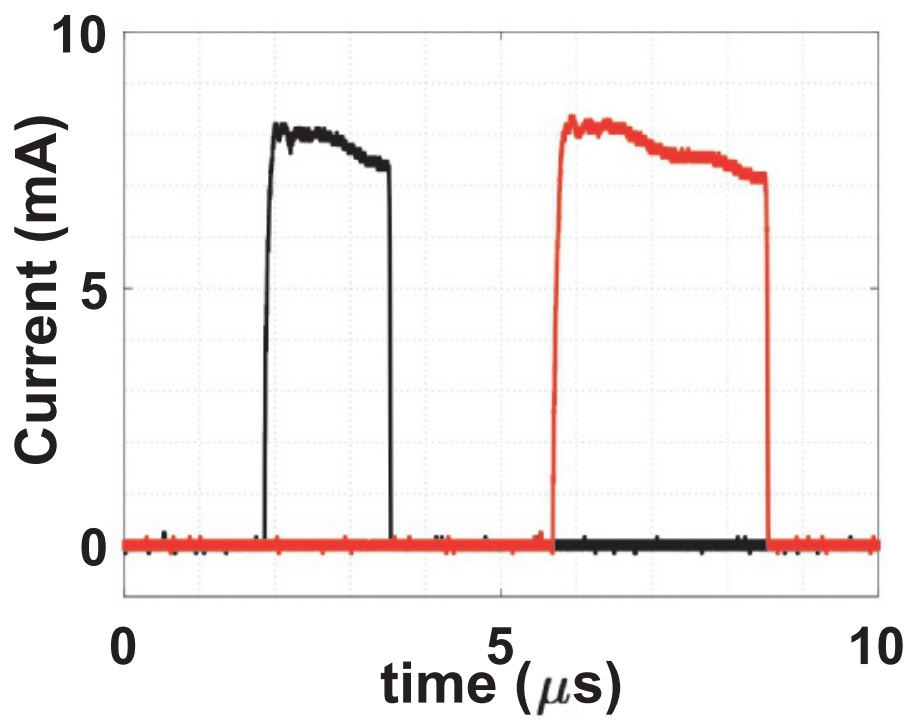

Figure 4.11: Example of self heating in J30: $V_{\mathrm{DD}}=8 \mathrm{~V}, V_{\mathrm{BIAS}}=19.6 \mathrm{~V}$ and the driver circuit was operated without any $C_{\mathrm{Q}}$ (short). $I_{\mathrm{AMLED}}$ reduces with time due to the self-heating of the AMLED.

operating AMLEDs at a very high $Q_{\mathrm{b}}$ because of this self-heating effect.

In conclusion, for a given AMLED, a reduced $Q_{b}$ causes a high BER which implies a constraint to reduce $Q_{\mathrm{b}}$ for an optical link ${ }^{1}$. Also, the $Q_{\mathrm{b}}$ cannot be arbitrarily increased because of increasing power consumption and self-heating.

\subsubsection{Effect of temperature}

The parameters $N_{\mathrm{d}}$ and $P_{\mathrm{a}}$ can be increased by operating the AMLEDs at elevated temperatures. The effect of temperature on the $I-V$ characteristics of a circular $12 \mu \mathrm{m}$ diode (J12) on the same die is shown in Fig. 4.12(a).

Using the same driver circuit and PRBS data, BER and jitter were measured at three temperatures for J12. By adjusting $V_{\mathrm{BIAS}}$ and $V_{\mathrm{DD}}, V_{\mathrm{LOW}}$ was varied between $V_{\mathrm{BR}}-0.1 \mathrm{~V}, V_{\mathrm{BR}}-1.1 \mathrm{~V}$ and $V_{\mathrm{BR}}-2.1 \mathrm{~V}$ at all temperatures (Fig. 4.8). Fig. 4.12(b) shows a lower BER at higher temperatures, which is due to a higher $N_{\mathrm{d}}$ and $P_{\mathrm{a}}$.

Fig. 4.13 shows an example of the jitter PDFs at three temperatures. Due to an increased $N_{\mathrm{d}}$ and $P_{\mathrm{a}}$, an avalanche is triggered faster at higher temperatures. Consequently, a lower standard deviation of the jitter PDFs can be observed at higher temperatures.

The quantum efficiency for high energy photons emitted from AMLEDs has a negative temperature coefficient [110], therefore a higher $Q_{b}$ would

\footnotetext{
${ }^{1}$ We focused only on the transmitter, in general $Q_{\mathrm{b}}$ is also lower limited because of the signal-to-noise ratio requirements at the receiver side.
} 


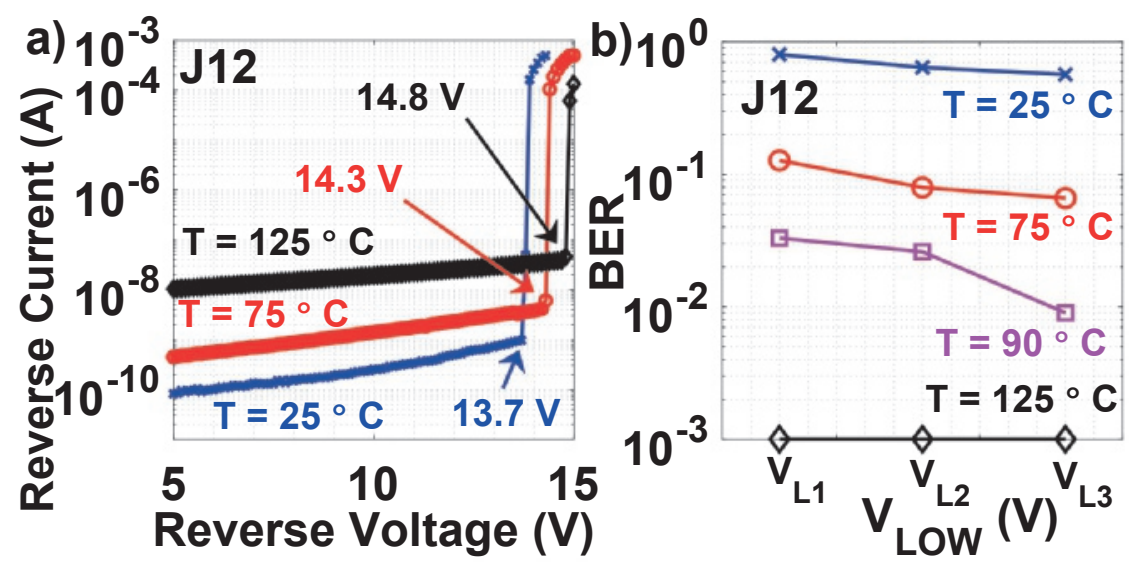

Figure 4.12: (a) $I-V$ characteristics of J12 for three different temperatures. Different $V_{\mathrm{BR}}$ at all temperatures are indicated. (b) Measured BER for diode $\mathrm{J} 12$ at $V_{\mathrm{BIAS}}=V_{\mathrm{BR}}+5.9 \mathrm{~V}, \mathrm{C}_{\mathrm{Q}}=470 \mathrm{pF} . \mathrm{V}_{\mathrm{L} 1}=V_{\mathrm{BR}}-2.1 \mathrm{~V}, \mathrm{~V}_{\mathrm{L} 2}=V_{\mathrm{BR}}-1.1$ $\mathrm{V}$ and $\mathrm{V}_{\mathrm{L} 3}=V_{\mathrm{BR}}-0.1 \mathrm{~V}$ are the $V_{\mathrm{LOW}}$ at each temperature. The BER is shown on a logarithmic scale for clarity.

be required at higher temperatures to ensure the same number of photons at the receiver of an optical link.

\subsubsection{Effect of external illumination}

To demonstrate the effect of increasing $N_{\mathrm{d}}$ without changing $P_{\mathrm{a}}$, free carriers were generated using optical illumination. An increasing $N_{\mathrm{d}}$ results in a lower BER (Eq. 4.4(d)).

An external LED with an emission spectrum around $650 \mathrm{~nm}$ was used to illuminate $\mathrm{J} 12$ at $\mathrm{T}=25^{\circ} \mathrm{C}$. Fig. 4.14 (a) shows the measured short circuit

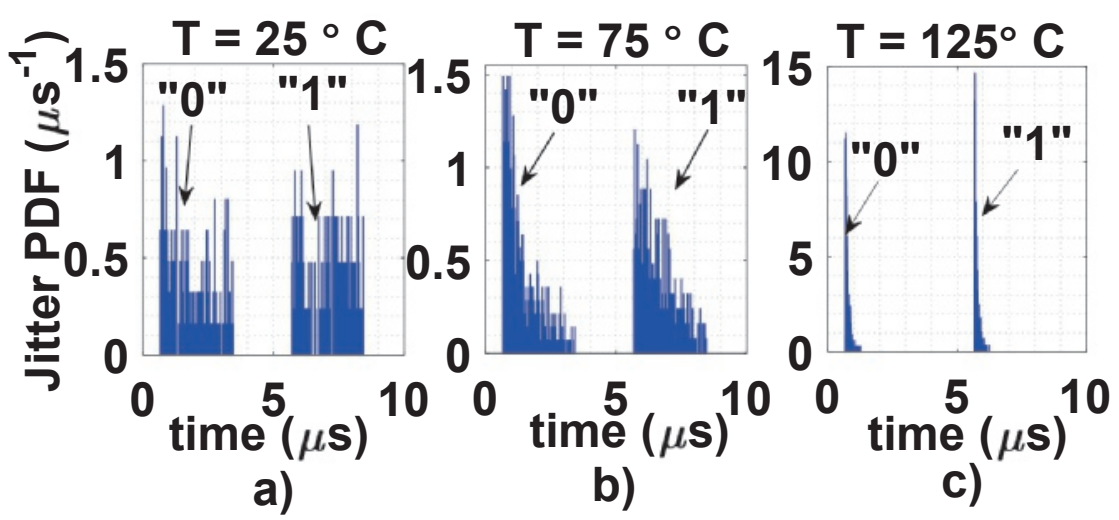

Figure 4.13: Measured PDFs of jitter for bit " 0 " and bit " 1 " for J12 at three temperatures. At all temperatures, $V_{\mathrm{BIAS}}=V_{\mathrm{BR}}+5.9 \mathrm{~V}, \mathrm{~V}_{\mathrm{L} 1}=V_{\mathrm{BR}}-2.1 \mathrm{~V}$ and $C_{Q}=470 \mathrm{pF}$. Vertical scales are different for clarity. 

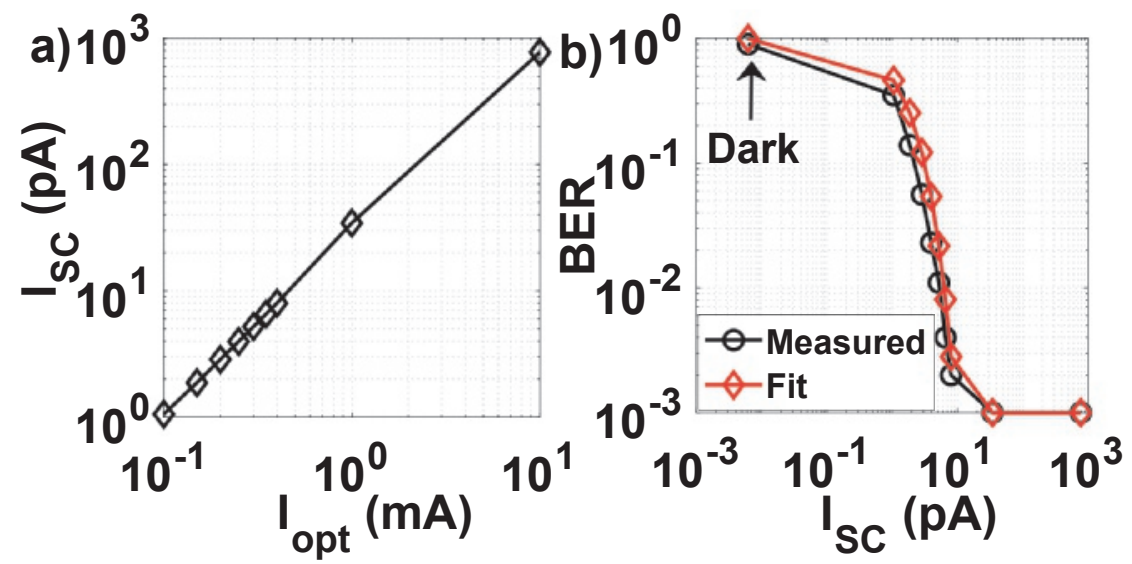

Figure 4.14: a) Measured short circuit current $\left(I_{\mathrm{SC}}\right)$ in response to current in the external illumination source $\left(I_{\text {opt }}\right)$. (b) Measured and fitted BER as a function of $I_{\mathrm{SC}}$. All axes are on a log scale.

current $\left(I_{\mathrm{SC}}\right)$ of J12 as a function of the external LED current $\left(I_{\text {opt }}\right)$. $I_{\mathrm{SC}}$ is the measured current through the AMLED at $V_{\mathrm{R}}=0$. For higher $I_{\mathrm{opt}}$, more carriers are generated optically and therefore $I_{S C}$ increases [12]. This implies a higher $N_{\text {opt }}$ and therefore a higher $N_{\mathrm{d}}$ (Eq. 4.4(b)).

Fig. 4.14(b) shows the measured BER as a function of $I_{\mathrm{SC}}$ when the driver circuit was operated at $V_{\mathrm{BIAS}}=17.6 \mathrm{~V}, V_{\mathrm{DD}}=6 \mathrm{~V}$ and $C_{\mathrm{Q}}=470 \mathrm{pF}$. For comparison, Eq. $(4.4 \mathrm{~d})$ is also plotted with $N_{\mathrm{d}} \approx I_{\mathrm{SC}} T_{\mathrm{ON}} / q_{\mathrm{e}}$ and a fitting parameter $\eta=7 \times 10^{12}$, showing good agreement with the measurements.

The corresponding jitter PDFs in Fig. 4.15 demonstrate a lower standard deviation for higher $I_{\mathrm{SC}}$, also because of a higher $N_{\mathrm{d}}$. The standard deviation of the jitter was estimated to be $\sim 10 \mathrm{~ns}$ at $I_{\mathrm{SC}}=774.5 \mathrm{pA}$.
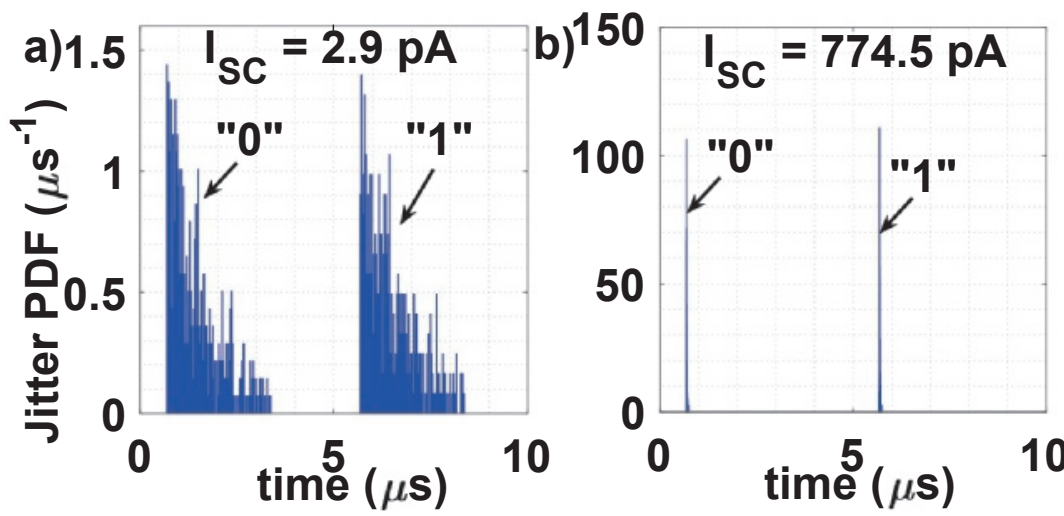

Figure 4.15: Example of measured PDF of jitter for " 0 " and " 1 " at two $I_{\mathrm{SC}}$ settings. The driver circuit was operated at the same bias settings as in Fig. 4.14(b). Y-axis scales are different for clarity. 


\subsubsection{Impact on light output}

For an optical link, the photon transmission to a nearby photodetector is important. We have already shown a linear relation between $Q_{b}$ and the emitted photon flux from an AMLED [13, 71]. Therefore, triggering of an avalanche during a data bit impacts the light emission from an AMLED for that bit $[71,111]$.

A high BER in the transmitted data implies that light would not be emitted from an AMLED during the data bit transfer. Assuming an ideal receiver, this would result in a high BER of the overall optical link, which is obviously undesired.

Furthermore, a high jitter in the turn-on time of an avalanche would cause a high jitter in the light output from an AMLED. This would limit the application of AMLEDs in optical links using time critical modulation schemes, such as PPM [109].

\subsection{Design recommendations}

From the results in section 4.3, we can conclude that jitter and BER in AMLEDs can be reduced through some design techniques. We discuss some of the design recommendations for high speed AMLEDs.

\subsubsection{Effect of defect density}

By the use of electron beam induced scanning electron microscopy it was shown [112] that avalanche is favored at the defect sites, indicating that defect locations are suitable for the triggering of avalanche. In modern CMOS technologies, the defect density can be increased by increasing the proximity of the depletion region to oxide interfaces, such as shallow trench isolation (STI) [77].

To demonstrate the effect of STI, a $\mathrm{p}^{+} \mathrm{n}$ AMLED with an STI interface and another device without an STI interface were selected from a $140 \mathrm{~nm}$ SOI CMOS technology. Fig. 4.16(a) shows the schematic cross-sections for these devices, denoted as S7 and C12 [79]. S7 is square in shape with an edge length of $7 \mu \mathrm{m}$, whereas C12 is a circular device with a diameter of 12 $\mu \mathrm{m}$.

Fig. 4.16(b) shows the $I-V$ characteristics for these AMLEDs. The different $V_{\mathrm{BR}}$ for S7 and C12 can be attributed to the different doping of the n-layer in these devices. Assuming one-sided abrupt junctions, the n-well doping in S7 and C12 is estimated as $6 \times 10^{16}$ and $9 \times 10^{16} \mathrm{~cm}^{-3}$ respectively [12]. Fig. 4.16(b) shows an example of the light emission from these devices. The PPM speed of these AMLEDs was measured using the same driver circuit and PRBS data (Fig. 4.6). Figs. 4.16(c) and 4.16(d) show examples of the measured transients at the indicated bias settings. As the $V_{\mathrm{EX}, \mathrm{i}}$ at these bias settings is almost same $(\sim 4.9 \mathrm{~V})$, the difference in the peak current of the transients can be explained by the lower resistance of 
C12 due to a higher n-well doping, combined with geometric effects [12]. Figs. 4.16(e) and 4.16(f) show examples of the measured PDFs of jitter in S7 and $\mathrm{C} 12$ respectively.

For S7, the minimum attainable BER of $10^{-3}$ (section 4.3) and a standard deviation of jitter of $\sim 37 \mathrm{~ns}$ were measured. The measured energy-per-bit at a data rate of $100 \mathrm{kbps}$ was $\sim 23.8 \mathrm{~nJ} /$ bit. In comparison, C12 showed a higher BER and jitter. These results demonstrate that an STI interface at the junction can help AMLEDs to achieve a higher speed.

\subsubsection{Other recommendations}

A three terminal AMLED structure was proposed and demonstrated [15] to increase its quantum efficiency. The basic idea is that the third terminal (emitter) injects more carriers, hence higher $N_{\mathrm{d}}$, in the depletion region thereby triggering avalanche, which results in a low BER and jitter. Consequently, such a device concept results in high speed.

Another so-called $\mathrm{n}^{+} \mathrm{pn}^{+} \mathrm{pn}^{+}$device structure has been proposed to increase the efficiency of an AMLED [113]. This structure comprises two reverse biased light-emitting junctions and two forward-biased junctions to increase intra-band transitions by providing extra carriers. Such a device structure could also improve the speed.

Ideally, for a high speed AMLED, the maximum attainable speed is only limited by the time constants of the driver circuit (and not by triggering speed of avalanche in AMLEDs), which can be in the range of tens of picoseconds in modern CMOS technologies. This could result in a maximum attainable speed in the Gbps range. Furthermore, the ultimate achievable speed is limited by the small-signal modulation speed, which has been shown to be in the range of tens of $\mathrm{GHz}[63,107]$.

\subsection{Conclusions}

The data transmission capabilities of silicon avalanche mode LEDs (AMLEDs) fabricated in CMOS was investigated. The data were modulated using pulse position modulation (PPM) and the bit-error-rate (BER) and the jitter in the transmitted data were measured. The results were correlated to the multiplication noise $\left(\sigma_{M}\right)$ and the leakage current of the AMLEDs. The $\sigma_{M}$ of these AMLEDs increases with their size because of more defects in larger devices. It was shown that AMLEDs with a higher $\sigma_{M}$ display a lower BER. Through temperature and optical excitations, it was shown that the leakage current also improves the triggering of avalanche, thereby reducing BER and jitter. A PPM speed of $100 \mathrm{kbps}$ at the energy consumption of $23.8 \mathrm{~nJ} /$ bit has been obtained from the high-speed AMLEDs. The triggering rate can be improved using AMLED designs to inject more carriers in the depletion region and some recommendations for such high speed 
AMLED designs were proposed. The presented results are important for the design of low power monolithic optical links. 

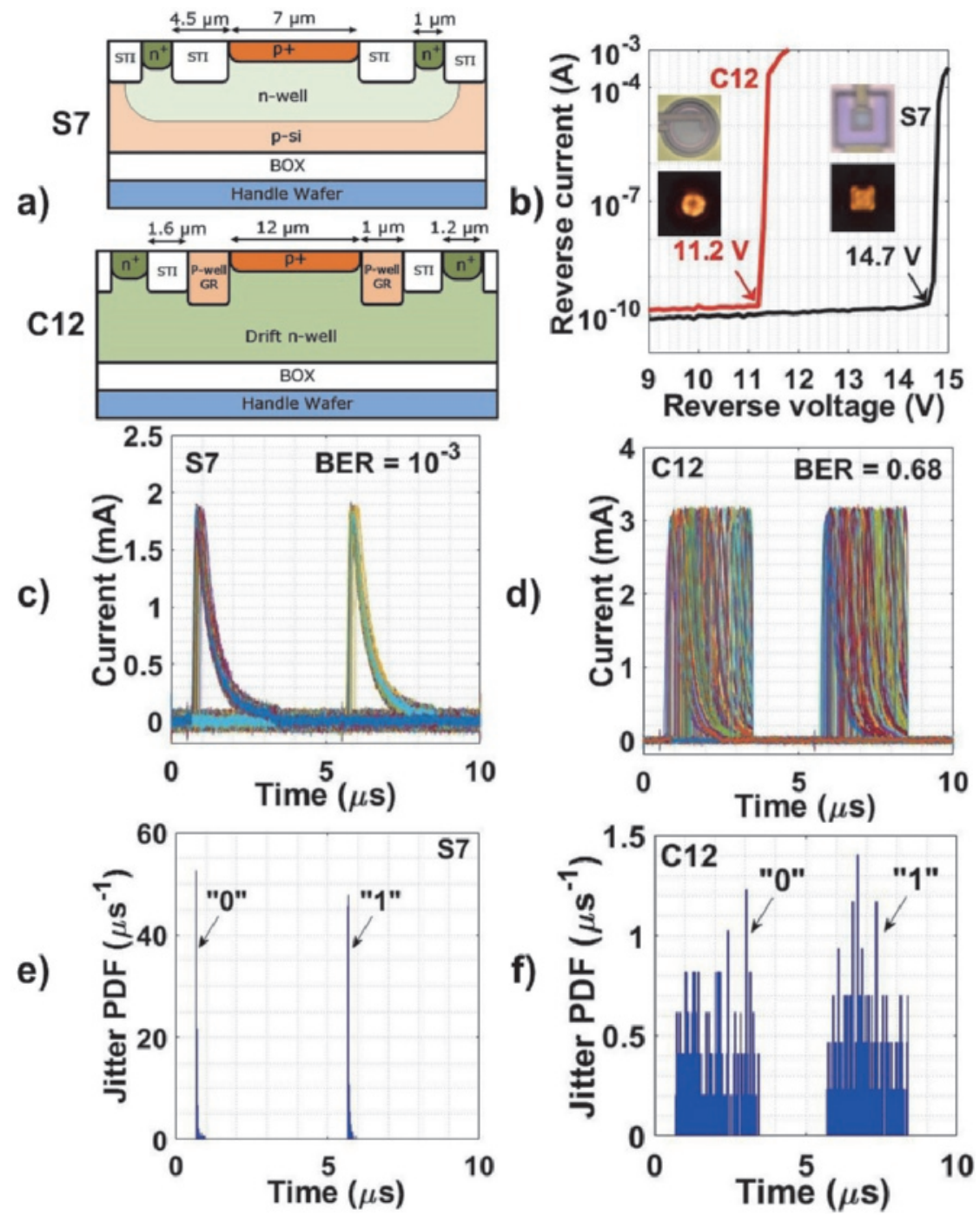

Figure 4.16: (a) Schematic cross-section of a square shaped AMLED (S7) and a circular AMLED (C12) in a $140 \mathrm{~nm}$ SOI CMOS technology. (b) Reverse I - V characteristics measured using a Keysight B2901A SMU with $1 \mathrm{~s}$ integration time. The light emission from these AMLEDs, biased at a reverse current of $1 \mathrm{~mA}$, is also shown. The emission was captured using a Nikon D3100 camera with an integration time of 30 s. (c) An example of the measured transients for $\mathrm{S} 7$ for $2^{10}$ bits when the driver circuit was operated with $V_{\text {BIAS }}=19.6 \mathrm{~V}, V_{\mathrm{DD}}=5 \mathrm{~V}$ and $\mathrm{C}_{\mathrm{Q}}=470 \mathrm{pF}$. (d) Measured transients for $\mathrm{C} 12$ at $V_{\mathrm{BIAS}}=16.1 \mathrm{~V}, V_{\mathrm{DD}}=5 \mathrm{~V}$ and $\mathrm{C}_{\mathrm{Q}}=470 \mathrm{pF}$. $(\mathrm{e}, \mathrm{f})$ Measured PDFs of jitter for bit " 0 " and bit " 1 " at the same bias settings for the same devices. 


\title{
OPTICAL TRANSMITTER USING AVALANCHE LEDS IN SOI CMOS TECHNOLOGY
}

\begin{abstract}
This chapter presents a low power monolithically integrated optical transmitter with avalanche mode light emitting diodes (AMLEDs) in a $140 \mathrm{~nm}$ silicon-on-insulator CMOS technology. Silicon AMLEDs exhibit wide-spectrum electroluminescence (400 $\mathrm{nm}<\lambda<850 \mathrm{~nm}$ ), which has a significant overlap with the responsivity of silicon photodiodes. This enables monolithic CMOS integration of optocouplers, for e.g. smart power applications requiring high data rate communication with a large galvanic isolation. To ensure a certain minimum number of photons per data pulse (or per bit), LED drivers must be robust against process, operating conditions and temperature variations of the LED. Combined with the AMLED's steep current-voltage curve at relatively high breakdown voltages, this conventionally results in high power consumption and significant heating. The presented transmitter circuit is intrinsically robust against these issues, thereby enabling low power operation.
\end{abstract}

This chapter is based on a publication in Optics Express [71]. 


\subsection{Introduction}

Many smart power applications require data communication with galvanic isolation. Currently this is achieved with inductive isolators (transformers), capacitive isolators or discrete optocouplers [20]. Integrated transformers are big and significantly add to cost and size while they are also prone to external electro-magnetic interference (EMI) [20]. Capacitive isolators can be integrated in the backend, but are relatively big when isolating between voltage domains that have a large voltage difference. Optocouplers on the other hand are immune to EMI effects and monolithic integration of optocouplers is attractive for smart power and on chip communication applications [38].

Wide spectrum electroluminescence (EL) from silicon (Si) p-n junctions operating in avalanche mode has been reported earlier [14, 114, 115]. Avalanche mode light emitting diodes (AMLEDs) are fast with reported small signal modulation speed in the range of tens of GHz [63]. An AMLED as a light source in a CMOS integrated optocoupler has also been proposed $[16,17,41,115,116]$. The coupling efficiency between the AMLED and an Si PD has been reported to be higher as compared to the same LED in forward mode of operation $[13,116]$. This is because of the stronger overlap between the emission spectrum of Si AMLEDs and the spectral responsitivity of Si PDs [17].

For optocoupling applications with a sufficiently low bit-error-rate (BER), the AMLED driver must ensure a certain minimum number of photons at the receiver side for data communication. However, optoelectronic properties of AMLEDs are sensitive to process, voltage and temperature (PVT) variations [65]. Together with their steep current-voltage (I-V) curve at relatively high voltages, this easily results in high power consumption, and significant heating $[13,116]$ which are bottlenecks to implement power efficient On-Off Keying (OOK) LED driver circuits in optocoupling applications. In this chapter, an AMLED driver circuit is introduced to solve these issues, enabling low power Si integrated optical transmitters.

Section 5.2 of this chapter describes several physics related properties of the AMLED that are relevant for this work. An estimate of the transmission efficiency of the designed optical link is estimated in section 5.3 which is essential to characterize the AMLED in terms of its photon flux output. The principle of the driver circuit is to drive the AMLED (per data bit) with a minimum quantity of avalanche charge required to get certain amount of detectable photons at the PD, independent of PVT variations. The circuit to implement these features is introduced in section 5.4. The robustness, low power consumption and emission properties of the AMLED integrated with the driver circuit are demonstrated using the measurement results in section 5.5. Section 5.6 discusses the potential of using the AMLED with the introduced driver circuit in a monolithically integrated CMOS optocoupler. This chapter is concluded in section 5.7. 


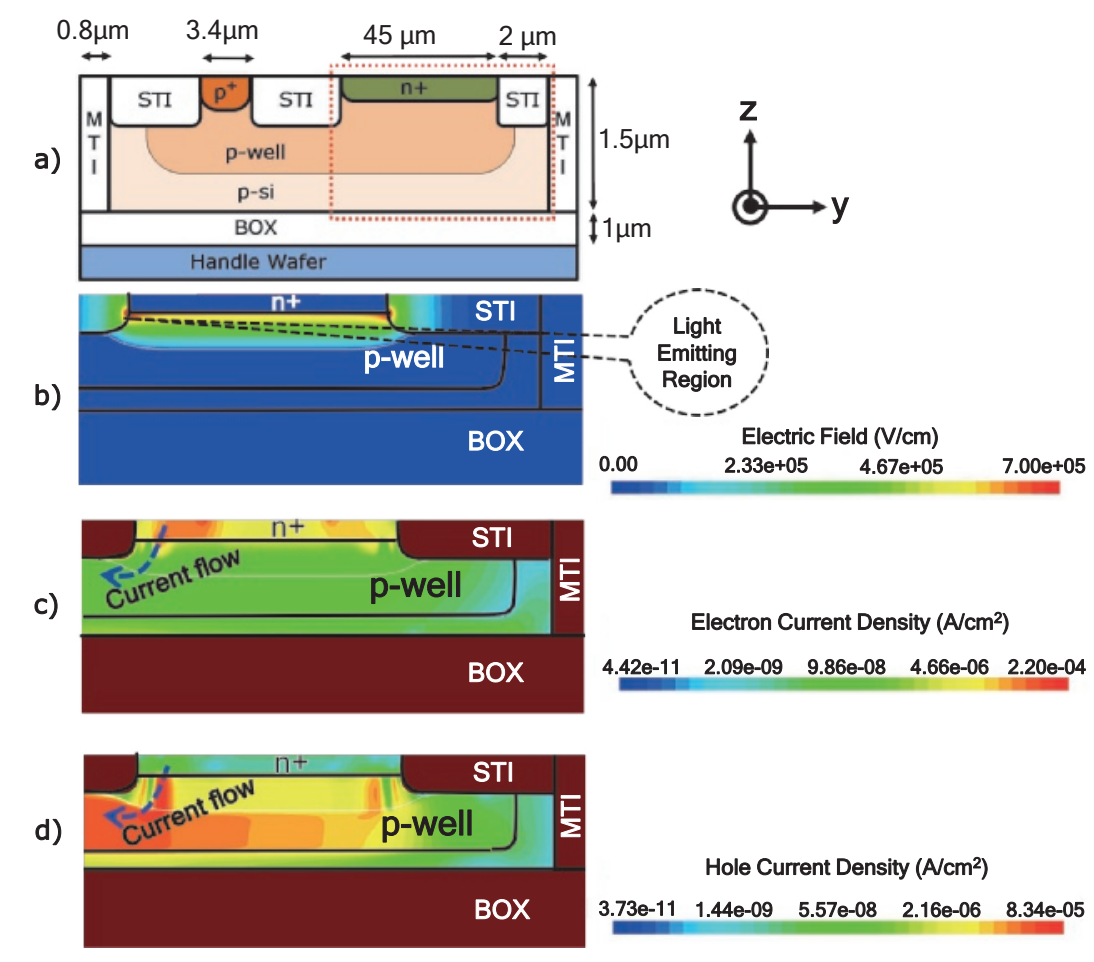

Figure 5.1: (a) A schematic cross-section of the $n^{+}$-pwell AMLED (not to scale). (b) TCAD simulated electric field of the highlighted region in (a) at $V_{\text {BIAS }}=18 \mathrm{~V}$; light is emitted from the indicated region. (c) TCAD simulated electron current density. (d) TCAD simulated hole current density.

\subsection{Optoelectronic properties of the AMLED}

Fig. 5.1(a) shows a schematic cross-section of an AMLED (not to scale) in a $140 \mathrm{~nm}$ SOI CMOS technology [36]. The Medium Trench Isolation (MTI) regions and Buried Oxide (BOX) layer isolate the high voltage at the AMLED from the CMOS circuitry and provide galvanic isolation from the receiver. Fig. 5.1(b) shows the TCAD simulated 2-D electric field profile (in the $y-z$ plane), for the regions in the dashed box in Fig. 5.1(a). The field was simulated above breakdown: at a reverse bias ( $\left.V_{\text {BIAS }}\right)$ of $18 \mathrm{~V}$ for an AMLED having a breakdown voltage $\left(V_{\mathrm{BR}}\right)$ of $\sim 17 \mathrm{~V}$. Avalanche breakdown and hence avalanche mode light emission is initiated in the region with the highest electric field [117], indicated in Fig. 5.1(b). Further, light is emitted mainly from the $\mathrm{n}^{+}$periphery that is closest to the $\mathrm{p}^{+}$ contact (along x-axis) in Fig. 5.1(b). Figs. 5.1(c)-5.1(d) show the TCAD simulated electron and the hole current density for the dashed region in Fig. 5.1(a).

A schematic top view of the AMLED and the integrated PD, including their dimensions is shown in Fig. 5.2(a). Two identical diodes have been 
a)

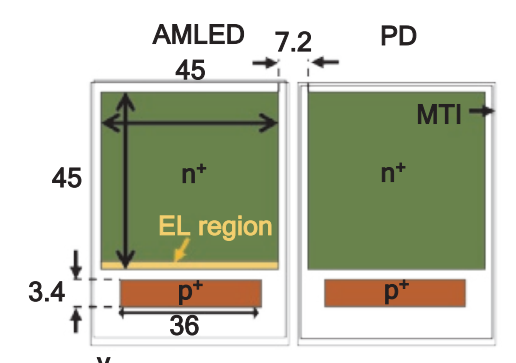

c)

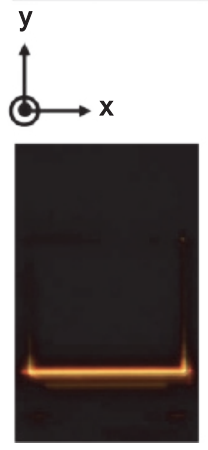

AMLED

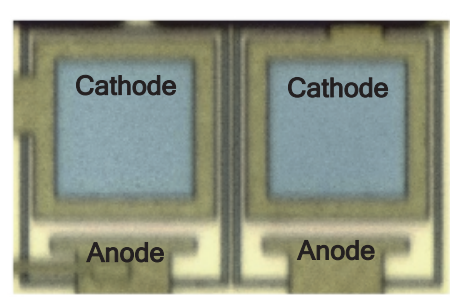

d)

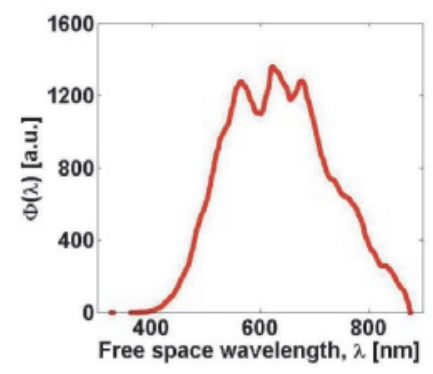

Figure 5.2: (a) Schematic top view of the AMLED and the PD which are basically identical diodes; all dimensions are in $\mu \mathrm{m}$. (b) Micrograph of the designed AMLED-PD structure. (c) Micrograph of the light emission from the AMLED using $25 \mathrm{~s}$ camera integration time, biased at a $4 \mathrm{~mA} \mathrm{DC}$ current using an Agilent B2901A. (d) Emitted photon spectral flux density $(\Phi(\lambda))$ at $T=25^{\circ} \mathrm{C}$; measured vertically using an Avantes ADC-1000-USB spectrometer and an integration time of $30 \mathrm{~s}$. The arbitrary units [a.u.] are because of the unknown capture efficiency of the spectrometer.

used, one acting as an AMLED and the other acting as a PD. Fig. 5.2(b) shows the micrograph of the AMLED and PD. This PD is only used to measure photon flux of the AMLED as discussed in section 5.5.2. Fig. 5.2(c) shows an EL-micrograph of the AMLED demonstrating emission at one side (along the $x$-axis indicated in Fig. 5.2(a)) as explained by TCAD simulation results in Fig. 5.1(b)-5.1(d). Fig. 5.2(d) shows the emission spectrum of the AMLED as measured vertically. Fabry-perot interference in the back-end causes the ripples in this vertical emission spectrum.

Measured reverse $I-V$ characteristics of the AMLED are shown in Fig. 5.3(a); for circuit simulations, these characteristics were used in a tablebased (interpolated) $I-V$ model combined with a junction capacitance model. $V_{\mathrm{BR}}$, as indicated in Fig. 5.3(a), is defined as the voltage at which the $I_{\text {AMLED }}$ starts to sharply increase. Fig. 5.3(b) shows a linear relation between the AMLED avalanche charge $\left(Q_{\text {AMLED }}\right)$ and the vertically emitted number of photons as calculated by Eq. (5.2) (section 5.3). For Fig. 5.3(b), the AMLED was biased at several DC currents ( $\left.I_{\text {AMLED }}\right)$ using an Agilent B2901A source and measurement unit (SMU) (with measurement integration time $=1 \mathrm{~s}$ ) and the vertical emission spectrum was measured 

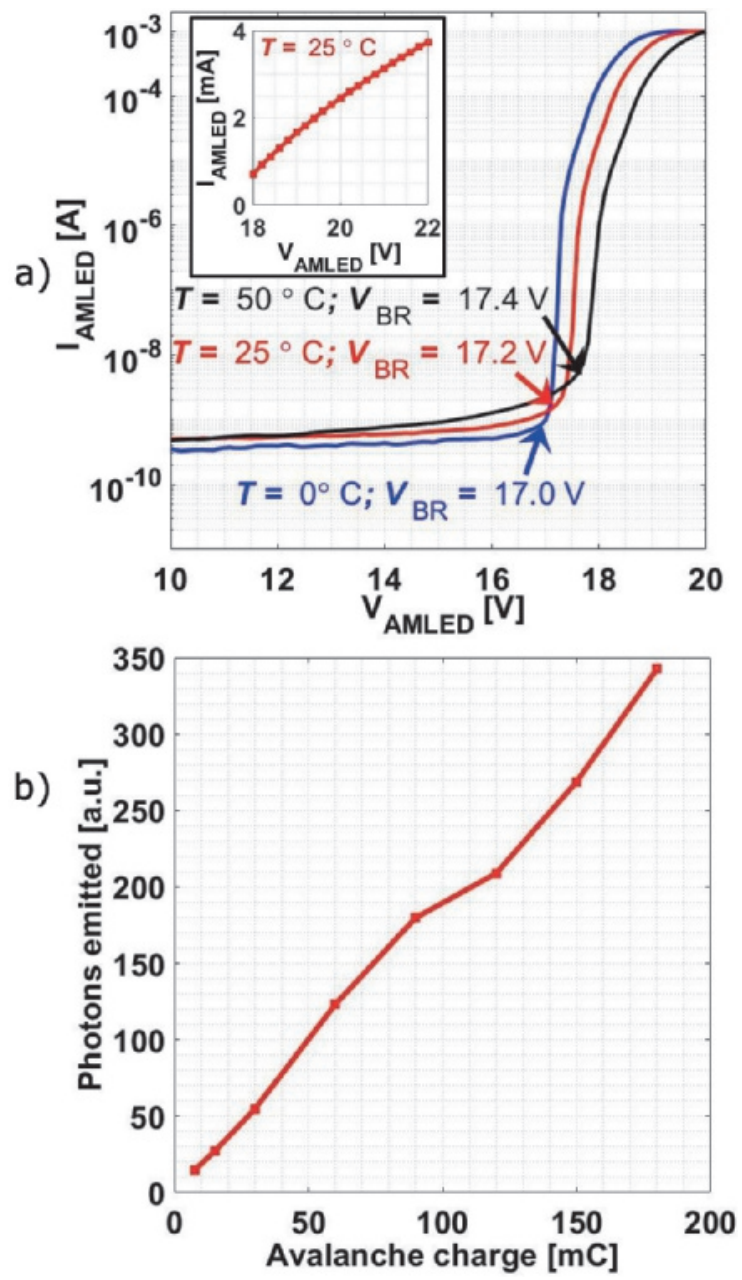

Figure 5.3: (a) Measured AMLED reverse $I-V$ characteristics at several temperatures using an Agilent B2901A and with 1s integration time. The inset shows the $I-V$ characteristics (on linear scale) in the breakdown region to explicitly show the resistance $(\sim 1.4 \mathrm{k} \Omega)$ of the AMLED at $T=25^{\circ} \mathrm{C}$. (b) Estimated total number of vertically emitted photons (arbitrary units) as a function of AMLED charge $\left(Q_{\text {AMLED }}\right)$ at $T=25^{\circ} \mathrm{C}$.

using an Avantes ADC-1000-USB spectrometer with measurement integration time ( $\left.T_{\text {integration }}\right)$ of $30 \mathrm{~s}$ at each setting. $Q_{\text {AMLED }}$ is calculated as $Q_{\text {AMLED }}=\int_{0}^{T_{\text {integration }}} I_{\text {AMLED }}(\mathrm{t}) \mathrm{d} \mathrm{t}$.

\subsection{Optical link transmission efficiency, $\eta_{\mathrm{TE}}$}

From a system level perspective, the total number of photons received at the PD per bit $\left(N_{\text {photons, }, \mathrm{PD}}\right)$ in response to the electrical energy spent in the AMLED per bit $\left(E_{\mathrm{b}}\right)$ is important. As discussed in section 5.5.2, $E_{\mathrm{b}}$ is pro- 


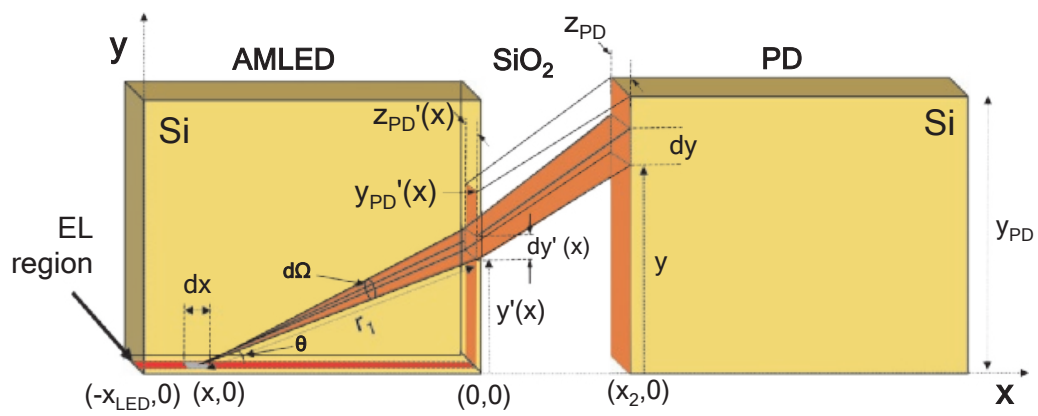

Figure 5.4: Schematics of the AMLED-PD to estimate the optical transmission efficiency $\left(\eta_{\mathrm{TE}}\right)$. For clarity, important dimensions are enlarged. $x_{\mathrm{LED}}$ is the width of the AMLED $(=45 \mu \mathrm{m}), x_{2}(=7.2 \mu \mathrm{m})$ is the separation between the AMLED and the PD and $z_{\mathrm{PD}}=1.5 \mu \mathrm{m}$ is the thickness of the SOI layer.

portional to the number of electrons flowing through the AMLED per bit ( $\left.N_{\text {electrons,AMLED }}\right)$. For estimating $N_{\text {photons,PD }}$ in response to $N_{\text {electrons,AMLED, }}$ the total coupling quantum efficiency of the link $\left(\eta_{\text {system }}\right)$ should be determined, that is defined as $\eta_{\text {system }}=\frac{N_{\text {photon, }, \mathrm{PD}}}{N_{\text {electron, AMLED }}} \propto \frac{N_{\text {photons }, \text { PD }}}{E_{\mathrm{b}}}$. This $\eta_{\text {system }}$ can be written as a product of two efficiencies. The first one is the internal quantum efficiency (IQE) of the AMLED which relates the number of photons emitted per bit from the AMLED ( $N_{\text {photons,AMLED }}$ ) to $N_{\text {electrons,AMLED }}$ [21]. The second efficiency component is the transmission efficiency of the optical link between the AMLED and the PD, denoted as $\eta_{\mathrm{TE}}$.

$$
\begin{aligned}
\eta_{\text {system }} & =I Q E \cdot \eta_{\text {TE }} \\
& =\frac{N_{\text {photons,AMLED }}}{N_{\text {electrons,AMLED }}} \cdot \frac{N_{\text {photons,PD }}}{N_{\text {photons,AMLED }}} .
\end{aligned}
$$

The IQE is estimated from DC measurements in section 5.5.2. In this section, the $\eta_{T E}$ is estimated. Fig. 5.4 represents the schematic structure of the design. The following steps describe the $\eta_{\mathrm{TE}}$ estimation procedure:

1. The total number of photons emitted by the AMLED ( $\left.N_{\text {photons,AMLED }}\right)$ is calculated. As shown in Fig. 5.2(c), light emission occurs predominantly at the lower edge of the AMLED. This light emitting region is modeled as a line of length $x_{\mathrm{LED}}$, emitting uniformly and isotropically $[118,119]$. Total number of photons emitted by the AMLED ( $\left.N_{\text {photons,AMLED }}\right)$ is given by:

$$
N_{\text {photons,AMLED }}=\int_{\lambda} \Phi(\lambda) \mathrm{d} \lambda,
$$

where $\Phi(\lambda)$ is the photon spectral flux density, shown in Fig. 5.2(d).

2. The total number of photons received at the PD ( $\left.N_{\text {photons,PD }}\right)$ is calculated; it is assumed that $N_{\text {photons,PD }}$ is limited only by [12]: 
- absorption losses in the AMLED. The absorption coefficient $(\alpha)$ is $\lambda$ dependent and for this layout of the AMLED and the PD (as shown in Fig. 5.2(a)), most of the photons emitted at short $\lambda$ are absorbed in the AMLED itself before reaching the PD.

- the finite solid angle of the PD over the AMLED. The solid angle is limited either by the critical angle at the $\mathrm{Si}-\mathrm{SiO}_{2}$ interface or by the (apparent) size of the PD (Fig. 5.4). Due to refraction at the $\mathrm{Si}-\mathrm{SiO}_{2}$ interface, the apparent height of the PD seen at each point along the $x$-axis of the AMLED is different: the apparent height of the PD $\left(y_{\mathrm{PD}}\right)$ is reduced to $y_{\mathrm{PD}}^{\prime}(x)$. Similarly, the width of the PD $\left(z_{\mathrm{PD}}\right)$ is reduced to $z_{\mathrm{PD}}^{\prime}(x)$.

- the transmittance at the $\mathrm{Si}-\mathrm{SiO}_{2}$ interfaces $\left(T_{X}(x, y)\right)$, which is given by Fresnel's equations [21]. To simplify calculations, constant $(\lambda$-invariant) refractive indices $(n)$ have been used for $\mathrm{Si}$ $(n=3.9)$ and $\mathrm{SiO}_{2}(n=1.5)$.

Along the $x$-direction, the lateral dimensions of the PD are much larger than the absorption length of the photons in the spectral region of interest. Therefore it is assumed that all the photons that reach the PD are detected.

Along the $z$-direction, for the DC measurements (section 5.5.2), any photon absorbed within a diffusion length from the depletion edge of the PD will contribute to the photocurrent [12].

Under these assumptions and using Fig. 5.4, the received photon spectral flux density per unit length at the PD is:

$$
\begin{aligned}
\Phi_{\mathrm{PD}}(x, y, \lambda)=\Phi_{\mathrm{LED}}(\lambda) \cdot \exp \left(-\alpha(\lambda) \cdot r_{1}\left(x, y^{\prime}(x)\right)\right) & \\
& T_{\mathrm{X}}\left(0, y^{\prime}(x)\right) \cdot T_{X}\left(x_{2}, y\right) \cdot \Omega(x, y),
\end{aligned}
$$

where:

- $\Phi_{\mathrm{LED}}(\lambda)=\frac{\Phi(\lambda)}{x_{\mathrm{LED}}}$ is the emitted photon spectral density per unit length.

- $r_{1}\left(x, y^{\prime}(x)\right)=\sqrt{x^{2}+\left(y^{\prime}(x)\right)^{2}}$ is the distance travelled by photons emitted at $(x, 0)$ within the AMLED, while propagating towards $\left(x_{2}, y\right)$.

- $T_{X}\left(0, y^{\prime}(x)\right)$ is the transmittance of the AMLED-SiO ${ }_{2}$ interface and $T_{X}\left(x_{2}, y\right)$ is the transmittance of the $\mathrm{SiO}_{2}-\mathrm{PD}$ interface.

- $\Omega(x, y)=\frac{\mathrm{d} \Omega}{\mathrm{d} y^{\prime}}=\frac{z_{\mathrm{PD}}^{\prime}(x) \cdot \cos (\theta)}{4 \pi r_{1}^{2}\left(x, y^{\prime}(x)\right)}$ is the solid angle per unit length subtended by the PD over the $\mathrm{d} x$ section of the AMLED.

The total number of photons received at the PD because of emission from the entire light emitting area of the AMLED ( $\left.N_{\text {photons,PD }}\right)$ is given 
by:

$$
N_{\text {photons }, \mathrm{PD}}=\int_{0}^{-\chi_{\mathrm{LED}}} \int_{0}^{y_{\mathrm{PD}}^{\prime}(x)} \int_{\lambda} \Phi_{\mathrm{PD}}(x, y, \lambda) \mathrm{d} \lambda \mathrm{d} y^{\prime} \mathrm{d} x
$$

3. Using numerical integration in Eq. (5.4), the $\eta_{\mathrm{TE}}=\left(\frac{N_{\text {photons,PD }}}{N_{\text {photons, AMLED }}}\right)$ of the link is estimated as $3 \times 10^{-4}$.

It is emphasized that the $\eta_{\mathrm{TE}}$ of this link is low due to mainly the suboptimum (side-by-side) layout of the AMLED and the PD, as shown in Fig. 5.2(a) and 5.2(b). A possible direction to improve $\eta_{\mathrm{TE}}$ is discussed in section 5.6.

\subsection{AMLED driver circuit for an optocoupler}

The main idea of the circuit is to drive the AMLED with a minimum amount of avalanche charge per data bit $\left(Q_{\mathrm{b}}\right)$, required to get a certain amount of photons at the PD, independent of PVT variations, with a relatively small area and relatively low demands on the driver circuit (including timing demands).

Fig. 5.5 shows the principle of the self-quenched AMLED driver circuit (using idealized time domain waveforms) that limits the $Q_{\mathrm{b}}$ by dynamically quenching the avalanche process. The voltage across the AMLED is denoted as $V_{\text {AMLED }}\left(=V_{\text {BIAS }}-V_{\text {CAP }}\right)$. The excess bias voltage across the AMLED $\left(V_{\mathrm{EX}}\right)$ is defined as the extra voltage above $V_{\mathrm{BR}}$ [22], $V_{\mathrm{EX}}=V_{\mathrm{AMLED}}-V_{\mathrm{BR}}$. The current through the AMLED is denoted as $I_{\mathrm{AMLED}}$ and the series resistance of the AMLED for $V_{\mathrm{AMLED}}>V_{\mathrm{BR}}$ as $R_{\text {AMLED }} \cdot R_{\text {AMLED }}$ is estimated from the measured $I-V$ characteristics of Fig. 5.3(a) and is assumed constant for $V_{\text {AMLED }}>V_{\text {BR }}$ for simplicity [22]. The junction capacitance of the AMLED in and near avalanche is modeled by capacitance $C_{\text {AMLED }}$. The resistance of the driver circuit is negligible in comparison to $R_{\mathrm{AMLED}}$ and hence is ignored. A simplified model for the AMLED is also shown in Fig. 5.5 including $C_{\text {AMLED }}$ [22]. For this section, it is assumed that $C_{\mathrm{AMLED}}<<C_{\mathrm{Q}}$ for simplicity reasons.

The operating principle is described using Fig. 5.5. Initially, the voltage across the $C_{\mathrm{Q}}, V_{\mathrm{CAP}}-V_{\mathrm{DRV}}$ is 0 . First reset switch $\mathrm{M} 1$ is opened (using control signal RST) after which the input (IN) is set high at $t=0$. As IN is set high, the $V_{\mathrm{DRV}}$ becomes low and instantly $V_{\mathrm{CAP}}$ also becomes low, the initial $V_{\text {AMLED }}=V_{\text {BIAS }}-V_{\text {CAP }} \approx V_{\text {BIAS }}$ and $V_{\text {EX }}=V_{\text {BIAS }}-V_{\text {BR }}$ (assuming $\left.C_{\text {AMLED }}<<C_{\mathrm{Q}}\right)$. The initial $V_{\mathrm{EX}}$ yields an initial $I_{\mathrm{AMLED}}=V_{\mathrm{EX}} / R_{\mathrm{AMLED}}$.

After the AMLED goes into the avalanche, $I_{\mathrm{AMLED}}(t)$ charges the quenching capacitor $C_{Q}$ which results in approximately an exponentially increasing $V_{\mathrm{CAP}}$, hence in approximately an exponentially decreasing $V_{\mathrm{AMLED}}$ and $V_{\mathrm{EX}}$. As $V_{\mathrm{AMLED}}$ approaches $V_{\mathrm{BR}}$, the avalanche is quenched.

For $\mathrm{t}>T_{\mathrm{ON}}$, first IN is set low turning the driver off and then M1 is closed to reset the $V_{\mathrm{CAP}}$ to $V_{\mathrm{DD}}$. Since the avalanche quenches itself by 


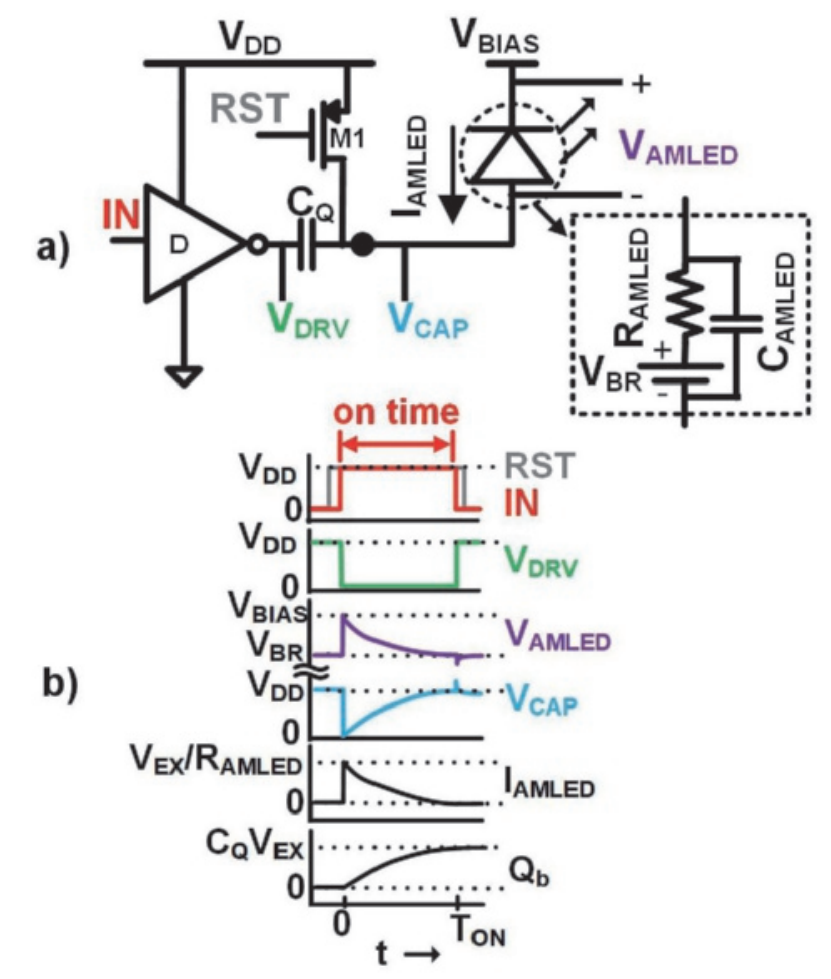

Figure 5.5: a) Principle of the self-quenched driver circuit. For these AMLEDs, $V_{\text {BIAS }}>>V_{\text {DD }}$. A simplified model for the AMLED is shown in the dashed area [22]. b) Idealized time domain waveforms.

reducing $V_{\mathrm{AMLED}}$ to $V_{\mathrm{BR}}$, this circuit is denoted as a self-quenched driver circuit.

$V_{\mathrm{AMLED}}(\mathrm{t}), V_{\mathrm{EX}}(\mathrm{t}), I_{\mathrm{AMLED}}(\mathrm{t})$ and $Q_{\mathrm{b}}(\mathrm{t})$ can now be estimated: assuming the total resistance to be $R_{\mathrm{AMLED}}$ and the total capacitance to be $C_{\mathrm{Q}}$, Eqs. (5.5) describe the mentioned physical quantities for $0 \leqslant t \leqslant T_{\mathrm{ON}}$. The initial $V_{\mathrm{EX}}$ has been denoted as $V_{\mathrm{EX}, 0}$ :

$$
\begin{gathered}
V_{\mathrm{AMLED}}(\mathrm{t})=V_{\mathrm{BIAS}}-V_{\mathrm{EX}, 0} \cdot\left(1-\exp \left(-\frac{t}{R_{\mathrm{AMLED}} C_{\mathrm{Q}}}\right)\right) \\
V_{\mathrm{EX}}(\mathrm{t})=V_{\mathrm{EX}, 0} \cdot \exp \left(-\frac{t}{R_{\mathrm{AMLED}} C_{\mathrm{Q}}}\right) \\
I_{\mathrm{AMLED}}(t)=\frac{V_{\mathrm{EX}, 0}}{R_{\mathrm{AMLED}}} \cdot \exp \left(-\frac{t}{R_{\mathrm{AMLED}} C_{\mathrm{Q}}}\right) \\
Q_{\mathrm{b}}=\int_{0}^{T_{\mathrm{ON}}} I_{\mathrm{AMLED}}(t) \mathrm{d} \mathrm{t}=C_{\mathrm{Q}} V_{\mathrm{EX}, 0} \cdot\left(1-\exp \left(-\frac{T_{\mathrm{ON}}}{R_{\mathrm{AMLED}} C_{\mathrm{Q}}}\right)\right) \leqslant C_{\mathrm{Q}} V_{\mathrm{EX}, 0} .
\end{gathered}
$$


Eq. (5.5d) shows that $Q_{\mathrm{b}}$ is limited to $C_{\mathrm{Q}} V_{\mathrm{EX}, 0}\left(\leqslant \mathrm{C}_{\mathrm{Q}} V_{\mathrm{DD}}\right)$.

The complete schematics of the implemented circuit are shown in Fig. 5.6(a). The driver circuit comprises a chain of inverters (with enable functionality to switch $C_{Q}$ ) and a reset transistor. Selecting a $C_{Q}$ value is achieved using a number of parallel identical drivers (D1 to D7) connected to the AMLED anode which can be controlled using their respective enable (EN) signals. Using D8 (identical to D1 to D7), the AMLED can be operated without any quenching (Fig. 5.10(c)-(d)). To enable the measurement of fast AMLED current transients at the onset of the avalanche, a differential structure is adopted and a measurement circuit was implemented using an open drain PMOS differential amplifier [9]. The measurement setup enables us to measure at frequencies upto $2.8 \mathrm{GHz}$. A micrograph of the implemented chip in a $140 \mathrm{~nm}$ CMOS SOI technology is shown in Fig. 5.6(b) [36]. It is shown that this driver circuit is robust to many physics issues related to the power dissipation and PVT variations of an AMLED.

\subsection{Measurement results}

In this section, to demonstrate the functionality of the circuit, an example of the measured transient avalanche current ( $I_{\text {AMLED }}$ ) waveform is shown. Further, the measured energy-per-bit $\left(E_{\mathrm{b}}\right)$ of the presented transmitter and its photon flux output with respect to $E_{\mathrm{b}}$ is presented. The robustness of $E_{\mathrm{b}}$ against process, voltage, temperature, design and pulse width variations is then demonstrated.

\subsubsection{Functionality}

With an off-state $V_{\mathrm{AMLED}} \approx V_{\mathrm{BR}}$, in the on-state the AMLED is driven to $V_{\mathrm{AMLED}} \approx V_{\mathrm{BR}}+V_{\mathrm{EX}, 0}$ where $V_{\mathrm{EX}, 0}$ is $V_{\mathrm{DD}}$ divided between $C_{\mathrm{Q}}$ and $C_{\mathrm{AMLED}}$ at $t=0$ (using the simplified model of the AMLED shown in Fig. 5.5):

$$
V_{\mathrm{EX}, 0}=V_{\mathrm{DD}} \frac{C_{\mathrm{Q}}}{C_{\mathrm{Q}}+C_{\mathrm{AMLED}}} .
$$

Measured transient waveforms of $I_{\mathrm{AMLED}}$ at $\mathrm{T}_{\mathrm{ON}}=35 \mathrm{~ns}$, pulse repetion rate $\left(f_{\mathrm{s}}\right)=10 \mathrm{Mbit} / \mathrm{s}$ are shown in Fig. 5.7 for three different $C_{\mathrm{Q}}$ settings at $V_{\mathrm{DD}}=1.8 \mathrm{~V}$. $C_{\mathrm{AMLED}}$ was measured to be about $650 \mathrm{fF}$ at $V_{\mathrm{AMLED}}=15$ $\mathrm{V}$ (close to $\left.V_{\mathrm{BR}}\right)$. For a lower $C_{\mathrm{Q}}, V_{\mathrm{EX}, 0}$ is lower (Eq. (5.6)) and hence the magnitude of the $I_{\mathrm{AMLED}}\left(=V_{\mathrm{EX}, 0} / R_{\mathrm{AMLED}}\right)$ decreases (Fig. 5.3(a)). The charging rate of $C_{\mathrm{Q}}$ is limited by the $R_{\mathrm{AMLED}}$ (estimated as $\sim 1.4 \mathrm{k} \Omega$ from Fig. 5.3(a)). Simulated transient data (using Spectre [120]) were obtained using a lookup table based model of the DC AMLED $I-V$ characteristics (Fig. 5.3(a)) in combination with a junction capacitance model for $C_{A M L E D}$ (see dashed area in Fig. 5.5(a)). 
a)

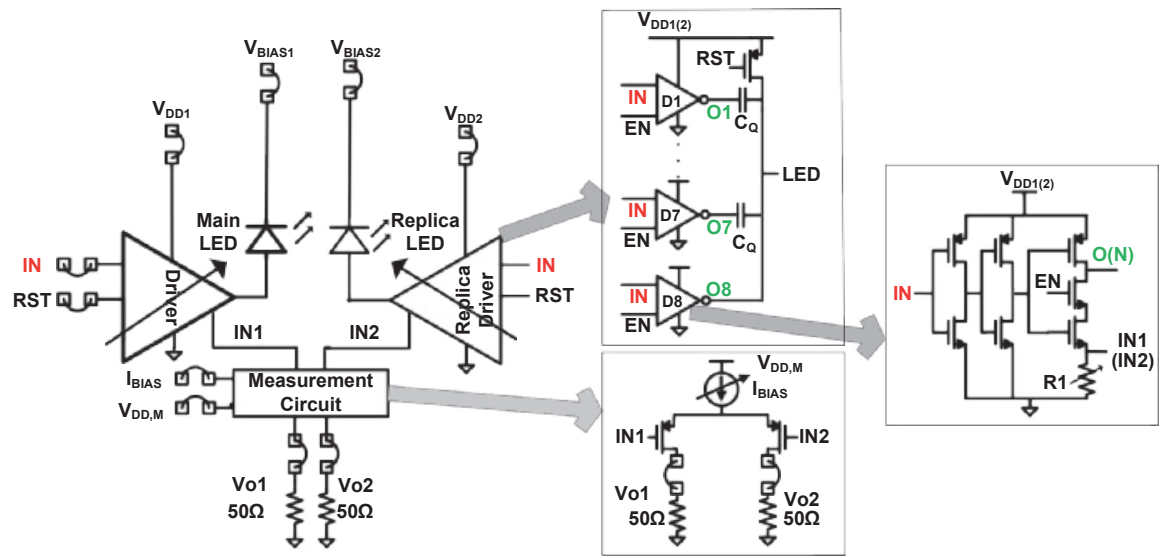

b)

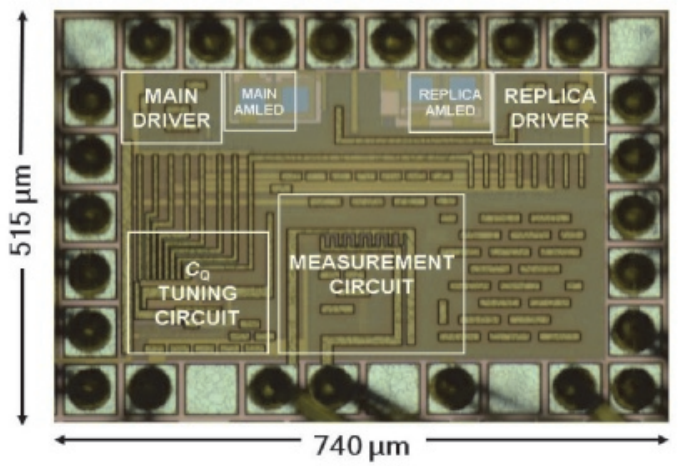

Figure 5.6: (a) Complete schematics of the integrated AMLED driver and the measurement circuit. (b) Micrograph of the implemented chip. The chip was packaged without any top cover to measure vertical light emission from the AMLED. Also, no tiling was used over the AMLED area to avoid light blocking by the backend. Total area of the chip is $0.38 \mathrm{~mm}^{2}$, the area of the main driver is $0.024 \mathrm{~mm}^{2}$ and the area of the AMLED is $0.0033 \mathrm{~mm}^{2}$.

\subsubsection{Energy-per-bit and emitted photon flux per bit of the AMLED}

From an electrical point of view $E_{\mathrm{b}}$ is a key Figure of Merit (FoM) [38]; $E_{\mathrm{b}}$ is defined as the energy required to transfer $Q_{b}$ through the AMLED:

$$
E_{\mathrm{b}}=V_{\mathrm{BIAS}} Q_{\mathrm{b}}
$$

where $V_{\text {BIAS }}$ is the DC bias voltage at the AMLED cathode (Fig. 5.6). $E_{\mathrm{b}}$ is limited by $C_{Q}$ (Eq. (5.5d), Eq. (5.6)):

$$
E_{\mathrm{b}}=V_{\mathrm{BIAS}} Q_{\mathrm{b}} \leqslant V_{\mathrm{BIAS}} C_{\mathrm{Q}} V_{\mathrm{EX}, 0} \leqslant V_{\mathrm{BIAS}} C_{\mathrm{Q}} V_{\mathrm{DD}}
$$

Fig. 5.8(a) shows an example of the measured $E_{\mathrm{b}}$ as a function of $C_{\mathrm{Q}}$. The simulated $E_{\mathrm{b}}$ in Fig. 5.8(a) was obtained using Spectre [120], showing a good agreement with the measured $E_{\mathrm{b}}$. The slight difference could 

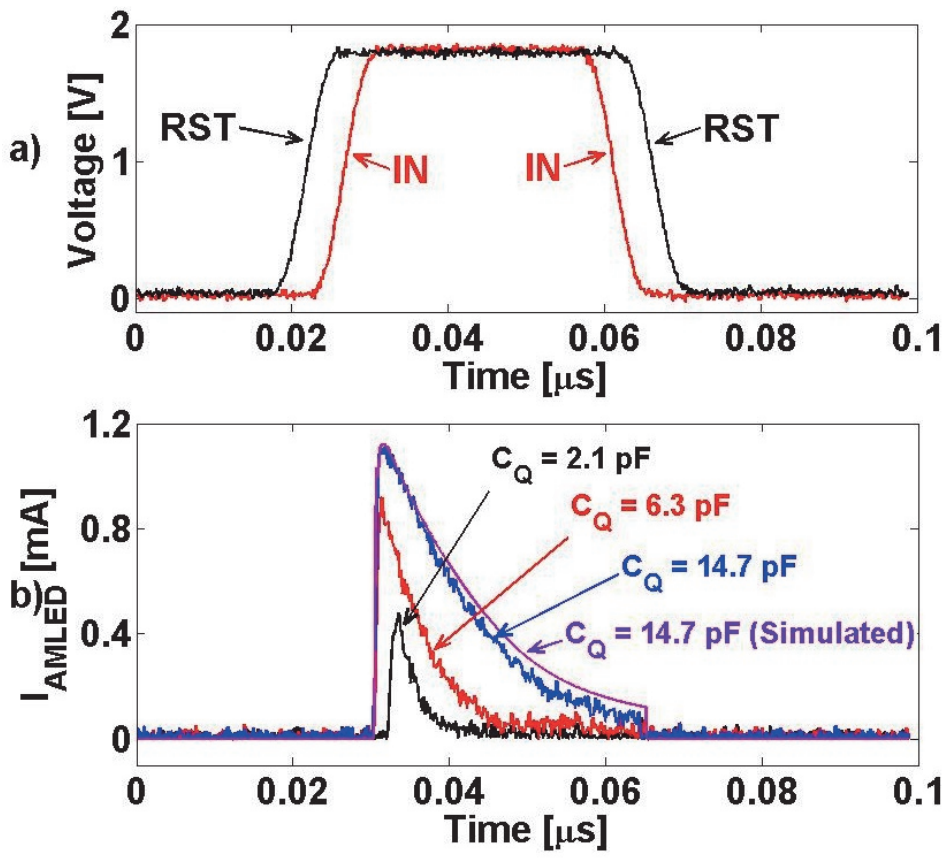

Figure 5.7: (a) Control signals (IN and RST). (b) Measured transient waveforms of $I_{\text {AMLED }}$ for three different $C_{\mathrm{Q}}$ settings at $f_{\mathrm{s}}=10 \mathrm{Mbit} / \mathrm{s}$. For clarity, only three settings are shown. The jitter at lower $C_{Q}$ setting is because of a lower $\mathrm{Q}_{\mathrm{b}}$ (chapter 5). Simulated transient data are also shown only for $C_{\mathrm{Q}}$ $=14.7 \mathrm{pF}$ for clarity.

be explained by the variations in $C_{\mathrm{AMLED}}$ with $V_{\mathrm{AMLED}}$, which has been assumed constant in the simplified model (Fig. 5.5). The loss in the driver circuit was estimated to be about $21 \mathrm{pJ} / \mathrm{bit}$.

For on-chip optical data communication, the lateral photon transmission to a nearby PD is relevant. To measure this, a calibration PD was integrated next to the AMLED (see Figs. 5.2(a)-5.2(b)). The AMLED is operated in OOK mode at $f_{\mathrm{s}}$ and the average PD photocurrent $\left(I_{\mathrm{PD}}\right)$ is measured. From $I_{\mathrm{PD}}$ and $f_{\mathrm{s}}$, the number of photons received at the PD per bit $\left(N_{\text {photons,PD }}\right)$ can be estimated:

$$
N_{\text {photons }, \mathrm{PD}}=\frac{I_{\mathrm{PD}}}{\eta_{\mathrm{PD}} \cdot q_{\mathrm{e}} \cdot f_{\mathrm{s}}},
$$

where $q_{\mathrm{e}}\left(=1.6 \times 10^{-19} \mathrm{C}\right)$ is the elementary charge. The quantum efficiency of the PD ( $\left.\eta_{\mathrm{PD}}\right)$ is assumed to be unity in the wavelength region of interest because the dimensions of the PD are much larger than the absorption length of the photons [12]. Fig. 5.8(b) shows an example of the measured $N_{\text {photons,PD }}$ (on left y-axis) at different $E_{\mathrm{b}}$ settings at $T_{\mathrm{ON}}=35 \mathrm{~ns}$ and $f_{\mathrm{s}}=10$ Mbit/s. 

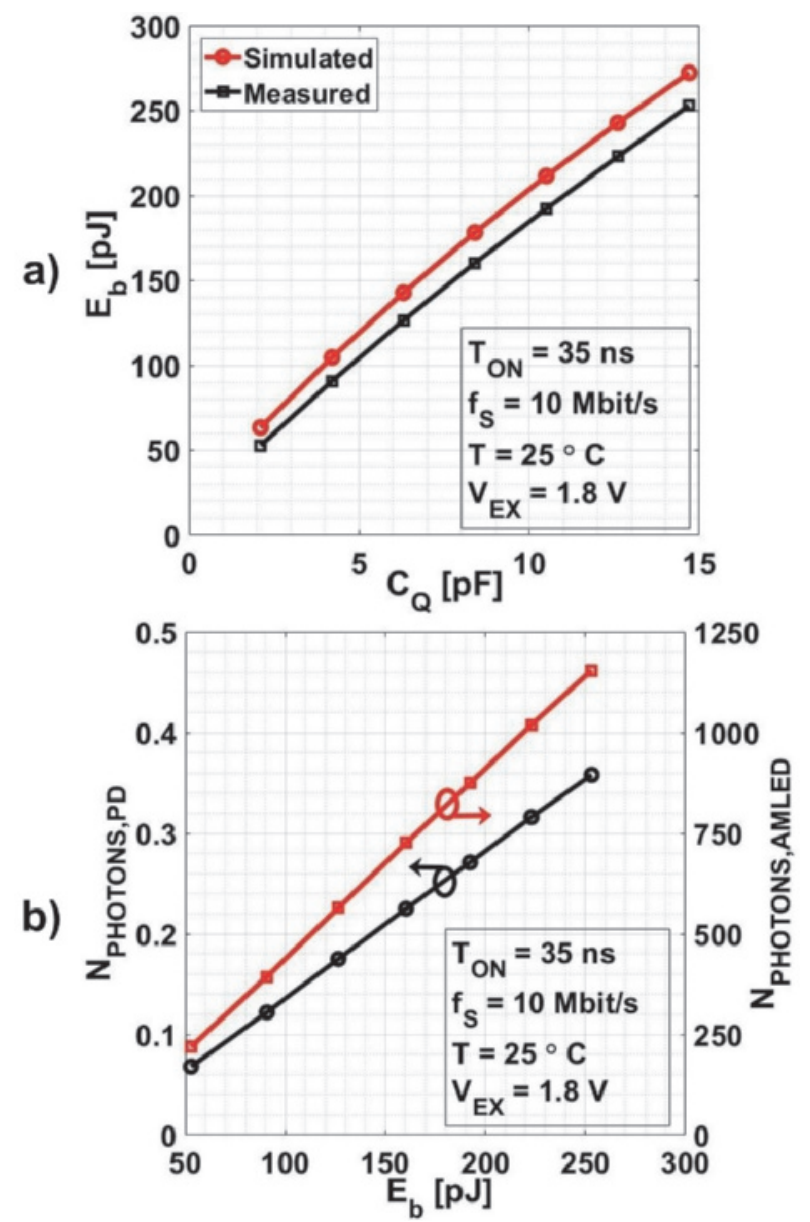

Figure 5.8: (a) Measured and simulated $E_{\mathrm{b}}$ as a function of $C_{\mathrm{Q}}$. (b) Estimated $N_{\text {photons,PD }}$ and $N_{\text {photons,AMLED }}$ as a function of $E_{\mathrm{b}} . N_{\text {photons,PD }}$ and $N_{\text {photons,AMLED }}$ are photons per bit.

From $\eta_{\mathrm{TE}}=3 \times 10^{-4}$ (as derived in section 5.3 for this sub-optimum

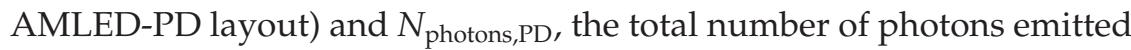
by the AMLED per bit ( $\left.N_{\text {photons,AMLED }}\right)$ for different $E_{\mathrm{b}}$ settings can be estimated (Eq. (5.10)):

$$
N_{\text {photons,AMLED }}=\frac{N_{\text {photons,PD }}}{\eta_{\mathrm{TE}}} .
$$

An example of the estimated $N_{\text {photons,AMLED }}$ is also shown in Fig. 5.8(b) (on right y-axis). In turn, from $N_{\text {photons,AMLED }}$ and the number of electrons through the AMLED per bit ( $\left.N_{\text {electrons,AMLED }}=\frac{Q_{\mathrm{b}}}{q_{\mathrm{e}}}=\frac{E_{\mathrm{b}}}{V_{\text {BIAS }} q_{\mathrm{e}}}\right)$, the internal quantum efficiency (IQE) of the AMLED (Eq. 5.1) is estimated to be about $1.4 \times 10^{-5}$, comparable to what was also reported for Si earlier [16]. Using $I Q E$ and $\eta_{\mathrm{TE}}$ (section 5.3), $\eta_{\text {system }}$ is estimated as $4 \times 10^{-9}$ [13]. 

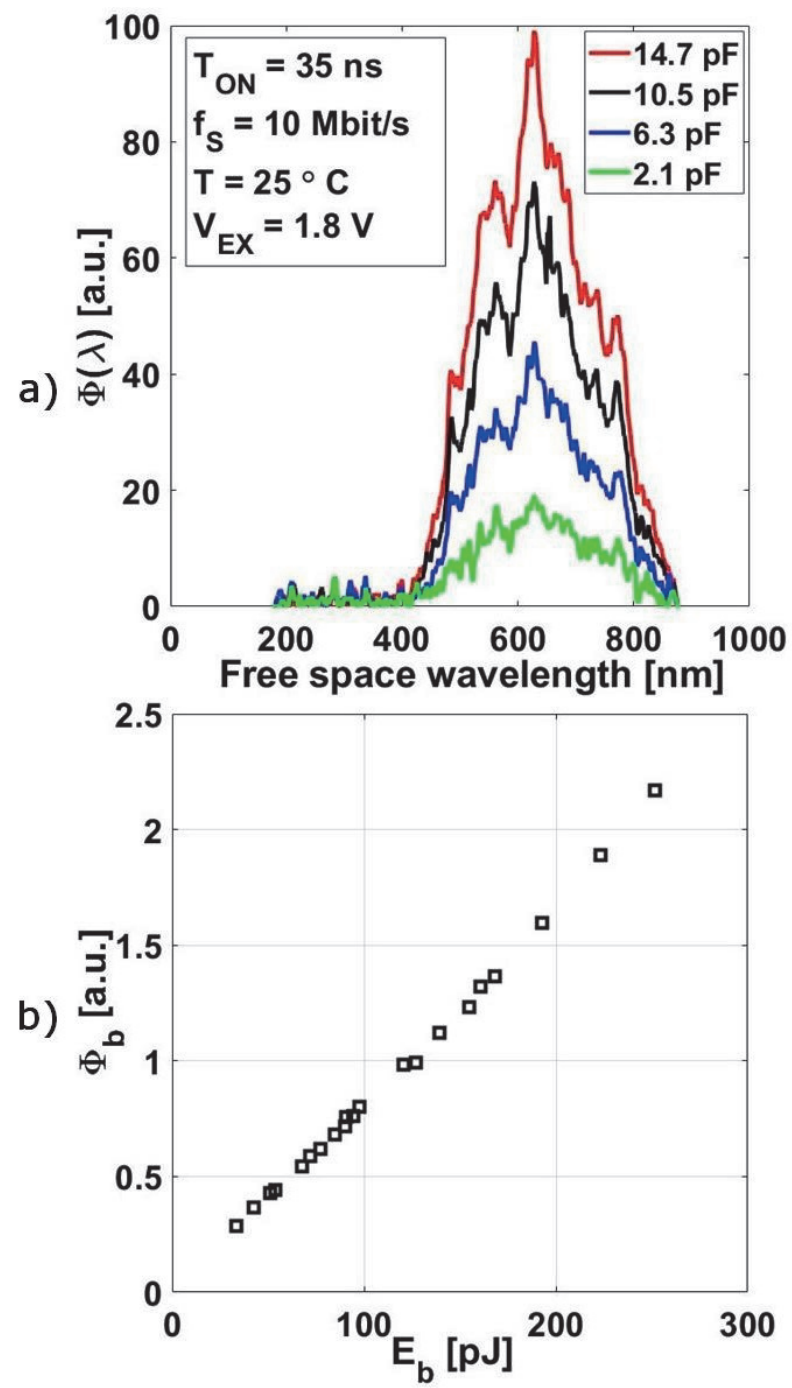

Figure 5.9: (a) Measured vertical photon spectral flux density for several $C_{Q}$ using an Avantes ADC-1000-USB spectrometer with an integration time of $30 \mathrm{~s}$. (b) Measured vertical photon flux density per bit $\left(\Phi_{\mathrm{b}}\right)$ as a function of $E_{\mathrm{b}}$.

Fig. 5.9(a) shows the measured $\Phi(\lambda)$ of the vertical emission of the AMLED (along the z-axis in Fig. 5.4), driven at $T_{\mathrm{ON}}=35 \mathrm{~ns}, f_{\mathrm{s}}=10$ $\mathrm{Mbit} / \mathrm{s}$, for several values of $C_{\mathrm{Q}}$, demonstrating that the $\Phi(\lambda)$ is almost proportional to $C_{Q}$, with a minor effect on the spectrum. Only four $C_{Q}$ settings are shown for clarity. The increase in intensity with increasing $C_{Q}$ is because of increasing $Q_{\mathrm{b}}$.

Fig. 5.9(b) shows the vertical photon flux density per bit, $\Phi_{b}=\frac{1}{f_{s}} \int_{\lambda} \Phi(\lambda) d \lambda$, as a function of $E_{\mathrm{b}}$, demonstrating a linear relation between $E_{\mathrm{b}}$ and $\Phi_{\mathrm{b}}$. 

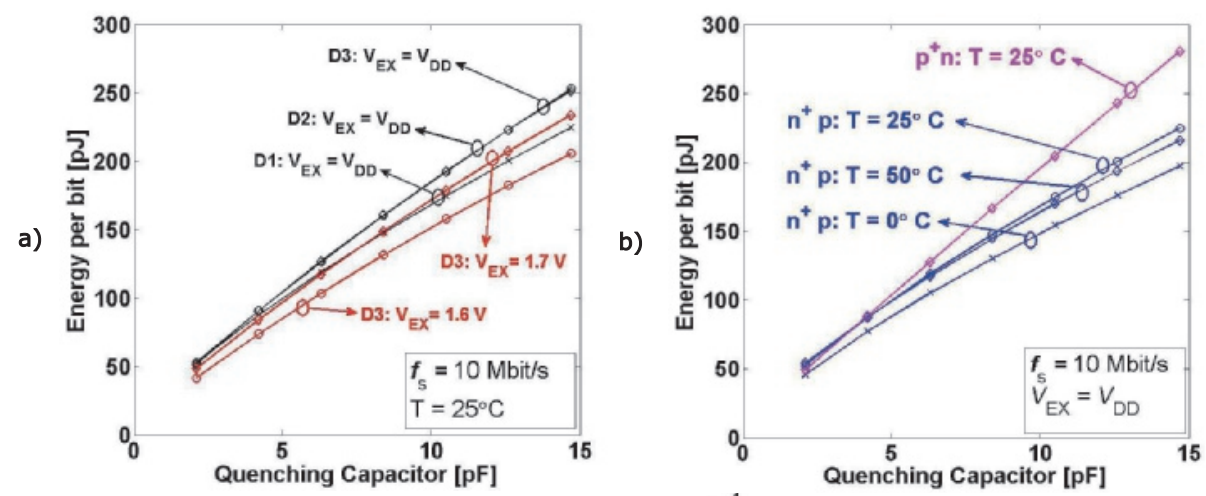

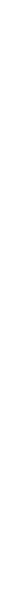

Figure 5.10: Measured $E_{\mathrm{b}}$ of an AMLED driven by the self-quenched driver circuit compared to using a traditional non-quenched driver circuit. (a) The $\mathrm{n}^{+} \mathrm{p}$ AMLED: process and voltage variations. (b) The $\mathrm{n}^{+} \mathrm{p}$ AMLED: temperature variations and $E_{\mathrm{b}}$ vs $C_{\mathrm{Q}}$ for the $\mathrm{p}^{+} \mathrm{n}$ AMLED. (c) $E_{\mathrm{b}}$ for nonquenched circuits for the $\mathrm{n}^{+} \mathrm{p}$ and $\mathrm{p}^{+} \mathrm{n}$ AMLED. (d) Effect of pulse width $\left(T_{\mathrm{ON}}\right)$ on $E_{\mathrm{b}}$ for the $\mathrm{n}^{+} \mathrm{p}$ AMLED.

\subsubsection{Robustness of $E_{\mathrm{b}}$}

The robustness of this driver circuit to process, voltage, temperature, design and pulse width $\left(T_{\mathrm{ON}}\right)$ variations is demonstrated using $E_{\mathrm{b}}$ as the FoM. Note that using the results shown in Fig. 5.8(b), $E_{\mathrm{b}}$ can be translated into $N_{\text {photons,PD }}$.

For process variations, three different samples of the $\mathrm{n}^{+} \mathrm{p}$ AMLED, D1, D2 and D3 from the same processing batch were used.

To explicitly show robustness of the proposed driver circuit with design variations, $E_{\mathrm{b}}$ for a $\mathrm{p}^{+} \mathrm{n}$ diode was also measured, driven by a replica driver circuit (Fig. 5.6). The physical properties of both the square shaped AMLEDs are summarized in the table below: 


\begin{tabular}{|l|l|l|l|l|l|}
\hline & shape & Edge length & $V_{\text {BR }}$ & $R_{\text {AMLED }}$ & $C_{\text {AMLED }}$ \\
\hline $\mathrm{n}^{+} \mathrm{p}$ & square & $45 \mu \mathrm{m}$ & $\sim 17 \mathrm{~V}$ & $1.4 \mathrm{k} \Omega$ & $650 \mathrm{fF}$ \\
\hline $\mathrm{p}^{+} \mathrm{n}$ & square & $45 \mu \mathrm{m}$ & $\sim 14.4 \mathrm{~V}$ & $560 \Omega$ & $585 \mathrm{fF}$ \\
\hline
\end{tabular}

Figs. 5.10(a)-5.10(b) show measured $E_{\mathrm{b}}$ as a function of $C_{\mathrm{Q}}$ across PVT and AMLED design variations, demonstrating that $E_{\mathrm{b}}$ is hardly affected by these variations but can properly be tuned by setting different $C_{Q}$ values.

$E_{\mathrm{b}}$ is non-linear with respect to $C_{\mathrm{Q}}$ because the pulse width $\left(T_{\mathrm{ON}}\right)$ is not sufficient to charge large $C_{Q}$ completely (Eq. (5.5d) and Fig. 5.5). A slight difference between the $E_{\mathrm{b}}$ of the $\mathrm{n}^{+} \mathrm{p}$ AMLED and the $\mathrm{p}^{+} \mathrm{n}$ AMLED is because of the different $R_{\mathrm{AMLED}}, C_{\mathrm{AMLED}}$ and $V_{\mathrm{BR}}$. The higher linearity in $E_{\mathrm{b}}$ with respect to $C_{\mathrm{Q}}$ in this $\mathrm{p}^{+} \mathrm{n}$ AMLED is because of its low $R_{\mathrm{AMLED}}$.

Fig. 5.10(c) shows $E_{\mathrm{b}}$ for conventional (non-quenched) OOK drivers that show a large sensitivity to PVT variations and (especially) design variations. The measured $E_{\mathrm{b}}$ for the two types of AMLEDs are drastically different which is because of the different $I-V$ characteristics.

In data communication using OOK, the pulse width ( $T_{\mathrm{ON}}$ in Fig. 5.5) is of importance. Fig. 5.10(d) shows the impact of the pulse width $\left(T_{\mathrm{ON}}\right)$ on $E_{\mathrm{b}}$ : for the self-quenched driver, the $E_{\mathrm{b}}$ is mainly determined by the selected $C_{\mathrm{Q}}$, for pulse repetition rate $\left(f_{\mathrm{s}}\right)$ to $\sim 10 \mathrm{Mbit} / \mathrm{s}$. At lower $T_{\mathrm{ON}}$, the $E_{\mathrm{b}}$ drops due to mainly $R_{\mathrm{AMLED}}$ that determines the maximum $Q_{\mathrm{b}}$ and hence the $E_{\mathrm{b}}$ (Eq. (5.5d), Eq. (5.8)).

For comparison, the non-quenched operation of the AMLED in Fig. $5.10(\mathrm{~d})$ shows a strong $T_{\mathrm{ON}}$ (hence timing) dependency. The simulated $E_{\mathrm{b}}$ (using Spectre) shows a good agreement with the measured $E_{\mathrm{b}}$. For the nonquenched driver circuit, $E_{\mathrm{b}}$ can be estimated as $E_{\mathrm{b}} \approx V_{\mathrm{BIAS}} I_{\mathrm{AMLED}} T_{\mathrm{ON}}=$ $V_{\mathrm{BIAS}}\left(V_{\mathrm{EX}} / R_{\mathrm{AMLED}}\right) T_{\mathrm{ON}}$. Using the self-quenched circuit, $E_{\mathrm{b}}$ (thus power dissipation) is always lower than $\mathrm{C}_{\mathrm{Q}} V_{\mathrm{DD}} V_{\mathrm{BIAS}}$ independent of $\mathrm{T}_{\mathrm{ON}}$ (Eq. (5.8)).

\subsection{Application in opto-couplers}

In this section, first the potential of the proposed optical transmitter for application in opto-couplers is discussed. In a further section, the aspects of the bit rate are addressed.

\subsubsection{Optocouplers in CMOS technology}

Although this work mainly focused on the transmitter in a fully Si-integrated optical link, the demonstrated optical transmitter aims at applications in a complete optical link in Si. For the minimum $E_{\mathrm{b}}$ setting $(\sim 53 \mathrm{pJ} /$ bit $)$, the $\mathrm{n}^{+} \mathrm{p}$ AMLED emits 220 photons per bit isotropically (Fig. 5.8(b)). Using Fig. 5.8 and the model presented in section 5.3, it is possible to optimize the optical link geometry to receive e.g. 5 photons at the PD out of $N_{\text {photons,AMLED }}$. To receive more than 5 photons out of 220 , it is required that $\eta_{\mathrm{TE}}>2 \times 10^{-2}$. For instance, when the AMLED and the PD are aligned as shown in Fig. 


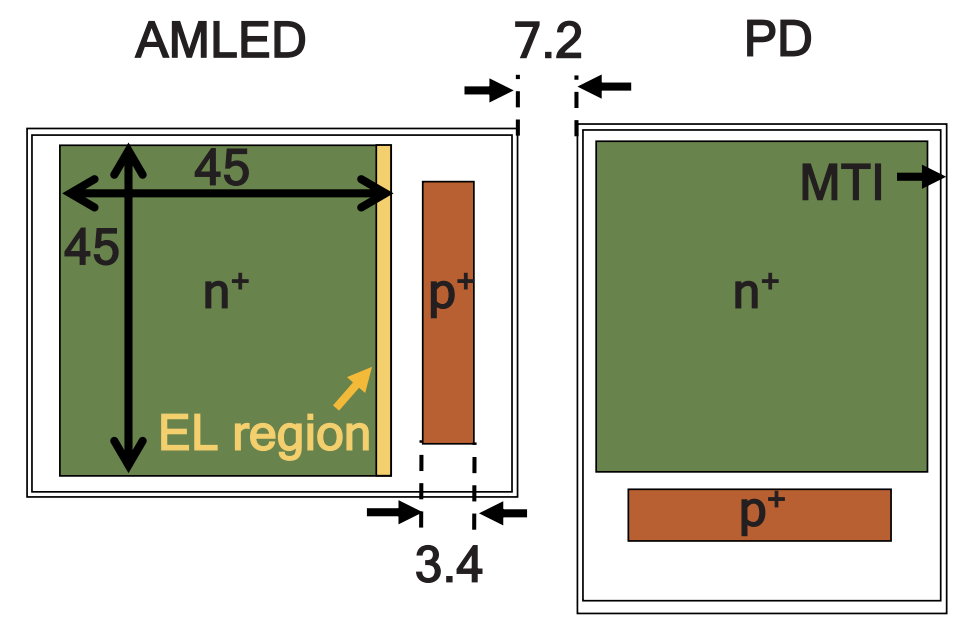

Figure 5.11: Proposed layout of the AMLED and the PD to increase the $\eta_{\mathrm{TE}}$.

5.11 , the $\eta_{\mathrm{TE}}$ of the link is estimated to be $2 \times 10^{-2}$ using the method in section 5.3. Further suggestions to improve $\eta_{\mathrm{TE}}$ such as patterning the link, improving the AMLED design have been extensively discussed in [13]. Obviously, it is desired to have an optimized optical transmission path for waveguiding the photons from the AMLED to the PD. This will improve the coupling quantum efficiency, as well as reduce the crosstalk among multiple channels in a multi-channel communication environment.

If 5 photons are received at the PD per bit, this is typically sufficient to operate a well-designed Single Photon Avalanche Diode (SPAD) as a PD [22, 23, 109, 121-124]. SPADs are p-n junctions biased above $V_{\text {BR }}$ so that an incoming photon in the depletion region can generate free carriers thus triggering an avalanche. The macroscopic avalanche current can then be easily measured by using digital simple read out circuitry. The design of SPADs and SPAD read out circuits are well-known in CMOS technologies [23, 122, 123]. Recently, a SPAD designed in the same technology as presented in this work has been reported [79].

Further, in the current link geometry a large jitter would occur if 5 photons per bit were statistically received at the PD. This is because many free carriers are generated by the photons outside the depletion region which subsequently diffuse towards the depletion region triggering the SPAD after some ill determined diffusion time. Therefore, it is recommended to design links with a higher $\eta_{\mathrm{TE}}$ and to capture photons mainly in the depletion region. This could be achieved by using e.g., less doping in the PD which results in a wider depletion region [12]. 


\subsubsection{Bit rate}

The bit rate in such an optical link would ultimately be limited by the speed of the detector (SPADs or the PDs). AMLEDs can be designed to achieve a relatively high speed (see chapter 4).

For SPADs, the achievable bit rate is limited by their deadtime requirement. To reduce the unpredictable and hence undesired aferpulsing phenomena in SPADs, the deadtime of the SPADs after each photon counting event has to be increased, which limits the bit rate of the SPADs [23, 79]. For the SPADs that were reported in this technology [79], the bit rate would be limited to about $10 \mathrm{Mbit} / \mathrm{s}$ based on their reported deadtime ( $100 \mathrm{~ns})$.

Using conventional photodiodes, higher bit rates can be achieved, however at the cost of increased power consumption in the AMLED. This is because of its required continuous operation mode to increase the signalto-noise ratio (thereby to reduce the bit error rate) at the PD. A data rate of $3 \mathrm{Gbit} / \mathrm{s}$ has been reported with an integrated Si receiver in a standard 180 nm CMOS technology using off chip illumination [125].

\subsection{Conclusion}

In this chapter, a low power monolithically integrated optical transmitter using avalanche mode LEDs (AMLEDs) was designed in a standard 140 nm SOI CMOS technology. The novel self-quenched driver circuit resolves many physics issues related to power dissipation and PVT variations of an AMLED. This work successfully demonstrates a low power wide spectrum optical transmitter in a CMOS technology which can be integrated with standard silicon photodetectors. It further reinforces the promise of enabling AMLEDs as light sources in silicon CMOS technologies for monolithic integration of optocouplers. [13, 16, 41, 116]. 


\title{
CHAPTER
}

\section{OPTOCOUPLING IN CMOS}

\begin{abstract}
For on-chip data communication with galvanic isolation, a monolithically integrated optocoupler is strongly desired. For this purpose, silicon (Si) avalanche mode LEDs (AMLEDs) offer a great potential. However such AMLEDs have a relatively low internal quantum efficiency (IQE) and high power consumption. For the first time, in this work, data communication in a monolithically integrated optocoupler is experimentally demonstrated. The novelty of this work is the use of highly sensitive single-photon avalanche diodes (SPADs) for photodetection to compensate for the low IQE of AMLEDs. We investigated our optocoupler realized in a standard $140 \mathrm{~nm}$ CMOS SOI technology, without post-processing, for various LED designs and points of operation. The power consumption of the AMLEDs is minimized through a novel AMLED design and employment of a low power LED driver circuit. The advantages of AMLEDs over forward biased Si LEDs are also demonstrated. For the best AMLED design, the achievable data rate is few Mbps and the energy consumption a few $\mathrm{nJ} / \mathrm{bit}$. The active area of the proposed systems is $<0.01 \mathrm{~mm}^{2}$.
\end{abstract}




\subsection{Introduction}

Many smart power applications require data communication between different voltage domains with galvanic isolation. Currently this is achieved using inductive isolators, capacitive isolators or discrete optocouplers [20]. Such isolators have various disadvantages: they are large in size, source of extra complexity and costly. In addition, some are also sensitive to external interferences. Conversely, when monolithically integrated, optocouplers in standard CMOS technologies are largely free of such issues. Communication by means of light is also attractive because light is robust to external electrical or magnetic interferences.

So far, optocouplers have not been monolithically integrated in standard CMOS because of the poor overlap between the emission spectrum of a (traditional) forward biased Si LED and the responsivity of a Si photodetector (PD) (Fig. 6.1(a)). Therefore, in most discrete optocouplers, LEDs are designed using III-V compounds e.g. GaAs [11] and PDs are typically implemented using Si. This heterogeneous integration makes fabrication more complicated and thereby expensive.

In avalanche mode, Si AMLEDs have an emission spectrum in the visible wavelength region [14]; this spectrum has a significant overlap with the responsivity of Si PDs (Fig. Fig. 6.1(b)). The spectrum of AMLEDs is due to the radiative recombination of high-energy carriers during avalanche breakdown [14]. However, AMLEDs have a poor IQE $\left(^{-5}\right)[15,71]$. Also, systems implemented with AMLEDs can be power hungry because of the avalanche operation under high voltage [64,71].

The significant development of SPADs in CMOS [123] opens new routes for monolithic integration of optocouplers. SPADs are highly sensitive PDs and can be used to compensate for the poor IQE of AMLEDs. SPADs also have a count rate of up to tens of Mbps, which is sufficient for many smart power applications. For the first time, we report the integration of Si AMLEDs-SPADs for optocoupling applications (Fig. 6.2). It was shown that the breakdown voltage $\left(V_{\mathrm{BR}}\right)$ of AMLEDs can be reduced down to $6 \mathrm{~V}$ without degrading their IQE [67]. In this work, we have designed novel AMLED structures to reduce their $V_{\mathrm{BR}}$ for low power consumption and then we employed these AMLEDs for optocoupling focusing on power consumption, speed and bit-error-rate (BER). The LEDs and SPADs were designed in a $140 \mathrm{~nm}$ CMOS SOI technology; the LED driver and SPAD quenching-and-recharge circuits (QRCs) were implemented externally using off-the-shelf components. The proposed optocoupler (LEDs and SPADs) occupy much less area $\left(\sim 0.008 \mathrm{~mm}^{2}\right)$ compared to inductors $\left(\sim 1.9 \mathrm{~mm}^{2}\right.$ in [126]) and capacitors $\left(\sim 1.6 \mathrm{~mm}^{2}\right.$ in [39]) of inductive and capacitive isolators respectively. The LED driver and QRCs can be integrated on-chip at relatively low area requirements $[71,123]$. The results suggest a great potential of the AMLED-SPAD combination for monolithic optocoupling applications. 

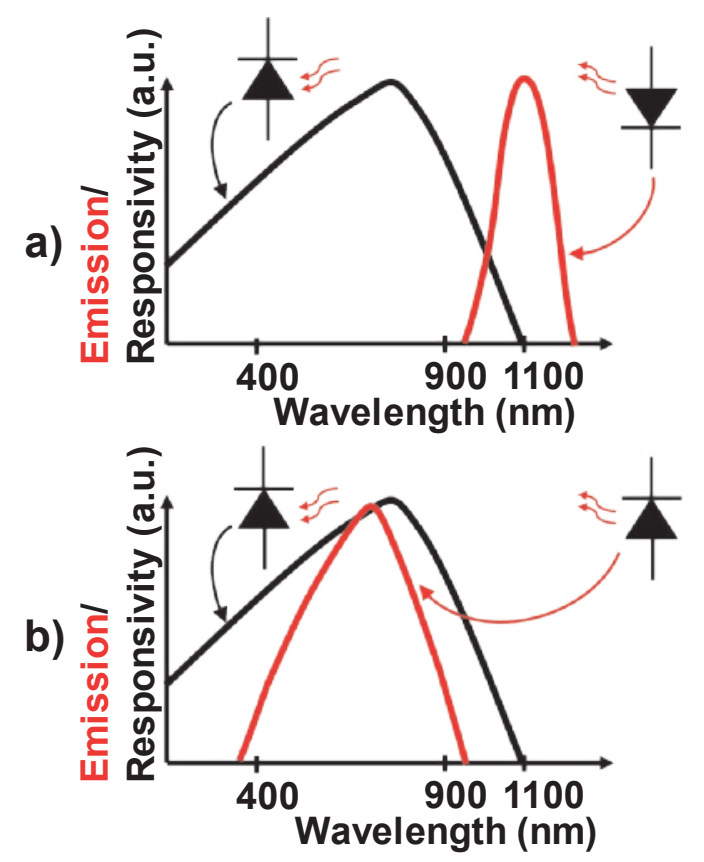

\subsubsection{AMLEDs and SPAD designs}

Fig. 6.3 shows schematic cross-sections of the two LEDs (A1,A2) and the SPAD (S1). A1 is a vertical $\mathrm{n}^{+}$-pwell diode. For optimized light emission with a minimal $V_{\mathrm{BR}}$, A2 was implemented using closely spaced $\mathrm{p}^{+}-\mathrm{n}^{+}$

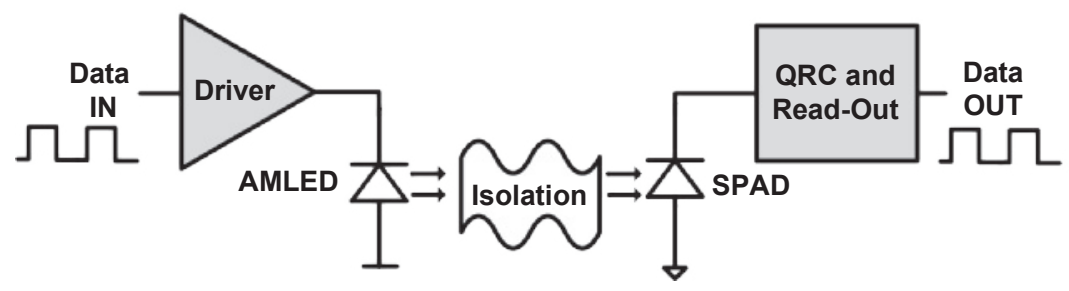

Figure 6.2: Proposed optical link comprising of AMLEDs and SPADs. The LED driver circuit drives the LED in response to the incoming data to transmit photons across the isolation barrier. Some of these transmitted photons are detected across the barrier by the SPADs with quenching-andrecharge circuits (QRCs). The data is demodulated from the output of QRCs. 

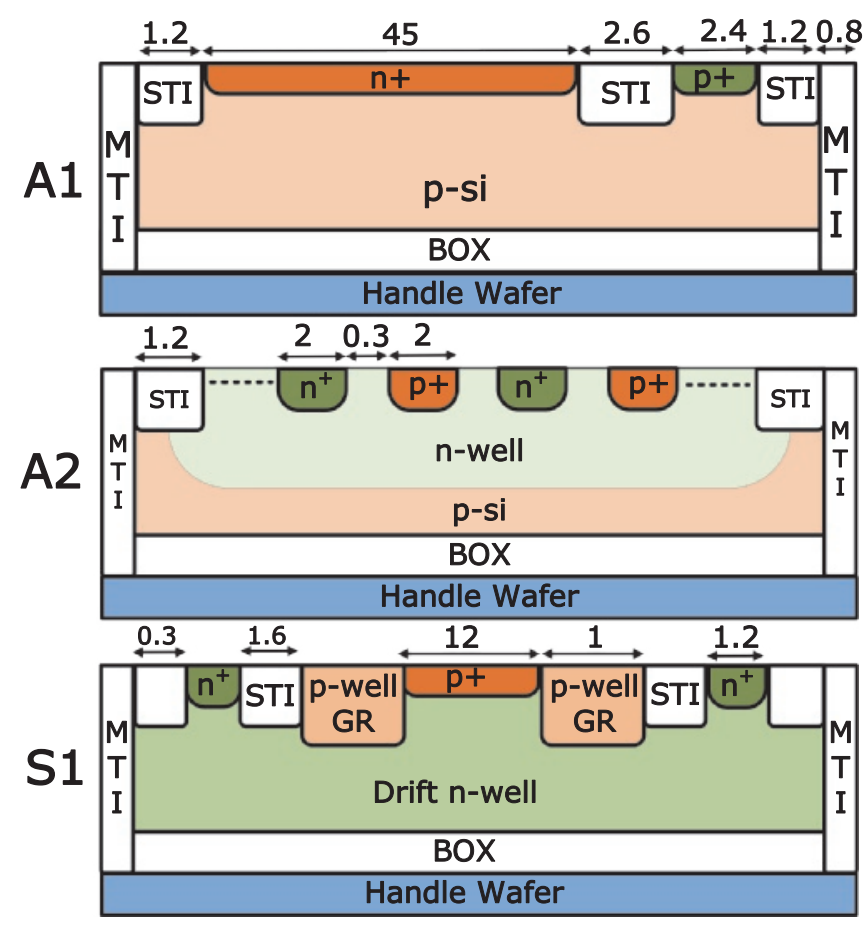

Figure 6.3: Schematic cross-section (not to scale) of LEDs (A1, A2) and SPAD (S1); all dimensions in $\mu \mathrm{m}$. Medium Trench Isolation (MTI) layers provide galvanic isolation between LEDs/SPADs.

layers in an n-well. To reduce the dark count rate (DCR) of S1, the depletion region was isolated from the shallow trench isolation using a lowly doped guard ring [66]. The galvanic isolation was implemented using medium trench isolation (MTI); the MTI in this technology can provide an isolation of $>100 \mathrm{~V}$ [36]. Fig. 6.4 shows the micrograph of the devices A1-S1 and A2-S1. A "C"-shaped layout of A2 was designed to increase the coupling efficiency between $\mathrm{A} 2$ and $\mathrm{S} 1$.

Fig. 6.5 shows the reverse $I-V$ characteristics of the devices. The high leakage current for A2 is most likely because of the presence of band-toband tunneling. This was verified by the temperature dependence of the $V_{\mathrm{BR}}$. For A2, $V_{\mathrm{BR}}$ was practically constant between $0-75^{\circ} \mathrm{C}$ : possibly because the negative temperature dependency of Zener breakdown counteracts the positive temperature dependency of avalanche breakdown [12]. Fig. 6.6 shows the AMLED light emission profiles and spectra.

The DCR of the SPADs were measured using a passive QRC. Fig. 6.7 shows the dependence of the DCR on the excess bias voltage $V_{\mathrm{EX}}$ (the excess voltage at which the SPAD is biased above breakdown) for different temperatures. The inset also shows the DCR measurement setup schematics. Fig. 6.8 shows the photon detection probability (PDP) of these SPADs, indicating their higher sensitivity in the visible wavelengths. 


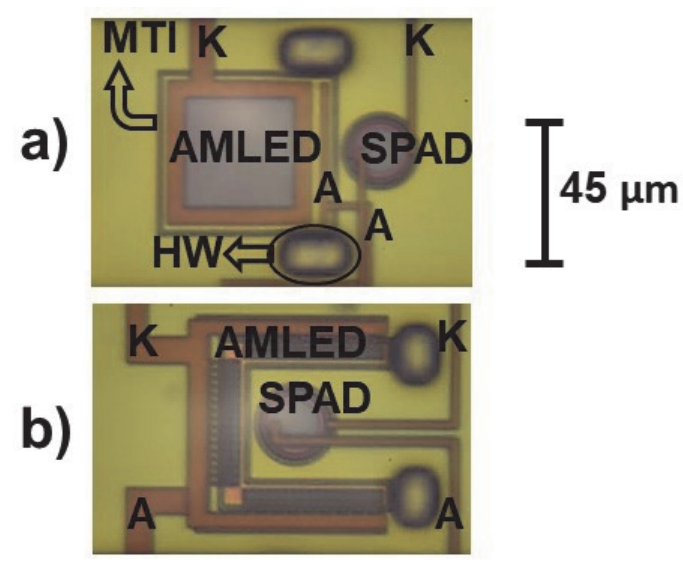

Figure 6.4: Micrograph of (a) A1-S1 (b) A2-S1. "K" and "A" indicate cathode and anode respectively. Closest distance between the active areas of the LED and the SPAD in both cases is $10 \mu \mathrm{m}$. The " $\mathrm{C}$ " shaped layout of A2 was chosen to increase coupling efficiency $\left(\eta_{\mathrm{CE}}\right)$ between LED and PD (Fig. 6.10). A circular SPAD is used to reduce edge breakdown. "HW" is the handle wafer contact, acting as heat sink.

S1 was biased above breakdown and the avalanche firing rate (AFR) of S1 was measured when the corresponding LED was biased at the same DC current level in avalanche mode (AM) or in forward mode (FM), i.e. an FMLED. In dark conditions, AFR is equivalent to DCR. For the AM operation, the measured AFR was $\sim 4 \mathrm{x}$ higher than that for the FM operation (Fig. 6.9). Despite that the IQE for an FMLED is about two orders of magnitude higher [127] than that of an AMLED, Fig. 6.9 demonstrates better spectral overlap for AMLED-SPAD than that for FMLED-SPAD. Fig. 6.10 shows the DC coupling between the A1-S1 and A2-S1 when S1 was operated as a "conventional" photodiode. A higher coupling efficiency ( $\left.\eta_{C E}\right)$ is observed for A2-S1 than for A1-S1.

\subsubsection{LED driver circuit schematics}

Fig. 6.11 describes the equivalent circuit of our LED driver. It is a selfquenched driver circuit that limits the amount of charge-per-bit $\left(Q_{b}\right)$ and energy-per-bit $\left(E_{\mathrm{b}}\right)$ through the LED. For more details about this driver circuit, please refer to chapter 5 in which a fully integrated version was presented.

\subsubsection{Data modulation and BER definition}

For SPADs, the data are encoded in their timing response [109]. Therefore, an appropriate modulation scheme for data communication using SPADs is a pulse position modulation (PPM) [109]. Fig. 6.12 shows the schematics of the LED driver control signals IN and RST for this modulation scheme. 


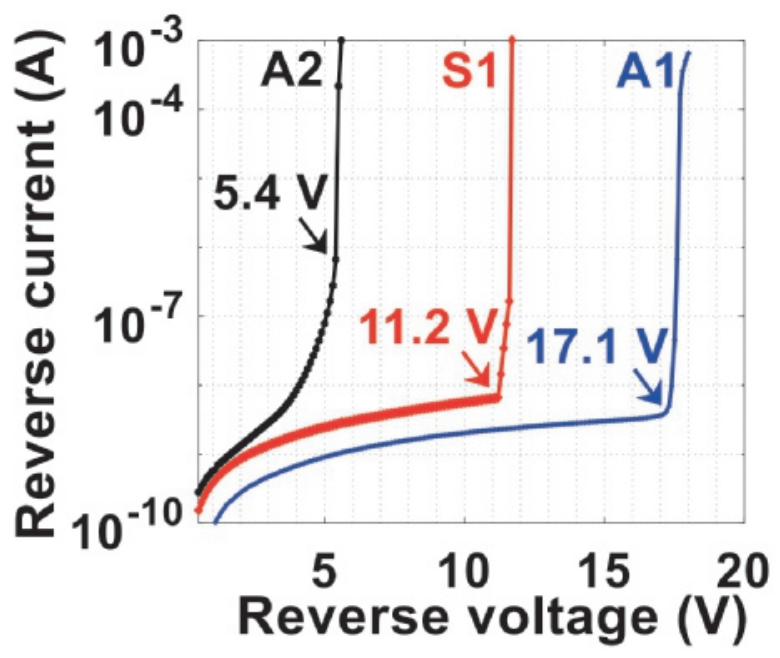

Figure 6.5: Reverse $I-V$ characteristics of the devices measured using an Agilent B2901A with $1 \mathrm{~s}$ integration time. $V_{\mathrm{BR}}$ (indicated) is defined as the voltage at which $I_{\mathrm{R}}$ starts to sharply increase. $V_{\mathrm{BR}}$ for A1 had a temperature coefficient of $\sim 16 \mathrm{mV} / \mathrm{K}$ whereas $V_{\mathrm{BR}}$ for $\mathrm{A} 2$ showed negligible temperature dependency. The temperature coefficient of $V_{\mathrm{BR}}$ for $\mathrm{S} 1$ was measured to be $\sim 8 \mathrm{mV} / \mathrm{K}$.
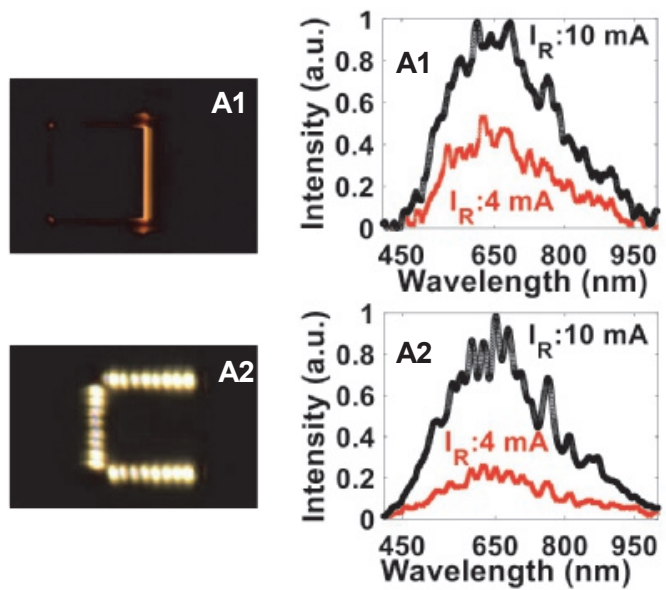

Figure 6.6: (Left) Visible light emission profiles from A1 and A2 (on same scale as Fig. 6.4). AMLEDs were biased at a constant reverse DC current of $10 \mathrm{~mA}$ and the images were captured using a Nikon D3100 camera using $30 \mathrm{~s}$ integration time. For A1, light is emitted mostly from the edge closest to the $\mathrm{p}^{+}$region due to high field and current crowding [71]. For A2, light is emitted from the regions between the $\mathrm{n}^{+}$and $\mathrm{p}^{+}$regions, indicating a lateral breakdown. (Right) Normalized emission spectra of the two AMLEDs for two $I_{R}$ values. 


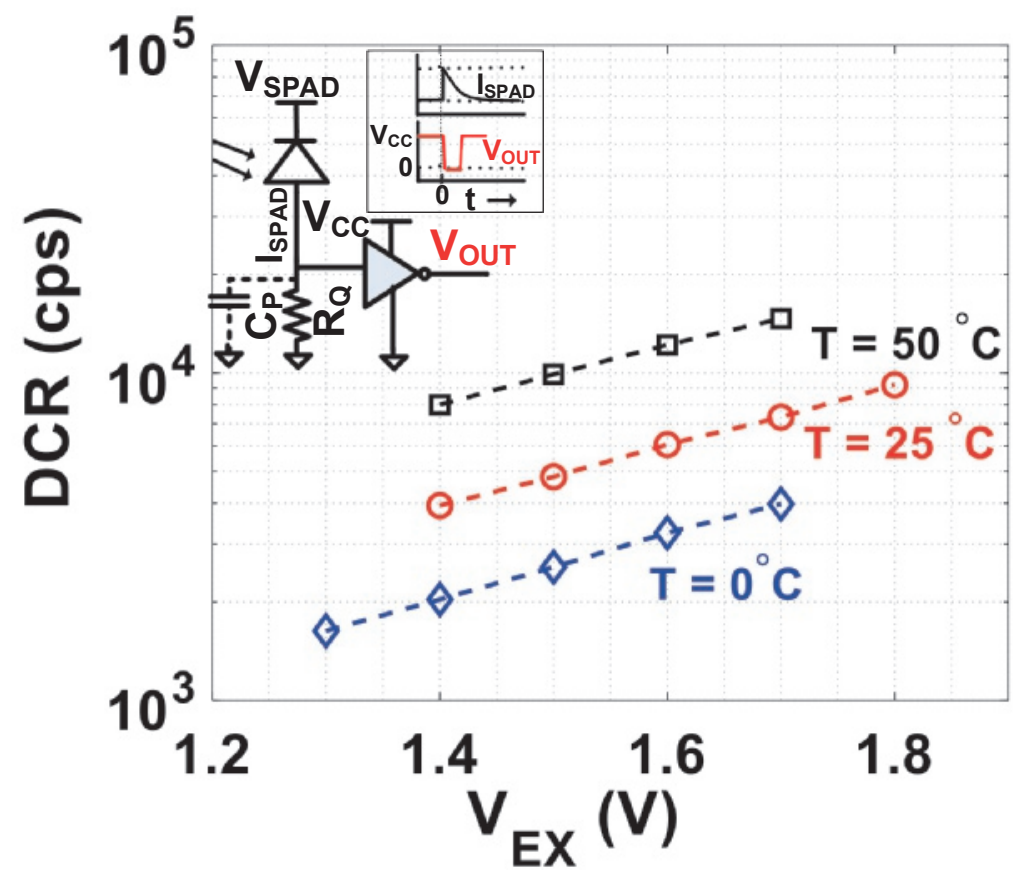

Figure 6.7: Measured DCR for S1 (12 $\mu \mathrm{m}$ diameter) as a function of $V_{\mathrm{EX}}=$ $\left(V_{\mathrm{SPAD}}-V_{\mathrm{BR}}\right)$ for different temperatures. At lower temperatures, DCR is reduced due to reduced thermal generation of dark carriers. Inset depicts the schematic of the measurement setup and illustrative transient waveforms. A passive $\mathrm{QRC}$ is used with a quenching resistance $\left(R_{\mathrm{Q}}\right)$ of $20 \mathrm{k} \Omega$ and $V_{\mathrm{CC}}$ $=2 \mathrm{~V}$.

A PRBS data sequence with a length of $2^{10}$ bits was generated and the performance of the optical links was measured in response to this data sequence across all LEDs and operating conditions.

The received data is decoded from the timing response of the SPAD: if the SPAD is triggered only during the first half of the bit duration, data bit is demodulated as a " 0 ", and if the SPAD is triggered only during the last half of the bit duration, data bit is demodulated as " 1 ". For all other cases, data cannot be reliably decoded and those cases were counted as bit-error (Note: BER $>0.5$ is possible as per this definition). Using the demodulated data, the BER of the link was estimated.

\subsection{Optical Link Performance}

Fig. 6.13 shows an example of the measured transients of the control signals of the driver circuit, $I_{\text {AMLED }}$ and output of QRC ( $\left.V_{\text {OUT }}\right)$ at the specified operating conditions. Figs. 6.14 and 6.15 show the estimated BER of the system for different operating conditions when the LEDs were operated in FM and AM respectively. Both in AM and FM, A2-S1 show a lower 


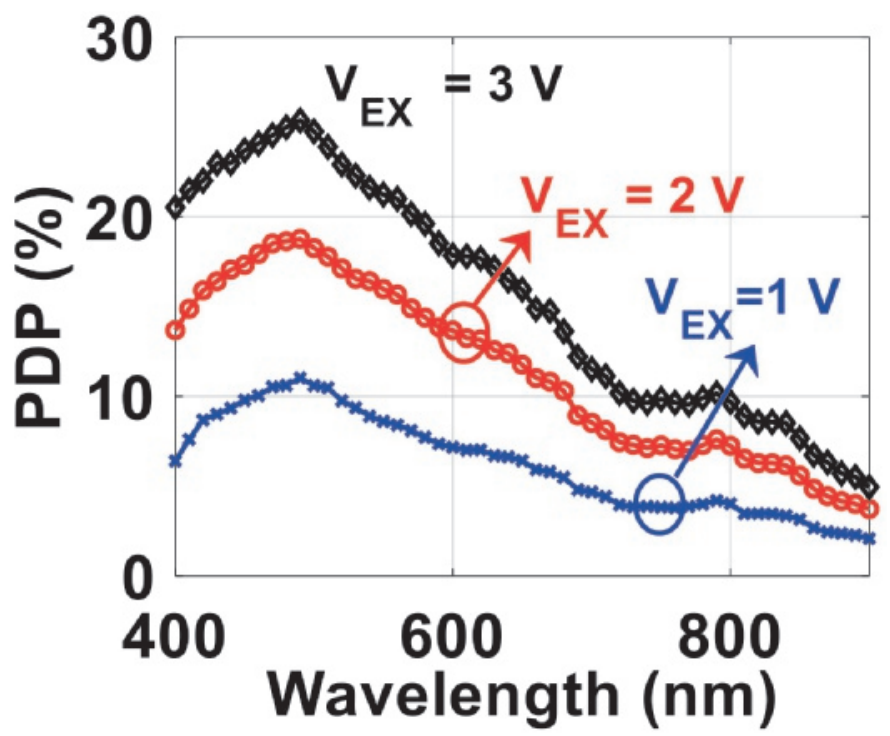

Figure 6.8: Photon detection probability (PDP) of SPADs as a function of wavelength of incident photons for different SPAD excess bias [79]. A high sensitivity for photons in the visible spectrum can be observed.

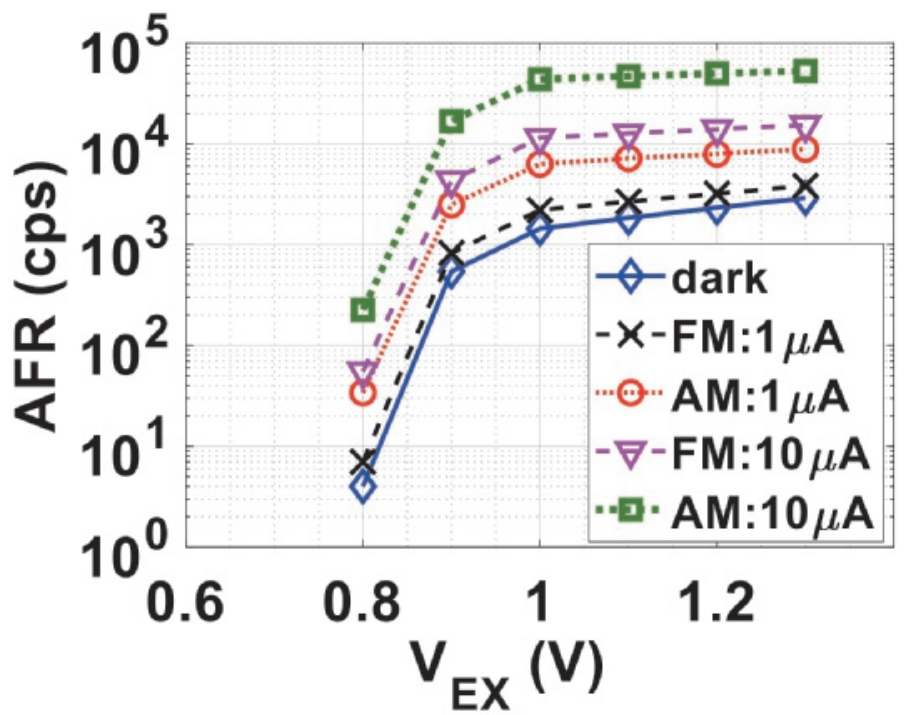

Figure 6.9: Avalanche firing rate (AFR) as a function of $V_{\mathrm{EX}}$ when $\mathrm{S} 1$ was biased above breakdown and illuminated with the integrated Si LED (A1). When A1 was biased using a reverse current in avalanche mode (AM), a higher AFR was obtained compared to the case when A1 was biased using a same value of forward mode (FM) DC current. In dark conditions, AFR is equivalent to DCR. 


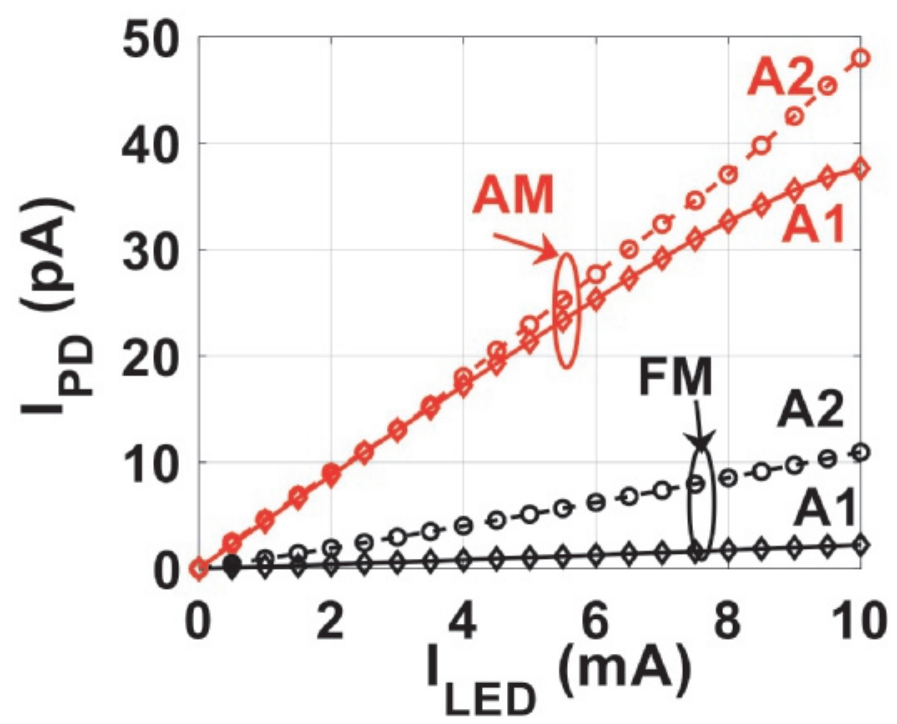

Figure 6.10: DC coupling: S1 was operated as a "conventional" PD and its short circuit current $\left(I_{\mathrm{PD}}\right.$, which is reverse current at $\left.V_{\mathrm{R}}=0\right)$ was measured when the corresponding LEDs (A1/A2) were biased in AM and FM using DC currents $\left(I_{\mathrm{LED}}\right)$. A2-S1 shows a higher $\eta_{\mathrm{CE}}\left(=I_{\mathrm{PD}} / I_{\mathrm{LED}}\right)$ than A1-S1 (Fig. 6.4).

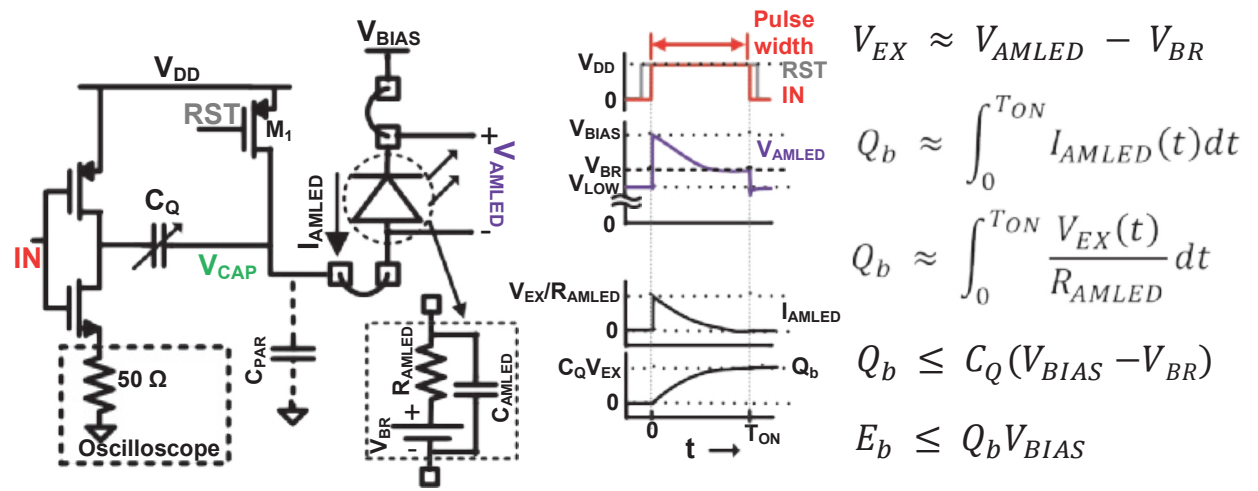

Figure 6.11: AMLED driver circuit with illustrative transients [71]. Initially, RST is set high, thus turning off the switch M1. Then IN is set high and initially voltage across an AMLED $\left(V_{\text {AMLED }}=V_{\text {BIAS }}-V_{\text {CAP }}\right)$ increases to $\sim$ $V_{\text {BIAS }}$ (assuming quenching capacitance $C_{\mathrm{Q}}$ » parasitic capacitance $C_{\mathrm{PAR}}$ ). As $V_{\mathrm{AMLED}}>V_{\mathrm{BR}}$, avalanche is triggered and the avalanche current $\left(I_{\mathrm{AMLED}}\right)$ charges $C_{\mathrm{Q}}$, thereby increasing $V_{\mathrm{CAP}}$ and reducing $V_{\mathrm{AMLED}}$. As $V_{\mathrm{AMLED}}$ approaches $V_{\mathrm{BR}}$, the avalanche is quenched. The total charge per bit through the AMLED $\left(Q_{\mathrm{b}}\right)$ is limited to $C_{\mathrm{Q}}\left(V_{\mathrm{BIAS}}-V_{\mathrm{BR}}\right)$. The energy-per-bit $\left(E_{\mathrm{b}}\right)$ is then limited to $Q_{\mathrm{b}} V_{\text {BIAS. }} I_{\text {AMLED }}$ is directly measured on a Keysight DSO-X $3024 \mathrm{~A}$ oscilloscope. In our setup, $Q_{\mathrm{b}}$ and therefore $E_{\mathrm{b}}$ could be tuned by tuning $C_{\mathrm{Q}}$ and $V_{\text {BIAS }}$ (with $V_{\text {BIAS }}-V_{\text {DD }}<V_{\text {BR }}$ ). A simplified model for an AMLED is shown in the right hand side dashed area [22, 71]. 


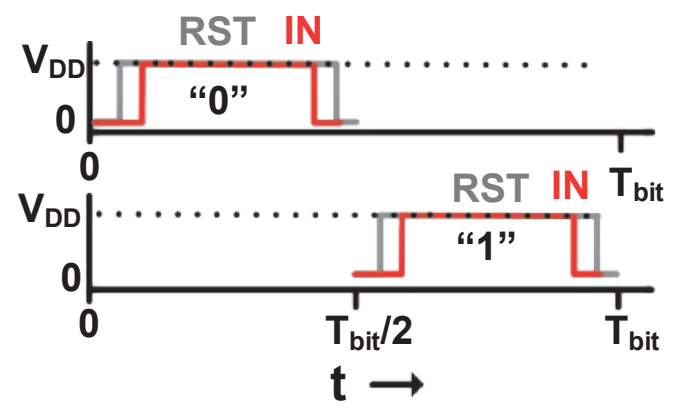

Figure 6.12: Illustrative transient waveforms of the control signals IN and RST for bit " 0 " and for bit " 1 " when data were modulated using a two-level PPM. $T_{\text {bit }}=1 / f_{\mathrm{S}}$ is the bit duration time where $f_{\mathrm{S}}$ is the data rate. For bit " 0 ", photons are transmitted in the first half of $T_{\text {bit, }}$, whereas for bit " 1 ", photons are transmitted in second half.

BER than A1-S1 because of the higher $\eta_{\mathrm{CE}}$ (see Fig. 6.10). At higher $f_{\mathrm{S}}, T_{\mathrm{ON}}$ reduces (Fig. 6.11) and therefore $Q_{\mathrm{b}}$ decreases [71]; a lower $Q_{\mathrm{b}}$ reduces the amount of photons per bit $\left(P_{\mathrm{b}}\right)$ and thereby increases the BER.

In AM, a much lower BER is obtained compared to FM operation. At higher $f_{\mathrm{S}}, \mathrm{A} 2$ shows a lower BER also because of its lower resistance $(\sim 25$ $\Omega)$ than $\mathrm{A} 1(\sim 850 \Omega)$ : for a fixed $T_{\mathrm{ON}}$, a low resistance results in a high $Q_{\mathrm{b}}$ and consequently a high $P_{\mathrm{b}}$, therefore a low BER [71]. Fig. 6.16 shows the measured BER of the optical link as the $E_{\mathrm{b}}$ through the LEDs was varied
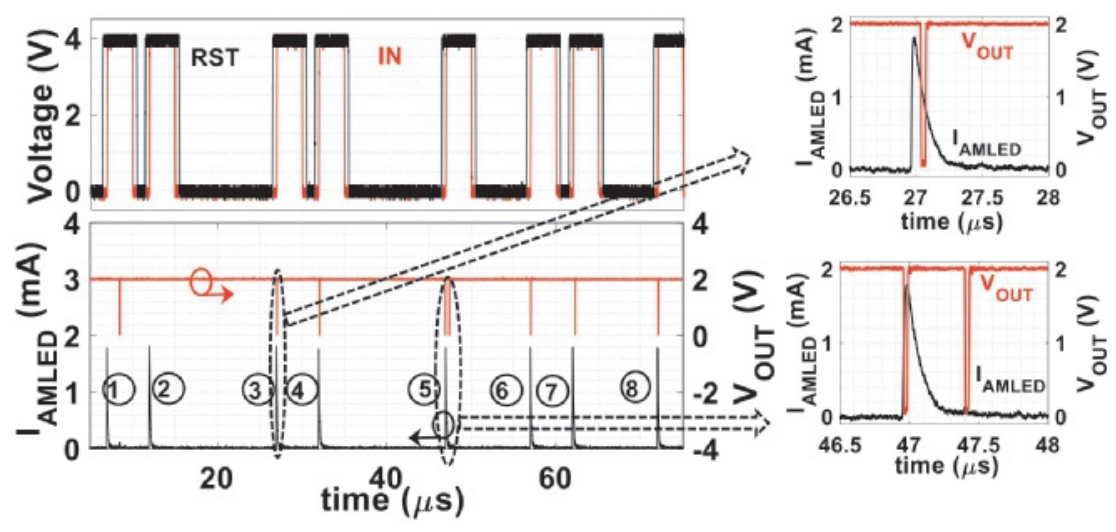

Figure 6.13: For A1-S1, $V_{\mathrm{BIAS}}=20 \mathrm{~V}, V_{\mathrm{DD}}=4 \mathrm{~V}, \mathrm{C}_{\mathrm{Q}}=100 \mathrm{pF}, f_{\mathrm{S}}=100$ kbps, $V_{\mathrm{EX}, \mathrm{SPAD}}=1 \mathrm{~V}$ : (Left) Example of measured transients of the control signals IN and RST (top); measured $I_{\text {AMLED }}$ and SPAD QRC output ( $\left.V_{\text {OUT }}\right)$ in response to those control signals (bottom). For marked bit 1 , SPAD is triggered after some delay whereas SPAD is not triggered for bit 2 causing a bit-error. For bits 3,4,6,7 and 8, SPAD is triggered when AMLED is triggered. For bit 5, SPAD is triggered multiple times. (Right : a zoomed response of $I_{\mathrm{AMLED}}$ and $V_{\mathrm{OUT}}$ (scales enhanced for clarity)). 


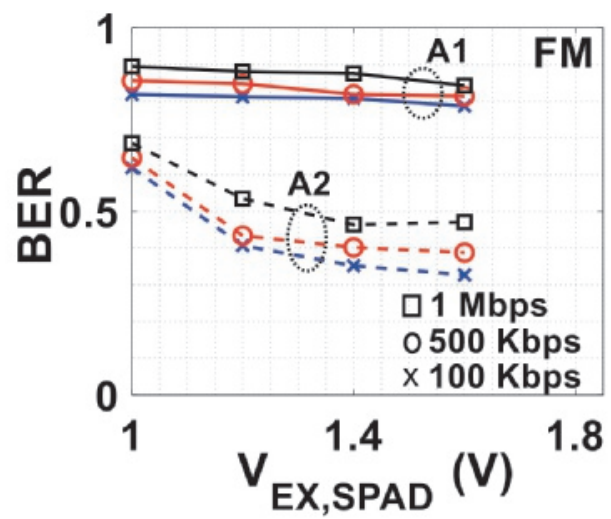

Figure 6.14: Measured BER vs excess bias of $\operatorname{SPAD}\left(V_{\mathrm{EX}, \mathrm{SPAD}}\right)$ for different $f_{S}$ with LEDs $(A 1, A 2)$ operated in FM (driver circuit of Fig. 11 with cathode and anode of the LEDs interchanged). Operating conditions of the driver circuit: $V_{\mathrm{BIAS}}=4 \mathrm{~V}, V_{\mathrm{DD}}=4 \mathrm{~V}$ and $C_{\mathrm{Q}}=220 \mathrm{pF}$. A2-S1 shows a lower BER because of a higher $\eta_{C E}$ (Figs. 6.4 and 6.10). BER vs $E_{\mathrm{b}}$ is shown in Fig. 6.16.

for several $f_{\mathrm{S}}$ in FM and AM. In AM, A2 requires $\sim 2 \mathrm{x}$ lower $E_{\mathrm{b}}$ than $\mathrm{A} 1$ for a similar BER which demonstrates the advantages of our low $V_{\text {BR }}$ AMLED design. The bare BER performance measured here can be improved using (e.g. Hamming) error-correction techniques, requiring a limited number of (low voltage) logic gates.

These results demonstrate that SPADs combined with AMLEDs are

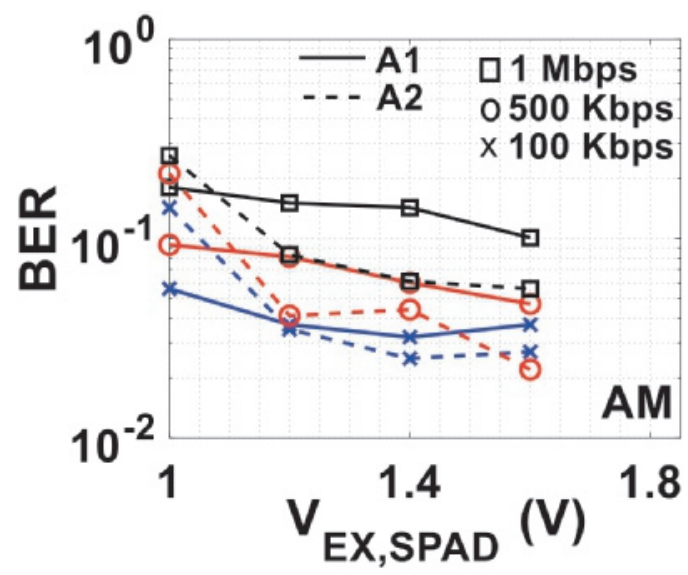

Figure 6.15: Measured BER vs $V_{\mathrm{EX}, S \mathrm{SPD}}$ for different $f_{\mathrm{S}}$ when the LEDs are operated in AM. Note that $y$-axis is on a different scale than in Fig. 6.14. Operating conditions of the driver circuit (Fig. 6.11): $V_{\text {BIAS }}=20 \mathrm{~V}$ for A1 and $9 \mathrm{~V}$ for $\mathrm{A} 2, V_{\mathrm{DD}}=4 \mathrm{~V}$ and $C_{\mathrm{Q}}=220 \mathrm{pF}$. A much lower BER is obtained for AMLED-SPAD combination than for an FMLED-SPAD combination (Fig. 6.14). BER vs $E_{\mathrm{b}}$ is shown in Fig. 6.16. 

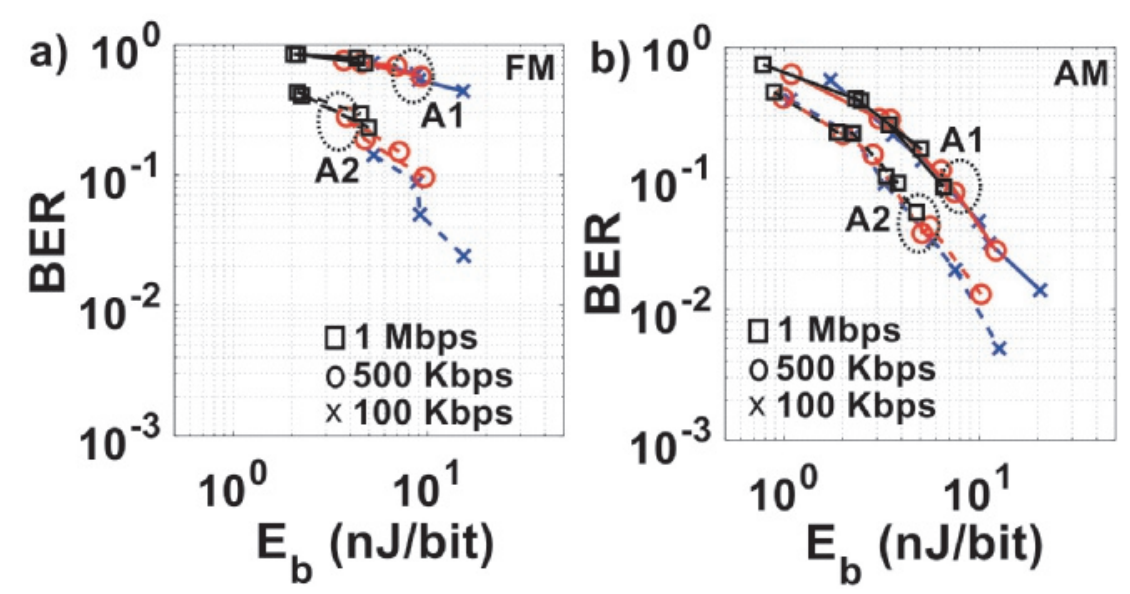

Figure 6.16: Measured BER of the optical links as a function of $Q_{b}$ for (a) FM (b) AM LED operation; $Q_{b}$ was varied by varying $C_{Q}$ and $V_{\text {BIAS }}$ for several $f_{\mathrm{S}}$. For both LEDs (A1,A2), BER reduces for higher $Q_{\mathrm{b}}$ because of higher $P_{\mathrm{b}}$. At similar $Q_{\mathrm{b}}, \mathrm{A} 2$ shows a lower BER due to a higher $Q_{\mathrm{b}}, P_{\mathrm{b}}$ and $\eta_{C E}$. Similar BER were measured across all data rates for similar $Q_{b}$. $V_{\mathrm{EX}, \mathrm{SPAD}}=1.2 \mathrm{~V}$ for all measurements.

attractive for monolithically integrated optocouplers. The performance of the system has been measured up to $1 \mathrm{Mbps}$. The AMLEDs are expected to reach a high modulation speed; $10 \mathrm{Mbps}$ using AMLEDs in this technology was shown in [71]. The designed SPADs in this technology require a deadtime of about 100 ns for a low afterpulsing probability [79]. This would limit the speed of SPADs up to $\sim 10 \mathrm{Mbps}$. Therefore, the maximum expected single channel data rate of the proposed system is $\sim 10 \mathrm{Mbps}$.

\subsection{Conclusions}

In this work, for the first time, we have successfully demonstrated data communication using a monolithically integrated optical link in a standard CMOS technology without any post processing. The optical link was implemented using Si AMLEDs and SPADs. The advantages of AMLEDs over forward biased Si LEDs were shown. The coupling efficiency of LEDs with Si PDs was improved by a novel AMLED design. Poor quantum efficiency of AMLEDs was compensated by the use of highly sensitive SPADs. The energy consumption of the system was minimized by the design of novel AMLEDs and low power driver circuits. The results show strong potential of AMLEDs-SPADs combination for monolithic optical links. 
Figure 6.17: Application example: IEEE logo (8-bit resolution) transmitted through our optical link A1-S1 (in AM) at 1 Mbps. From the received bits, image was reconstructed without any error-correction. Bits which could not be resolved from SPAD output were estimated as 0 or 1 randomly using uniform distribution. 


\section{Transmitted}

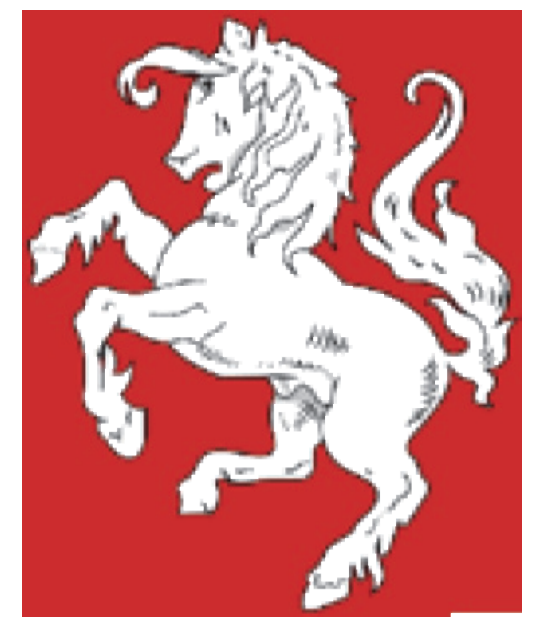

\section{Received}

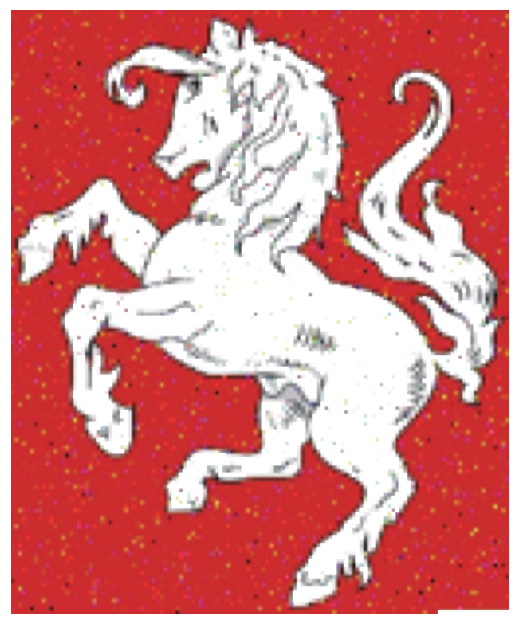

Figure 6.18: Application example: Flag of Twente transmitted through our optical link A1-S1 (in AM) at 1 Mbps. 


\section{CHAPTER}

\section{CONCLUSIONS AND RECOMMENDATIONS}

This chapter summarizes the most important conclusions of this thesis. Recommendations for future work are also discussed.

\section{Chapter 2}

This chapter focused on the Random Telegraph Signal (RTS) phenomena in diode avalanche currents. RTS phenomena were shown to determine the steep current-voltage $(I-V)$ dependency in avalanche. Using a time domain analysis (TDA), the statistical properties of these RTS phenomena were determined. An accurate experimental definition of breakdown voltage $\left(V_{\mathrm{BR}}\right)$ was proposed based on the RTS analysis. Further, the value of the self-sustaining avalanche current in diodes was parametrically determined which can be used for an accurate design of quenching-and-recharge circuits (QRCs) for SPADs. It is expected that the proposed approach can significantly increase count rates while reducing afterpulsing. The advantages of the accurate design of QRCs were discussed with circuit simulations.

This work can be extended by an experimental demonstration of the proposed accurate QRCs.

\section{Chapter 3}

In this chapter, the RTS phenomena of avalanche diodes comprising ultrashallow junctions, fabricated using a pure boron technology, were analyzed using the TDA method presented in chapter 2. From these results, the device size dependency on the RTS parameters (amplitude and mean ontime fraction) was discussed. The amplitude of the RTSs was shown to increase with the size of the diode which was explained by the spreading 
of avalanche of a single defect to more defects in larger diodes. Two different types of RTSs were observed in these diodes: a "decaying" and a "constant" amplitude type and a comprehensive analysis of both RTSs was done. It was proposed that the "decaying" amplitude RTSs are caused by defects with a relatively high thermal impedance. By modeling these RTS phenomena and using the TDA, some of the defect parameters were estimated. The ohmic series resistances of these defects were estimated to be hundreds of ohms to a few $\mathrm{k} \Omega$. The effective diameter of the defects was estimated to be in the order of a few $\mu \mathrm{m}$. Further, it was argued that the "decaying" amplitude RTSs are caused by relatively small (effective) area defects and the "constant" amplitude RTSs by relatively large area defects.

For future work, measurements on low defect density diodes would give additional information about the properties of the individual defects causing RTSs. An ideal device for such a study would be a diode with defects having significantly distinct breakdown voltages. Such devices were available in early Si technologies possibly because of the process variation. The processing is relatively more uniform in modern CMOS technologies and therefore such devices need to be explicitly designed. A possible approach would be the use of junctions with different breakdown voltages in a single device. Geometric variations (e.g. a sharp edge at one location of a device) can also result in defects having different breakdown voltages. Simultaneous optical measurements and TDA can be used to completely characterize defects in terms of their geometry and electrical parameters.

High speed optical measurements such as those used in photo emission micrography (PEM) can be used to investigate the propagation of RTSs in time domain. For the two types of RTSs, another hypothesis was also discussed but could not be verified: the "decaying" RTSs are caused by the defects located at the periphery of a diode whereas "constant" RTSs are caused by the defects in the active area of a diode. This hypothesis can be verified with high speed and highly sensitive optical measurements as well.

\section{Chapter 4}

This chapter discussed the data transmission capabilities of Si avalanche mode LEDs (AMLEDs) fabricated in CMOS technologies. The data were modulated using a pulse position modulation scheme, and the bit-errorrate (BER) and the jitter in the transmitted data were measured. The results were correlated to the multiplication noise $\left(\sigma_{M}\right)$ and the leakage current of the AMLEDs. The $\sigma_{M}$ of these AMLEDs were shown to increase with the size of the AMLEDs because of more defects in larger diodes. It was shown that AMLEDs with a higher $\sigma_{M}$ display a lower BER and jitter. Through temperature and optical excitations, it was demonstrated that an 
elevated leakage current improves the triggering rate of avalanche, thereby reducing the BER and jitter. Based on the outcome of this research, design recommendations to improve the speed of the AMLEDs were discussed.

In this chapter, a three terminal AMLED device structure has been proposed for high speed but not yet verified through measurements. The demonstration of such a device structure could be important for design of high speed optical links.

It was observed that diodes with an STI interface achieve a higher speed, however have a lower leakage current. A possible explanation for this could be that defects formed near the STI do not contribute to the leakage current, but do affect the RTS behavior. However, the effect of an STI interface on the leakage current could not be investigated due to the lack of diodes with a different geometry. From the $I-V$ characteristics of diodes with different sizes, the contribution of an STI interface on the leakage current and RTS behavior can be extracted in a future work.

\section{Chapter 5}

A low power monolithically integrated optical transmitter using AMLEDs was demonstrated in a standard $140 \mathrm{~nm}$ SOI CMOS technology. A novel self-quenched driver circuit was proposed which resolves many physics issues related to power dissipation and process, voltage and temperatures (PVT) variations of an AMLED. This work successfully demonstrated a low power wide spectrum optical transmitter in a CMOS technology which can be integrated with standard Si PDs. It further reinforced the promise of enabling AMLEDs as light sources in silicon CMOS technologies for monolithic integration of optocouplers.

\section{Chapter 6}

In this chapter, for the first time, we have successfully demonstrated data communication using a monolithically integrated optical link in a standard CMOS technology without any post processing. The optical link was implemented using Si AMLEDs and SPADs. The advantages of AMLEDs over forward biased Si LEDs were shown. The coupling efficiency of LEDs with Si PDs was improved by a novel AMLED design. Poor quantum efficiency of AMLEDs was compensated by the use of highly sensitive SPADs. The energy consumption of the system was minimized by the design of novel AMLEDs and low power driver circuits. A comparison between an optical link employing an forward biased Si LEDs and SPAD, i.e. FMLED-SPAD structure with that of an AMLED-SPAD structure was also done and the advantages of AMLEDs over FMLEDs was clearly demonstrated. The results show strong potential of AMLEDs-SPADs combination for monolithic optical links. 
The achieved coupling efficiency between the AMLEDs and the PDs $\left(\eta_{\mathrm{CE}}\right)$ can be improved by the use of waveguiding structures, e.g. metallic waveguides as shown in [41]. A higher $\eta_{C E}$ would result in a lower energyper-bit $\left(E_{\mathrm{b}}\right)$ for an optical link.

\section{General recommendations}

The SPADs need to be designed to achieve a low dark count rate (DCR) for a large dynamic range of an optical link. For a low DCR, the depletion region of a SPAD should be located as far as possible from the oxide interfaces. The oxide interfaces induce defects in the depletion region that degrade the quality of SPADs. These interface defects can be reduced by the use of guard rings surrounding SPADs. A downside is that a guard ring reduces the $\eta_{\mathrm{CE}}$ because of the high absorption coefficient of Si. A lower $\eta_{\mathrm{CE}}$ increases the $E_{\mathrm{b}}$ requirement at the transmitter. Therefore, an optimum value for the width of the guard ring would be required for a low DCR and a low $E_{\mathrm{b}}$.

In this project, the devices consisted of shallow junctions. Integrated optocouplers however require lateral coupling of light and consequently deeper junctions are preferred for SPADs to reduce the impact of back-end oxide interfaces.

The speed of AMLEDs is expected to increase at smaller technology nodes because of a higher number of defects and smaller distances from the oxide interfaces. Therefore, AMLEDs have an intrinsic advantage with the technology downscaling. However, the same scaling makes the design of SPADs in those technologies more challenging. The design of SPADs in smaller technology nodes is currently under active research.

The maximum operating speed of SPADs is expected to be about tens of Mbps. The use of time-interleaved structures can increase the achievable data rate of an optical link at the expense of power, area and more complicated driver and readout circuits.

Some technologies offer SiGe junctions; SiGe PDs have a higher responsivity at infrared wavelengths. Employing the SiGe junctions could also be an attractive way to implement optical links in those technologies.

Reliability and degradation analysis of systems implemented using avalanche diodes were not addressed in the scope of this thesis and are an important topic for future research as well.

\section{Original contributions of this thesis}

- The $I-V$ characteristics of the diodes operating in avalanche was explained using the RTS phenomena [68]. Using a time domain analysis, an accurate definition of experimental breakdown voltage was provided. 
- Two types of RTSs have been shown in the avalanche current of diodes [69]. These RTSs were explained to be caused by the different thermal impedance of defects in the silicon. Based on a physics based model, the properties of the defects (ohmic series resistance and effective diameter) were estimated. The effective diameter of the defects were also estimated using optical measurements and a correlation with the TDA measurements was shown. The proposed model and analysis method can be used to estimate the properties of the defects for diodes in any technology.

- The dependency of the RTS parameters on the size of the diode was discussed [69]. For a larger diode, the increasing amplitude of the RTSs was explained by the spreading of avalanche of a single defect to more defects. The multiplication noise also increases for larger diodes because of an increasing number of defects.

- The application of the analysis of RTS phenomena for accurate design of quenching-and-recharge circuits (QRCs) was discussed [68]. It is expected that accurate QRCs can increase the count rates, while reducing afterpulsing effects in SPADs.

- Data transmission capabilities of AMLEDs were investigated and the results were correlated to physically measurable multiplication noise and leakage current of AMLEDs [70]. It was demonstrated that a large number of defects and a high leakage current are required for high speed AMLEDs.

- The AMLEDs were integrated with low power driver circuits to implement a monolithic optical transmitter in a standard $140 \mathrm{~nm}$ CMOS technology [71]. Using extensive electrical and optical measurements, the robustness of this transmitter to the process, voltage, temperature and operating conditions was shown. The light emitted by the transmitter was detected using a laterally integrated photodetector, hence demonstrating optocoupling in CMOS. From the experimental results, the IQE of Si AMLEDs was estimated to be in the order of $10^{-5}$.

- Finally, for the first time, data communication using monolithically integrated optocouplers in a standard CMOS technology without any post-processing was demonstrated [72]. A data rate of few Mbps with energy consumption of a few $\mathrm{nJ} /$ bit was shown. 



\section{BIBLIOGRAPHY}

[1] J. Clarke, "An introduction to communications with optical carriers,", Students' Quarterly Journal, vol. 36, no. 144, pp. 218-222, 1966, 10.1049/sqj.1966.0040.

[2] R. N. Hall, G. E. Fenner, J. D. Kingsley, T. J. Soltys and R. O Carlson, "Coherent Light Emission From GaAs Junctions,", American Physical Society, vol. 9, no. 9, pp. 366-368, 1962, 10.1103/PhysRevLett.9.366.

[3] H. Kroemer, "A proposed class of hetero-junction injection lasers,", Proc. IEEE, vol. 51, no. 12, pp. 1782-1783, 1963, 10.1109/PROC.1963.2706.

[4] Z. I. Alferov, "The double heterostructure concept and its applications in physics, electronics, and technology,", Rev. Mod. Phys., vol. 73, no. 3, pp. 767-782, 2001, 10.1103/RevModPhys.73.767.

[5] K. C. Kao and G. A. Hockham, "Dielectric-fibre surface waveguides for optical frequencies,", Proceedings of the Institution of Electrical Engineers, vol. 113, no. 7, pp. 1151-1158, 1966, 10.1049/piee.1966.0189.

[6] G. E. Moore, "Cramming more components onto integrated circuits,", Electronics, vol. 38, no. 8, pp. 114, 1965.

[7] G. E. Moore, "Progress in digital integrated electronics,", International Electron Devices Meeting, pp. 11-13, 1975.

[8] G. E. Moore, "No exponential is forever: but "Forever" can be delayed!,", IEEE International Solid-State Circuits Conference, vol. 1, pp. 20-23, 2003, 10.1109/ISSCC.2003.1234194.

[9] B. Razavi, Design of Integrated Circuits for Optical Communications, McGraw-Hill (2003).

[10] I. G. Akmenkalns, R. J. Wilfinger and A. D. Wilson, "Four terminal electro-optical logic device,", US patent 3,417,249, 1963.

[11] "IL4208 Optocoupler, phototriac output, high dV/dt, low input current,", Product dataheet, Vishay Semiconductors.

[12] S. M. Sze and K. K. Ng, "Physics of semiconductor devices," Wiley, 2007.

[13] S. Dutta, V. Agarwal, R.J.E. Hueting, J. Schmitz and A.J. Annema, "Monolithic optical link in silicon-on-insulator CMOS technology," Opt. Express, vol. 25, no. 5, pp. 5440-5456, 2017, 10.1364/OE.25.005440.

[14] R. Newman, "Visible light from a Silicon p-n junction,", Phys. Rev., vol. 100, no. 2, pp. 700-703, 1955, 10.1103/PhysRev.100.700. 
[15] L. W. Snyman and M. d. Plessis and H. Aharoni, "Injection-AvalancheBased $\mathrm{n}^{+}$pn Silicon Complementary Metal Oxide Semiconductor Light Emitting Device (450 - $750 \mathrm{~nm}$ ) with 2-Order-of-Magnitude Increase in Light Emission Intensity," Jpn. J. Appl. Phys., vol. 46, no. 4B, pp. $2474-$ 2480, 2007, 10.1143/JJAP.46.2474.

[16] L. W. Snyman, H. Aharoni, A. Biber, A. Bogalecki, L. Canning, M. du Plessis and P. Maree, "Optical sources, integrated optical detectors, and optical waveguides in standard silicon CMOS integrated circuitry," Proc. SPIE, vol. 3953, pp. 3953-3953-17, 2000, 10.1117/12.379613.

[17] B.P. van Drieënhuizen and R.F. Wolffenbuttel, "Optocoupler based on the avalanche light emission in silicon,", Sensors and Actuators A: Physical, vol. 31, no. 1, pp. 229-240, 1992, 10.1016/0924-4247(92)80110-O.

[18] K. Xu, W. Sun, K.A. Ogudo, L. W. Snyman, J-L. Polleux, Q. Yu and G. $\mathrm{Li}$, "Silicon Avalanche based Si LEDs and their potential integration into CMOS and RF bipolar technology," ISBN: 978-953-51-1730-8, InTech, 2014, 10.5772/58505.

[19] A. S. Kamath, N. Bhardwaj and K. Soundarapandian, "Understanding failure mode in isolators,", Texas Instruments Application Note, 2015.

[20] K. Gingerich and C. Sterzik, "The ISO72x Family of High-Speed Digital Isolators," Application Report SLLA198, Texas Instruments (2006).

[21] E. F. Schubert, "Light-emitting diodes," Cambridge university press, 2006.

[22] S. Cova, M. Ghioni, A. Lacaita, C. Samori and F. Zappa, "Avalanche photodiodes and quenching circuits for single-photon detection," , Appl. Opt., vol. 35, no. 12, pp. 1956-1976, 1996, 10.1364/AO.35.001956.

[23] M. W. Fishburn, "Fundamentals of CMOS single photon avalanche diodes," Ph.D. dissertation, Dept. Elect. Eng., Delft Univ. Technol., Delft, The Netherlands, (2012).

[24] P. P. Webb and R. J. McIntyre, "Single photon detection with avalanche photodiodes," Bull. Amer. Phys. Soc. II, vol. 15, pp. 813, 1970.

[25] R. J. McIntyre, "On the avalanche initiation probability of avalanche diodes above the breakdown voltage," IEEE Trans. Elec. Dev., vol. 20, no. 7, pp. 637-641, 1973, 10.1109/T-ED.1973.17715.

[26] S. Cova, A. Longoni and A. Andreoni, "Towards picosecond resolution with single - photon avalanche diodes," Review of Scientific Instruments, vol. 52, no. 3, pp. 408-412, 1981, 10.1063/1.1136594.

[27] Philip A. Ekstrom, "Triggered - avalanche detection of optical photons," Jrnl. of Appl. Phys., vol. 52, no. 11, pp. 6974-6979, 1981, 10.1063/1.328654.

[28] R. J. McIntyre, "Recent developments in silicon avalanche photodiodes," Measurement, vol. 3, no. 4, pp. 146-152, 1985, 10.1016/0263-2241(85)900247.

[29] M. Ghioni, A. Gulinatti, I. Rech, F. Zappa and S. Cova, "Progress in Silicon Single-Photon Avalanche Diodes," IEEE Journal of Selected 
Topics in Quantum Electronics, vol. 13, no. 4, pp. 852-862, 2007, 10.1109/JSTQE.2007.902088.

[30] M. Ghioni, S. Cova, A. Lacaita and G. Ripamonti, "New silicon epitaxial avalanche diode for single-photon timing at room temperature," Electronics Letters, vol. 24, no. 24, pp. 1476-1477, 1988, 10.1049/el:19881007.

[31] M. Marisaldi, P. Maccagnani, F. Moscatelli, C. Labanti, F. Fuschino, M. Prest, A. Berra, D. Bolognini, M. Ghioni, I. Rech, A. Gulinatti, A. Giudice, G. Simmerle, D. Rubini, A. Candelori and S. Mattiazzo, "Single Photon Avalanche Diodes for space applications," IEEE Nuclear Science Symposium Conference Record, pp. 129-134, 2011, 10.1109/NSSMIC.2011.6154465.

[32] D. Bronzi, F. Villa, S. Tisa, A. Tosi and F. Zappa, "SPAD Figures of Merit for Photon - Counting, Photon - Timing, and Imaging Applications: A Review," IEEE Sensors Journal, vol. 16, no. 1, pp. 3-12, 2016, 10.1109/JSEN.2015.2483565.

[33] C. Bruschini, H. Homulle, E. Charbon, "Ten years of biophotonics singlephoton SPAD imager applications: retrospective and outlook," Proc. SPIE, vol. 10069, pp. 10069-10069-21, 2017, 10.1117/12.2256247.

[34] V. Mitov and C. K. Tee, "Gate Drive Optocoupler Provides Robust Insulation in IGBT Destructive Tests," Proceedings of PCIM Europe 2015; International Exhibition and Conference for Power Electronics, Intelligent Motion, Renewable Energy and Energy Management, pp. 1-7, 2015.

[35] B. Murari, F. Bertotti and G. A. Vignola, "Smart power ICs," Springer, 1996.

[36] P. Wessels, M. Swanenberg, H. van Zwol, B. Krabbenborg, H. Boezen, M. Berkhout, and A. Grakist, "Advanced BCD technology for automotive, audio and power applications," Solid-State Electronics, vol. 51, no. 2, pp. 195-211, 2007.

[37] “Digital isolator design guide," Developer's guide, SLLA284A, Texas Instruments (2014).

[38] D. A. B. Miller, "Device requirements for optical interconnects to silicon chips," Proc. IEEE, vol. 97, no. 7, pp. 1166-1185, 2009.

[39] Y. Moghe, A. Terry and D. Luzon, "Monolithic 2.5kV RMS, 1.8V - 3.3V dual-channel $640 \mathrm{Mbps}$ digital isolator in $0.5 \mu \mathrm{m}$ SOS," IEEE International SOI Conference (SOI), pp. 1-2, 2012.

[40] S. Mukherjee, A. N. Bhat, A. Shrivastava, M. Bonu, B. Sutton, J. Malakar, and N. Krishnapura, "A 500Mb/s 200pJ/b die-to-die bidirectional link with $24 \mathrm{kV}$ surge isolation and $50 \mathrm{kV} / \mu \mathrm{s}$ CMR using resonant inductive coupling in $0.18 \mu \mathrm{m}$ CMOS," IEEE International Solid-State Circuits Conference (ISSCC), pp. 434-435, 2017.

[41] B. Huang, X. Zhang, W. Wang, Z. Dong, N. Guan, Z. Zhang, and H. Chen, "CMOS monolithic optoelectronic integrated circuit for on-chip 
optical interconnection," Opt. Commun., vol. 284, pp. 3924-3927, 2011.

[42] K. G. McKay, "Avalanche Breakdown in Silicon,", Phys. Rev., vol. 94, no. 4, pp. 877-884, 1954, 10.1103/PhysRev.94.877.

[43] D. J. Rose, "Microplasmas in Silicon,", Phys. Rev., vol. 105, no. 2, pp. 413-418, 1957, 10.1103/PhysRev.105.413.

[44] B. Senitzky and J. L. Moll, "Breakdown in Silicon,", Phys. Rev., vol. 110, no. 3, pp. 612-620, 1958, 10.1103/PhysRev.110.612.

[45] Keith S. Champlin, "Microplasma Fluctuations in Silicon,", Jrnl. Appl. Phys., vol. 30, no. 7, pp. 1039-1050, 1959, 10.1063/1.1776976.

[46] A. G. Chynoweth and K. G. McKay, "Light Emission and Noise Studies of Individual Microplasmas in Silicon p-n Junctions,", Jrnl. Appl. Phys., vol. 30, no. 11, pp. 1811-1813, 1959, 10.1063/1.1735060.

[47] R. L. Batdorf, A. G. Chynoweth, G. C. Dacey and P. W. Foy, “Uniform Silicon p-n Junctions. I. Broad Area Breakdown,", Jrnl. Appl. Phys., vol. 31, no. 7, pp. 1153-1160, 1960, 10.1063/1.1736199.

[48] W. Shockley, "Problems related to p - n junctions in silicon,", Solid-State Electronics, vol. 2, no. 1, pp. 35-67, 1961, 10.1016/0038-1101(61)90054-5.

[49] R. J. McIntyre, "Theory of Microplasma Instability in Silicon,", Jrnl. Appl. Phys., vol. 32, no. 6, pp. 983-995, 1961, 10.1063/1.1736199.

[50] R. H. Haitz, A. Goetzberger, R. M. Scarlett and W. Shockley, “Avalanche Effects in Silicon p-n Junctions. I. Localized Photomultiplication Studies on Microplasmas,", Jrnl. Appl. Phys., vol. 34, no. 6, pp. 1581-1590, 1963, 10.1063/1.1702639.

[51] A. Goetzberger and B. McDonald and R. H. Haitz and R. M. Scarlett, "Avalanche Effects in Silicon p-n Junctions. II. Structurally Perfect Junctions,", Jrnl. Appl. Phys., vol. 34, no. 6, pp. 1591-1600, 1963, 10.1063/1.1702640.

[52] R. H. Haitz, "Model for the Electrical Behavior of a Microplasma,", Jrnl. Appl. Phys., vol. 35, no. 5, pp. 1370-1376, 1964, 10.1063/1.1713636.

[53] J. Kölzer, C. Boit, A. Dallmann, G. Deboy, J. Otto and D. Weinmann, "Quantitative emission microscopy,", Jrnl. Appl. Phys., vol. 71, no. 11, pp. R23-R41, 1992, 10.1063/1.350466.

[54] T. Matsudai, K. Endo, T. Ogura, T. Matsumoto, K. Uchiyama, F. Niikura and K. Koshikawa, "Direct photo emission monitoring for analysis of IGBT destruction mechanism using streak camera," , International Symposium on Power Semiconductor Devices and ICs (ISPSD), pp. 135-138, 2017, 10.23919/ISPSD.2017.7988945.

[55] R. H. Haitz, "Studies on optical coupling between silicon pn junctions,", Solid-State Electronics, vol. 8, no. 4, pp. 417-425, 1965, 10.1016/00381101(65)90119-X.

[56] F. Zappa, S. Tisa, A. Tosi and S. Cova "Principles and features of singlephoton avalanche diode arrays,", Sensors and Actuators A: Physical, vol. 
140, no. 1, pp. 103-112, 2007, 10.1016/j.sna.2007.06.021.

[57] L. J. Kabell and C. J. Pccoraro, "Silicon avalanche light sources for photographic data recording,", J. T. Tippett (ed.), Optical and Electro-optical Information Processing, Ch. 37, pp. 707-714, MIT Press, 1968.

[58] D. V. Kerns, K. Arora, S. Kurinec and W. Power, "Si diode under avalanche breakdown as a light emitting source for VLSI optical interconnect,", Proc. of The Twenty-First Southeastern Symposium on System Theory, pp. 677-680, 1989, 10.1109/SSST.1989.72553.

[59] L. W. Snyman, "Integrating Micro-Photonic Systems and MOEMS into Standard Silicon CMOS Integrated Circuitry, Optoelectronics Devices and Applications," ISBN: 978-953-307-576-1, InTech, 2011, $10.5772 / 18810$.

[60] L. W. Snyman, T. Okhai, T. Bourouina and W. Noell, "Development of on-CMOS chip micro-photonic and MOEMS systems," Proc. SPIE 7930, 2011, doi: $10.1117 / 12.873353$.

[61] A. Chatterjee and B. L. Bhuva, "Accelerated stressing and degradation mechanisms for Si-based photo emitters," IEEE Trans. Device and Materials Reliability, vol. 2, no. 3, pp. 60-64, 2002.

[62] A. Chatterjee, P. Mongkolkachit, B. L. Bhuva, and A. Verma, "All Sibased optical interconnect for interchip signal transmission," IEEE Photonics Technology Letters, vol. 15, no. 11, pp. 1663-1665, 2003.

[63] A. Chatterjee, B. L. Bhuva, and R. D. Schrimpf, "High-speed light modulation in avalanche breakdown mode for Si diodes," IEEE Electron Device Letters, vol. 25, no. 9, pp. 628-630, 2004.

[64] N. Lodha, S. Mandai, and E. Charbon, "Monolithic Integration of LEDs and SPADs in Standard CMOS Technology for Optical Joystick Application," Proceedings of International Image Sensor Workshop, 2013.

[65] Y. Wang, D. Luo, Q. Pan, L. Jing, Z. Li, and C. P. Yue, “A 60-GHz 4$\mathrm{Gb} / \mathrm{s}$ Fully Integrated NRZ-to-QPSK Fiber-Wireless Modulator," IEEE Transactions on circuits and systems-I, vol. 1, no. 11, pp. 653-663, 2017.

[66] C. Veerappan, Single-photon avalanche diodes for cancer diagnosis, Ph.D. dissertation, Delft University of Technology, The Netherlands (2016).

[67] S. Dutta, "Avalanche-mode silicon LEDs for monolithic optical coupling in CMOS technology," Ph.D. dissertation, Dept. Elect. Eng., University of Twente, Enschede, The Netherlands, (2017).

[68] V. Agarwal, A. J. Annema, S. Dutta, R. J. E. Hueting, L. K. Nanver and B. Nauta, "Random Telegraph Signal phenomena in avalanche diodes: Application to SPADs," $46^{\text {th }}$ European Solid-State Device Research Conference (ESSDERC), Lausanne, pp. 264-267, 2016, 10.1109/ESSDERC.2016.7599636.

[69] V. Agarwal, A. J. Annema, S. Dutta, R. J. E. Hueting, L. K. Nanver and B. Nauta, "Random Telegraph Signal phenomena in ultra shallow $\mathrm{p}^{+} \mathrm{n}$ 
avalanche diodes," IEEE J. Elec. Dev. Society, vol. 6, no. 1, pp. 642 - 652, 2018, 10.1109/JEDS.2018.2835153.

[70] V. Agarwal, A. J. Annema, S. Dutta, R. J. E. Hueting, L. K. Nanver and B. Nauta, "Data transmission capabilities of silicon avalanche mode light emitting diodes," IEEE Trans. Elec. Dev., vol. 65, no. 11, pp. 4883-4890, 2018, 10.1109/TED.2018.2868126.

[71] V. Agarwal, S. Dutta, A. J. Annema, R. J. E. Hueting, P. G. Steeneken and B. Nauta, "Low power wide spectrum optical transmitter using avalanche mode LEDs in SOI CMOS technology," Opt. Express, vol. 25, no. 15, pp. 16981-16995, 2017, 10.1364/OE.25.016981.

[72] V. Agarwal, S. Dutta, A. J. Annema, R. J. E. Hueting, M.-J. Lee, E. Charbon and B. Nauta, "Optocoupling in CMOS," presented at the IEEE Electron Devices Meeting (IEDM) 2018.

[73] W. G. Oldham, R. R. Samuelson and P. Antognetti, “Triggering Phenomena in Avalanche Diodes," IEEE Trans. Electron Devices, vol. 19, no. 9, pp. 1056-1060, 1972, 10.1109/T-ED.1972.17544.

[74] O. Marinov, M. J. Deen and J. A. Jimenez Tejada, "Theory of microplasma fluctuations and noise in silicon diode in avalanche breakdown," J. Appl. Phys., vol. 101, no. 6, pp. 064515-064515-21, 2007, 10.1063/1.2654973.

[75] E. Charbon, "Single-photon imaging in complementary metal oxide semiconductor processes,", Phil. Trans. R. Soc. A, 372, 20130100, 2014, 10.1098/rsta.2013.0100.

[76] S. Dutta, V. Agarwal, R.J.E. Hueting, J. Schmitz and A.J. Annema, "Monolithic optical link in silicon-on-insulator CMOS technology," Opt. Express, vol. 25, no. 5, pp. 5440-5456, 2017, 10.1364/OE.25.005440.

[77] J. A. Richardson, E. A. G. Webster, L. A. Grant and R. K. Henderson, "Scaleable single-photon avalanche diode structures in nanometer CMOS technology," IEEE Trans. Electr. Dev., vol. 58, no. 7, pp. 2028-2035, 2011, 10.1117/12.884097.

[78] C. Niclass, C. Favi, T. Kluter, F. Monnier and E. Charbon, "Single-photon synchronous detection," IEEE J. of Solid-state circuits, vol. 44, no. 7, pp. 1977-1989, 2009, 10.1109/JSSC.2009.2021920.

[79] M. J. Lee, P. Sun, and E. Charbon, "A first single-photon avalanche diode fabricated in standard SOI CMOS technology with a full characterization of the device," Opt. Express, vol. 23, no. 10, pp. 13200-13209, 2015, 10.1364/OE.23.013200.

[80] M. S. Tyagi, "Introduction to Semiconductor Materials and Devices," (Wiley, 1991).

[81] S. Machlup, "Noise in Semiconductors: Spectrum of a Two-Parameter Random Signal," J. Appl. Phys., vol. 25, no. 3, pp. 341-343, 1954, 10.1063/1.1721637.

[82] M. A. Karami, L. Carrara, C. Niclass, M. Fishburn and E. Charbon, “RTS 
noise characterization in Single-Photon Avalanche Diodes," IEEE Elec. Dev. Lett., vol. 31, no. 7, pp. 692-694, 2010, 10.1109/LED.2010.2047234.

[83] Y. Yuzhelevski, M. Yuzhelevski, and G. Jung, "Random telegraph noise analysis in time domain," Review of Scientific Instruments, vol. 71, no. 4, pp. 1681-1688, 2000, 10.1063/1.1150519.

[84] J. S. Kolhatkar, "Steady-state and cyclo-stationary RTS noise in MOSFETS," Ph.D. dissertation, Dept. Elect. Eng., Univ. of Twente, The Netherlands, (2005).

[85] R. J. McIntyre, "Multiplication noise in uniform avalanche diodes," IEEE Trans. Electron Devices, vol. ED-13, no. 1, pp. 164-168, 1966, 10.1109/TED.1966.15651.

[86] U. Madhow, "Fundamentals of Digital Communication,", (Cambridge Univ. Press, 2008).

[87] Y. Kang, H. X. Lu, Y.-H. Lo, D. S. Bethune and W. P. Risk, “Dark count probability and quantum efficiency of avalanche photodiodes for singlephoton detection," Appl. Phys. Lett., vol. 83, no. 14, pp. 2955-2957, 2003, 10.1063/1.1616666.

[88] A. Gallivanoni, I. Rech and M. Ghioni, "Progress in Quenching Circuits for Single Photon Avalanche Diodes," IEEE Trans. on nuclear science, vol. 57, no. 6, pp. 3815-3826, 2010, 10.1109/TNS.2010.2074213.

[89] R. Mita, G. Palumbo and P. G. Fallica, "Accurate model for single-photon avalanche diodes," IET Circuits, Devices and Systems, vol. 2, no. 2, pp. 207-212, 2008, 10.1049/iet-cds:20070180.

[90] R. J. E. Hueting, A. Heringa, B. K. Boksteen, S. Dutta, A. Ferrara, V. Agarwal and A. J. Annema, "An improved analytical model for carrier multiplication near breakdown in diodes," IEEE Trans. Electr. Dev., vol. 64, no. 1, pp. 264-270, 2017, 10.1109/TED.2016.2630083.

[91] O. Marinov, M. J. Deen, V. Loukanov and V. Velikov, "The low frequency noise in reverse biased rectifier diodes,". IEEE Trans. Electron Devices, vol. 49, no. 1, pp. 184-187, 2002, 10.1109/16.974768.

[92] P. G. Neudeck, W. Huang and M. Dudley, “Breakdown degradation associated with elementary screw dislocations in $4 \mathrm{H}-\mathrm{SiC} \mathrm{p}+\mathrm{n}$ junction rectifiers," Solid-State Electronics, vol. 42, no. 12, pp. 2157-2164, 1998, 10.1016/S0038-1101(98)00211-1.

[93] L. K. Nanver, A. Sammak, A. Sakic, V. Mohammadi, J. Derakhshandeh, K. R. C. Mok, L. Qi, N. Golshani, T. M. L. Scholtes and W. B. de Boer, "Applications of PureB and PureGaB ultrashallow junction technologies," IEEE 11th International Conf. on Solid-State and Integrated Circuit Tech., pp. 1-4, 2012, 10.1109/ICSICT.2012.6467697.

[94] L. Qi, K. R. C. Mok, E. Charbon, L. K. Nanver, M. Aminian and E. Charbon, "UV-sensitive low dark-count PureB single-photon avalanche diode," IEEE Trans. Electron Devices, vol. 61, no. 11, pp. 3768-3774, 2014, 
10.1109/ICSENS.2013.6688603.

[95] L. K. Nanver, L. Qi, V. Mohammadi, K. R. M. Mok, W. B. de Boer, N. Golshani, A. Sammak, T. L. M. Scholtes, A. Gottwald, U. Kroth and F. Scholze, "Robust UV / VUV / EUV PureB Photodiode Detector Technology With High CMOS Compatibility," IEEE Jrnl. of Sel. Topics in Quantum Electronics, vol. 20, no. 6, pp. 306-316, 2014, 10.1109/JSTQE.2014.2319582.

[96] D. A. Ramirez and M. M. Hayat and G. J. Rees and X. Jiang and M. A. Itzler, "New perspective on passively quenched single photon avalanche diodes: effect of feedback on impact ionization," Opt. Express, vol. 20, no. 2, pp. 1512-1529, 2012, 10.1364/OE.20.001512.

[97] M. J. Kirton and M. J. Uren, "Noise in solid state microstructures: A new perspective on individual defects, interface states and low frequency (1/f) noise," Adv. in Phys., vol. 38, no. 4, pp. 367-468, 1989, $10.1080 / 00018738900101122$.

[98] S. M. Sze and W. Shockley, "Unit-cube expression for space-charge resistance," The Bell System Tech. Jrnl., vol. 46, no. 5, pp. 837-842, 1967, 10.1002/j.1538-7305.1967.tb01716.x.

[99] M. Schroter and A. Chakravorty, "Compact hierarchical bipolar transistor modeling with HICUM," Singapore: World Scientific, 2010, ser. International Series on Advances in Solid State Electronics and Technology.

[100] C. R. Crowell and S. M. Sze, “Temperature dependence of avalanche multiplication in semiconductors," Appl. Phys. Lett., vol. 9, no. 6, pp. 242-244, 1966, 10.1063/1.1754731.

[101] C. Y. Chang, S. S. Chiu and L. P. Hsu, "Temperature dependence of breakdown voltage in silicon abrupt p-n junctions," IEEE Trans. Electron Devices, vol. 18, no. 6, pp. 391-393, 1971, 10.1109/T-ED.1971.17210.

[102] A. Spinelli and A. L. Lacaita, "Physics and numerical simulation of single photon avalanche diodes," IEEE Trans. Electron Devices, vol. 44, no. 11, pp. 1931-1943, 1997, 10.1109/16.641363.

[103] T. L. Bergman, A. S. Lavine, F. P. Incropera and David P. DeWitt, "Fundamentals of Heat and Mass Transfer," Wiley, 2007.

[104] K. Xu, K. A. Ogudo, J.-L. Polleux, C. Viana, Z. Ma, Z. Li, Q. Yu, G. $\mathrm{Li}$ and L. W. Snyman, "Light Emitting Devices in Si CMOS and RF Bipolar Integrated Circuits," LEUKOS, vol. 12, no. 4, pp. 203-212, 2016, 10.1080/15502724.2015.1134333.

[105] K. Xu, L. W. Snyman, J.-L. Polleux, K. A. Ogudo and C. Viana, Q. Yu and G. P. Li, "Silicon LEDs toward high frequency on-chip link," Optik, vol. 127, pp. 7002-7020, 2016, 10.1016/j.ijleo.2016.05.025.

[106] K. Gingerich and C. Sterzik, "The ISO72x Family of High-Speed Digital Isolators," Application Report SLLA198, Texas Instruments (2006).

[107] K. Xu, “Integrated Silicon Directly Modulated Light Source Using p-Well 
in Standard CMOS Technology," IEEE Sens. J., vol. 16, no. 16, pp. 6184 6191, 2016, 10.1109/JSEN.2016.2582840.

[108] K. Xu, "Electro-Optical Modulation Processes in Si-PMOSFET LEDs Operating in the Avalanche Light Emission Mode," IEEE Tran. Electron Devices, vol. 61, no. 6, pp. 2085 - 2092, 2014, 10.1109/TED.2014.2318277.

[109] C. Favi and E. Charbon, "Techniques for fully integrated intra-/interchip optical communication," Proc. 45th ACM/IEEE Design Automation Conf., pp. 343-344, 2008.

[110] M. du Plessis, H. Wen, and E. Bellotti, "Temperature characteristics of hot electron electroluminescence in silicon," Opt. Exp., vol. 23, no. 10, pp. 12605-12612, 2015, 10.1364/OE.23.012605.

[111] K. Xu, "Monolithically integrated Si gate-controlled light-emitting device: science and properties," Jrnl. Opt., vol. 20, no. 2, pp. 024013, 2018, 10.1088/2040-8986/aaa2b7.

[112] L. W. Snyman, H. Aharoni and M. du Plessis, "Characterization of breakdown phenomena in light emitting silicon $\mathrm{n}^{+} \mathrm{p}$ diodes," J. Appl. Phys., vol. 84, no. 5, pp. 2953-2959, 1998, 10.1063/1.368401.

[113] K. Xu, L. Huang, Z. Zhang, J. Zhao, L. W. Snyman and J. W. Swart, "Light emission from a poly-silicon device with carrier injection engineering," Mat. Sci. Eng.: B, vol. 231, pp. 28 - 31, 2018, 10.1016/j.mseb.2018.07.002.

[114] M. du Plessis, H. Aharoni, and L.W. Snyman, "Silicon LEDs fabricated in standard VLSI technology as components for all silicon monolithic integrated optoelectronic systems," IEEE J. Sel. Topics Quantum Electron., vol. 8, no. 6, pp. 1412-1419, 2002.

[115] S. Dutta, R.J.E. Hueting, A.J. Annema, L. Qi, L.K. Nanver and J. Schmitz, "Opto-electronic modeling of light emission from avalanche-mode silicon p+n junctions," J. Appl. Phys., vol. 118, no. 11, 114506, 2015.

[116] S. Dutta, R.J.E. Hueting, V. Agarwal and A.J. Annema, "An integrated optical link in $140 \mathrm{~nm}$ SOI technology," Proc. Conference on Lasers and Electro-Optics (CLEO), Session JW2A, 132, 2016.

[117] A.G. Chynoweth and K.G. McKay, "Photon emission from Avalanche Breakdown in Silicon," Phys. Rev., vol. 102, no. 2, pp. 369-376, 1956.

[118] C. Kurtsiefer, P. Zarda, S. Mayer and H. Weinfurter, “The breakdown flash of Silicon Avalanche Photodiodes - backdoor for eavesdropper attacks?,", J. Mod. Opt., vol. 48, no. 13, pp. 2039-2047, 2001.

[119] Alvin G. Stern, "Design of High Quantum Efficiency and High Resolution, Si/SiGe Avalanche Photodiode Focal Plane Arrays Using Novel, Back Illuminated, Silicon-on-Sapphire Substrates,", in Photodiode-World Activities in 2011, J.W. Park (InTech,2011).

[120] Virtuoso Spectre, Product Version 14.1, Cadence Design Systems, Inc. (Cadence), San Jose, CA 95134, USA (2014).

[121] A. Khanmohammadi, R. Enne, M. Hofbauer, and H. Zimmermann, 
"Monolithic Integrated Optical Random Pulse Generator in High Voltage CMOS Technology,", Proceedings of the European Solid-State Device Research Conference (ESSDERC), pp. 138-141, 2015.

[122] W.J. Kindt, Geiger mode avalanche photodiode arrays, PhD dissertation, Delft University of Technology, The Netherlands (1999).

[123] A. Rochas, Single photon avalanche diodes in CMOS technology, Ph.D. dissertation, École Polytechnique Fédérale de Lausanne, Switzerland, (2003).

[124] M. Sergio and E. Charbon, "An intra-chip electro-optical channel based on CMOS single photon detectors," IEEE International Electron Devices Meeting Tech. Digest, Paper no. 822, pp. 819-822, 2005.

[125] S. Radovanovic, A.J. Annema, and B. Nauta, "A 3 Gb/s optical detector in standard CMOS for $850 \mathrm{~nm}$ optical communication," IEEE J. Solid-State Circuits, vol. 40, no. 8, pp. 1706-1717, 2005.

[126] P. Lombardo, V. Fiore, E. Ragonese and G. Palmisano, "A fullyintegrated half-duplex data/power transfer system with up to $40 \mathrm{Mb} / \mathrm{s}$ data rate, $23 \mathrm{~mW}$ output power and on-chip $5 \mathrm{kV}$ galvanic isolation," IEEE International Solid-State Circuits Conference (ISSCC), pp. 300-301, 2016, 10.1109/ISSCC.2016.7418026.

[127] V. Puliyankot and R. J. E. Hueting, "One-Dimensional Physical Model to Predict the Internal Quantum Efficiency of Si-Based LEDs," IEEE Transactions on Electron Devices, vol. 59, no. 1, pp. 26-34, 2012, 10.1109/TED.2011.2170992.

[128] Roland H. Haitz, "Noise of a Self-Sustaining Avalanche Discharge in Silicon: Low-Frequency Noise Studies," J. Appl. Phys., vol. 38, no. 7, pp. 2935-2946, 1967, 10.1063/1.1710027. 


\section{MULTIPLICATION NOISE IN \\ ON-STATE}

The multiplication noise during avalanche $\left(\sigma_{M}\right)$ can also be estimated from the measured RTSs. Firstly, the transient noise in the On-state of an RTS $\left(I_{n}(t)\right)$ was estimated from the measured $\left(I_{\text {Meas }}(t)\right)$ and the fitted data $\left(I_{R}(t)\right.$, see Eq. (3.2)):

$$
I_{\mathrm{n}}(t)=I_{\text {Meas }}(t)-I_{\mathrm{R}}(t)
$$

Fig. A.1(a) illustrates an example of the procedure of estimating $I_{\mathrm{n}}(t)$ for "decaying" amplitude RTSs. A similar procedure was used for "constant" amplitude RTSs. At a given bias, $I_{n}(t)$ was estimated for each RTS of either type. These $I_{n}(t)^{\prime} s$ were represented as a histogram. An example of the histogram obtained for the "decaying" amplitude RTSs is shown in Fig. A.1(b). A Gaussian $\left(\mathrm{N}\left(b_{\mathrm{n}}, \sigma_{\mathrm{n}}^{2}\right)\right)$ was fitted on this $I_{\mathrm{n}}(t)$ histogram. $\sigma_{\mathrm{n}}$ is the summation of the $\sigma_{\mathrm{M}}$ and the oscilloscope noise $\left(\sigma_{0}\right)$ according to $\sigma_{M}=\sqrt{\sigma_{n}^{2}-\sigma_{0}^{2}} \cdot \sigma_{M}$ is a measure of the fluctuation in the multiplication factor $(M)$ due to the statistical nature of the avalanche process [85].

The estimated $\sigma_{\mathrm{M}}$ for both types of RTSs for all diodes are shown in Fig. A.1(c)-(d). $\sigma_{\mathrm{M}}$ remains almost constant with $V_{\mathrm{EX}}$. During the On-state of an RTS, $M$ is very high [74]. A constant $\sigma_{M}$ implies that the fluctuation in $M$ is relatively constant with $V_{\mathrm{EX}}$. This has been reported earlier that the noise becomes independent of $M$ for large values of $M[85,128]$. Further, noise decreased with increasing avalanche current if the defects would be stable and would not cause any RTS fluctuations [128]. In particular, the unstable defects increase the noise [128]. In our operating bias range, the increasing noise contributed by the unstable defects possibly counteract the decrease in noise induced by the increasing current and therefore a relatively constant $\sigma_{\mathrm{M}}$ is observed. 

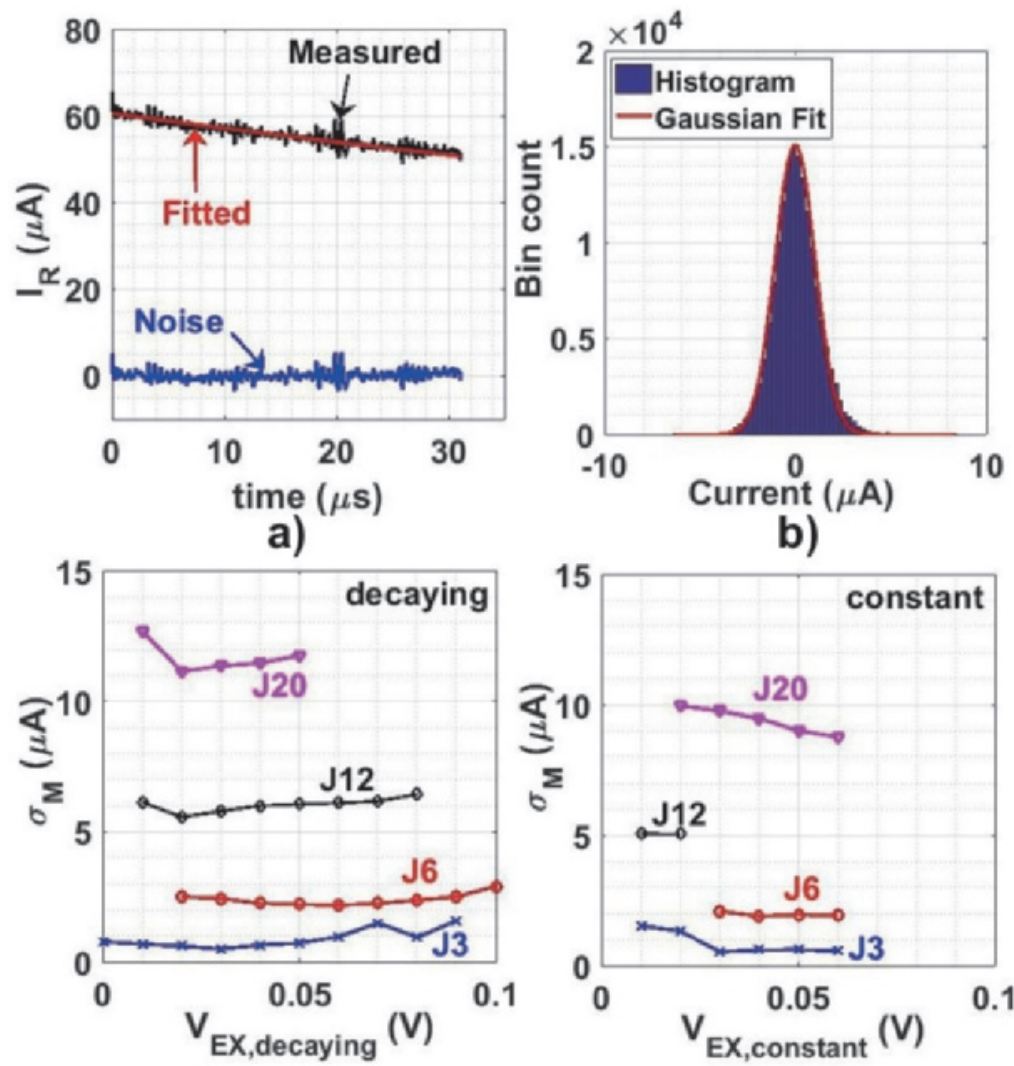

c)

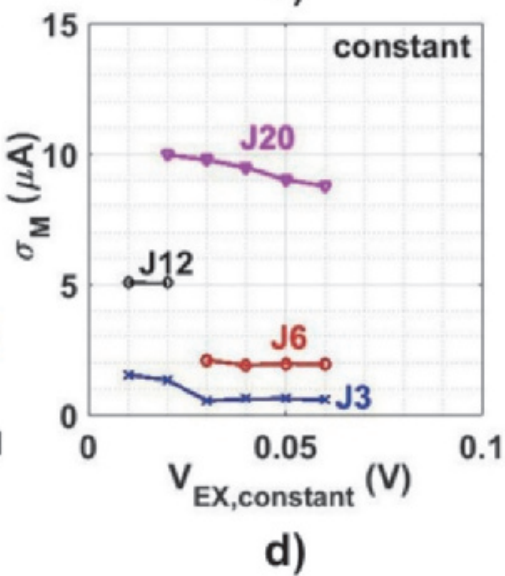

Figure A.1: a) Example procedure for estimating the noise transient $I_{\mathrm{n}}(t)$. b) Example histogram and the fitted Gaussian of the obtained $I_{n}$ of the "decaying" amplitude RTSs in J3 at $V_{\mathrm{R}}=13.87 \mathrm{~V}$. c) $\sigma_{\mathrm{M}}$ for the "decaying" amplitude RTSs. d) $\sigma_{M}$ for the "constant" amplitude RTSs.

In addition, $\sigma_{\mathrm{M}}$ increases for larger diodes. This is because for a larger diode, the number of defects increases, resulting in noise originating from multiple RTS phenomena [97]. 


\section{DISTRIBUTION OF PEAK CURRENT}

A mean $I_{\text {peak }}$ was assumed in section 3.5. As discussed there, in large diodes, the avalanche most likely spreads to nearby defects by lateral transport of free carriers and emission of secondary photons. This effect can be observed from the $I_{\text {peak }}$ histogram. An example of the histogram of the $I_{\text {peak }}$ for "decaying" amplitude RTSs for all diodes at indicated bias settings are shown in Fig. B.1. A wider distribution in $I_{\text {peak }}$ for large diodes is obtained. This is likely due to the random fluctuations in the triggering of avalanche at different defects in larger diodes. 


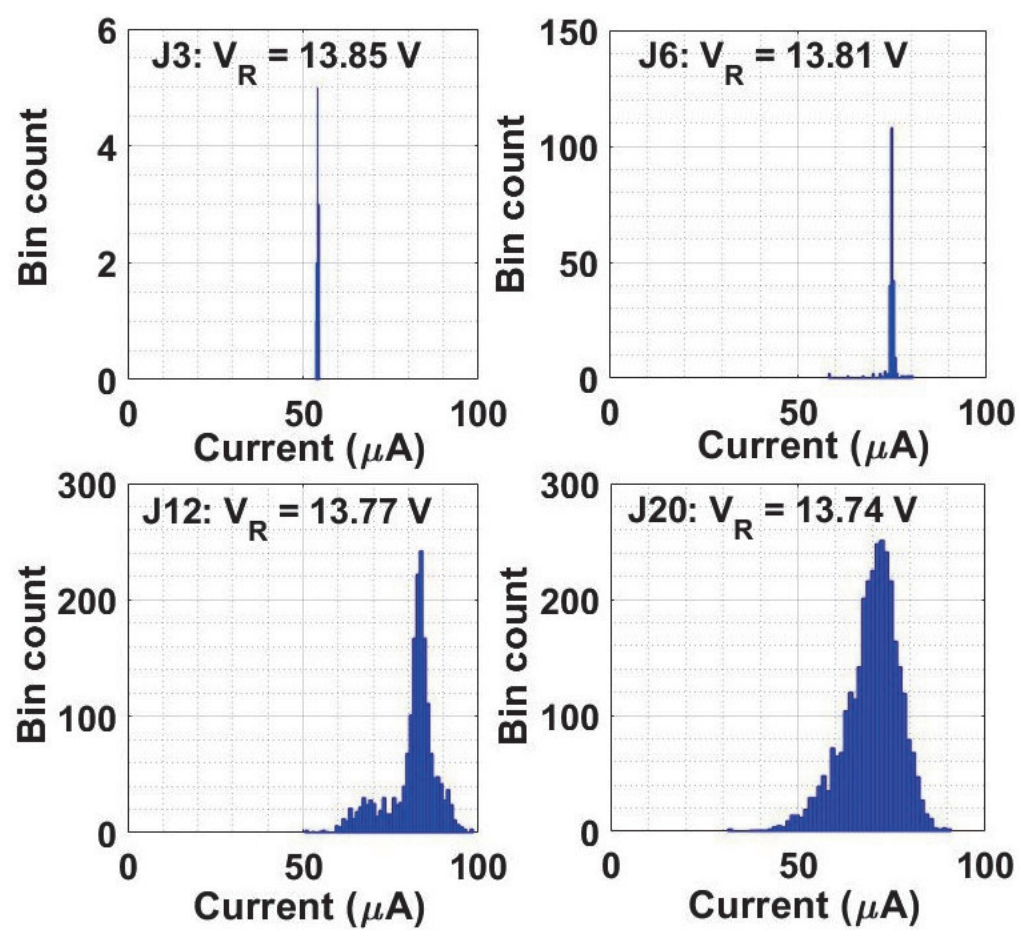

Figure B.1: Histogram of the "decaying" $I_{\text {peak }}$ at specified bias points in all diodes. Y-axis scales are different for clarity. The wider distribution of $I_{\text {peak }}$ in larger diodes can be attributed to random fluctuations at more number of defects. 


\section{APPENDIX}

\section{LIGHT EMISSION PROFILES}

It is commonly known that during avalanche, Si p-n junctions emit visible light $[14,16]$, though with a low internal quantum efficiency $\left(\sim 10^{-5}\right)[71]$. The shallow junctions in these devices allow to observe the light emission from these devices using a visible wavelength camera.

Fig. C.1(a) shows some micrographs of these diodes. Fig. C.1(b) shows the light emission from these devices during the instability region captured using a Nikon D3100 camera with an integration time of $30 \mathrm{~s}$. These emissions were observed at a relatively high $V_{\mathrm{R}}$; at those $V_{\mathrm{R}}$ mostly "constant" amplitude type of RTSs were observed $(F \sim 1)$. At lower $V_{R}$, the light emission intensity was too low to be detected by our camera. Further, for smaller diodes, the light emission appears to be uniform whereas for larger diodes (e.g. J20), the light emitting spots are aligned at the periphery. The light emission from localized spots imply that an RTS in avalanche diodes is a localized phenomenon.

Fig. C.1(c) shows the normalized intensity profiles of the light emission. A non-uniform emission from the localized spots can be seen for the large diodes (e.g. J20). Fig. C.1(d) shows the contour plots of the light emission and localized light emitting spots in large devices can be observed.

From Fig. C.1(b) and the camera resolution, the area of the light emitting spots can be estimated. At the measured camera settings, one image pixel area $\left(A_{\text {pixel }}\right) \sim 0.002 \mu \mathrm{m}^{2}$. From the intensity profiles (Fig. C.1(c)), the number of pixels with an intensity higher than a threshold $\left(N_{\text {pixels }}\right)$ was estimated. Then, the area of the light emitting spot was estimated as $A_{\text {spot }}=N_{\text {pixels }} \times A_{\text {pixel }}$. From $A_{\text {spot }}$, an effective $d$ of the light emitting spot was estimated as $d=\sqrt{4 A_{\text {spot }} / \pi}$; the results are shown in Fig. C.1(e). A close agreement with the estimated $d$ in Fig. 3.14(c) was obtained. The $d$ for J12 is larger than that for J20 as was also estimated in Fig. 3.14(c). The slight difference with the $d$ estimated using the $I_{\text {peak }}-V_{\mathrm{EX}}$ characteristics can be attributed to the low intensity of emission at some pixels that could 
a)
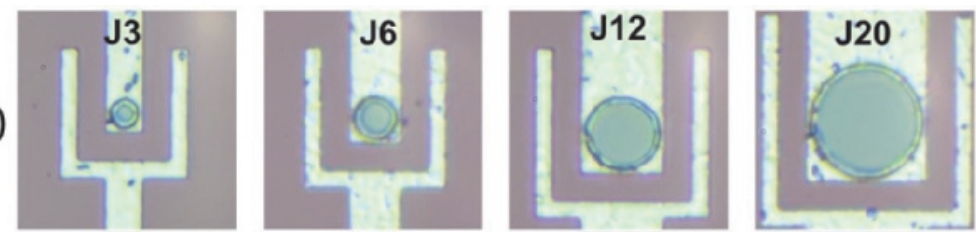

b)
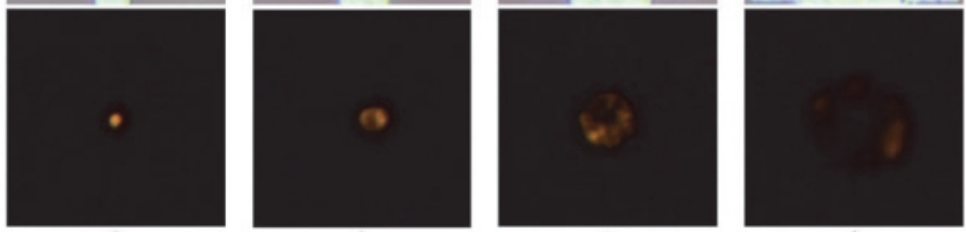

c)
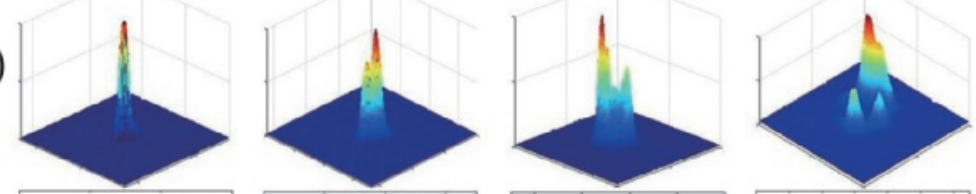

d)
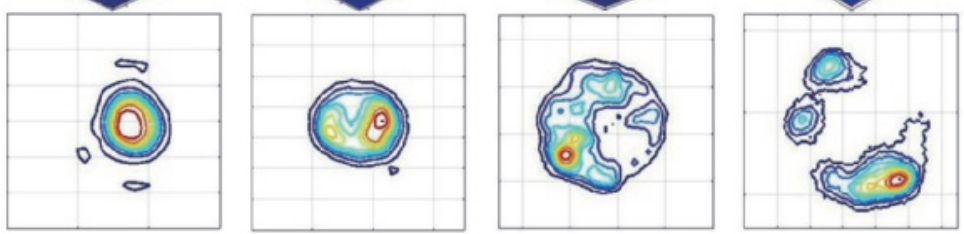

e) \begin{tabular}{|l|l|l|l|}
\hline d $~ 0.72 \mu m$ & d $\sim 1.35 \mu m$ & d $\sim 2.34 \mu m$ & d $\sim 2.06 \mu m$ \\
\hline
\end{tabular}

Figure C.1: a) Device micrographs. b) Light emission captured using a Nikon D3100 Visible wavelength camera with an integration time of 30 s. c) Normalized intensity profiles, d) Normalized contour profiles of the light emission from these junctions. Note that (b) is to scale whereas for (c) and (d) the axis scales are different to enhance the clarity. e) Estimated $d$ of the defects causing "constant" amplitude RTSs from the light emitting spots.

not be measured by the available camera (Nikon D3100). 


\section{LIST OF PUBLICATIONS}

\section{Peer reviewed}

1. V. Agarwal, S. Dutta, A. J. Annema, R. J. E. Hueting, M.-J. Lee, E. Charbon and B. Nauta, "Optocoupling in CMOS", presented at the IEEE Electron Devices Meeting (IEDM) 2018.

2. V. Agarwal, A.J. Annema, R.J.E. Hueting, S. Dutta, Lis K. Nanver, and B. Nauta, “Data transmission capabilities of silicon avalanche diodes", IEEE Transactions on Electron Devices, vol. 65, no. 11, pp. 4883-4890, 2018.

3. R.J.E. Hueting, S. Dutta, V. Agarwal and A.J. Annema, "Figures of merit of avalanchemode silicon LEDs (invited)", $5^{\text {th }}$ Sensors, MEMS and Electro-Optical Systems (SMEOS), 2018.

4. Lis K. Nanver, M. Krakers, V. Agarwal, R.J.E. Hueting, S. Dutta and A.J. Annema, "Investigation of light-emission and avalanche-current mechanisms with PureB SPAD devices (invited)", $5^{\text {th }}$ Sensors, MEMS and Electro-Optical Systems (SMEOS), 2018.

5. V. Agarwal, S. Dutta, A.J. Annema, R.J.E. Hueting, Lis K. Nanver, and B. Nauta, "Random telegraph signal phenomena in ultra-shallow $\mathrm{p}^{+} \mathrm{n}$ silicon avalanche diodes", IEEE Journal of Electron Devices Society, vol. 6, no. 1, pp. 642-652, 2018.

6. V. Agarwal, S. Dutta, A.J. Annema, R.J.E. Hueting, P.G. Steeneken, and B. Nauta, “Low power wide spectrum optical transmitter using avalanche mode LEDs in SOI CMOS technology", Optics Express, vol. 25, no. 15, pp. 16981-16995, 2017.

7. S. Dutta, P.G. Steeneken, V. Agarwal, J. Schmitz, A.J. Annema, and R.J.E. Hueting, "The avalanche-mode superjunction LED", IEEE Transactions on Electron Devices, vol. 64, no. 4, pp. 1612-1618, 2017.

8. S. Dutta, V. Agarwal, R.J.E. Hueting, J. Schmitz, and A.J. Annema, "Monolithic optical link in silicon-on-insulator CMOS technology", Optics Express, vol. 25, no. 5, pp. 5440-5456, 2017.

9. R.J.E. Hueting, A. Heringa, B.K. Boksteen, S. Dutta, A. Ferrara, V. Agarwal and A.J. Annema, "An Improved Analytical Model for Carrier Multiplication Near Breakdown in Diodes", IEEE Transactions on Electron Devices, vol. 64, no. 1, pp. 264-270, 2017.

10. V. Agarwal, A.J. Annema, S. Dutta, R.J.E. Hueting, L.K. Nanver, and B. Nauta, “Random Telegraph Signal phenomena in avalanche mode diodes: Application to SPADs", proceedings of the $46^{\text {th }}$ European Solid-State Device Research Conference (ESSDERC), pp. 264-267, 2016.

11. S. Dutta, R.J. E. Hueting, V. Agarwal, and A.J. Annema, "An integrated optical link in $140 \mathrm{~nm}$ SOI technology", proceedings of the Conference on Lasers and Electro-Optics (CLEO), Session JW2A, p. 132, 2016.

\section{Others}

1. V. Agarwal, S. Dutta, A.J. Annema, R.J.E. Hueting, Lis K. Nanver, and B. Nauta, "Random telegraph signal phenomena in avalanche diodes", ICT Open, Amersfoort, The Netherlands, 2017. 
2. S. Dutta, V. Agarwal, A. J. Annema, R.J.E. Hueting and J. Schmitz "Optocoupling in standard CMOS for smart power (invited)", Emerging Technologies Conference (ETCMOS), Warsaw, vol. 6, Advanced Materials and Optoelectronics Track, pp. 45-61, 2017.

3. Newspaper article: "Avalanche-mode silicon LEDs improve optical coupling in CMOS integrated circuits", LaserFocusWorld, January 2018.

4. Newspaper article: "De optokoppelaar is eindelijk geïntegreerd in cmos", Bits\&Chips, December 2018. 


\section{ACKNOWLEDGEMENTS}

Spending four and a half years in the integrated circuit design group has been a great learning experience for me, both personally and professionally. Many people have helped me in achieving this thesis and I would take this opportunity to thank them here.

First, I would like to thank AJ, Ray and Bram for selecting me after the interviews. Thanks for giving me an opportunity to work on this exciting interdisciplinary project. Thanks to my daily supervisor AJ, who has been a critical in-house reviewer so that our results were very clear and presentable. Due to an extensive in-house reviewing, external reviewers and readers usually found our results very friendly and readable. Also thanks for your patience, enthusiasm and motivation. I would like to thank my other daily supervisor, Ray, who has helped me a lot in the understanding and functionality of device physics. Your constant encouragement was very important in keeping myself motivated throughout PhD. Also, thanks for teaching me many many things about systematically preparing good manuscripts and presentations. Thanks Bram for so many exciting discussions. It was always encouraging to talk to you and you could find a way to inspire me during the conversation. I have been fortunate to have you as my mentor. I would also thank Jurriaan for a lot of interesting discussions regarding device physics and acting as an unbiased reviewer for most of my manuscripts. Your insights were very helpful during the preparation of those manuscripts. Thanks Lis for providing me with very interesting pure boron devices. We have understood many important physics issues due to the studies on these high quality devices.

I would like to thank Myung-Jae Lee and prof. Edoardo Charbon for their insights into the SPAD design. Also, other discussions with them have been very helpful.

Many thanks to Henk and Gerard for their immense support during the design and measurements of the chips. Thanks Gerard for setting up the CAD environment and helping me to understand more about this CMOS technology and PCB design. Thanks Henk for discussing number of measurement plans and also providing so many urgent help. Also, thanks to the Sander from the IDS group for providing additional help to familiarize myself with the device characterization. Thanks Gerdien for managing so many things so efficiently. From the day I was selected for the ICD group to this day, you have provided a constant and timely help to keep things on track.

I would also thank my project partner Satadal for a very nice experience throughout this project. Long interesting discussions and working together was always fun. We have tried so many ideas together. Although many of them were too "fancy", each of the experiments was always fun and it was more fun to understand the results.

I am also grateful to user-committee members of this project for attending and providing constructive feedback on our work. I would like to thank Dr. Peter Steeneken, Dr. Dimitar Dochev and Maarten Swanenberg for the finishing of chips and clarifying many doubts about the technology. Also thanks to Gerard Voshaar for many interesting discussions. Thanks Herma for organizing the user committee meetings and assisting in $\mathrm{OiC}$ project. Thanks to NXP semiconductors for silicon contribution.

I would like to thank my PhD roommates Maurits, Vijay, Sajad, Claudia, Zhiliang and Morteza. It was always fun to work with you guys and spend many game nights. The international working atmosphere was a delight for me; I learnt something about many different cultures now. I would also thank other ICD members: Eric, Ronan, Mark, Anoop, Saifulla, Harijot, Alexander, Ali, Maikel, Ines, Andreas, Harish, Joeri, YC, Chris, Labrinus, Bart, Joep, DJ, Reda, Daniel, Hugo and Johan for their feedback in design reviews, measurements 
and presentations. Also thanks to Gaurav, Sourish and Xingu from the IDS group.

I would like to mention my family. My parents, sisters, brother and uncle have always encouraged and supported me along the way. I have been lucky to have a loving and supporting family. I want to thank Ankit and Bhowmick for being good friends always. Thanks Himanshu (my roommate in Bangalore) for helping me to make my decision regarding pursuing a PhD. Also thanks for all your wisdom during dinners, I must say that I have missed it. Thanks to Vaibhav for encouraging me to always believe in myself. I would also thank my Indian friends in the Netherlands: Devashish, Manas, Vishal, Pramod, Vishakha, Himanshu, Nachiket, Ketakee, Naveen, Poorvi, Shantanu, Praneeth and Akshita for potluck dinners and interesting discussions. Those experiences never let me feel homesick.

Finally, special mention of my wife Bina. You have tolerated many many of my long working days (and nights). Your immense support and understanding has been invaluable in me completing this thesis. 



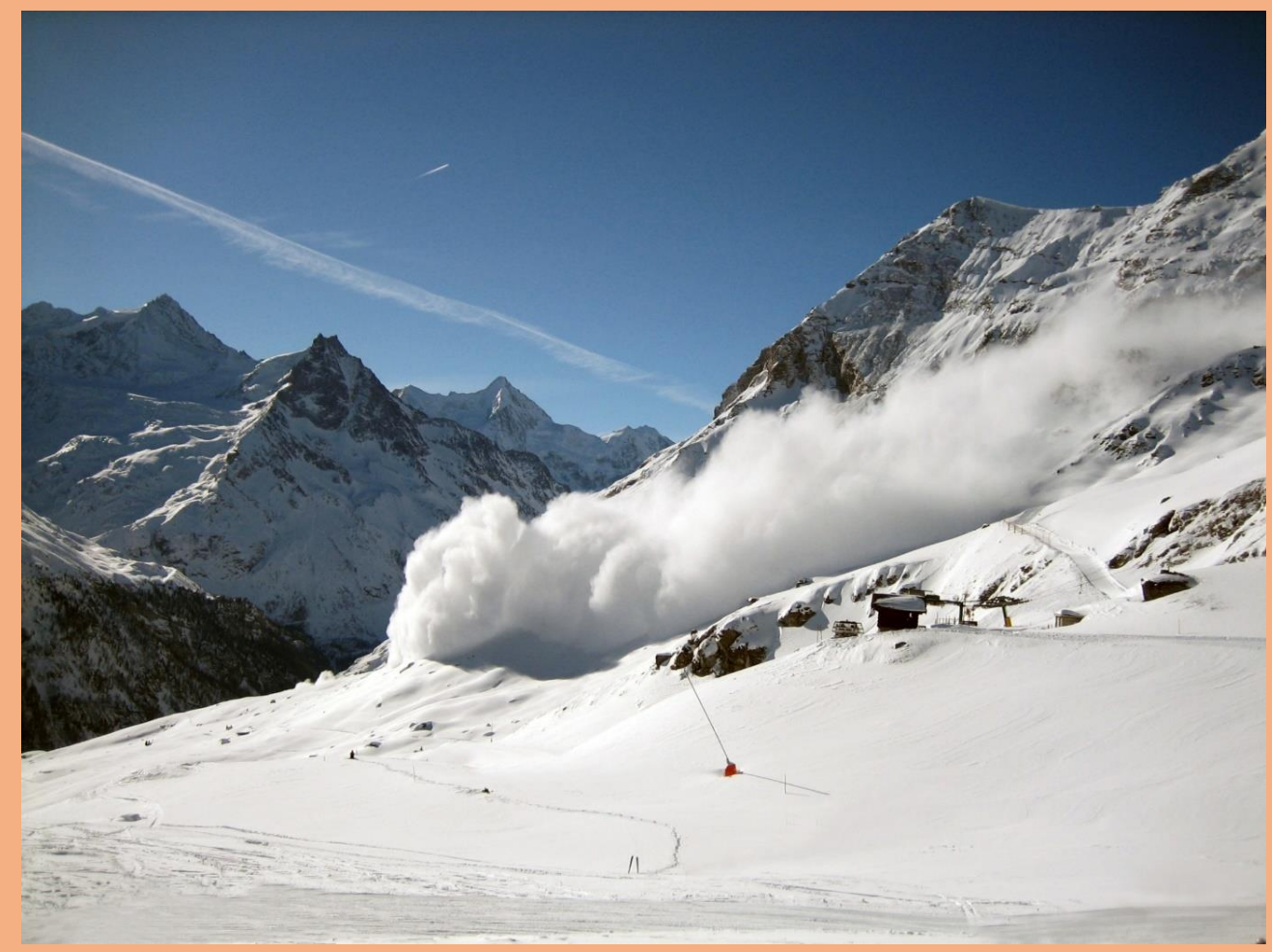

